\title{
Development of Control Models and a Robust Multivariable Controller for Surface Shape Control
}

\author{
S. E. Winters
}

U.S. Department of Energy

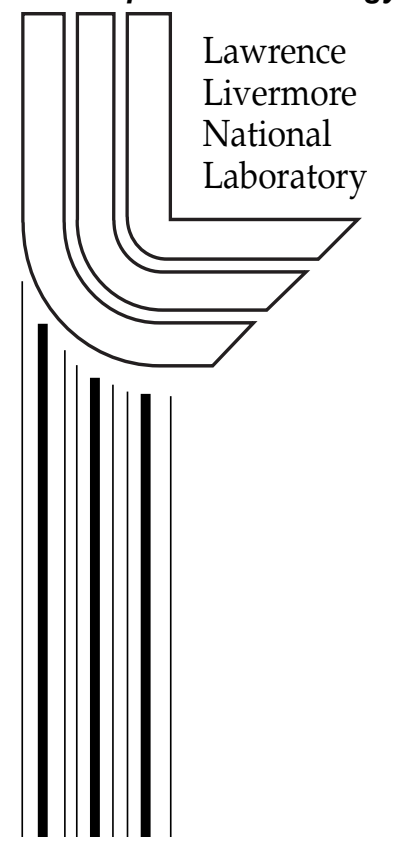

June 18, 2003 
This document was prepared as an account of work sponsored by an agency of the United States Government. Neither the United States Government nor the University of California nor any of their employees, makes any warranty, express or implied, or assumes any legal liability or responsibility for the accuracy, completeness, or usefulness of any information, apparatus, product, or process disclosed, or represents that its use would not infringe privately owned rights. Reference herein to any specific commercial product, process, or service by trade name, trademark, manufacturer, or otherwise, does not necessarily constitute or imply its endorsement, recommendation, or favoring by the United States Government or the University of California. The views and opinions of authors expressed herein do not necessarily state or reflect those of the United States Government or the University of California, and shall not be used for advertising or product endorsement purposes.

This work was performed under the auspices of the U.S. Department of Energy by University of California, Lawrence Livermore National Laboratory under Contract W7405-Eng-48. 


\title{
DEVELOPMENT OF CONTROL MODELS
}

\section{AND A ROBUST MULTIVARIABLE CONTROLLER \\ FOR SURFACE SHAPE CONTROL}

\author{
By
}

SCOTT ERIC WINTERS

B.S. (University of California, Davis) 1991

M.S. (University of California, Davis) 1992

\section{DISSERTATION}

Submitted in partial satisfaction of the requirements for the degree of

DOCTOR OF PHILOSOPHY

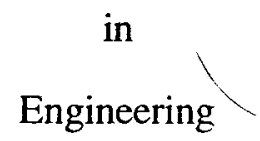

in the

OFFICE OF GRADUATE STUDIES

of the

UNIVERSITY OF CALIFORNIA

DAVIS

Approved:

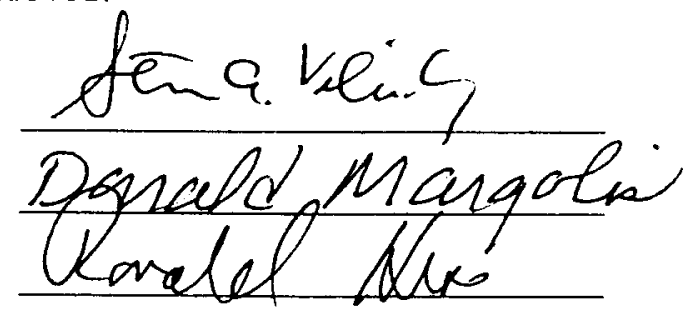

Committee in charge 


\begin{abstract}
Surface shape control techniques are applied to many diverse disciplines, such as adaptive optics, noise control, aircraft flutter control and satellites, with an objective to achieve a desirable shape for an elastic body by the application of distributed control forces. Achieving the desirable shape is influenced by many factors, such as, actuator locations, sensor locations, surface precision and controller performance. Building prototypes to complete design optimizations or controller development can be costly or impractical. This shortfall, puts significant value in developing accurate modeling and control simulation approaches. This thesis will focus on the field of adaptive optics, although these developments have the potential for application in many other fields.

A static finite element model is developed and validated using a large aperture interferometer system. This model is then integrated into a control model using a linear least squares algorithm and Shack-Hartmann sensor. The model is successfully exercised showing functionality for various wavefront aberrations. Utilizing a verified model shows significant value in simulating static surface shape control problems with quantifiable uncertainties.

A new dynamic model for a seven actuator deformable mirror is presented and its accuracy is proven through experiment. Bond graph techniques are used to generate the state space model of the multi-actuator deformable mirror including piezo-electric actuator dynamics. Using this verified model, a robust multi-input multi-output (MIMO) $\mathrm{H}_{\infty}$ controller is designed and implemented. This controller proved superior performance as compared to a standard proportional-integral controller (PI) design.
\end{abstract}


This dissertation is dedicated in loving memory of my mom, Myrna M. Winters. 


\section{FORWARD}

I wish first and foremost to thank my wonderful wife, for it was she who always seemed to fall victim of my schedule. I love you very much and thank you for understanding. I wish also to thank my entire family, since I could have never realistically completed this document without their love and support.

I would like to thank my advisor, Dr. Steven A. Velinsky for his advice and guidance over the past six years. His friendship has contributed to my personal growth and made the difficult times much easier. Additionally, I would like to thank Dr. Chung for his contributions to the control sections of this work. His efforts greatly contributed to this research. The members of my dissertation committee, Dr. Hess and Dr. Margolis, deserve recognition for their contributions.

Lastly, I would like to thank the many people at Lawrence Livermore National Laboratory who contributed to this research and greatly contributed to my knowledge. 


\section{ACKNOWLEDGMENTS}

This work was partially performed under the auspices of the U.S. Department of

Energy by the Lawrence Livermore National Laboratory under contract W-7405Eng-48.

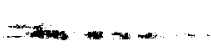




\section{TABLE OF CONTENTS}

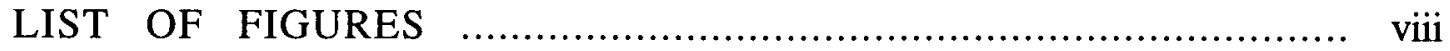

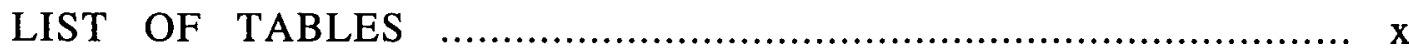

CHAPTER 1 - INTRODUCTION

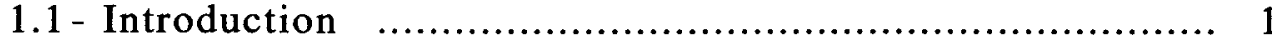

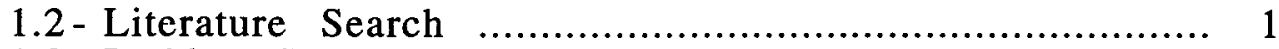

1.3 - Problem Statement and Objective ................................. 5

CHAPTER 2 - OVERVIEW OF ADAPTIVE OPTICS SYSTEMS

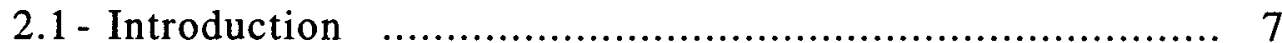

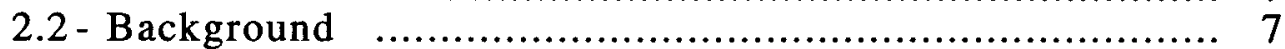

2.3 - National Ignition Facility Adaptive Optics System .................. 9

2.4 - Deformable Mirror Configuration .................................... 10

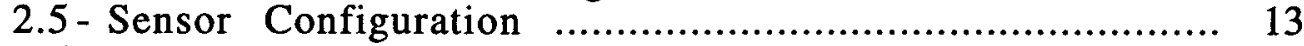

2.6 - Controller Configuration ............................................ 14

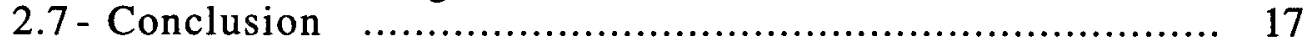

CHAPTER 3 - STATIC SURFACE SHAPE MODELING

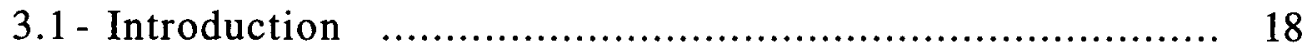

3.2 - Finite Element Modeling ......................................... 18

3.3 - Experimental Tests ............................................. 23

3.4-Model Verification ................................................. 26

3.5 - Conclusion .................................................. 32

CHAPTER 4 - STATIC (GEOMETRICAL) SURFACE SHAPE CONTROL

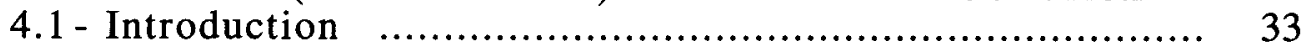

4.2 - Control System Description ......................................... 33

4.3 - Plant Model .................................................... 34

4.4 - Sensor Model .................................................... 35

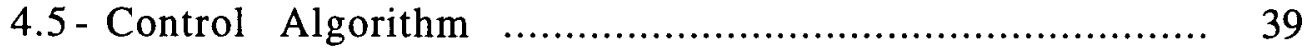

4.6- Control Simulation ................................................. 39

4.7- Performance Comparison ............................................. 44

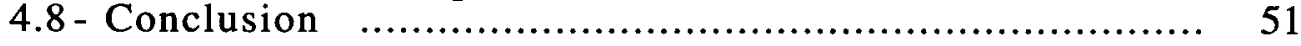

CHAPTER 5 - DYNAMIC SURFACE SHAPE MODELING

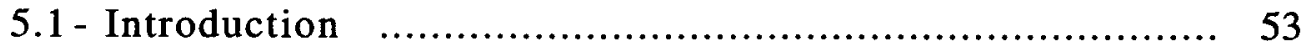

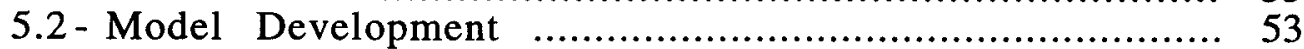

5.3 - Model Reduction ............................................... 55

5.4 - Derivation of Dynamical Equations of Motion ...................... 59

5.5 - Experimental Verification ............................................ 68

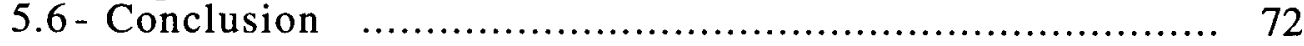

CHAPTER 6 - DYNAMIC SURFACE SHAPE CONTROL

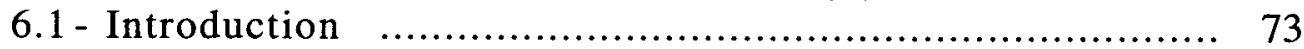

6.2 - Control Approach and Related Research …............................ 73

6.3 - Control Model ........................................................ 74

6.4 - Design Objectives ............................................. 75

6.5 - SISO Control Simulations ..................................... 78

6.6 - MIMO Proportional Integral Control Simulations ................... 86 
6.7 - MIMO $\mathrm{H}_{\infty}$ Control Simulations ................................. 96

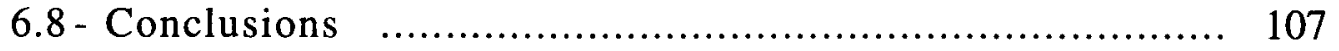

CHAPTER 7 - CONCLUSIONS AND RECOMMENDATIONS

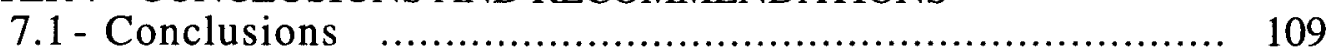

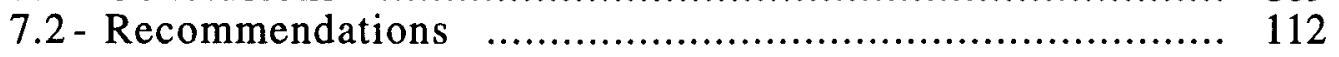

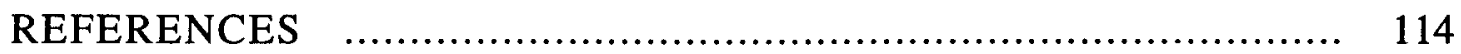

APPENDIX A - STATIC SURFACE SHAPE CONTROL CODE ................ 119

APPENDIX B - C-FIELD FOR A PIEZOELECTRIC ACTUATOR ............. 128

APPENDIX C - DERIVATION OF EQUATIONS OF MOTION ................. 130

APPENDIX D - SINGLE ACTUATOR MODEL CODE ....................... 133

APPENDIX E - MULTI-ACTUATOR MODEL CODE .......................... 136 


\section{LIST OF FIGURES}

Figure 2.1 - Single chain of NIF showing adaptive optic components .......... 10

Figure 2.2 - Typical segmented and continuos active mirrors .................... 11

Figure 2.3 - Different piezoelectric mirrors ….................................... 12

Figure 2.4 - NIF prototype deformable mirror ................................ 13

Figure 2.5 - Hartmann wavefront sensor technique .......................... 15

Figure 2.6 - Typical output from Hartmann sensor ........................... 16

Figure 2.7 - Controller configuration for the NIF adaptive optics system ....... 16

Figure 3.1 - National Ignition Facility prototype deformable mirror ............. 19

Figure 3.2 - Finite element model of deformable mirror ......................... 21

Figure 3.3 - Schematic of deformable mirror including actuators

and clear aperture ............................................. 22

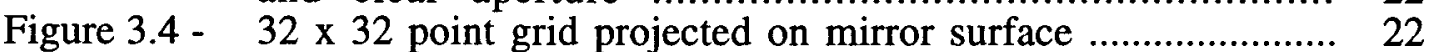

Figure 3.5 - Influence function for actuator $\# 20 \ldots \ldots \ldots \ldots \ldots \ldots \ldots \ldots \ldots \ldots . . . \ldots \ldots \ldots$

Figure 3.6 - Interferometer schematic layout and picture ....................... 24

Figure 3.7 - Experimental data for actuator \#20 ................................ 26

Figure 3.8 - Difference between model and experimental data for

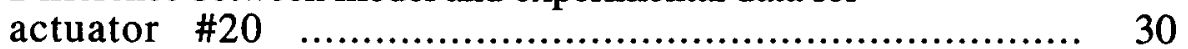

Figure 3.9 - Line plot for model and experiment for actuator \#20 (y-dir.) ...... 31

Figure 3.10 - Line plot for model and experiment for actuator \#20 (x-dir.) ...... 31

Figure 4.1 - General MIMO block diagram .................................. 34

Figure 4.2 - Schematic of NIF deformable mirror w/ actuators

and projected lenslets......................................... 36

Figure 4.3 - Coordinates and base points used in finite difference equations ... 38

Figure 4.4 - $\quad$ Typical 2nd order aberration ...................................... 41

Figure 4.5 - $\quad$ Residual after correction of 2 nd order aberration (full scale) ....... 41

Figure 4.6 - Same as Figure 4.5 with different scâle ............................. 42

Figure 4.7 - Typical 4th order aberration ........................................ 42

Figure 4.8 - $\quad$ Residual after correction of 4th order aberration (full scale) ....... 43

Figure 4.9 - $\quad$ Same as Figure 4.8 with different scale …....................... 43

Figure 4.10 - Normalized Gaussian influence function using Equation (4.14) ... 46

Figure 4.11 - Difference between Gaussian and experimental results ............ 46

Figure 4.12 - Line plot of Gaussian model and experiment for act. \#20 (y-dir.) . 47

Figure 4.13 - Line plot of Gaussian model and experiment for act. \#20-(x-dir.) . 47

Figure 4.14 - Correction of piston aberration with Gaussian model .............. 48

Figure 4.15 - Correction of piston aberration with FE model ................... 48

Figure 4.16 - Same as Fig. 4.15 with different scale ......................... 49

Figure 4.17 - Residual after correction of 2nd order using Gaussian model ...... 49

Figure 4.18 - Same as Figure 4.17 with different scale ........................... 50

Figure 4.19 - Residual after correction of 4th order aberration with Gaussian model ................................................. 50

Figure 4.20 - Same as Fig. 4.19 with different scale ............................... 51

Figure 5.1 - Plate and actuator layout ......................................... 54

Figure 5.2 - Modal density plot for actuator plate system ....................... 59

Figure 5.3 - Simplified actuator and plate model ............................... 62

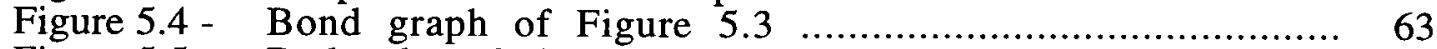

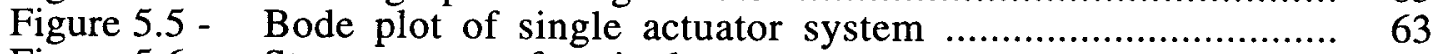

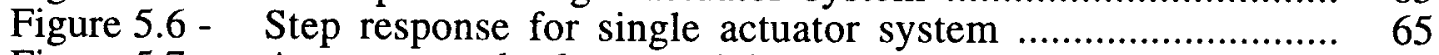

Figure 5.7 - Actuator and plate model .......................................... 65

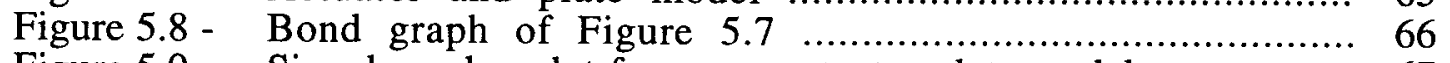

Figure 5.9 - Singular value plot for seven actuator plate model ...................... 67 
Figure 5.10 - Step response for seven actuator model with input

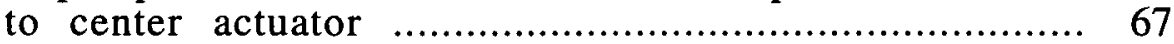

Figure 5.11- Block diagram of experimental setup ................................. 69

Figure 5.12 - Experimental setup for frequency response test .................... 70

Figure 5.13 - Detail of experimental setup .......................................... 71

Figure 5.14 - Experimental frequency response data .............................. 71

Figure 5.15 - Frequency response plot from Marlow (1994) …….............. $\quad 72$

Figure 6.1 - General MIMO block diagram ……............................... 75

Figure 6.2 - Step response $(\mathrm{y} / \mathrm{r})$ of ideal system to be matched ................... 80

Figure 6.3 - Step response (y/d) of ideal system to be matched ................... 81

Figure 6.4 - Open loop (L) Bode plot of system to be matched .................... 83

Figure 6.5 - Bode plot of matching system complimentary sensitivity (T) function ........................................... 83

Figure 6.6 - Bode plot of matching system sensitivity (S) function ……......... 84

Figure 6.7 - Step response for open loop, closed loop and matching system .... 84

Figure 6.8 - Bode plot of complimentary sensitivity (T) for closed loop system …............................................ 85

Figure 6.9 - Bode plot of sensitivity (S) for closed loop system ................... 85

Figure 6.10 - Bode plot of return ratio (L) for closed loop system ................. 86

Figure 6.11 - Open loop singular value plot ……................................ 89

Figure 6.12 - Open loop step response ……................................... 90

Figure 6.13 - Frequency plot of return ratio (L) using PI control ................ 90

Figure 6.14 - Frequency plot of complimentary sensitivity (T) using PI control . 91

Figure 6.15 - Frequency plot of sensitivity (S) using PI control ................... 91

Figure 6.16 - Closed loop step response using PI controller ....................... 92

Figure 6.17 - Open loop singular value plot of modified plant model ............... 94

Figure 6.18 - Open loop step response of modified plant model .................... 94

Figure 6.19 - Frequency response of (L) using PI controller ...................... 95

Figure 6.20 - Frequency response of complimentary sensitivity (T) using PI controller …............................................... 96

Figure 6.21 - Frequency response of sensitivity (S) using PI controller ........... 95

Figure 6.22 - Step response using PI controller .................................... 96

Figure 6.23 - Augmented plant and controller for $\mathrm{H}_{\infty}$ design ...................... 98

Figure 6.24 - Return ratio (L) frequency plot for $\mathrm{H}_{\infty}$ design ....................... 102

Figure 6.25 - Frequency plot of complimentary sensitivity (T) for $\mathrm{H}_{\infty}$ design .... 103

Figure 6.26 - Frequency plot of sensitivity (S) for $\mathrm{H}_{\infty}$ design .................... 103

Figure 6.27 - Step response applied to the center actuator ........................... 104

Figure 6.28 - Return ratio (L) frequency plot for $\mathrm{H}_{\infty}$ design ........................ 105

Figure 6.29 - Frequency plot of complimentary sensitivity (T) for $\mathrm{H}_{\infty}$ design .... 105

Figure 6.30 - Frequency plot of sensitivity (S) using $\mathrm{H}_{\infty}$ controller .............. 106

Figure 6.31 - Step response using $\mathrm{H}_{\infty}$ controller .................................. 107 


\section{LIST OF TABLES}

Table 3.1 - $\quad$ Summary of interferometer characterization ....................... 26

Table 3.2 - $\quad$ Percent difference between model and experimental data ........... 29

Table 4.1 - Comparison between Gaussian and finite element plant models ... 45

Table 6.1 - $\quad$ Performance comparison of PI and $\mathrm{H}_{\infty}$ controller designs .......... 102

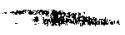




\section{CHAPTER 1 - INTRODUCTION}

\subsection{Introduction}

Surface shape control techniques are applied to many diverse disciplines, such as adaptive optics, noise control, aircraft flutter control and satellites, with an objective to achieve a desirable shape for an elastic body by the application of distributed control forces. Depending on the system, many factors contribute to the ability to achieve the desirable shape, such as actuator locations, sensor locations, surface precision and controller characteristics. For example, in adaptive optics systems, mirror surfaces are precision machined and polished to nanometer tolerances and assembly techniques are utilized to reduce errors to a corresponding level. Accordingly, controllers must be designed to maintain a similar level of precision to achieve accurate surface shape control in the presence of disturbances.

Although surface shape control is applied to diverse fields, this application will focus on adaptive optics systems. However, the general nature of this work will allow it the potential for application in many other fields. As such, the purpose of this thesis is to develop validated static and dynamic models for control purposes, and then to investigate the use of a modern multi-input multi-output control approach for dynamic surface shape control.

\subsection{Literature Search}

Over the past four decades, surface shape control techniques have been applied to many diverse technical areas. These include, but are not limited to, adaptive optics (Creedon and Lindgren, 1970; Apollonov, et al, 1990; Chiarappa and Claysmith, 1981; Ealey, 1991), noise control for marine vessels and aircraft fuselage panels (Dimitriadis and Fuller, 1989; Walker and Yaneskek, 1976a), aircraft flutter control (Livne and Wei-Lin, 1995) and space technology such as satellite antennas (Junkins, 1990). The object of 
surface shape control is to achieve a desirable shape for a given elastic body by the application of distributed control forces and/or displacements. Due to the diverse technical areas in which surface shape control is utilized, an extremely large body of literature exists in various fields. Fortunately, three literature review summaries were recently published. Junkins (1990) edited the book Mechanics and Control of Large Flexible Structures, which included an update of a literature review completed in 1984 and work in the area of shape control. The majority of this work focused on space related structures. Another comprehensive literature review was completed by Meirovitch (1990). In his review, Meirovitch covered many topics related to modeling and control of structures for a variety of applications. Lastly, Tyson (1998) reviews the concept of shape control related to adaptive optics in his book Principles of Adaptive Optics. Despite the large body of existing literature, two areas have received relatively little attention. Those being the issue of actuator and sensor dynamics and their role in the overall system performance (Meirovitch, 1990), and the issue of dynamic -actuator coupling and its effect on controllability. A deep understanding of both veshese issues is essential for adaptive optic systems to meet future more stringent requirements.

Dynamic modeling has been researched for both SISO and MIMO systems. Related to adaptive optics, Marlow (1994) developed the electro-mechanical equations governing the motion of the surface of a deformable mirror for a single actuator. Wirth and Jankevics (1993) use two approaches to model a multi-actuator deformable mirror. First, they assume the deformable mirror is represented by a nondiagonal matrix and ignore high frequency dynamics. The second approach is an experimental technique using the extended Kalman filter to estimate the state variables. Creedon and Lindgren (1970) develop the dynamic equations of a deformable mirror using a modal expansion technique. This approach neglected any actuator dynamics. Livne and Wei-Lin (1994) utilize rational function approximations to develop a linear time invariant state space model of aeroservoelastic systems. This model was used to examine wing/control surface shape 
optimizations. Related specifically to modeling of combined dynamical systems, that is systems comprised of both distributed mass components and point masses, many different approaches are available for use, each having their own advantages and disadvantages. These methods include, but are not limited to, finite elements (Vincent, et al, 1990), finite difference, and classical modal techniques (Margolis, 1976; Nicholson and Bergman, 1986). For dynamic systems, the normal mode approach has an advantage over both finite difference and finite element models by requiring relatively few equations, which helps significantly when developing models for control simulation. Margolis (1980) showed that the normal mode approach model required 1/10 the number of equations as a finite difference model while producing indistinguishable results. However, one disadvantage of the normal mode approach is that no analytical solution exists for many complex models, such as plates.

Concerning static model development, authors have considered both numerical models, such as finite elements (Furber, et at, 1994a;-Bigelow, et al, 1994), and analytical models, such as Gaussian functions (Ealey; functions (Apollonov, et al, 1990). Dimitriadas and Fuller (1990) utilize analytical techniques to solve for the static shape of a circular plate with edge bending moments for controlling sound transmission. When exact solutions do not exist, the errors associated with using most analytical functions are related to the boundary conditions and the so called pinning error effect (Hiddleston, et al, 1991). Numerical approaches, such as the finite element method, do not have these deficiencies since they maintain continuity in displacement, slope and higher derivatives between elements. Therefore, the finite element method seems to be the preferred approach due to its accuracy, flexibility and the availability of commercial software:

Research related to the development of control approaches vary significantly and can be split between static and dynamic control. Static control approaches have been applied to many diverse fields (Meirovitch, 1990), and therefore this review will emphasize 
static surface shape control. Static shape control has an objective to achieve a desirable shape of an elastic body to minimize some criteria. Salmon, et al, (1993), Furber, et al, (1994a) and Tyson (1998) utilized a least squares surface shape control algorithm to minimize the wavefront error in an optical system. Dimitriadis and Fuller (1990) developed a cost function based on the integral of the squared pressure amplitude to minimize sound transmission from a plate. Other authors have researched surface shape optimizations, such as actuator and sensor locations (Shishakov and Shmal'gauzen, 1992; Bushnell, 1979) to minimize a surface fitting error. Lyakhov (1994) and Creedon, et al, (1970) discuss issues related to the number and placement of actuators based on geometric considerations.

There are a significant number of dynamic control approaches for SISO and MIMO systems. In general, these control approaches are for any feedback control system, not just surface shape control. When considering a control approach, stability, performance and robustness must be evaluated (Mayne, 1996). SISO examples include but are not limited

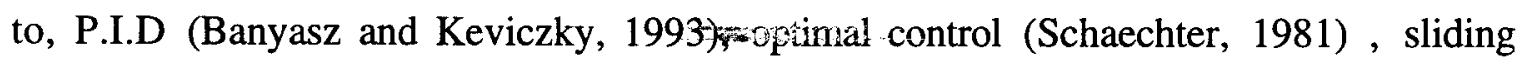
mode control (Slotine and Li, 1991) and adaptive control (Astrom, 1983). The classical P.I.D. control algorithm gives good physical insight, however lacks robustness characteristics found in more modern control approaches (Meirovitch, 1990). Schaechter (1981) presents an optimal local control algorithm for flexible structures. This work uses local state information to synthesize required forces which are proportional to curvature and curvature rate. No information is provided about robustness. Slotine and Li (1991) presented sliding mode control which guarantees a feedback controller which is robust to the uncertainty of the model. Lastly, Astrom (1983) overviews adaptive control with emphasis on model reference adaptive systems and self-tuning regulators. Some popular adaptive techniques are gain scheduling, model reference and self-tuning regulators.

Balas and Doyle (1990), Hyde and Seering (1991), Blanton and Sadek (1992), Fanson, et al (1990), Schaechter (1981), Vincent, et al, (1990) and Silverberg and Weaver 
(1996) have investigated various control problems related to flexible structure or plates. Vincent, et al, (1990) developed a controller to have a flexible plate mimic a rigid one. This work focused on sensor and actuator placement based on controllability and observability. Blanton and Sadek (1990) developed an optimal controller to actively dampen undesirable transient vibrations of a rectangular plate by minimizing the total energy. Lastly, Balas and Doyle (1990) discussed designing linear control algorithms for flexible structures with emphasis on physical system uncertainty. Furthermore, robustness and performance tradeoffs are discussed relative to system model uncertainties.

Fewer approaches have been developed and utilized for MIMO systems. Examples include but are not limited to, $\mathrm{H}_{\infty}$ synthesis (Safonov, et al, 1990), Q-factorization (Sueur and Dauphintanguy, 1991) and Youla synthesis (Brewer, 1995). Although the controller synthesis approaches vary significantly, in general they attempt to accomplish the same goals (e.g., robustness, disturbance rejection, tracking, stability). Safonov, et al, (1990) presented a $\mathrm{H}_{\infty}$ robust controller synthesis for a large space structure and showed its robustness to model uncertainty. Brewer (1995) presented the Youla synthesis technique which allows the direct evaluation of actuator saturation or required effort for input noise and disturbances.

\subsection{Problem Statement and Objective}

A more focused approach on static and dynamic modeling and control of deformable mirrors has the potential to allow the development of higher precision systems and to enable deformable mirrors to meet the anticipated more stringent requirements of the near future. Current published modeling approaches focus on static design parameters which have little use in control simulation (Ealey and Wellman, 1991) or lack reasonable physical correlation to the actual system for design purposes (Gully, et al, 1994). Furthermore, use of validated static and dynamic models for precision surface shape 
control and optimization is lacking. Concerning the more stringent requirements, as sensor technology improves, operating closed-loop bandwidths on the order of tens of kilohertz are expected. This can only be accomplished with the ability to control deformable mirror open loop response to better precision.

This research can be split into two distinct areas: 1) development of a static finite element model, model verification, and implementation of a static controller and 2) development of a dynamic model, model verification, and implementation of a modern dynamic controller. Accordingly, this thesis is organized as follows. Chapter 2 overviews adaptive optics systems utilizing the National Ignition Facility (NIF) adaptive optic system as a specific example. The three major adaptive optic components discussed are the deformable mirror, sensor and electronic controller. Chapter 3 covers the development and validation of a static model. The finite element method is used to develop the model and an interferometric facility at Lawrence Livermore National Laboratory is used to acquired data on the NIF prototype deformable mirror for model validation. In Chapter 4, a static control approach is implemented using the validited model from Chapter 3. The control model is based on a linear least squares approach and the sensor is based on a Shack-Hartmann (Tyson, 1998). Using the control model, typical aberrations are corrected to show model performance and functionality. In Chapter 5, dynamic models are developed of the NIF prototype deformable mirror. Bond graph techniques are utilized to develop the dynamic equations of motion and Raytheon Optical Systems, Incorporated mechanical measurement facility is utilized to acquired data for model validation. In Chapter 6 , single-input singleoutput (SISO) and multi-input multi-output (MIMO) controllers are developed and implemented utilizing the validated models from Chapter 5. Standard proportional-integral (PI) and modern $\mathrm{H}_{\infty}$ controller designs are implemented and results compared. Lastly, Chapter 7 includes a summary and recommendations are made for continued research. 


\section{CHAPTER 2 - OVERVIEW OF ADAPTIVE OPTICS SYSTEMS}

\subsection{Introduction}

The purpose of this chapter is to introduce the field of adaptive optics, which will include the deformable mirror, sensor and controller. For a specific example, the National Ignition Facility (NIF) adaptive optics system will be used for reference. The first section will cover the background of adaptive optics. Next, the National Ignition Facility adaptive optics system will be overviewed. The third section will cover various deformable mirrors, including the NIF prototype deformable mirror. Lastly, controller and sensor configurations will be discussed.

\subsection{Background}

The optics industry, since the development of the laser in the 1950's, has strived to achieve optical components and systems with near diffraction limited performance. As the laser improved in terms of power año peam quality, time-varying distortions induced by laser gain medium and non-linearities, thermal blooming and atmospheric turbulence impeded system performance. Due to these problems, a new generation of adaptive optics were developed, which included the development of the deformable mirror. The deformable mirror is an early application of surface shape control. In general, a deformable mirror is comprised of a planar facesheet with actuators attached to the back surface to provide transverse surface displacements. The first continuous facesheet deformable mirror was developed by Perkin Elmer Corporation in 1970 for NASA. Following this original development, several companies (e.g., Hughes, United Technologies, Rockwell, Lockheed, Itek) developed their own deformable mirrors for both high energy laser systems and atmospheric compensation throughout the 1970's (Ealey, 1991). The 1980's saw defense funding push the specialized development and performance of deformable mirrors to a new level. Some of the leaders in this technology were Itek and United 
Technologies Optical Systems. The late 1980's and 1990's has seen a continued interest in the development of a variety of deformable mirrors for high energy laser systems, such as the National Ignition Facility, and astronomical applications, such as the Keck observatory.

Although, as evident by the above discussion, much work has been completed in the development of deformable mirrors, there are still some areas which are relatively unexplored, lack significant analysis, or lack experimental verification. Two areas which fall in this category are system modeling and control simulation. This lack of analysis can be attributed to the complexity of these systems and general satisfaction with current system's performance. From a static correction approach, the lack of experimentally validated models increases the risk and cost of developing optimized designs. This typically occurs due to the extraordinary cost of equipment needed for experiments. From a dynamic correction approach, bandwidth requirements for most deformable mirrors are under the mechanical resonant frequencies, or controller/sensor limitations restrict bandwidths from reaching deformäblè mirror mechanical resonances. However, as discussed by Lillard (1994), mechameat resonances have shown to be the performance limiting factor. Furthermore, as controller and sensor technologies progress, deformable mirror dynamic characteristics will become increasingly important. The complexity of the deformable mirror system has also limited the activity in the area of modeling and control simulation. The continuous facesheet coupled with discrete actuators makes modeling of the system very challenging. Furthermore, with typically 10 plus actuators and 10 plus sensors, the MIMO (Multi-Input Multi-Output) control problem is computationally intensive (Tyson, 1998). 


\subsection{National Ignition Facility Adaptive Optics System}

The National Ignition Facility's (NIF) adaptive optics system will be used as a representative system to help illustrate the major components utilized in this thesis, the deformable mirror, sensor and controller.

The National Ignition Facility (NIF), currently under construction by the Department of Energy at Lawrence Livermore National Laboratory, is a mega-Joule class laser and target irradiation facility for investigating the physics associated with stockpile stewardship and inertial confinement fusion (ICF). It is designed to produce 1.8 MJ of frequency-tripled radiation $(351 \mathrm{~nm})$ in 192 independently focusable beams, each with an amplifier system that has a square clear aperture of approximately $40 \mathrm{~cm} \mathrm{x} 40 \mathrm{~cm}(15.7 \mathrm{in} \mathrm{x}$ 15.7 in.). These amplifiers have aberrations that contribute to the net wavefront and affect both conversion efficiency of the frequency converters and the size of the focus spot on the target. An adaptive optics system is utilized to address static aberrations in the main beam line and precorrect dynamic aberrations induced in the main power amplifiers so that NIF can meet requirements for its conmenten efficiency and focus spot size (Winters, et al, 1998). The adaptive optic system comprises a deformable mirror, wavefront sensor, reference source and controller electronics, and one is depicted in Figure 2.1 for a single beam line (Zacharias, et al, 1998).

Next, using Figure 2.1, the path of the NIF beam is first summarized, and then the adaptive optics functions will be discussed. The NIF preamplifier $1 \omega(1.053 \mu \mathrm{m})$ beam, where $\omega$ is the frequency of the laser, enters the main laser chain near the focus of the transport spatial filter (TSF), directed away from the target. The beam exits the filter as a collimated beam that passes through the boost amplifier heading towards the laser main amplifier cavity. A Pockel cell is set to allow the beam to enter the cavity, where it makes four passes through the main amplifier before the Pockel cell is switched to allow the beam to exit. The beam then exits the cavity, passes through the boost amplifier and the TSF and 
heads towards the target chamber. The beam is frequency-converted to $3 \omega(351 \mathrm{~nm})$ at the target chamber (Zacharias, et al, 1998). The adaptive optics functions are implemented as follows. A continuous wave (cw) beam is co-aligned with the NIF beam prior to injection into the main laser. The probe beam follows the NIF beam path. A 39-actuator large aperture deformable mirror operates at the far end of the laser cavity where the beam bounces twice. At the TSF output, a tilted sampling surface reflects a small fraction of the beam towards a pick-off mirror near the TSF focus that sends the sampled beam through relays to the Output Sensor. Within the Output Sensor, a 77-lenslet Hartmann sensor measures the wavefront. The Hartmann sensor's video output is read by a frame-grabber in the wavefront control computer. The computer calculates the surface displacements to be applied to the deformable mirror to correct the wavefront aberrations in the beam.

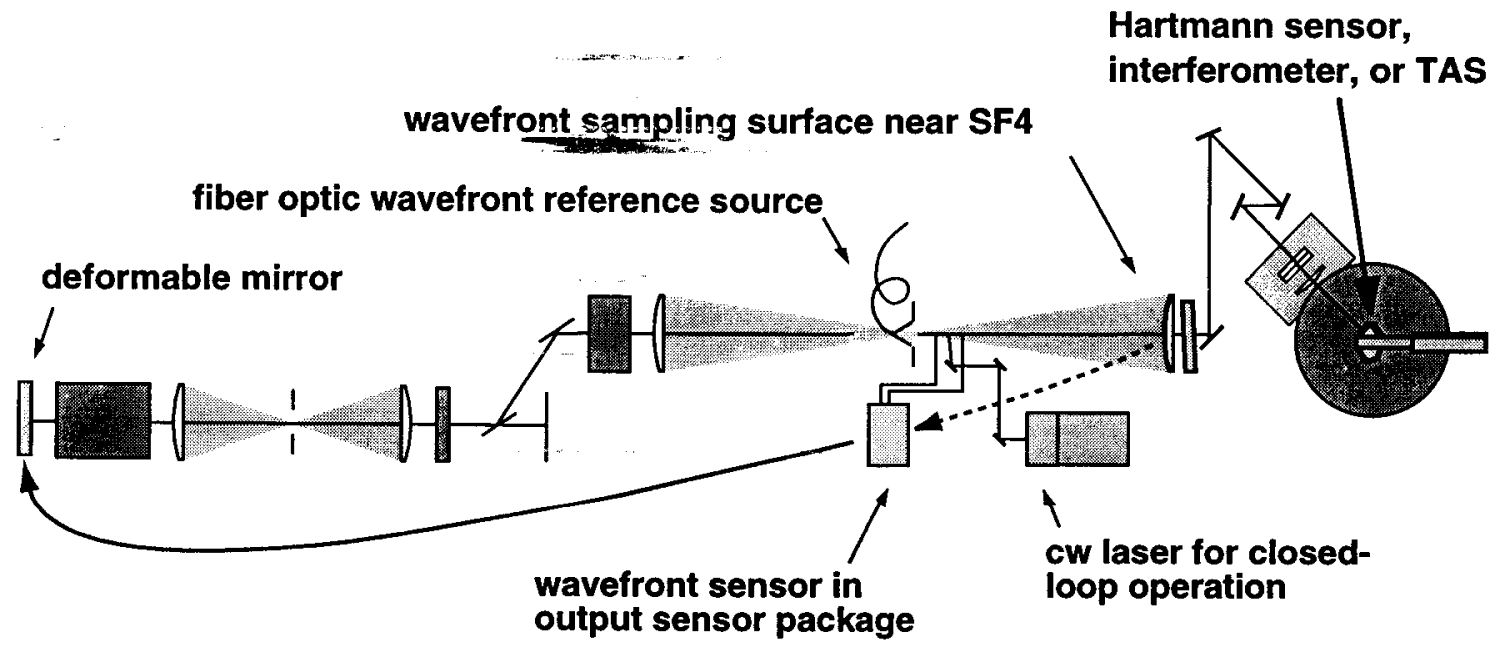

Figure 2.1 Adaptive optic components for a single chain of NIF (Zacharias, et al, 1998)

\subsection{Deformable Mirror Configurations}

The deformable mirror (DM) in general can be defined as a multiple channel continuous surface device, which utilizes discrete position actuators, discrete force 
actuators or bending moment actuators. Figure 2.2 depicts typical segmented and continuous active mirrors. Utilizing a mechanical means of deformation, these continuous surface mirrors deform to match a conjugate wavefront. The actuators can be continuous, as in a bimorph mirror, or discrete as in discrete actuator mirrors. This work will focus on the discrete actuator type deformable mirror configuration.

\section{Types of Active Mirrors}

Segmented Mirrors

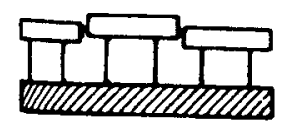

Piston only

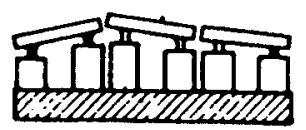

Piston plus tilt

\section{Continuous Thin-Plate Mirrors}

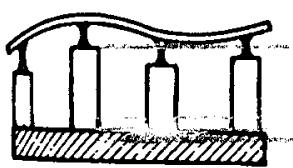

Avial actuators
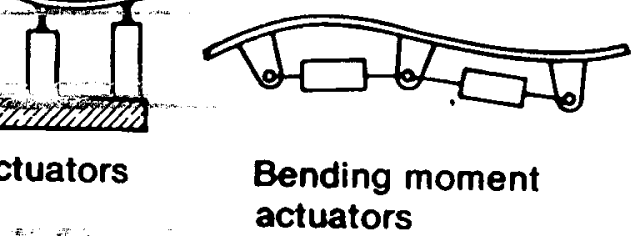

Bending moment actuators

Figure 2.2 Typical segmented and continuous active mirrors (Ealey, et al, 1991)

Discrete actuator deformable mirrors with stacked piezoelectric actuators were first developed in the late 1970's to address large stroke requirements and these are depicted in Figure 2.3. Since this early development, research has continued in many areas, e.g., actuator development to produce low voltage low hysteresis actuators, coating development to allow use in high power laser systems, and actuator spatial compactness to maximize the number of degrees of freedom per unit area for atmospheric applications (Tyson, 1998).

Actuators for these discrete actuator deformable mirrors generally fall into one of two categories; force actuators and displacement actuators. The most common material 
used for actuators are piezoelectric ceramics (Ealey, et al, 1994). These actuators have response times of 10 to 100 microseconds (Giurgiutiu, et al, 1995). For lower frequency correction, various wormgears and dc motors, hydraulic and ballscrews drives have been used to drive mirror surfaces.

The deformable mirror surface shape is defined by each of its actuators' influence function. The influence function is defined as the surface displacement when one actuator is displaced and all others are held constant. The influence function is a function of the mirrors faceplate parameters such as thickness, modulus of elasticity and Poisson's ratio.

The NIF prototype deformable mirror, shown in Figure 2.4, is based on a discrete type actuator approach. This mirror has a clear aperture of $400 \mathrm{~mm} \times 400 \mathrm{~mm}$ (15.7 in. $\mathrm{x}$ 15.7 in.), uses thirty-nine electrostrictive actuators and has a $11 \mathrm{~mm}(0.43 \mathrm{in}$.$) thick$ Zerodur faceplate (La Fiandra, et al, 1998).

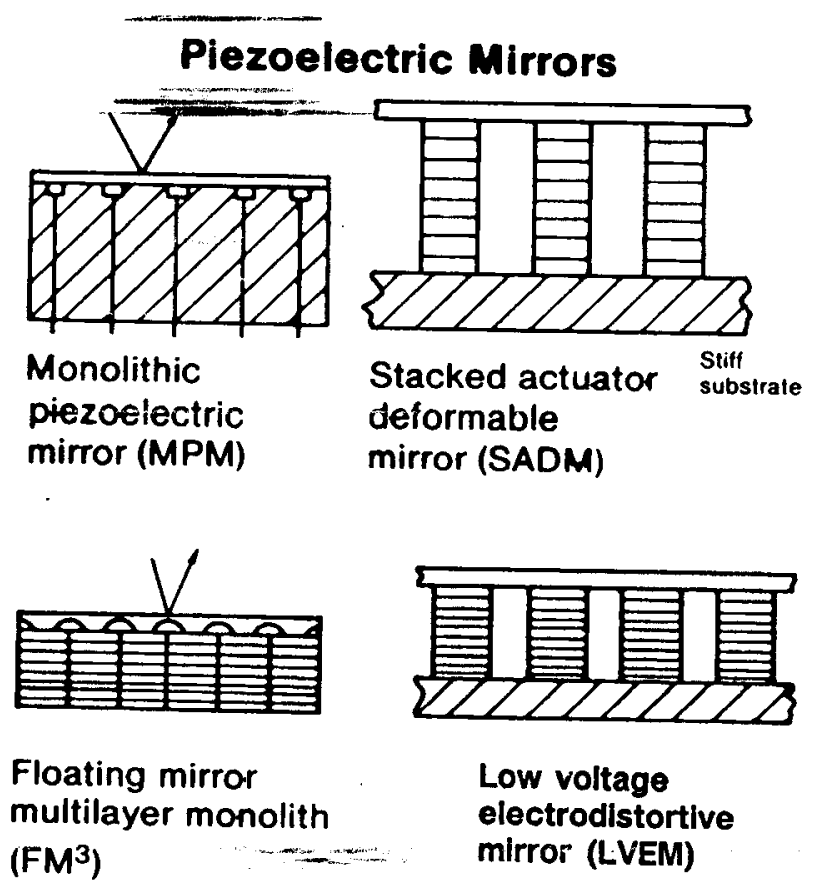

Figure 2.3 Different piezoelectric mirrors (Ealey, et al, 1991) 


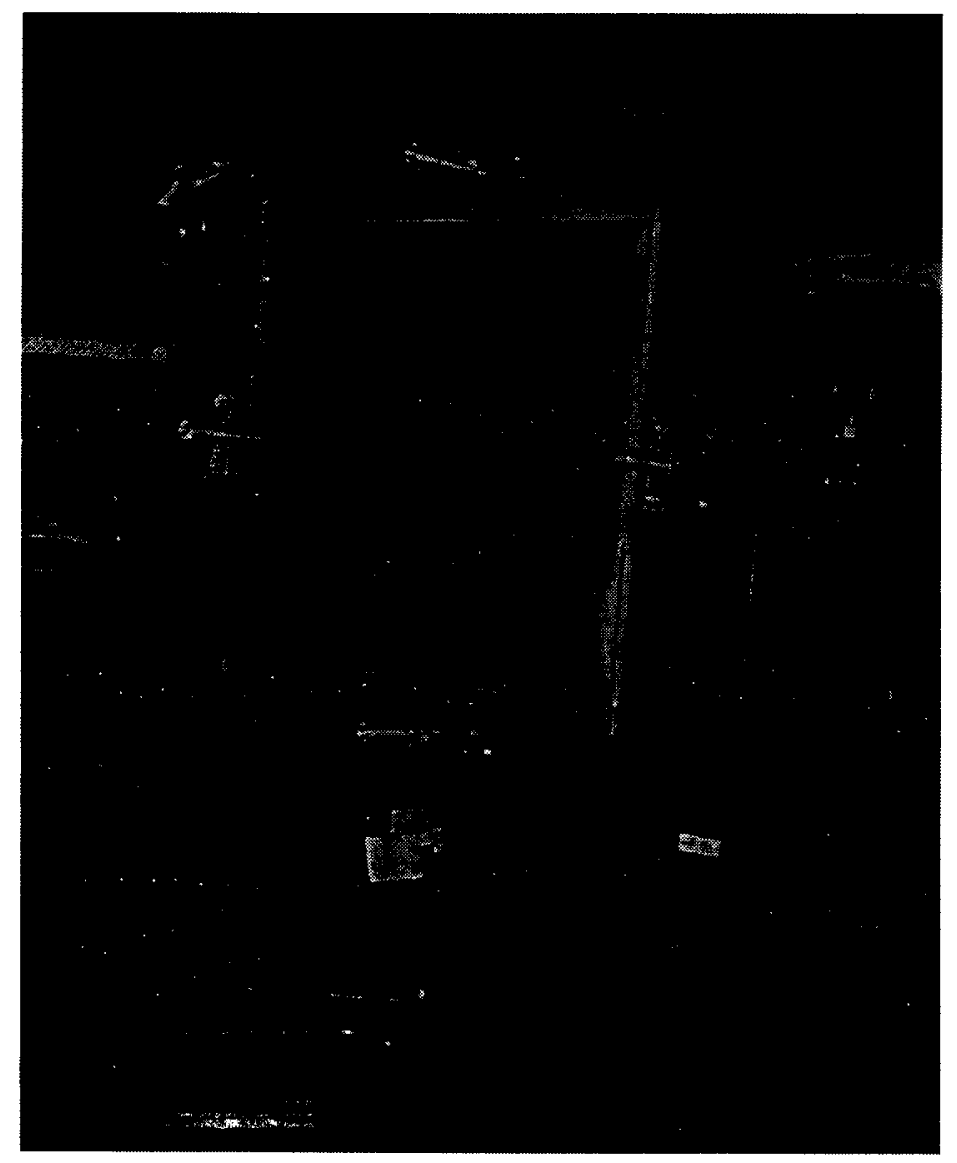

Figure 2.4 NIF prototype deformable mirror

\subsection{Sensor Configuration}

Many wavefront sensing approaches exist with sufficient spatial resolution and bandwidth to achieve closed loop operation. In general, these can be separated into a direct and indirect sensing approaches. The direct approach employs a step where there is an explicit determination of the phase, whereas in the indirect approach the phase is never calculated. The indirect approach directly translates information related to the phase into signals that are used to compensate for the wavefront. The sensors are required to sample phase at hundreds to thousands of Hertz and have sufficient spatial resolution for a given application. Additionally, the sensors are required to have very high dynamic range, allowing phase determination independent of intensity (Tyson, 1998). 
A popular direct sensing approach uses the Shack-Hartmann sensor (Tyson, 1998). This approach uses an array of lenses, where each lens acts as an aperture and produces an array of spots. With proper calibration, the position of each spot relates to the local wavefront tilt at each lens. To detect the spot, general image processing techniques are utilized. Figure 2.5 depicts the Shack-Hartmann wavefront sensing technique, showing spot motion for a diverging wavefront as compared to a plane wavefront. The NIF adaptive optics system utilizes the Shack-Hartmann sensor approach and a typical output for a flat wavefront input is shown in Figure 2.6.

\subsection{Controller Configuration}

In general, the adaptive optics controller is a multi-input multi-output configuration. The input portion of the controller utilizes information from the sensor sampling the wavefront, a processor for any control law implementation, and lastly, associated drive power amplifiers to drive the actuators.

Figure 2.7 depicts aminational block diagram of the NIF wavefront controller configuration and is described as follows. Initially, a reference source is utilized to generate a reference spot position file. Then, a probe beam is injected and the wavefront system is calibrated by an on-line procedure. Each of the 39 actuators is individually and sequentially displaced relative to the best flat condition. From this information, a gain matrix relating actuator movement to Hartmann spot movement is developed. Following calibration, the loop is closed and the Hartmann spot error is driven to zero based on the control law. Prior to a shot sequence, an additional Hartmann offset file is utilized to generate a different error vector. This additional offset file represents the uncorrected aberrations measured on the previous shot. Using these offset files, the surface shape of the deformable mirror is set to the conjugate of the expected aberrations at shot time.

The control system is designed to operate with a closed loop bandwidth of 1 Hertz. This is achieved by sampling the probe beam at 30 Hertz with the wavefront sensor. The 
sensor is output through standard RS-170 video which is read by a frame grabber. This image is read by a SPARCengine AXI computer which calculates the centroids for all 77 lenslet spots and calculates the associated error vector. This information is then sent to the controller which calculates the least squares actuator displacements (Zacharias, et al, 1998).
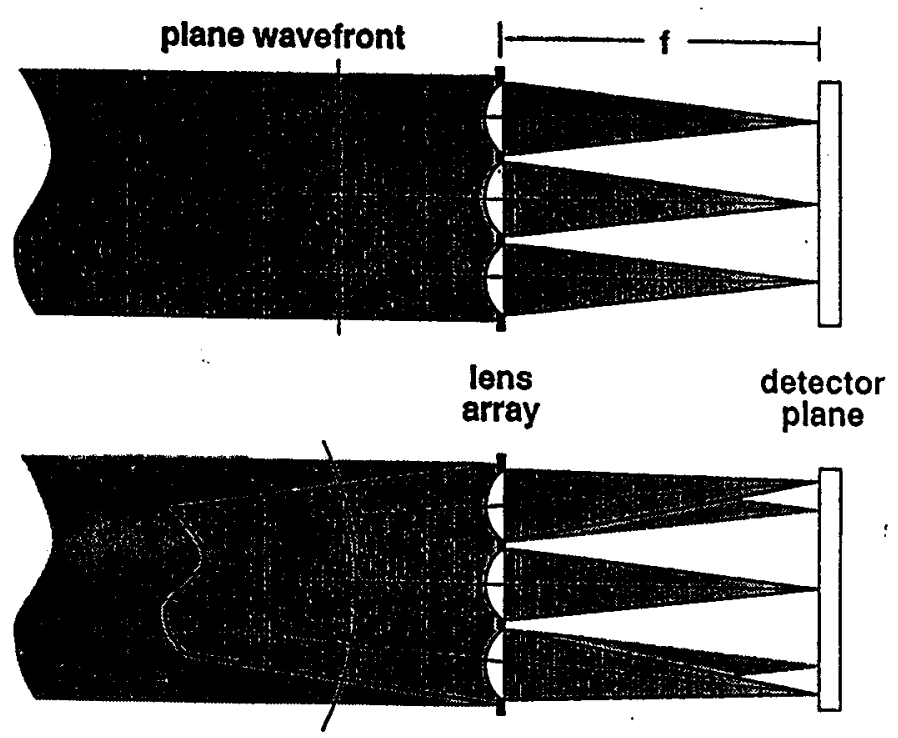

dlverging wavefront

Figure 2.5 Shack-Hartmann wavefront sensor technique 


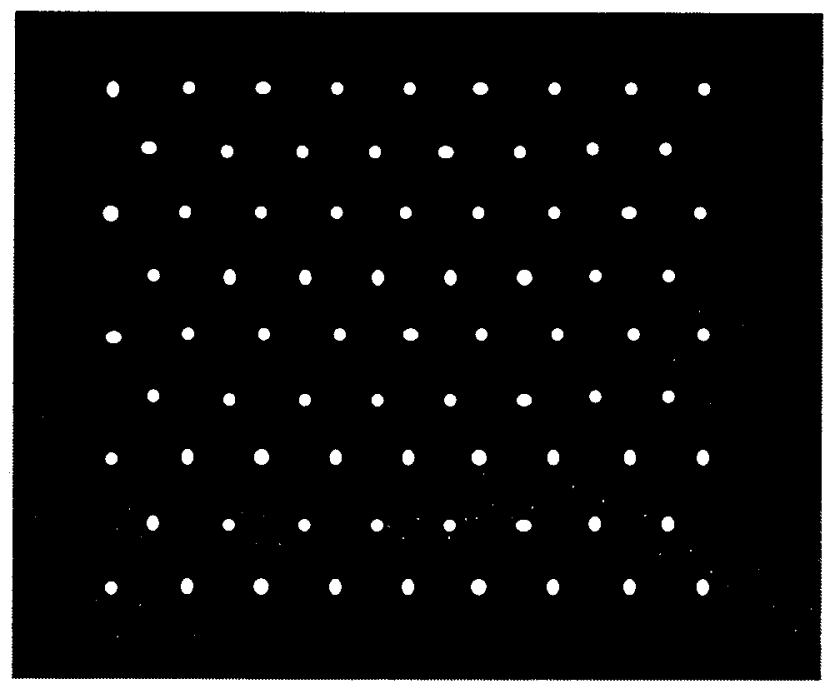

Figure 2.6 Typical spot pattern from Shack-Hartmann sensor (77 lenslets)



Figure 2.7 Controller configuration for NIF A/O system (Zacharias, et al, 1998) 


\subsection{Conclusion}

In this chapter, the field of adaptive optics was described, which included detailed descriptions of the deformable mirror, sensor and controller technology. For a specific example, the National Ignition Facility adaptive optics system components and functionality were described. Furthermore, a general overview covering adaptive optics development, development participants and focus areas was presented. The deformable mirror details focused on the continuous discrete type and sensor details focused on the Shack-Hartmann type. 


\section{CHAPTER 3 - STATIC SURFACE SHAPE MODELING}

\subsection{Introduction}

The purpose of this chapter is to develop a computer model which will facilitate the static surface shape control in the next chapter. The first part of this chapter will cover the development of a finite element model, using the commercial software package I-DEAS from Structural Dynamics Research Corporation. In the second part, experimental results will be acquired through interferometry utilizing Lawrence Livermore National Laboratory facilities. Lastly, experimental results will be compared to the finite element results and a statement of model fidelity will be established.

\subsection{Finite Element Modeling}

The purpose of this work is to develop a computer model which will represent the static surface response of a deformable mirror. An in-depth literature review covering

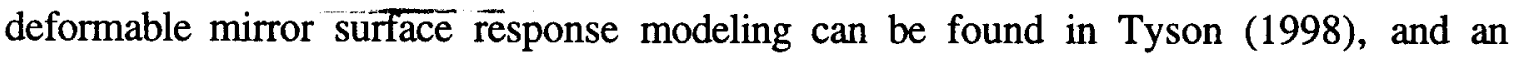
additional related Titerature review can be found in Meirovitch (1990). Authors have considered both numerical models, such as finite elements (Furber, et al, 1994a), and analytical models, such as Gaussian functions (Ealey, et al, 1991). The modeling errors associated with using most analytical functions are related to the boundary conditions along the edge and the so called pinning error effect (Hiddleston, et al, 1991). The pinning error effect occurs when all the actuators are pushed identically, and the model shows a surface that is made of bumps of equal height. This rippled surface is due to modeling errors and does not occur in the real device. Some numerical approaches, such as the finite element method, do not have these deficiencies since they maintain continuity in displacement, slope, and higher derivatives between elements. Aiter a complete evaluation of these different approaches, the finite element method was selected due to its accuracy, flexibility, and the availability of commercial software. The Structural Dynamics Research Corporation (SDRC) I-DEAS Master Series 5 (Lawry, 1997) modeling package was 
utilized to generate the finite element model of the National Ignition Facility (NIF) deformable mirror, which is shown in Figure 3.1.

A solid finite element model was developed of the NIF 39 actuator prototype deformable mirror, which included one facesheet and thirty-nine 3-DOF springs. The model was built using parabolic tetrahedron solid elements, each having 10 nodes with the overall model having 68,323 . This model is shown in Figure 3.2. To verify that the meshing errors were sufficiently small, models with mesh sizes ranging from 26,000 nodes to 350,000 nodes were developed and solved using the same boundary conditions.

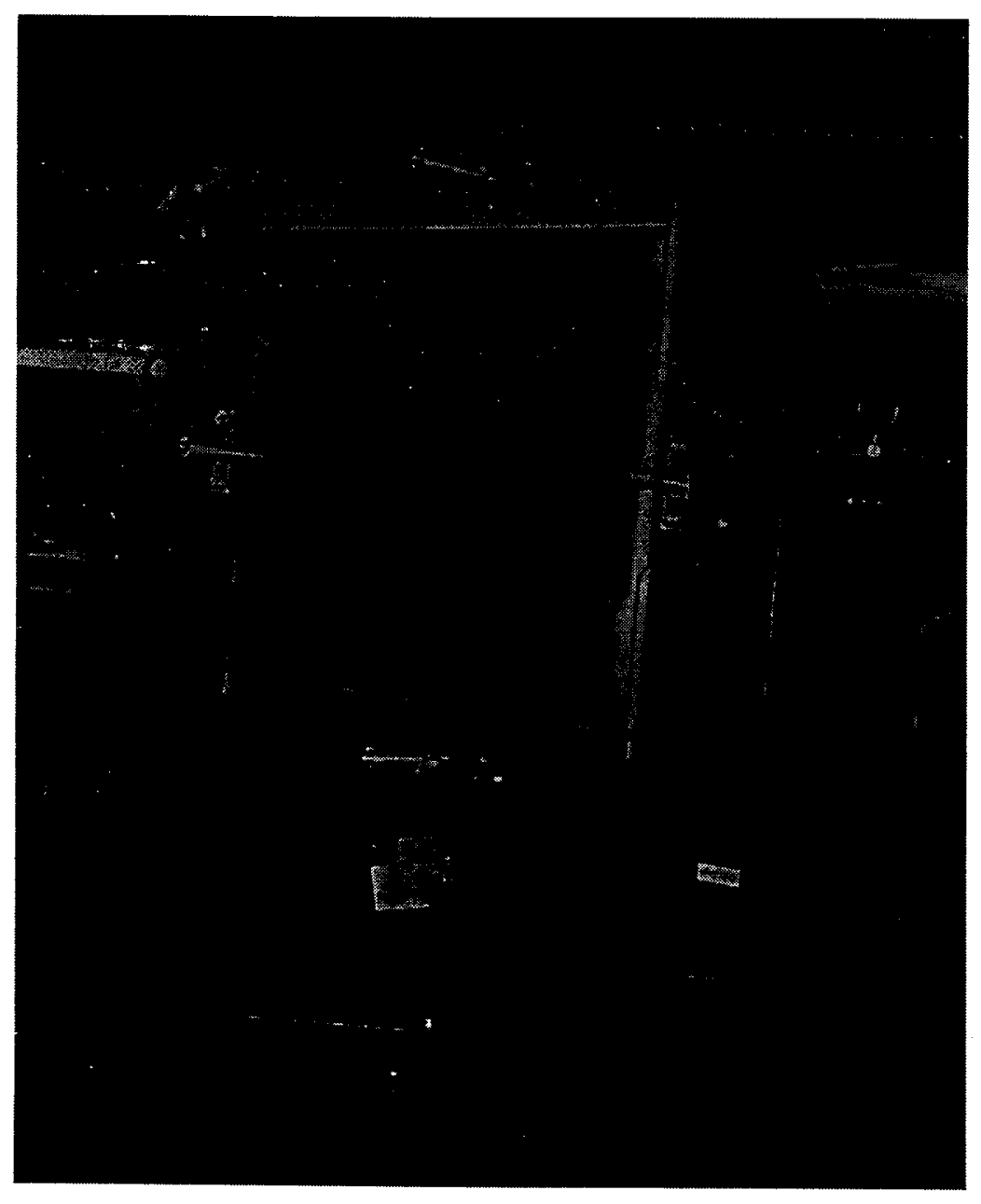

Figure 3.1 National Ignition Facility prototype deformable mirror 
This approach follows standard finite element mesh refinement techniques and will produce an assessment of the meshing error (Lawry, 1997, Russell, 1996). The boundary conditions were free edges, constrained springs, and a force applied at actuator number 20 . The selection of this boundary condition set is justified due to the symmetry in the system. Using surface displacement as the performance metric, approximately ninety-nine percent asymptotic convergence was achieved, as compared to the 350,000 node model, after reaching approximately 68,000 nodes. This convergence indicates a mesh density that is sufficient to capture the displacement spatial frequencies in the model. Therefore, it is concluded that a ninety-nine percent convergence is sufficient, based on uncertainties associated with other aspects of the modeling, e.g., material properties and boundary conditions, memory capacity limitations, and computational time considerations.

Figure 3.3 is a schematic of the mirror with the 39 actuator locations noted. With the model complete, surface shape responses (influence functions) were calculated for twelve of the thirty-nine actuators at locations $20,21,24,25,27,28,31,32,34,35,38$, 39. This data was converted to a $32 \times 32$ point grid evenly spaced over the $400 \mathrm{~mm} \times 400$ $\mathrm{mm}$ (15.75 in. $\mathrm{x} 15.75$ in.) clear aperture (i.e., laser beam footprint) for further post processing. Figure 3.3 depicts the clear aperture and Figure 3.4 depicts the $32 \times 32$ point grid projected on the mirror surface. The remaining influence functions were calculated through coordinate transformations. This is justified due to the symmetry in the system. Figure 3.5 shows an example surface response (influence function) for the center actuator, \#20.

With the model complete and the influence functions generated, the next step was to gather experimental results. These experimental results are needed to verify the accuracy of mathematical model so that it may be used for future control simulation. The magnitude of acceptable model accuracy is established by the intended use. With the ultimate goal of using this model for static surface shape control, model errors should be small compared to errors generated by the control law, the magnitude of the aberration to be corrected, etc. 
Furthermore, model errors should be comparable to uncertainties in material parameters, e.g., elastic modulus, Poisson's ratio. Lastly, once the static model is verified, small variations could be made to the model to complete sensitivity studies for design purposes.

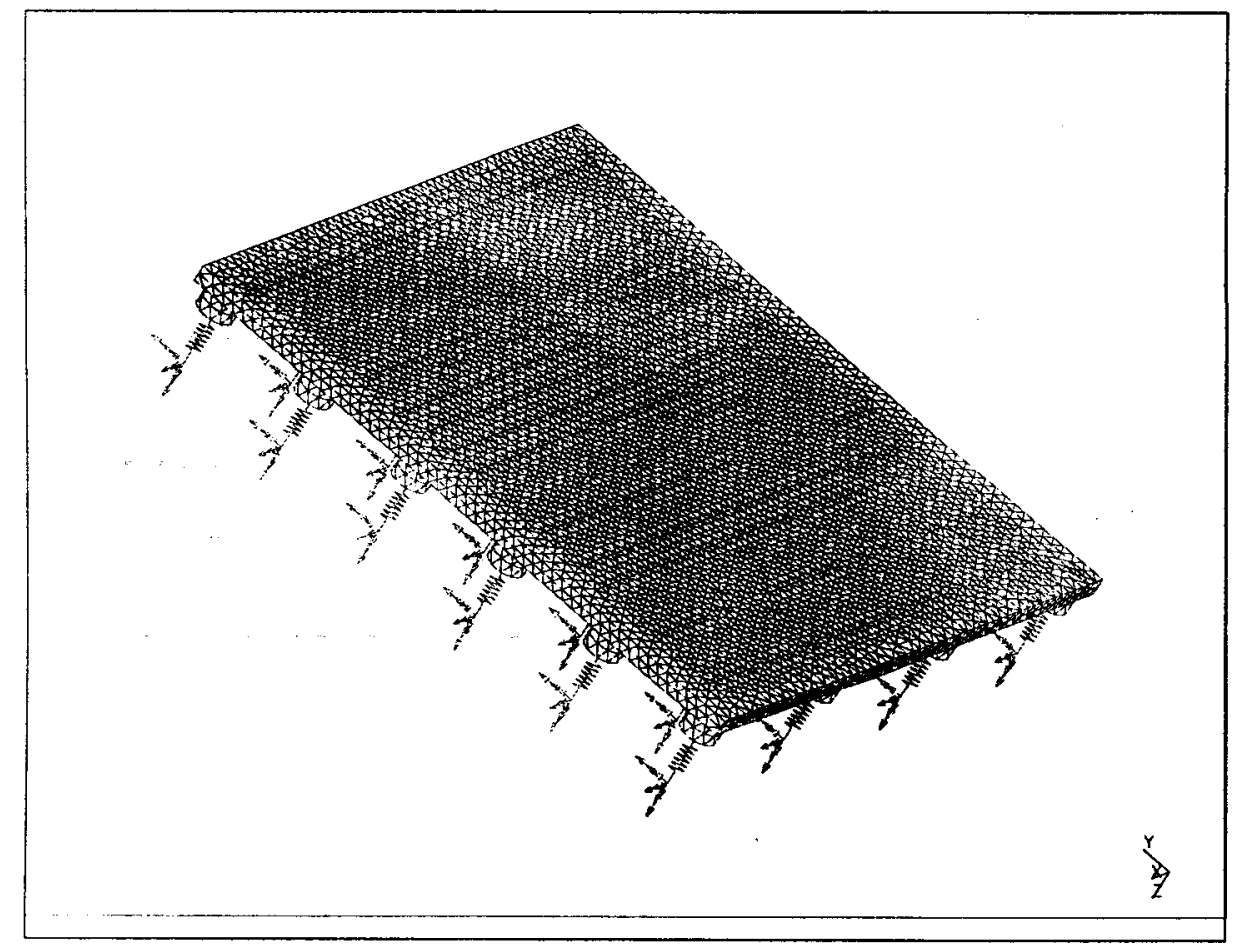

Figure 3.2 Finite element model of deformable mirror 


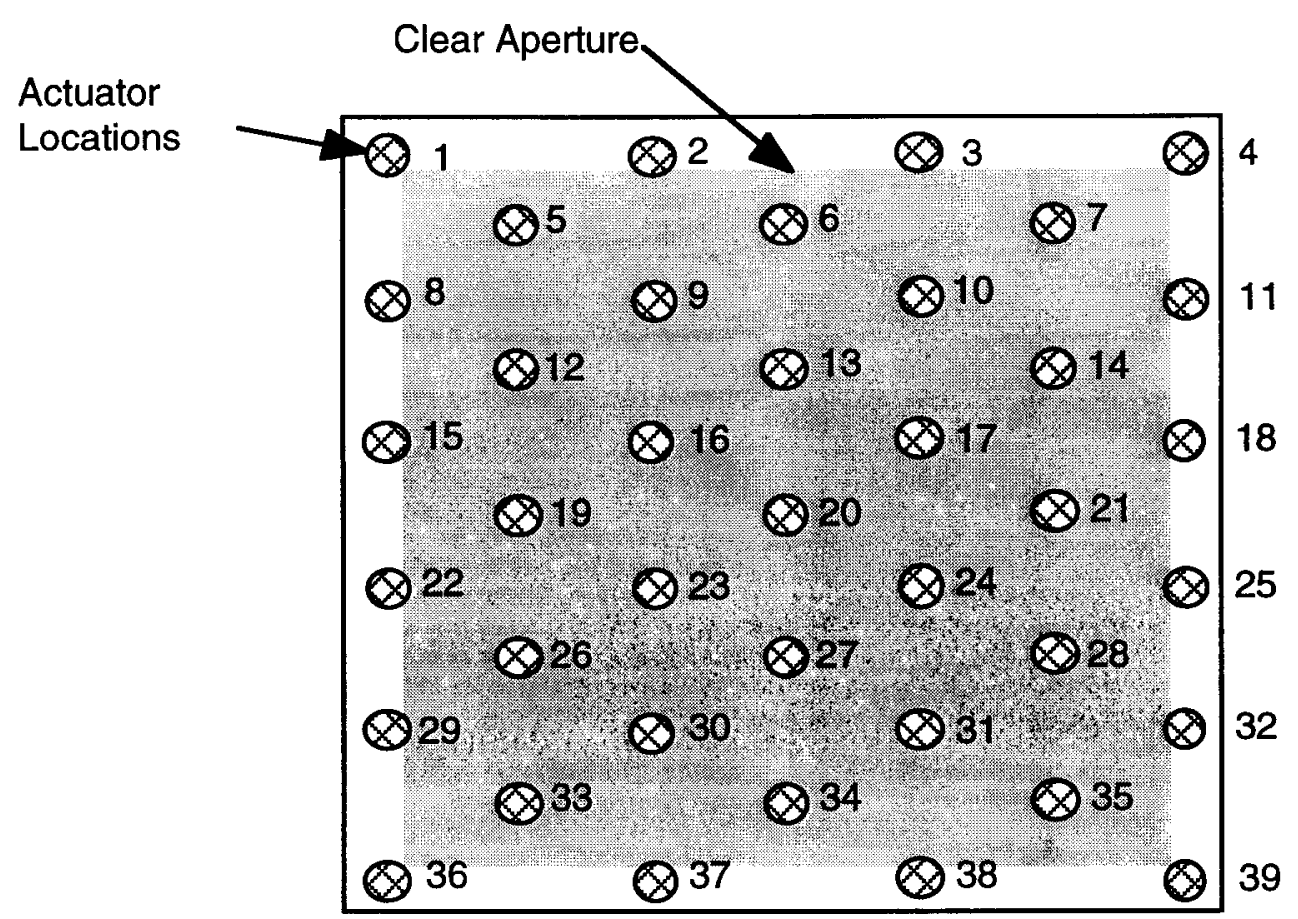

Figure 3.3 Schematic of deformable mirror including actuators and clear aperture

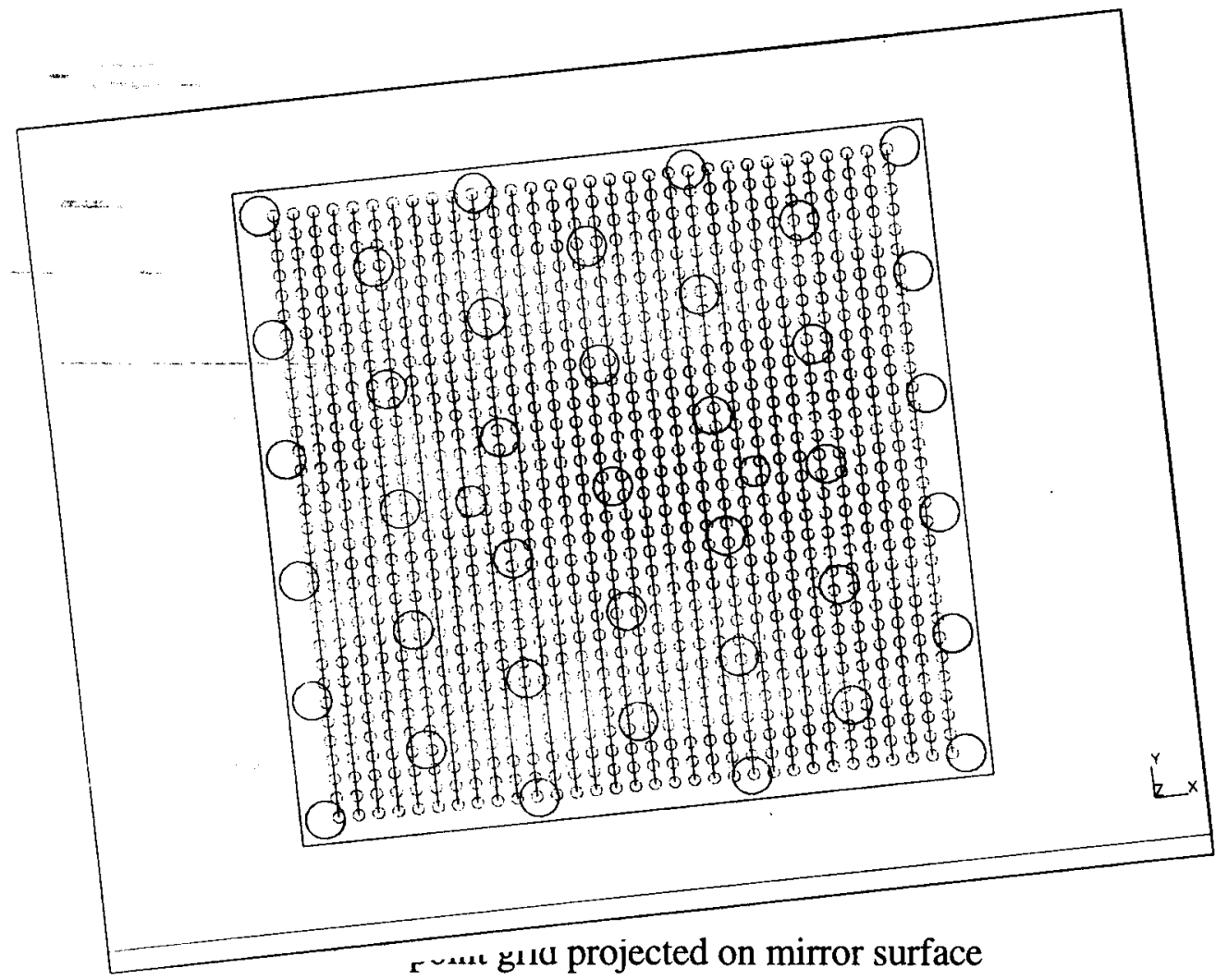




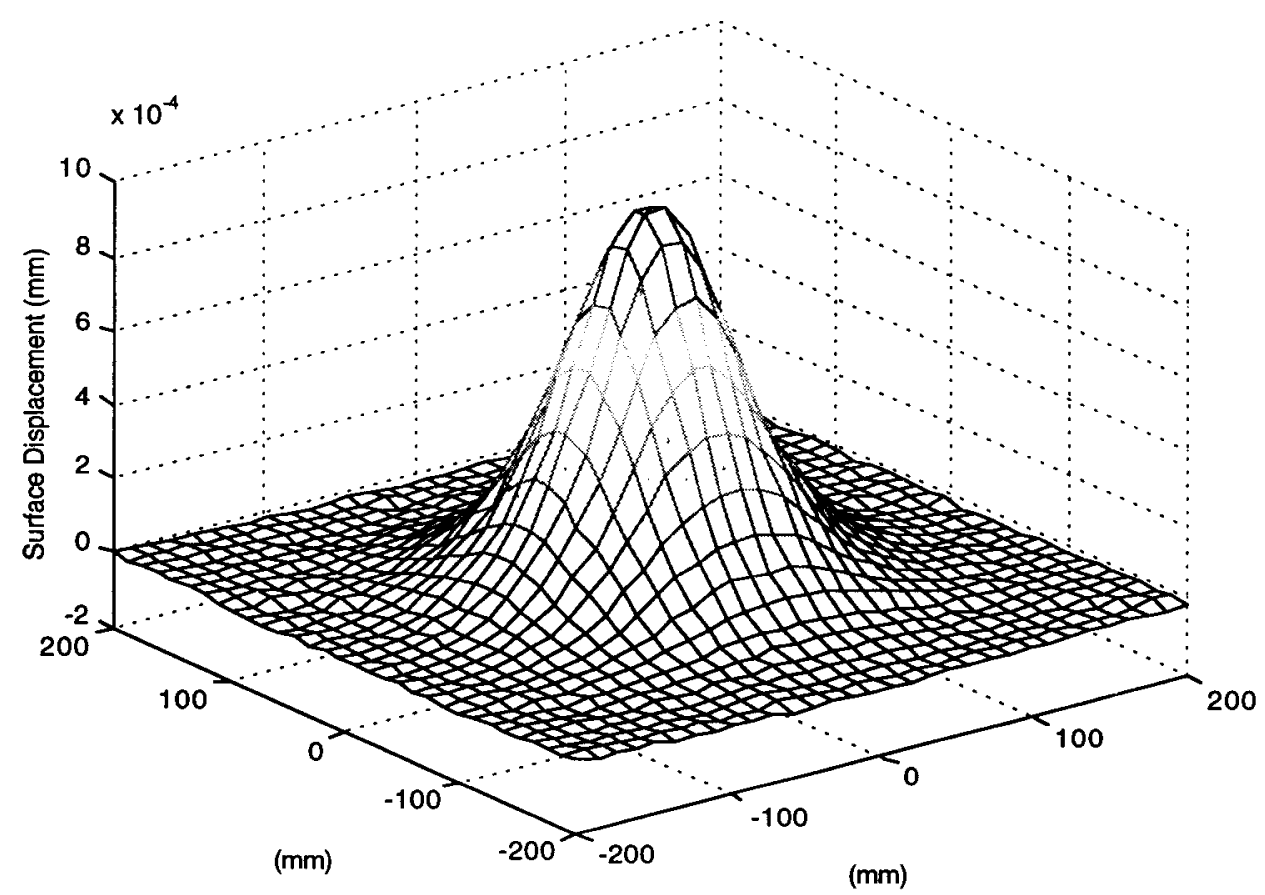

Figure 3.5 Influence function for actuator \#20

\subsection{Experimental Test}

Experimental tests were completed on the NIF 39 actuator deformable mirror in order to validate the model developed in the previous section. The tests utilized a 610 millimeter (24 inch) aperture Fizeau interferometer system at Lawrence Livermore National Laboratory (LLNL), which was specially developed to characterize the performance of the NIF adaptive optics system. The system optical components are attached to a large granite table in an environmentally controlled enclosure. The interferometer system uses a commercial 101.2 millimeter (4 inch) interferometer head from Phase Shift Technologies for both system performance measurement and to supply the probe (YAG) beam for wavefront control. The interferometer layout, as well as a photograph of the actual system, is shown in Figure 3.6. Next, using Figure 3.6, the path of the beam will be summarized. The YAG beam from the commercial interferometer head returns a portion of its beam at the 
transmission flat before the beam passes through a splitter and is expanded by a high quality Keppler telescope. The expanded beam reflects off the deformable mirror (or reference flat) and returns through the telescope. On the return path, the splitter sends a portion of the beam to the Shack-Hartmann sensor. The reference flat replaces the deformable mirror for a reference measurement used to subtract out aberrations (bias errors) in the interferometer path added by the splitter, fold mirrors, and telescope. This approach requires the reference flat surface to be extremely high quality. The wavelength of the probe beam is 1.053 micrometers and the detector is a CCD camera with a $512 \times 488$ pixel array, which results in a resolution of approximately 1.3 millimeters per pixel (Zacharias, et al, 1998).
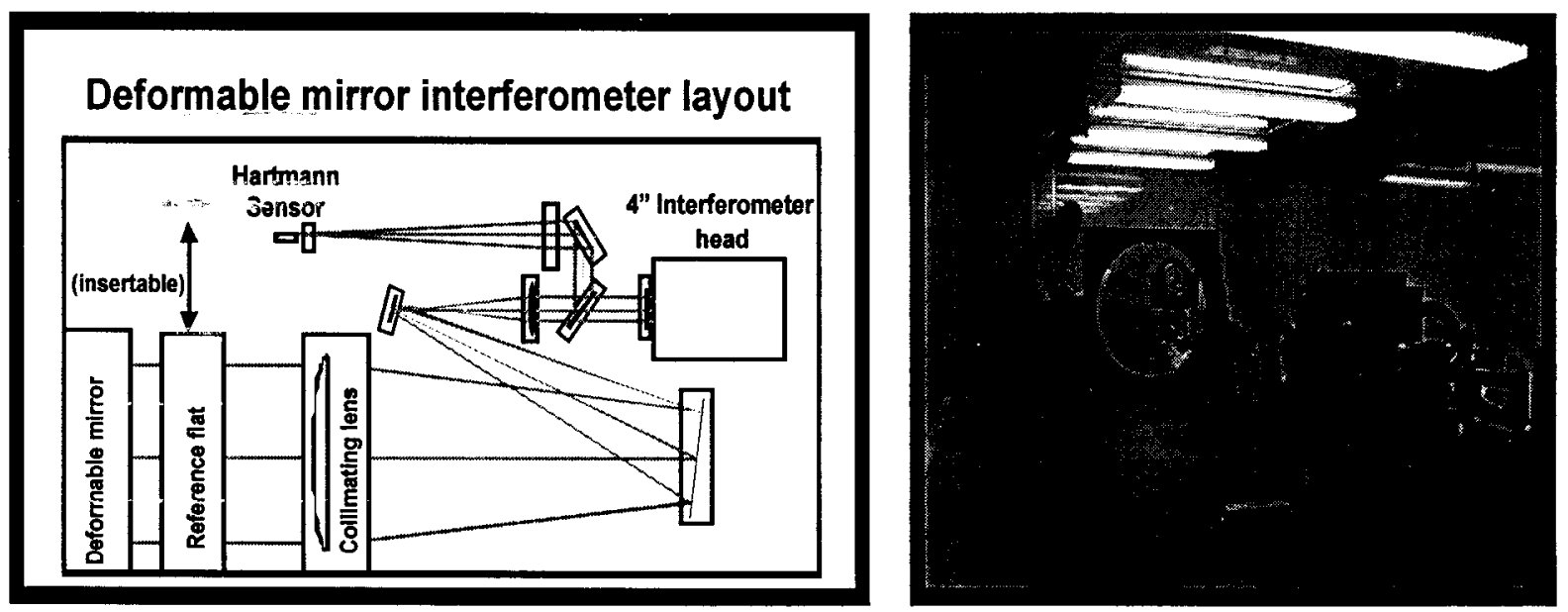

Figure 3.6 Interferometer schematic layout and photograph (Zacharias, et al, 1998)

As with any experiment, understanding the uncertainties and errors are very important. The large interferometer system was characterized through a series of tests, which included accuracy, repeatability, and reproducibility (Koch, 1998). These parameters are defined based on the International Organization for Standardization (ISO) as follows (Patterson, 1994). Accuracy is defined as the closeness of the agreement between the result of a measurement and a true value of the measurand. Repeatability is defined as 
the closeness of the agreement between the results of successive measurements of the same measurand carried out under the same conditions of measurement. Reproducibility is defined as the closeness of the agreement between the results of measurements of the same measurand carried out under changed conditions of measurement. For these specific tests, repeatability measurements were taken over a few minutes and reproducibility measurements were taken over approximately one hour. The most important characterization for verifying the mathematical model is repeatability, since two measurements are taken and then subtracted to get the experimental influence function. This is accomplished by taking a measurement of the deformable mirror at a nominal voltage condition and then repeating the measurement after increasing the voltage on a single actuator. The difference between these two measurements gives the actuator influence function. Table 3.1 summarizes the overall characterization test results, which shows a peak-to-valley accuracy of 0.2 micrometers ( 7.9 microinches), repeatability of 0.028 micrometers (1.1 microinches), and reproducibility of 0.057 micrometers (2.2 microinches). The results in Table 3.1 were generated using Phase Shifts' root-meansquare (RMS) and peak-to-valley (P-V) calculations that utilized a 512 x $488 \mathrm{CCD}$ camera pixel array. On the basis of these test results, it is apparent that a static bias error exists. As discussed earlier, this is due to the fabrication and mounting errors associated with the optical components. Although the bias error is relatively large, since it is static it can be subtracted from the measurement results. Therefore, as stated earlier, the repeatability error is the most important characterization for verifying the mathematical model.

Utilizing the large interferometer system, the NIF 39 actuator deformable mirror was tested by displacing each actuator sequentially, and recording the interferometric data using the Phase Shift interferometer. This data was then converted to displacement data using Phase Shifts' post processing software. An example of this data, after being interpolated to a $32 \times 32$ point grid from Phase Shifts' CCD grid of points, is shown in Figure 3.7 (Sacks, 1998). 


\begin{tabular}{|c|c|c|}
\hline Test & RMS Result & Peak-to-Valley Result \\
\hline Accuracy & $0.034 \mu \mathrm{m}(1.3 \mu \mathrm{in})$ & $0.2 \mu \mathrm{m}(7.9 \mu \mathrm{in})$ \\
\hline Repeatability & $0.005 \mu \mathrm{m}(0.20 \mu \mathrm{in})$ & $0.028 \mu \mathrm{m}(1.1 \mu \mathrm{in})$ \\
\hline Reproducibility & $0.008 \mu \mathrm{m}(0.32 \mu \mathrm{in})$ & $0.057 \mu \mathrm{m}(2.2 \mu \mathrm{in})$ \\
\hline
\end{tabular}

Table 3.1 Summary of interferometer characterization

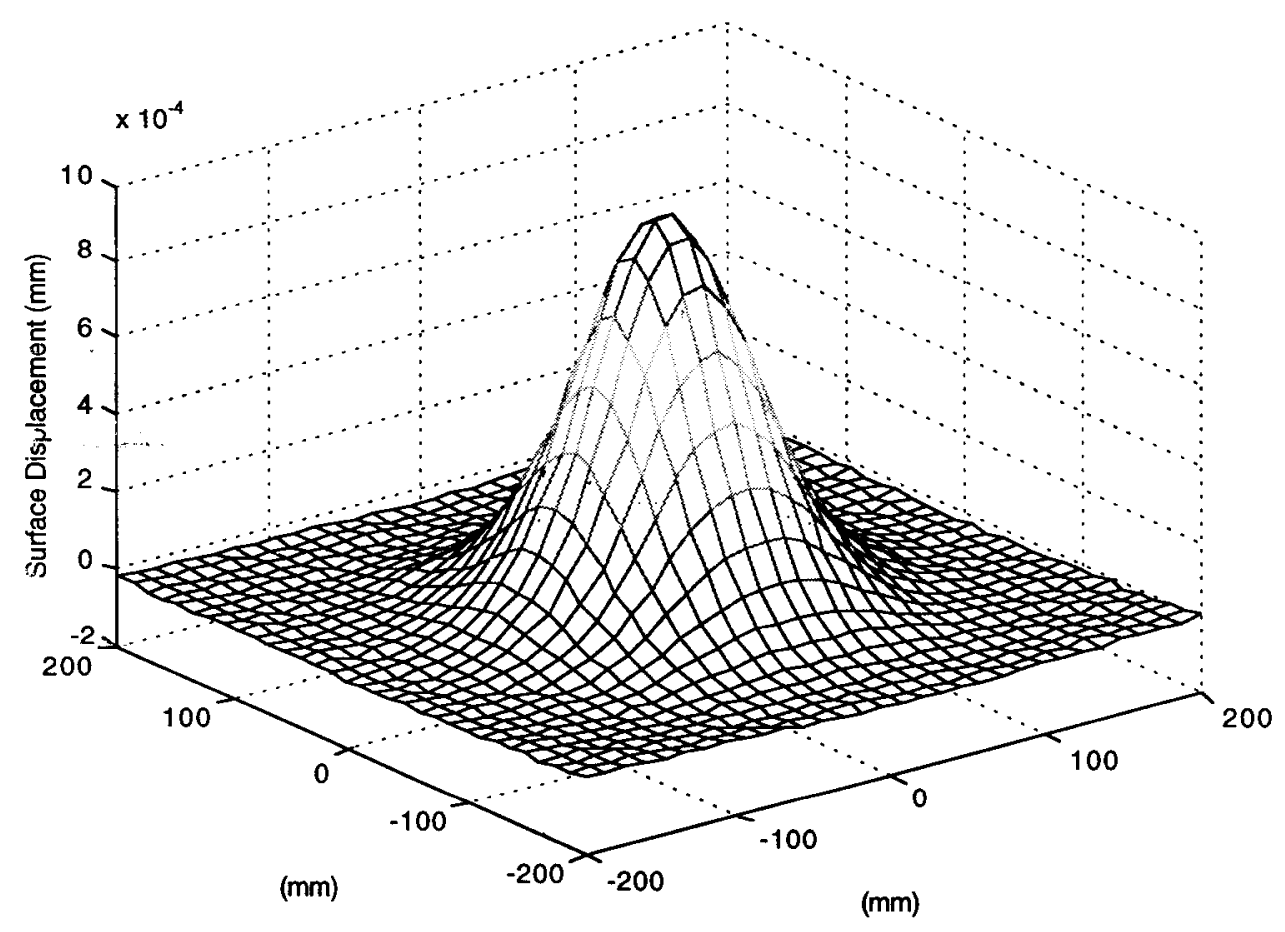

Figure 3.7 Experimental data for actuator \#20

\subsection{Model Verification}

In this section, a comparison between results generated from the finite element model and experiment will be made. This comparison will allow a statement of model 
fidelity to be made and also gives confidence in using the model for future control implementation.

Two approaches in making the comparison between the model and experimental data were made, one using a linear least squares fit (Kreyszig, 1988) approach between the model and the experimental data sets and the second is based on a normalization approach (Sacks, 1998a). Comparisons were made between influence functions 3, 4 and 20 since these give representative locations over the surface. Figure 3.3 shows the actuator numbering scheme.

The linear least squares equation can be formulated as

$$
f=\sum_{j=1}^{m} \sum_{i=1}^{n}\left(C_{j} z_{i}^{j}-q_{i}\right)^{2}
$$

where,

$m=$ \# of actuators (equal to one for this application),

$n=\quad \#$ of grid points,

$C_{j}=$ coefficient for the jth influence function,

$z_{i}^{j}=$ model surface displacement for the ith grid point and jth actuator, and

$q_{i}=$ experimental surface displacement for the ith grid point.

To find the minimum of the above equation, the partial derivative is taken with respect to the coefficient $C_{j}$ and then set to zero, which yields the following equation

$$
0=2 \sum_{i=1}^{n} z_{i}^{j}\left(C_{j} z_{i}^{j}-q_{i}\right)
$$

which can be re-written and solved for the coefficient as 


$$
C_{j}=\frac{\sum_{i=1}^{n} q_{i}}{\sum_{i=1}^{n} z_{i}^{j}}
$$

Using Equation (3.3), the fitting coefficient is calculated and then applied to the model data. Then, the difference between the experimental and model results is calculated.

The normalization method can be formulated by normalizing both the model and experimental data by each of its maximum values, respectively. These equations are formulated as

$$
\begin{aligned}
& Q_{i}=\frac{q_{i}}{q_{\max }} \text { and } \\
& Z_{i}=\frac{z_{i}}{Z_{\max }}
\end{aligned}
$$

where,

$i=\quad 1 \ldots \ldots . n$

$n=\quad$ \# of grid points,

$Q_{i}=$ normalized experimental surface displacement for the ith grid point,

$q_{i}=$ experimental surface displacement for the ith grid point,

$q_{\max }=$ maximum experimental surface displacement,

$Z_{i}=$ normalized model surface displacement for the ith grid point,

$z_{i}=$ model surface displacement for the ith grid point, and

$z_{\max }=$ maximum model surface displacement. 
Using Equations (3.4) and (3.5), the normalized model and experimental surface displacement data are calculated and then subtracted.

The least squares method was compared to the normalization method results and it was decided to use the normalization method for all comparisons due to the difficulty in aligning the experimental data. Results for selected actuators can be seen in Table 3.2, which shows actuator number and percent difference between model and experiment relative to the maximum surface displacement. Figures 3.8, 3.9 and 3.10 depict differences between model and experimental results for actuator \#20. It should be noted that better translation alignment between experimental and model data would most likely reduce the percent difference. This translation alignment error is evident in Figure 3.8.

\begin{tabular}{|c|c|c|c|}
\hline $\begin{array}{c}\text { Actuator } \\
\text { Number }\end{array}$ & $\begin{array}{c}\text { Maximum } \\
\text { Displacement }(\mu \mathrm{m})\end{array}$ & $\begin{array}{c}\text { Max. Difference } \\
\text { between Experiment } \\
\text { and Model Data }(\mu \mathrm{m})\end{array}$ & $\begin{array}{c}\text { Percent } \\
\text { Difference } \\
(\%)\end{array}$ \\
\hline 3 & 1.0 & 0.06 & $+/-6$ \\
\hline 4 & 1.0 & 0.03 & $+/-3$ \\
\hline 20 & 1.0 & 0.05 & $+/-5$ \\
\hline
\end{tabular}

Table 3.2 Percent difference between model and experimental data

On the basis of the results listed in Table 3.2, a statement of model accuracy or fidelity can be established. The peak-to-valley repeatability errors associated with the experiment, listed in Table 3.1, represent approximately 20 percent to 50 percent of the difference between the model and experimental data listed in Table 3.2. Additionally, the root-mean-square (RMS) repeatability errors, listed in Table 3.1, represent approximately 4 percent to 8 percent of the difference. However, since the model and experimental difference errors appear to be systematic and spatially slowly varying, as seen in Figures 
3.9 and 3.10, reasonable and accurate conclusions can be made. Furthermore, as discussed earlier, material property uncertainties can be on the order of 5 to 10 percent. Other authors (Furber, et al, 1994b) have reported difference errors for similar models on the order of 8 percent of maximum surface displacement using finite element models. Therefore, the above data strongly supports the verification of the static model.

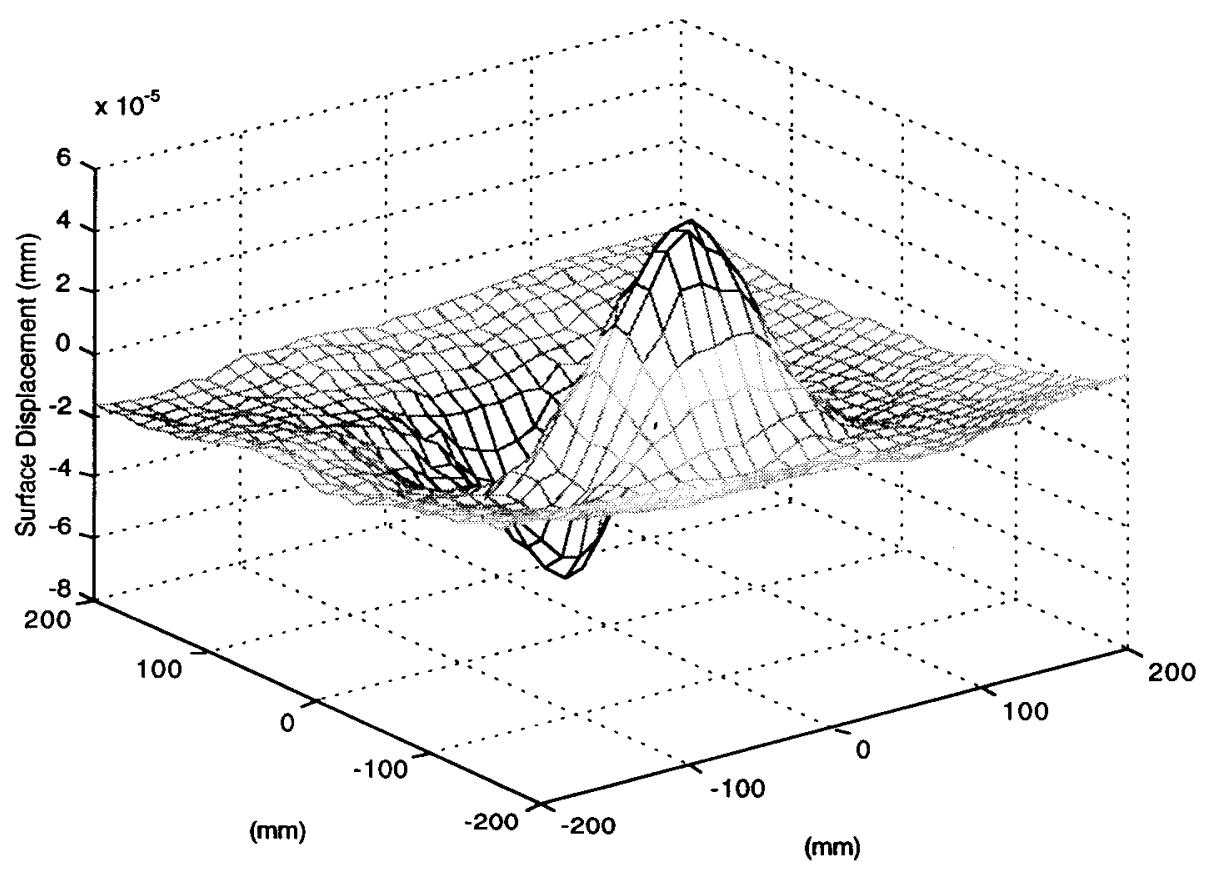

Figure 3.8 Difference between model and experimental data for actuator \#20 


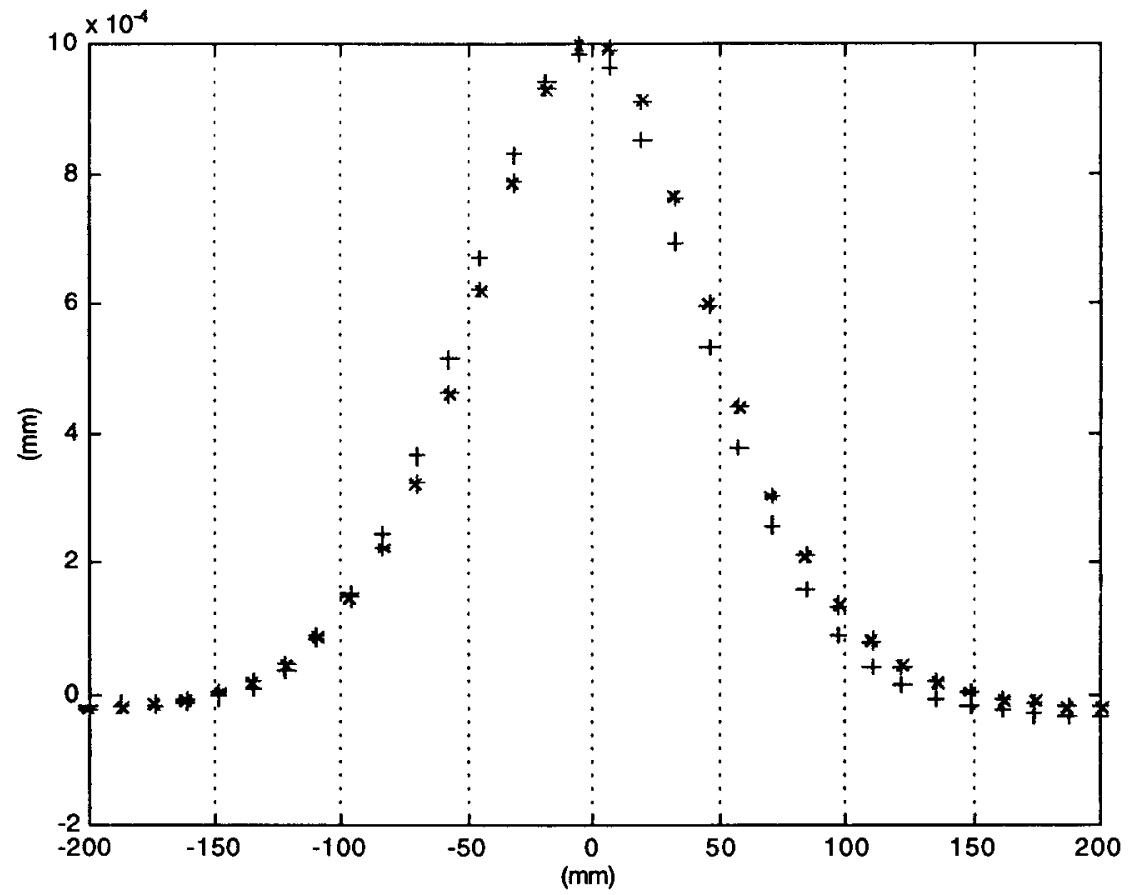

Figure 3.9 Line plot of model $(*)$ and experiment $(+)$ for Actuator \#20 (y-direction)

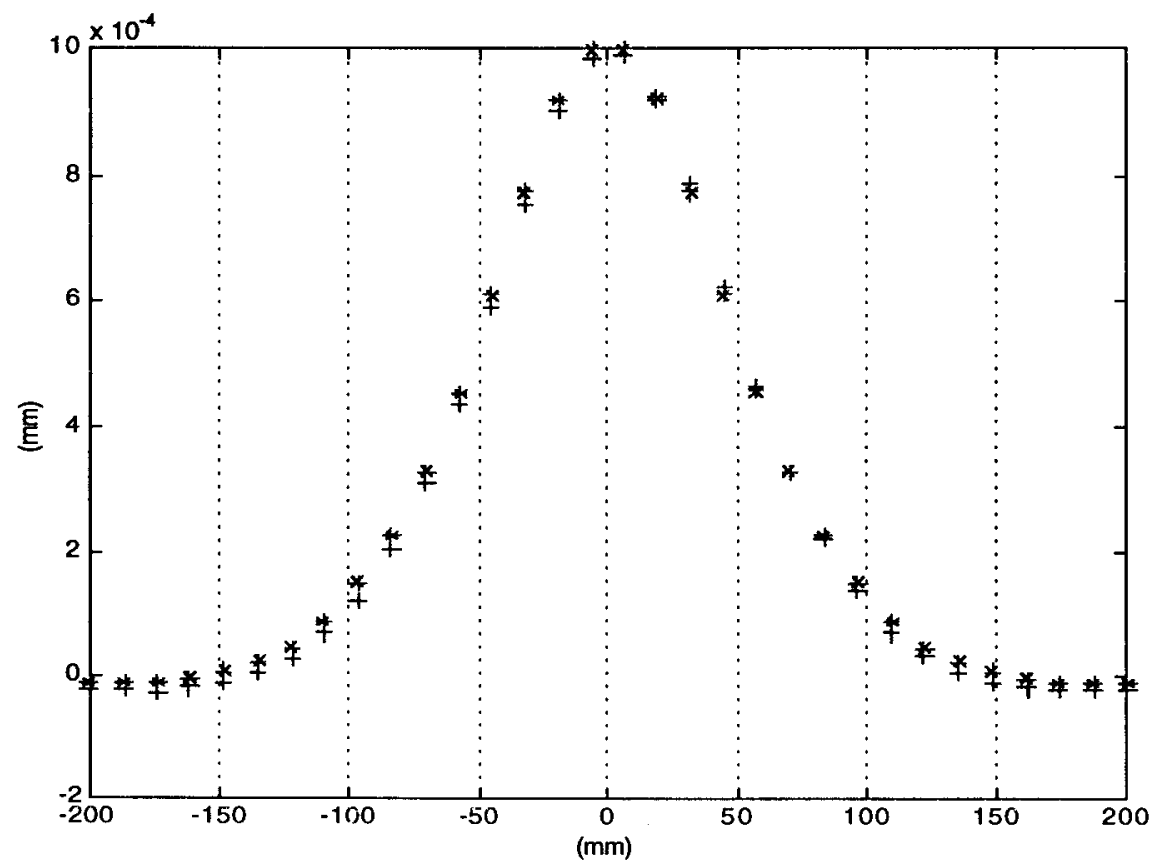

Figure 3.10 Line plot of model $(*)$ and experiment $(+)$ for Actuator \#20 (x-direction) 


\subsection{Conclusion}

In this chapter, a validated computer model was developed which will be utilized for static surface shape control in the next chapter. The first part of this chapter covered the development of a finite element model, using the commercial software package I-DEAS from Structural Dynamics Research Corporation. The finite element method was selected over other approaches, such as analytical methods, due to its accuracy, flexibility and the availability of commercial software. In the second part, experimental results were acquired through interferometry which described the surface deformation of the NIF deformable mirror. The experimental setup and errors associated with the experimental results were discussed in detail. Lastly, experimental results were compared to the finite element results utilizing a normalization approach and a statement of model fidelity was established. Model and experimental peak-to-valley result differences were a maximum of $+/-6$ percent, which is comparable to material property uncertainties. 


\section{CHAPTER 4 - STATIC SURFACE SHAPE CONTROL}

\section{1 - Introduction}

The purpose of this chapter is to investigate geometrical or static surface shape control utilizing the finite element model developed and validated in Chapter 3. In addition to developing a static control model, this chapter will demonstrate the value of using a validated plant model versus using less accurate models. The National Ignition Facility (NIF) adaptive optics configuration will be utilized for a specific example. The first section will cover formulation of the plant model, which utilizes the output from the finite element model. The second section covers development of a sensor model, which is a finite difference matrix representation of a Shack-Hartmann sensor. The Shack-Hartmann sensor, as described in Chapter 2, measures wavefront aberrations which are directly proportional to the average tilt in the wavefront across the lenslet. The third section covers development of the controller model, which utilizes a typical least squares control algorithm. With the control model developed, simulations are completed to show the general functionality of the control to correct for typical NIF wavefront aberrations. Lastly, performance comparisons are made between the finite element static plant model and a Gaussian plant model.

\subsection{Control System Description}

The general block diagram describing the geometrical control approach is shown in Figure 4.1. The remaining portion of this chapter will develop and discuss the controller matrix, plant matrix and sensor matrix represented by the notation in Figure 4.1, where,

$\tilde{r}=$ reference input vector $(\mathrm{n})$,

$\tilde{e}=$ tracking error vector $(\mathrm{n})$,

$\tilde{G}_{c}=$ controller matrix $(\mathrm{mxn})$, 
$\tilde{u}=$ input vector $(\mathrm{m})$,

$d \tilde{u}=$ input disturbance vector $(\mathrm{m})$,

$\tilde{G}_{p}=$ plant matrix $(\mathrm{rxm})$,

$d \tilde{y}=$ output disturbance $(\mathrm{r})$,

$\tilde{y}=$ plant output vector $(r)$,

$\tilde{n}=$ sensor noise vector $(r)$, and

$\tilde{G}_{S}=$ sensor matrix (nxr).

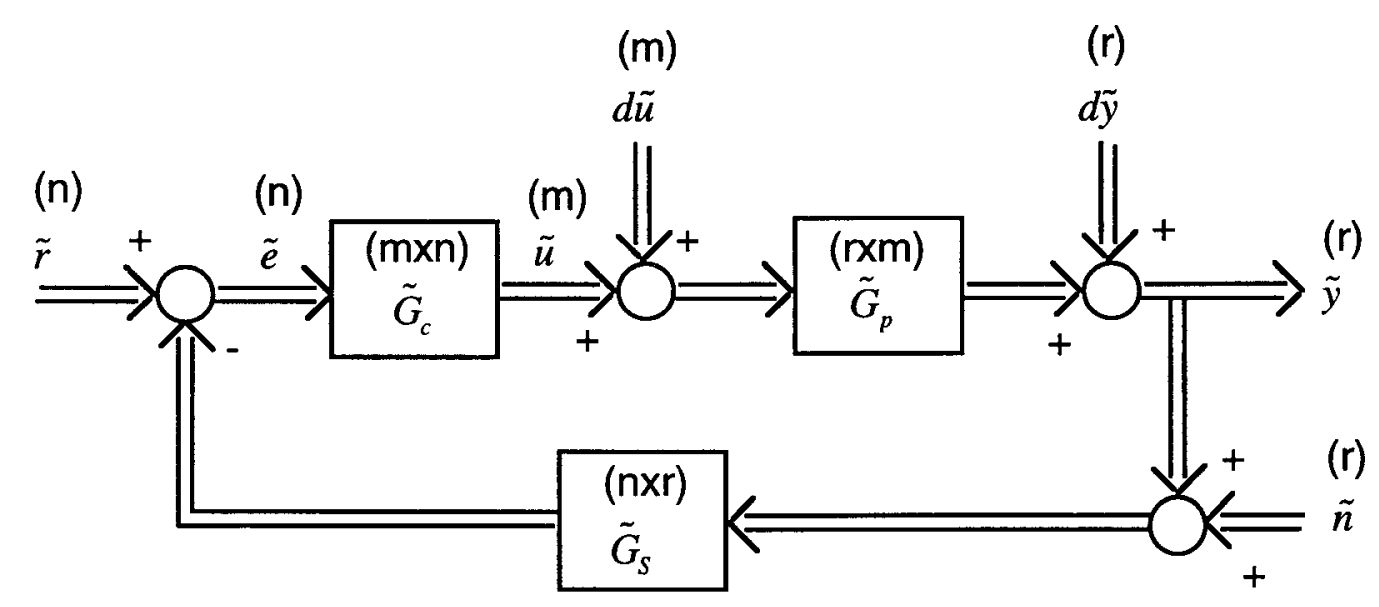

Figure 4.1 General MIMO block diagram

\section{3 - Plant Model}

The plant matrix, $\tilde{G}_{p}$, is modeled as a static or geometric system and is represented as a non-square matrix which maps actuator input space to surface displacement output space. This matrix is also referred to as the full influence function matrix (Furber, et al, 1994a). The finite element model output, developed and validated in Chapter 3, is utilized as the plant model. Figure 4.2 shows a schematic of the deformable mirror surface with actuator positions and projected lenslets of the Shack-Hartmann sensor. In general, the 
plant model can be generated numerically, such as with the finite element method, analytically, such as a Gaussian function, and/or experimentally using an interferometer or other surface displacement measurement device. The input-output relationship for the plant is defined as

$$
\tilde{y}=\tilde{G}_{p} \tilde{u} .
$$

The plant matrix, $\tilde{G}_{p}$, can be decomposed into a product of two matrices (Furber, et al, 1994a), $\tilde{J}$, the plant compliance matrix and, $\tilde{K}$, the plant stiffness matrix, where,

$$
\tilde{G}_{p}=\tilde{J} \tilde{K}
$$

The stiffness matrix, $\tilde{K}$, is defined as the mapping from actuator displacement to actuator force and the compliance matrix, $\tilde{J}$, is defined as a mapping from actuator forces to surface displacement. This is an important relationship, showing that for a given actuator spacing and configuration (i.e., the $\tilde{J}$ matrix), different stiffness matrices, $\tilde{K}$, can be formulated. Therefore, two systems with the same $\tilde{J}$ matrix are equivalent with a simple transformation of the stiffness matrix (i.e., different input vector). The next section will cover the development of the sensor model.

\section{4 - Sensor Model}

The sensor matrix, $\tilde{G}_{s}$, is modeled as a static system and is represented as a nonsquare matrix which maps displacements to average slopes. As discussed in Chapter 2, there are many different types of wavefront sensors utilized for adaptive optic control. For this analysis, the Shack-Hartmann design will be implemented, and the sensor matrix 
developed by Salmon, et al (1993) will be utilized. As described in Chapter 2, the ShackHartmann sensor uses an array of lenses (lenslet array), where each lens locally samples the incident beam and acts as an aperture and produces an array of spots. These spots are directly proportional to the intensity-weighted average tilt in the wavefront across the lenslet. To detect and track the spots, general image processing techniques are utilized. Figure 4.2 depicts the mapping of the lenslet array to the actuator positions. Next, the Shack-Hartmann sensor matrix will be developed.

There are two distinct types of lenslets, type I and type II. A type I lenslet is one that is centered on each subaperture of the deformable mirror, midway between three actuators. A type II configuration covers the lenslets that are covered over each actuator. These configurations are shown in Figure 4.3. Using Figure 4.3a, the finite difference equations for a type I are defined as

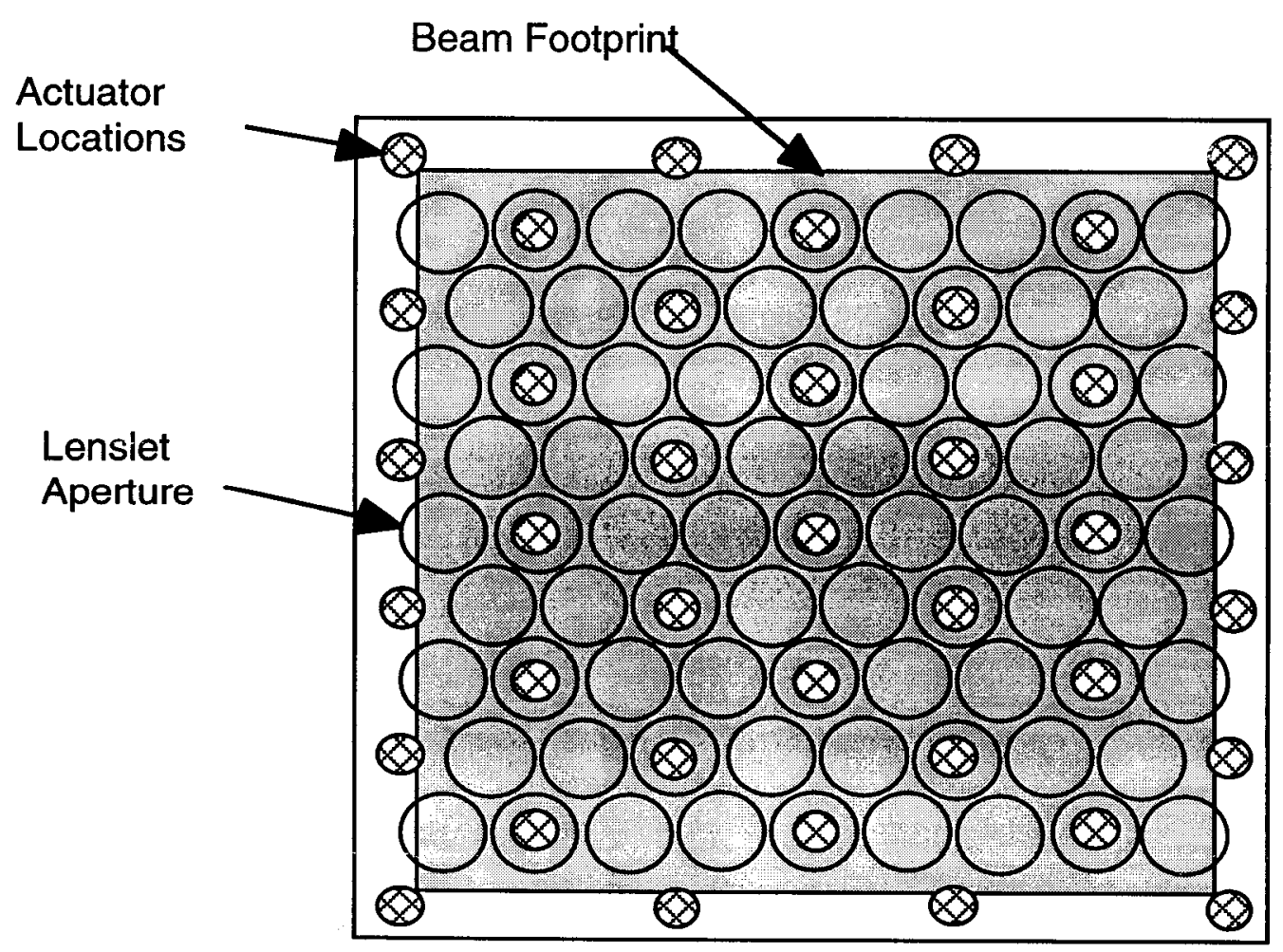

Figure 4.2 Schematic of NIF deformable mirror with actuators and projected lenslets 


$$
\begin{gathered}
r_{x}=\frac{2 z_{2}-z_{1}-z_{3}}{h \sqrt{3}} \text { and } \\
r_{y}=\frac{z_{1}-z_{3}}{h}
\end{gathered}
$$

where $h$ is the distance between actuators, $r_{x}$ and $r_{y}$ are the $\mathrm{x}$ and $\mathrm{y}$-direction slopes, respectively, and the $z_{i}$ 's are displacements at corresponding actuator locations. Using Figure $4.3 \mathrm{~b}$, the type II finite difference equations are defined as

$$
\begin{aligned}
& r_{x}=\frac{z_{2}+z_{3}-z_{5}-z_{6}}{2 h \sqrt{3}} \text { and } \\
& r_{y}=\frac{2 z_{1}+z_{2}-z_{3}-2 z_{4}-z_{5}+z_{6}}{6 h} .
\end{aligned}
$$

Equations 4.5 and 4.6 assume a quadratic wavefront between the six actuators surrounding the lenslet. Equations 4.3 through 4.6 are written in matrix form as

$$
\tilde{r}_{s}=\tilde{B} \tilde{z}
$$

where $\tilde{r}_{s}$ is a vector containing the $r_{x}$ and $r_{y}$ slopes and $\tilde{z}$ is a displacement vector. In general, the displacement vector $\tilde{z}$ is a subset of the aberration displacement vector $\tilde{y}$ and is related by

$$
\tilde{z}=\tilde{C} \tilde{y}
$$


Combining Equations 4.7 and 4.8 results in

$$
\tilde{r}_{s}=\tilde{B} \tilde{C} \tilde{y}
$$

and therefore defines the sensor matrix as

$$
\tilde{G}_{s}=\tilde{B} \tilde{C}
$$

Equation (4.9) converts the measured displacements to $\mathrm{x}$ and $\mathrm{y}$-direction average slopes. Note that there are twice as many slopes as lenslets. It should be mentioned that variations to this type of sensor can be implemented, such as weighting additional measured displacement points.

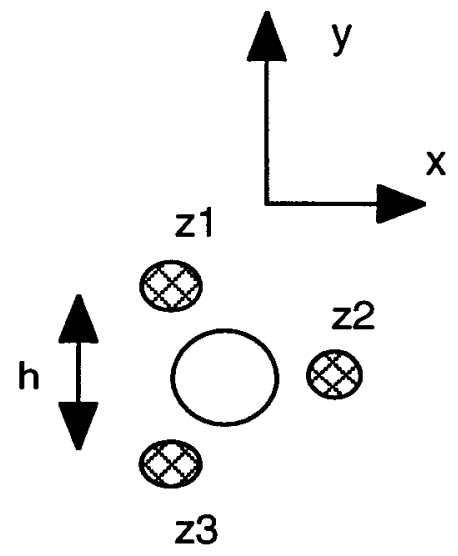

(a)

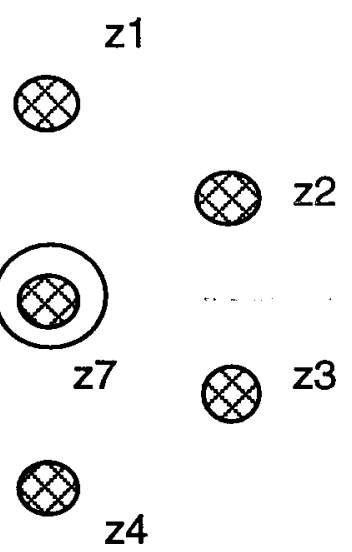

(b)

Figure 4.3 Coordinates and base points used in finite difference equations 


\section{5 - Control Algorithm}

The controller matrix, $\tilde{G}_{c}$, is implemented based on a least squares fit and has been used by many authors (Salmon, et al, 1993, Furber, et al, 1994a, Tyson, 1998) for geometrical or static surface shape control. The controller matrix input output relationship is written as

$$
\tilde{u}=\tilde{G}_{c} \tilde{e},
$$

where the controller matrix, $\tilde{G}_{c}$, is the pseudo inverse of $H$ and is given by

$$
\tilde{G}_{c}=\left(H^{T} H\right)^{-1} H^{T}
$$

and

$$
H=\tilde{G}_{s} \tilde{G}_{p}
$$

Due to $H$ being non-square, the pseudo inverse is required. As discussed earlier, $\tilde{G}_{p}$ is the plant model and utilizes the finite element model developed and validated in Chapter 3, and $\tilde{G}_{s}$ is the Shack-Hartmann sensor model.

\section{6 - Control Simulation}

A Matlab program was written which implements the equations developed in the previous sections and can be found in Appendix C. The National Ignition Facility prototype deformable mirror plant model, developed and validated in Chapter 3, and Shack-Hartmann sensor model were utilized in the simulation. This control model allows different deformable mirror designs and sensor designs to be implemented for a given set 
of wavefront aberrations. Although not presented here, an important aspect of this model is that it allows a comparison between using a perfect sensor, which shows the ability of the deformable mirror to correct the aberration without any sensor filtering, and a sensor which averages over subapertures.

To show the utility of the program some typical cases were considered. Figures 4.4 and 4.7 depicts typical 2 nd order and 4 th order aberrations expected on the National Ignition Facility (NIF). The aberration amplitudes have been reduced to account for the double bounce configuration on NIF. Figures 4.5 and 4.6 and Figures 4.8 and 4.9 show the corrected aberrations for the 2 nd order and 4 th order, respectively. The 2 nd order aberration peak-to-valley was reduced from 4.2 micrometers to 0.25 micrometer, a reduction of $94 \%$. The 4th order aberration peak-to-valley was reduced from 1.05 micrometers to 0.15 micrometers, a reduction of $86 \%$. As discussed in Chapter 3, the model errors are approximately 0.05 micrometers peak-to-valley and represent approximately 20 percent of the 2 nd order and 30 percent of the 4 th order corrected error. This knowledge provides significant value when evaluating performance for design purposes.

The strength and significance of this approach is that it utilizes a verified mathematical model versus less accurate analytical functions as inferred by Salmon, et al (1993) or an unverified model as used by Cielinski (1991). Using a verified model presents the opportunity to complete sensitivity studies or design optimizations with significant confidence without the need to build many expensive prototypes. Compared to Furber, et al (1994a) who developed a correctability model of a large deformable mirror, this approach integrates a Shack-Hartmann sensor model. Lastly, the overall control model shows significant value in simulating static surface shape control problems with the ability to test various deformable mirror, sensor and control algorithm designs. 


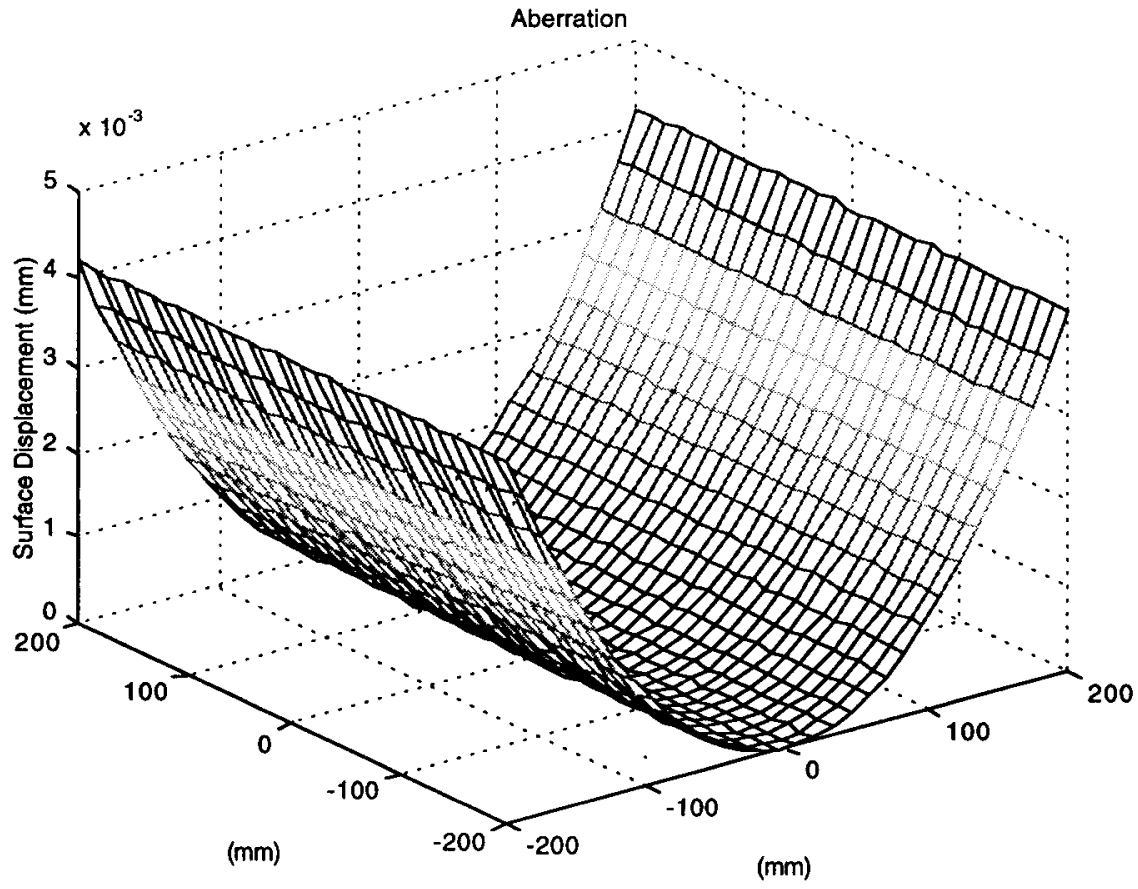

Figure 4.4 Typical 2nd order aberration

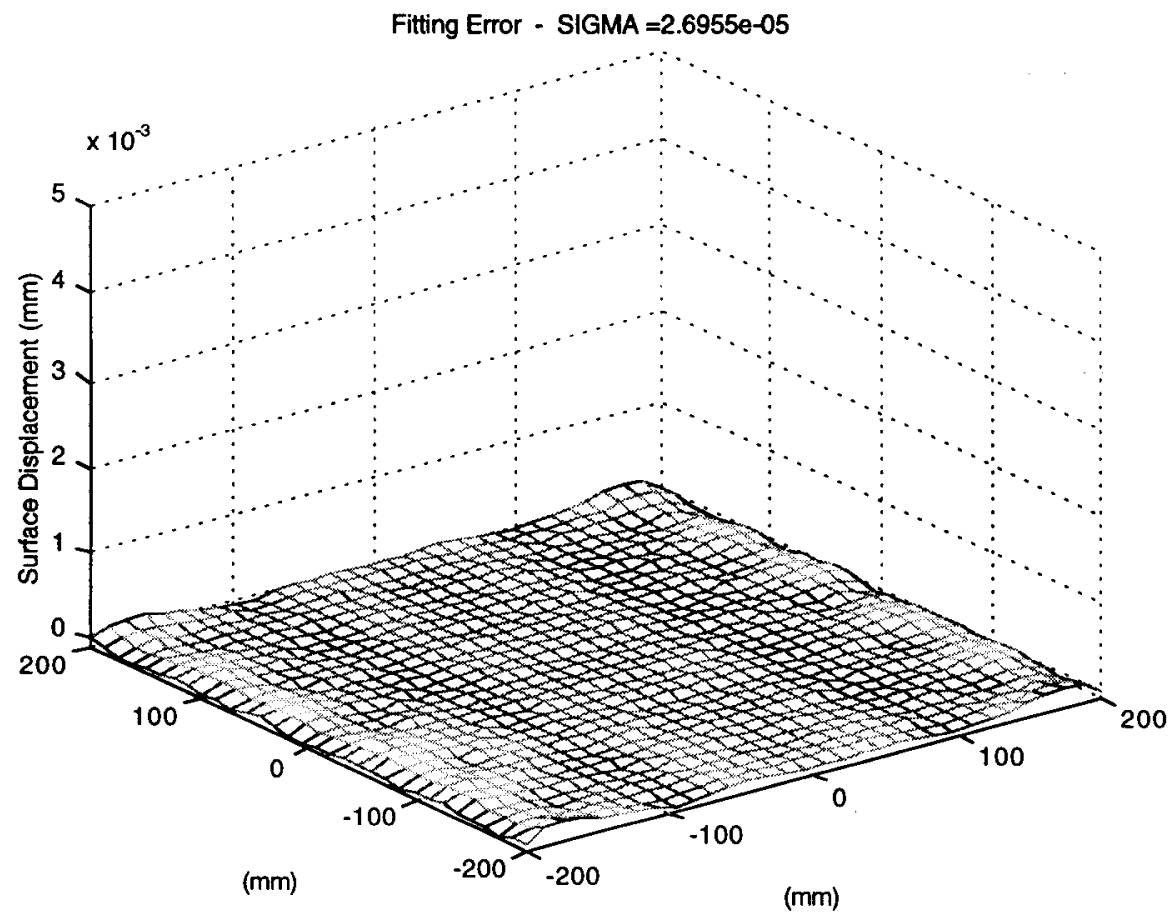

Figure 4.5 Residual after correction of 2 nd order aberration (full scale) 


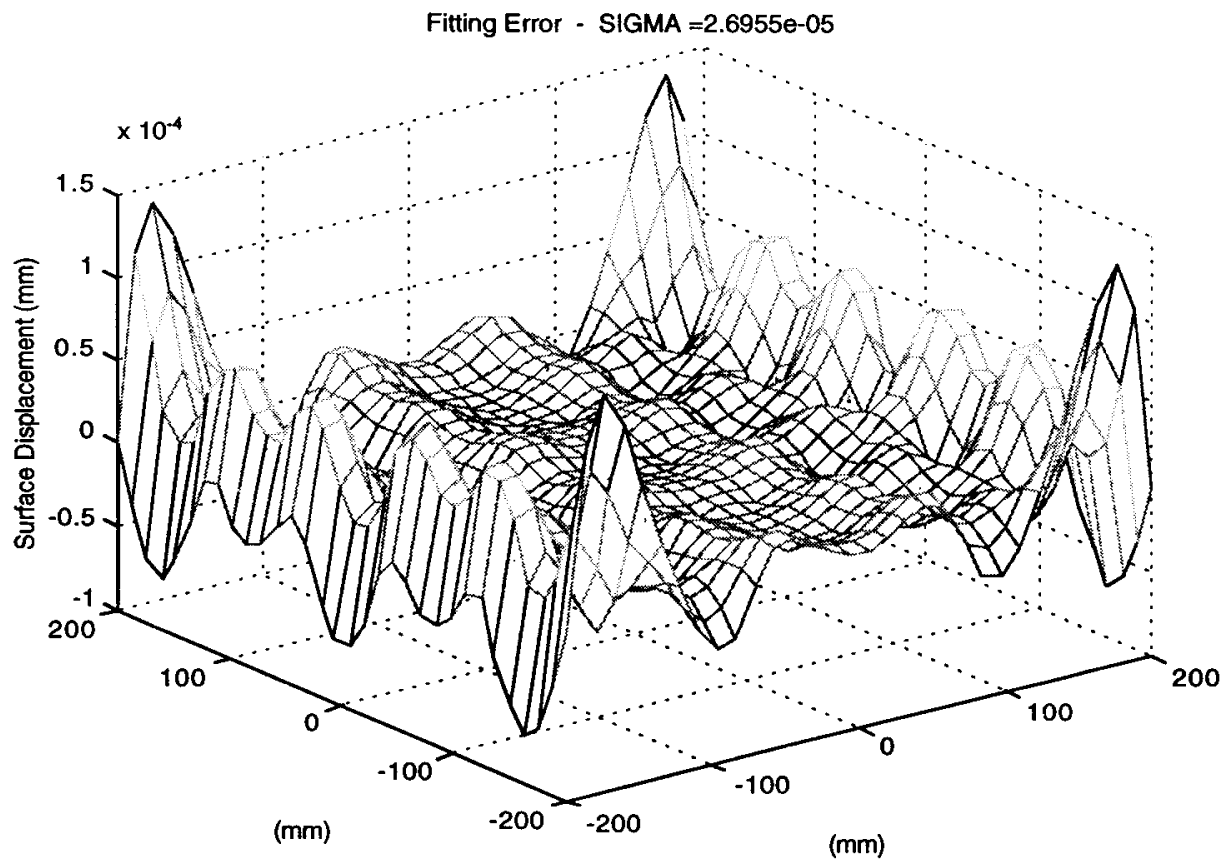

Figure 4.6 Same as Figure 4.5 with different scale

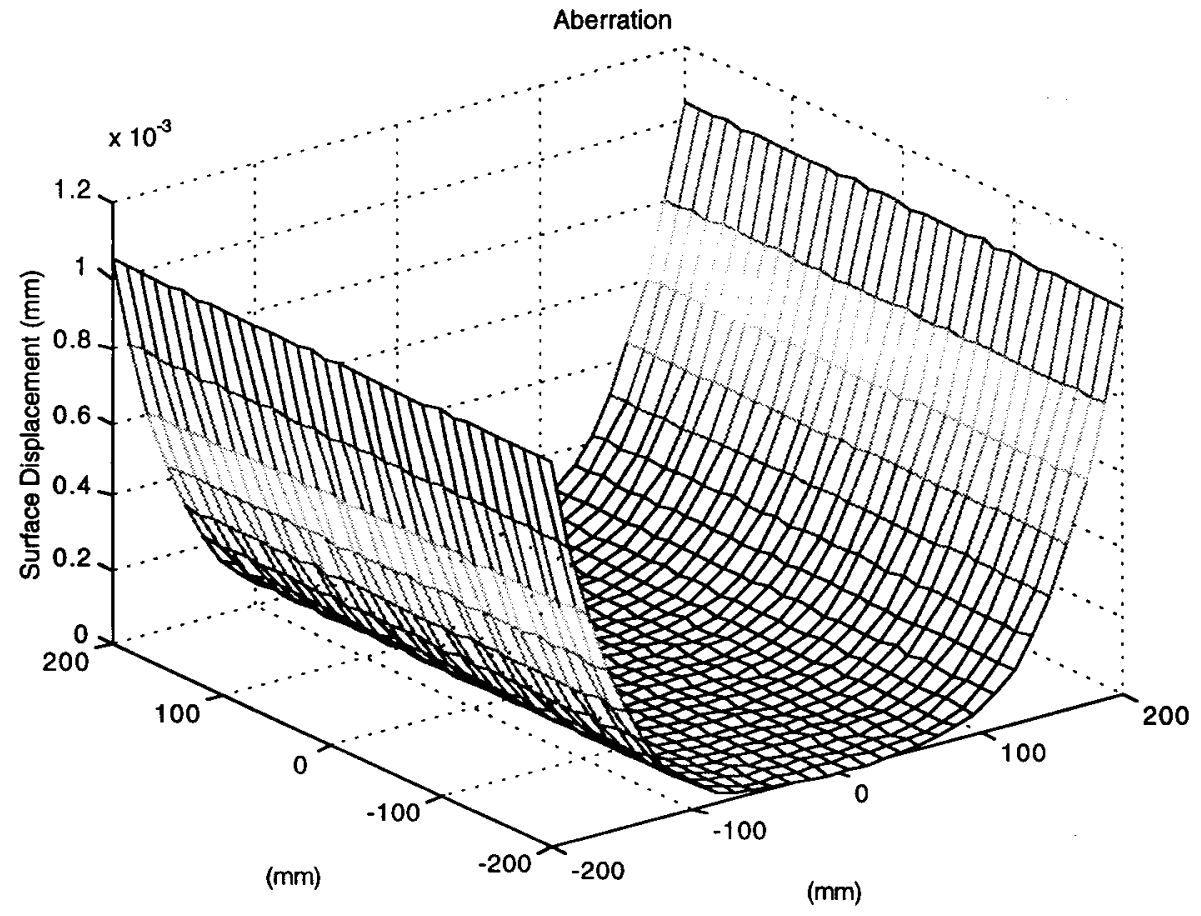

Figure 4.7 Typical 4th order aberration 


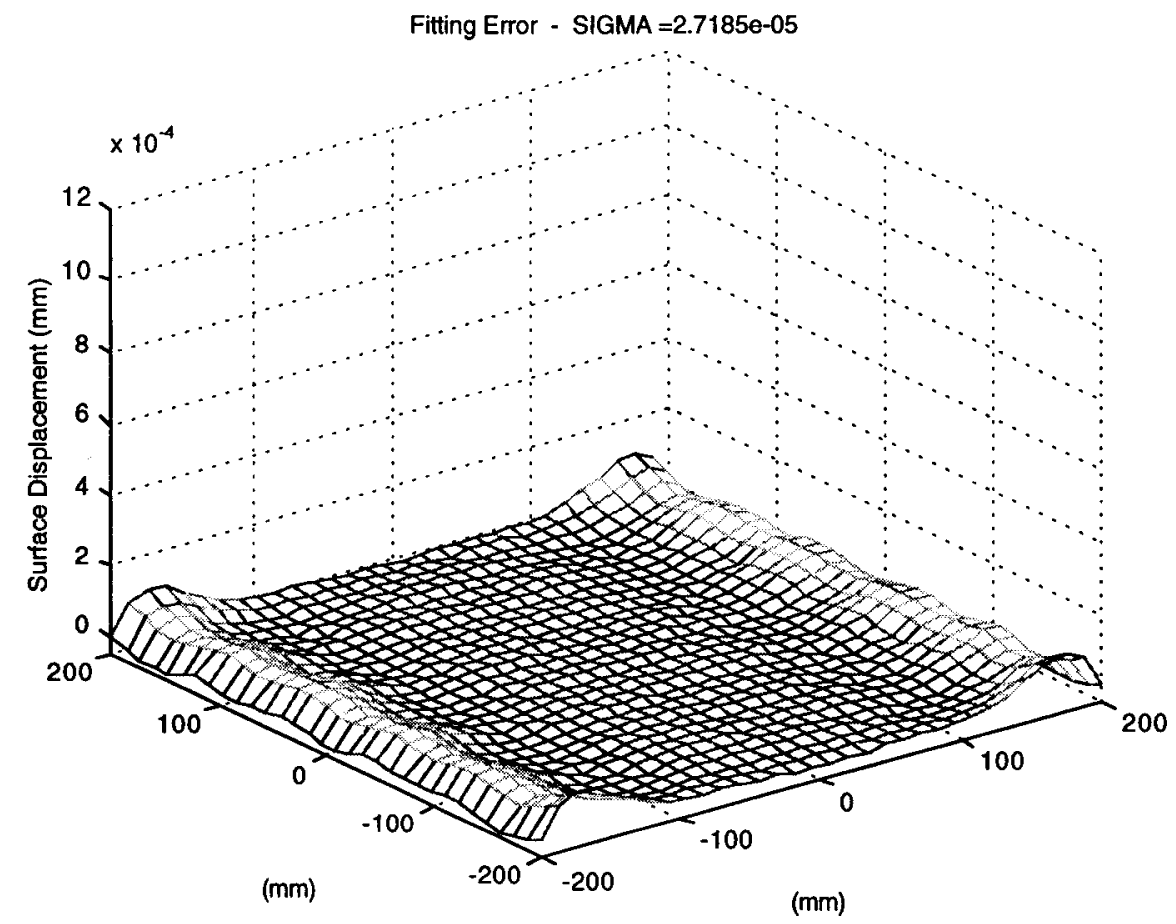

Figure 4.8 Residual after correction of 4 th order aberration (full scale)

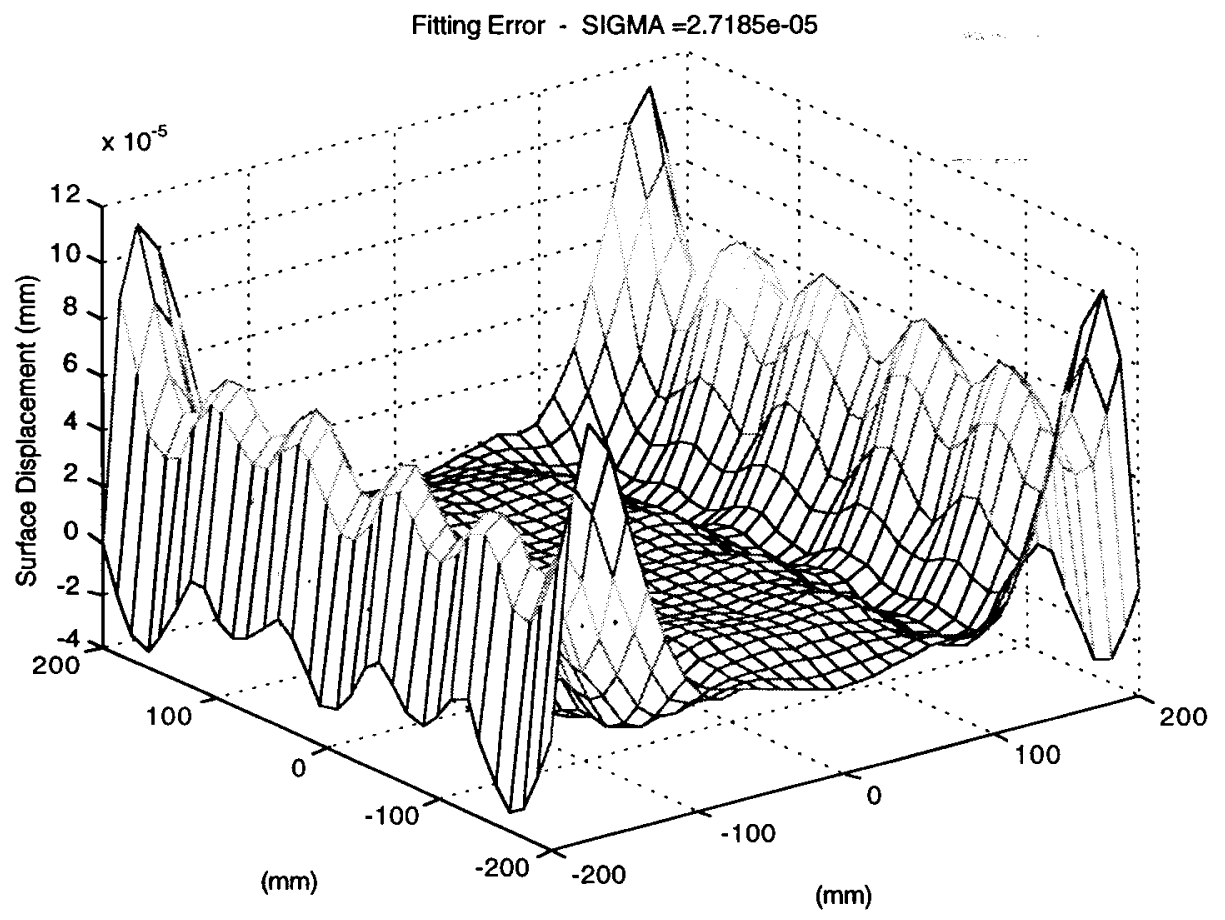

Figure 4.9 Same as Fig. 4.8 with different scale 


\subsection{Performance Comparison}

In this final section, the control model developed was utilized to compare two types of plant models. The errors associated with using traditional analytical functions, e.g., Gaussian functions, versus the validated finite element model developed in this work, was studied. Tyson (1998) presents an analytical influence function representation as

$$
Z(r)=\exp \left(-\beta_{1} r^{2}\right)
$$

where $Z(r)$ is the surface displacement and $\beta_{1}=2.77$. Furthermore, Tyson (1998) states that this simplest Gaussian form represents a deformable mirror influence function quite well, within approximately 5\%. Equation (4.14) was converted to Cartesian coordinates and programmed in Matlab to generate a plant model matrix. This matrix has dimensions of $1024 \times 39$. A simple interpolation technique was used to convert each influence function file to the $32 \times 32$ point grid used previously and depicted in Figure 3.4.

Before generating all 39 influence functions files, influence function \#20 was generated from Equation (4.14) and compared to the experimental data collected in Chapter 3. Using the experimental data, the Cartesian coordinates were adjusted so that Equation (4.14) "matched" the experimental data. These adjusted coordinate values were then used for all 39 influence function files. Figures 4.10 and 4.11 depict influence function \#20 generated by the Gaussian model, and the difference between the model and experiment, respectively. Figures 4.12 and 4.13 depict line plots of the Gaussian and experimental data for actuator \#20. From these Figures, it is evident that the Gaussian function matches the experimental data quite well for actuator \#20 as Tyson (1998) suggests.

With the Gaussian plant model developed, three disturbance cases were solved and compared to the finite element model solution. The three cases included, a piston aberration, a 2nd order aberration, and a 4th order aberration. The piston aberration is 
probably the most important test case, since the answer is known, i.e., the resulting surface should be perfectly flat.

The first comparison was made with the piston aberration correction. The Gaussian plant model produces residual errors approximately 29 times greater than the finite element model results. Figures 4.14 and 4.15 depict the errors associated with the Gaussian and finite element model results, respectively. As expected, the Gaussian function produced large errors near the edges, since these analytical functions do not maintain continuity in displacement, slope, and higher derivatives between adjacent influence functions or at edge boundary conditions. Authors (Tyson, 1998, Sacks, 1998b) have implemented techniques or "tricks" to achieve better boundary condition matches using analytical functions. Figures 4.17 and 4.19 depict the correction errors for the 2nd order and 4th order aberrations, respectively, for the Gaussian function. These results compare to Figures 4.5 and 4.8 which utilized the finite element plant model. The Gaussian plant model 2 nd order residual error is approximately 6 times greater than the finite element results, and the 4 th order residual error is approximately 2 times greater. These results are summarized in Table 4.1.

\begin{tabular}{|c|c|c|c|c|c|c|}
\hline Aberration & Form & $\begin{array}{c}\text { P-V } \\
\text { displacement } \\
(\mu \mathrm{m})\end{array}$ & $\begin{array}{c}\text { Gaussian } \\
\text { residual } \\
(\mathrm{rms})(\mathrm{nm})\end{array}$ & $\begin{array}{c}\text { Finite Element } \\
\text { residual (rms) } \\
(\mathrm{nm})\end{array}$ & $\begin{array}{c}\text { Percent } \\
\text { Difference } \\
(\%)\end{array}$ & $\begin{array}{c}\text { Multiple } \\
\text { Factor }\end{array}$ \\
\hline Piston & $\mathrm{a}$ & 1 & 40.1 & 1.40 & 2800 & 29 \\
\hline 2nd order & $\mathrm{by}^{2}$ & 4 & 150 & 26.9 & 457 & 5.6 \\
\hline 4th order & $\mathrm{cy}^{4}$ & 1 & 65.1 & 27.2 & 140 & 2.4 \\
\hline
\end{tabular}

Table 4.1 Comparison between Gaussian and finite element plant models 


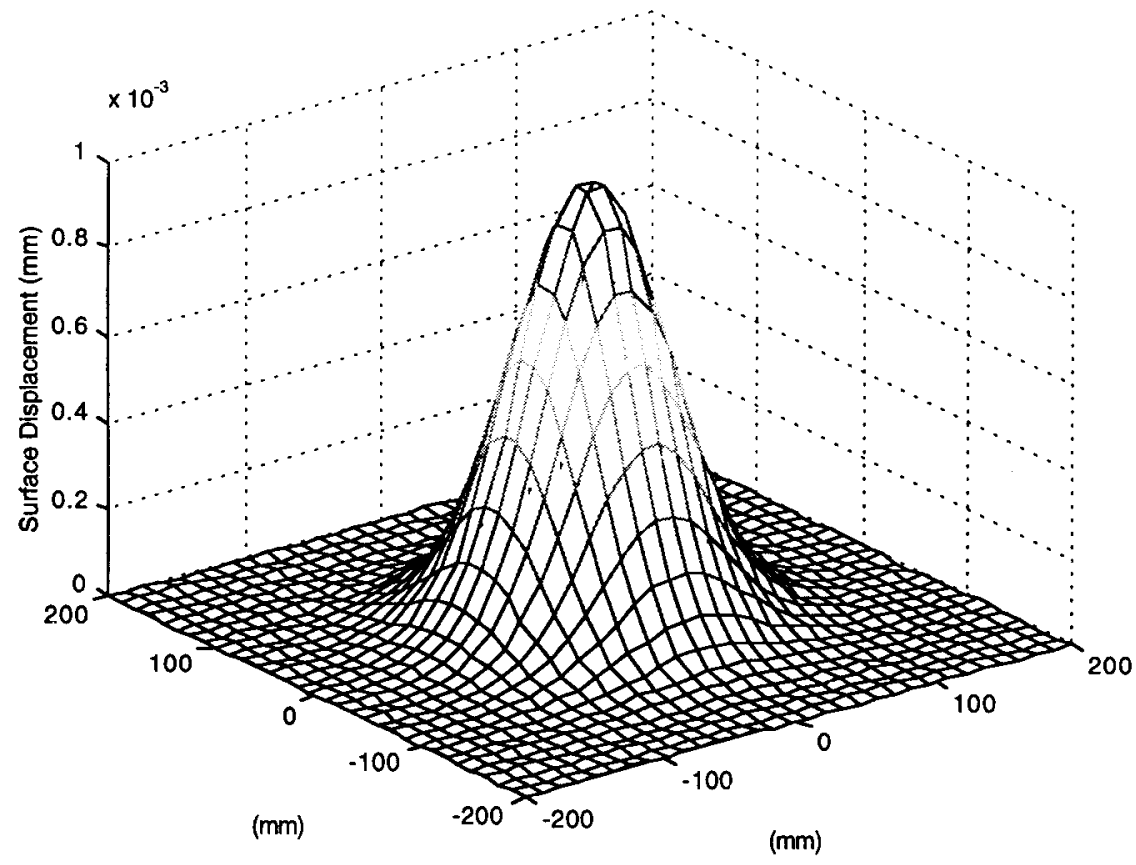

Figure 4.10 Normalized Gaussian influence function using Equation (4.14)

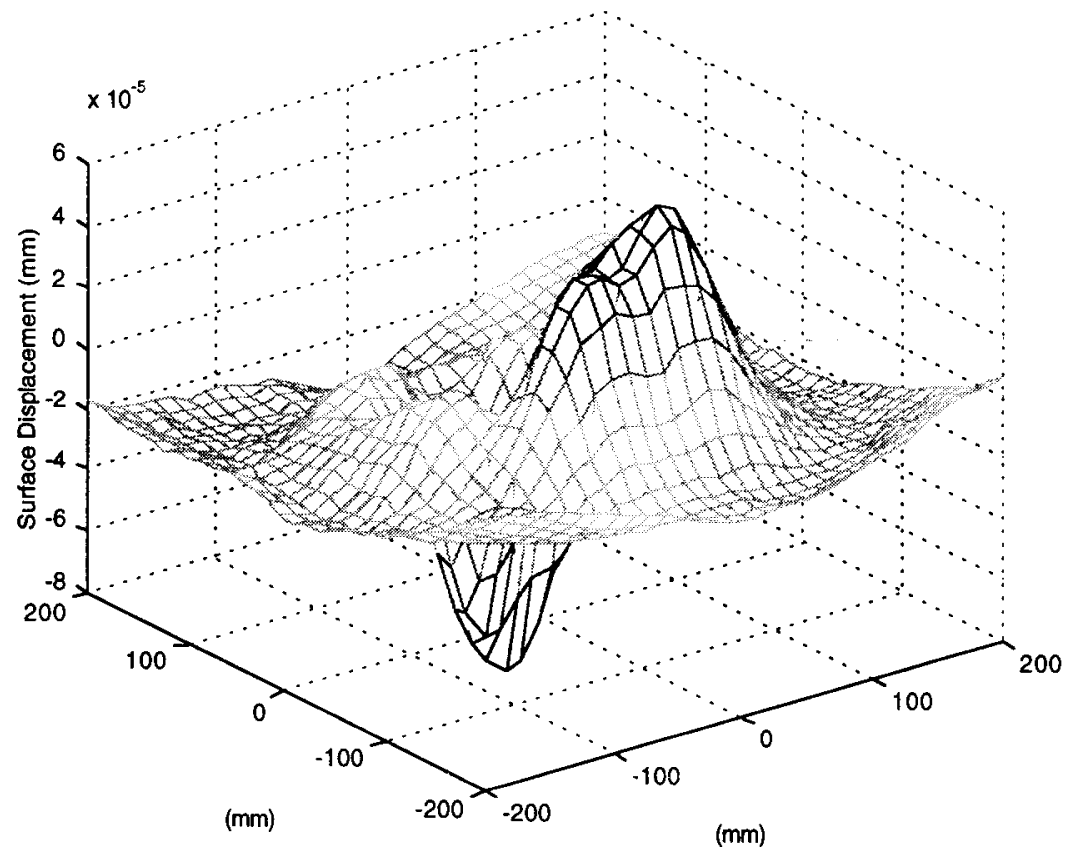

Figure 4.11 Difference between Gaussian and experimental results 


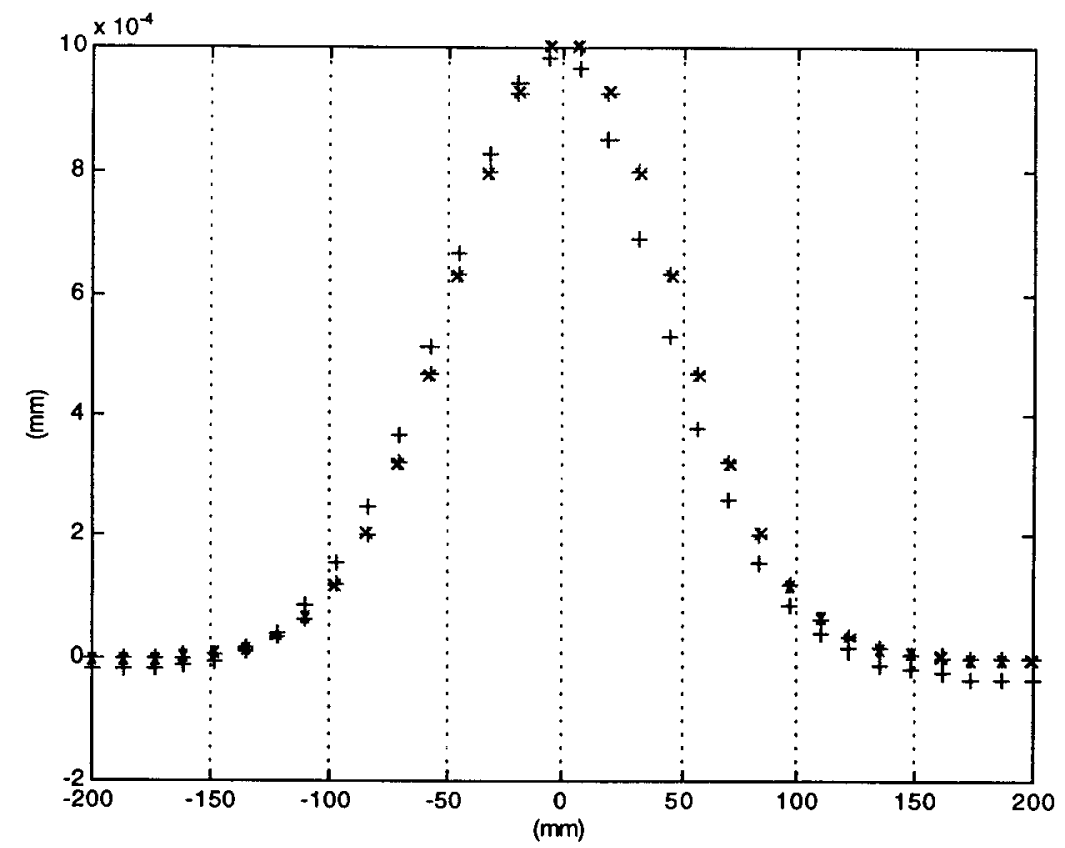

Figure 4.12 Line plot of Gaussian model (*) and experiment (+) for act. \#20 (y-direction)

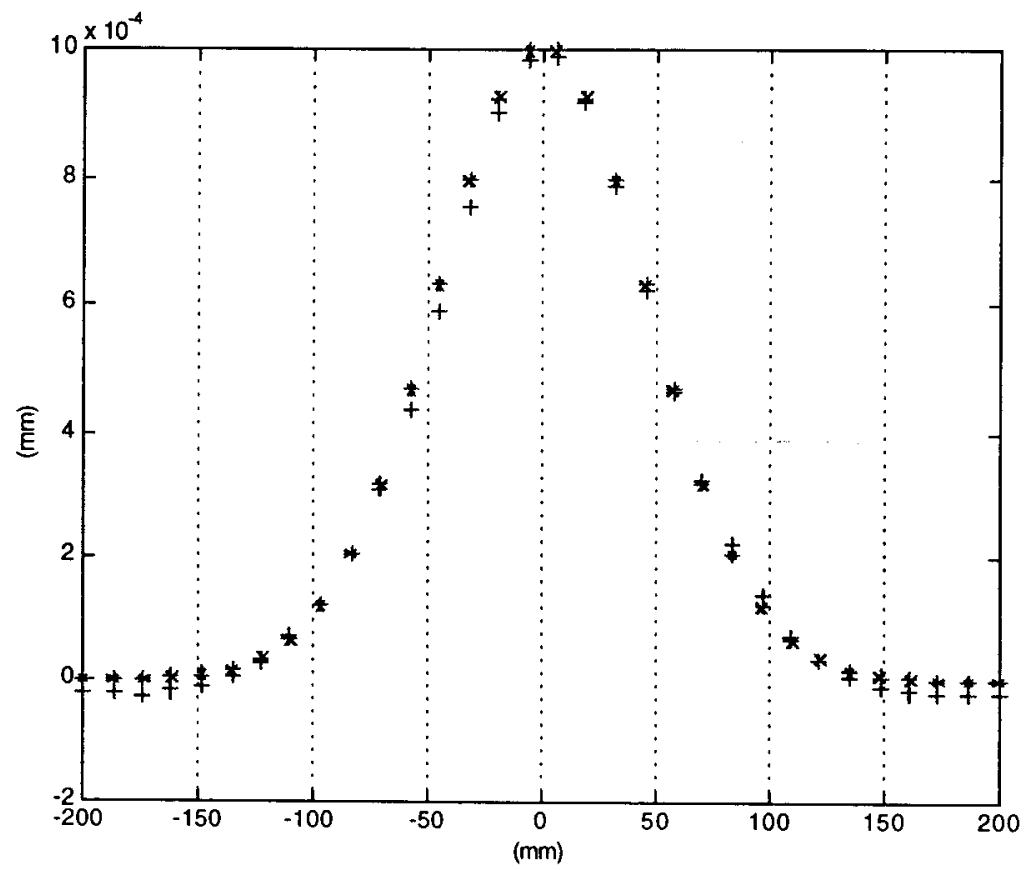

Figure 4.13 Line plot of Gaussian model $(*)$ and experiment $(+)$ for act. \#20 (x-direction) 


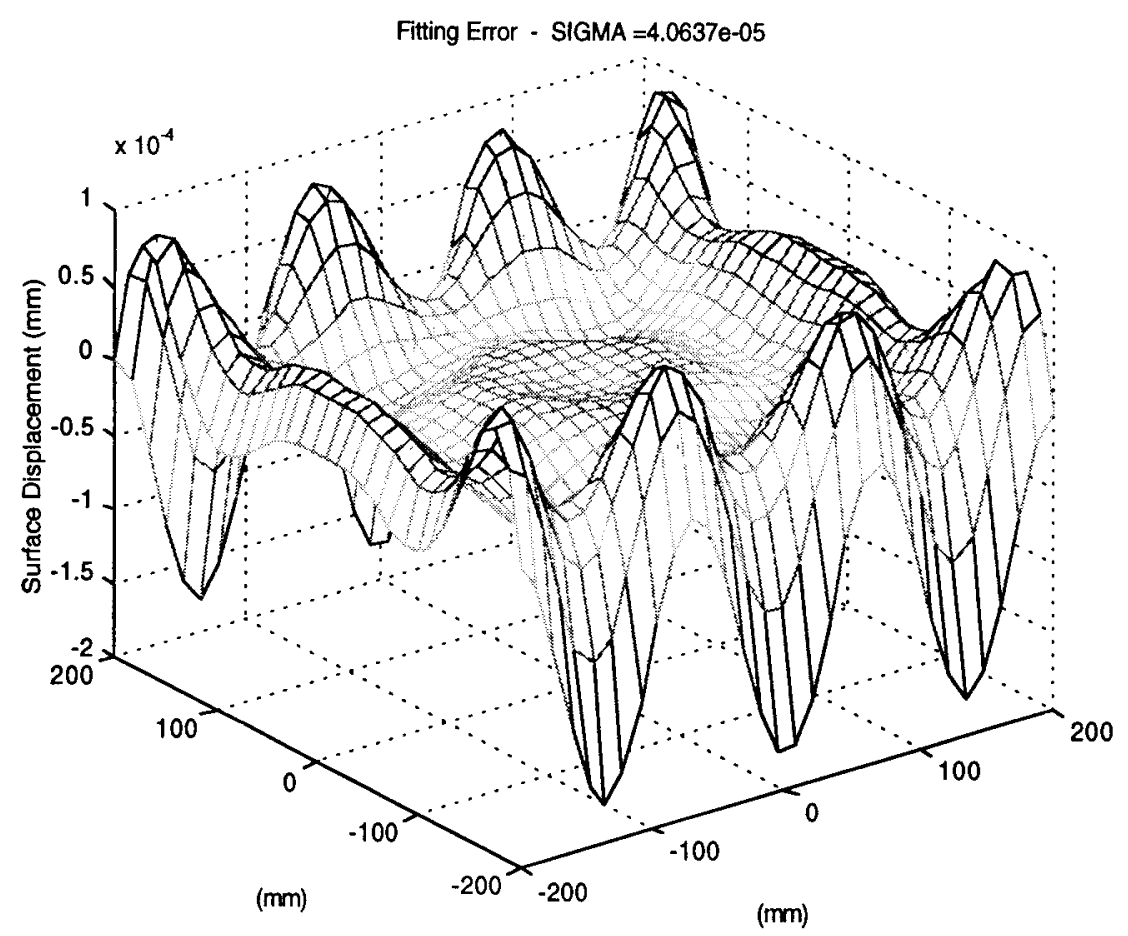

Figure 4.14 Correction of a piston aberration with Gaussian model

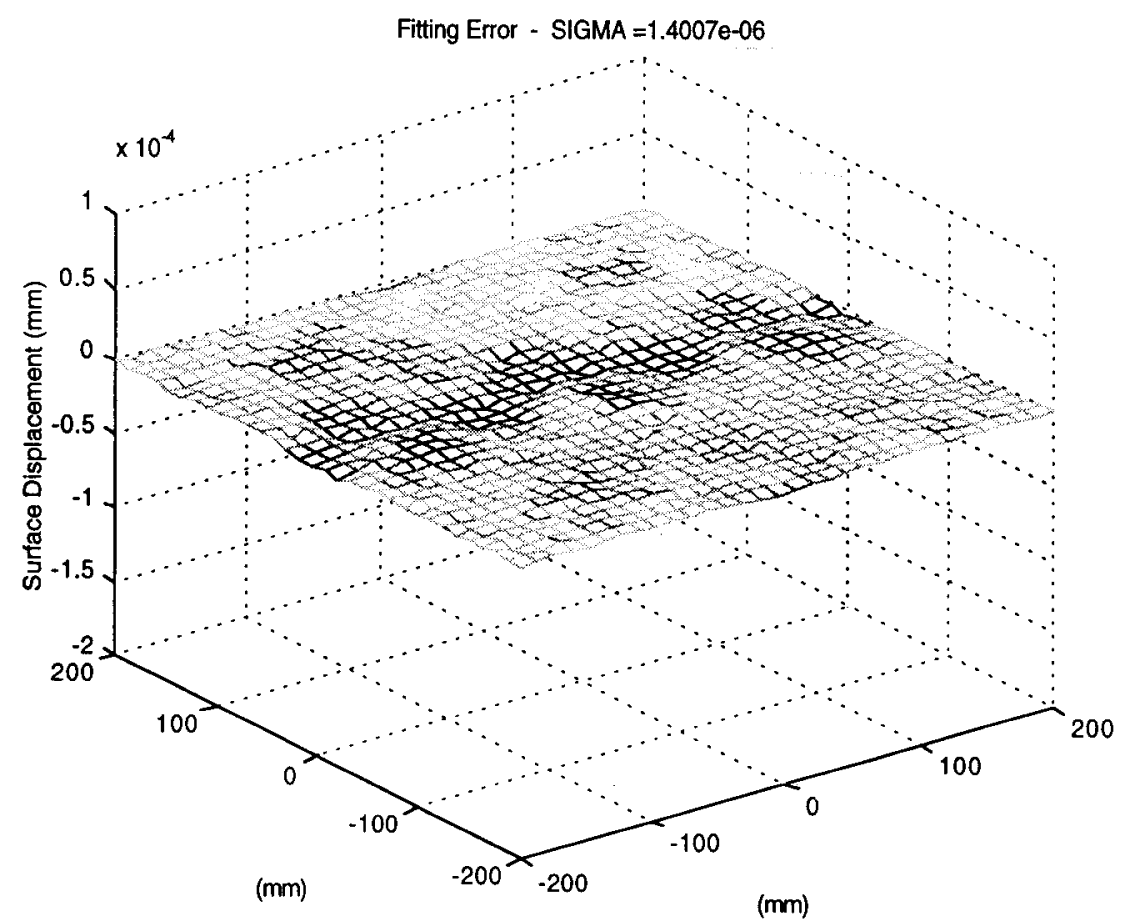

Figure 4.15 Correction of a piston aberration with FE model (same scale as Fig. 4.14) 


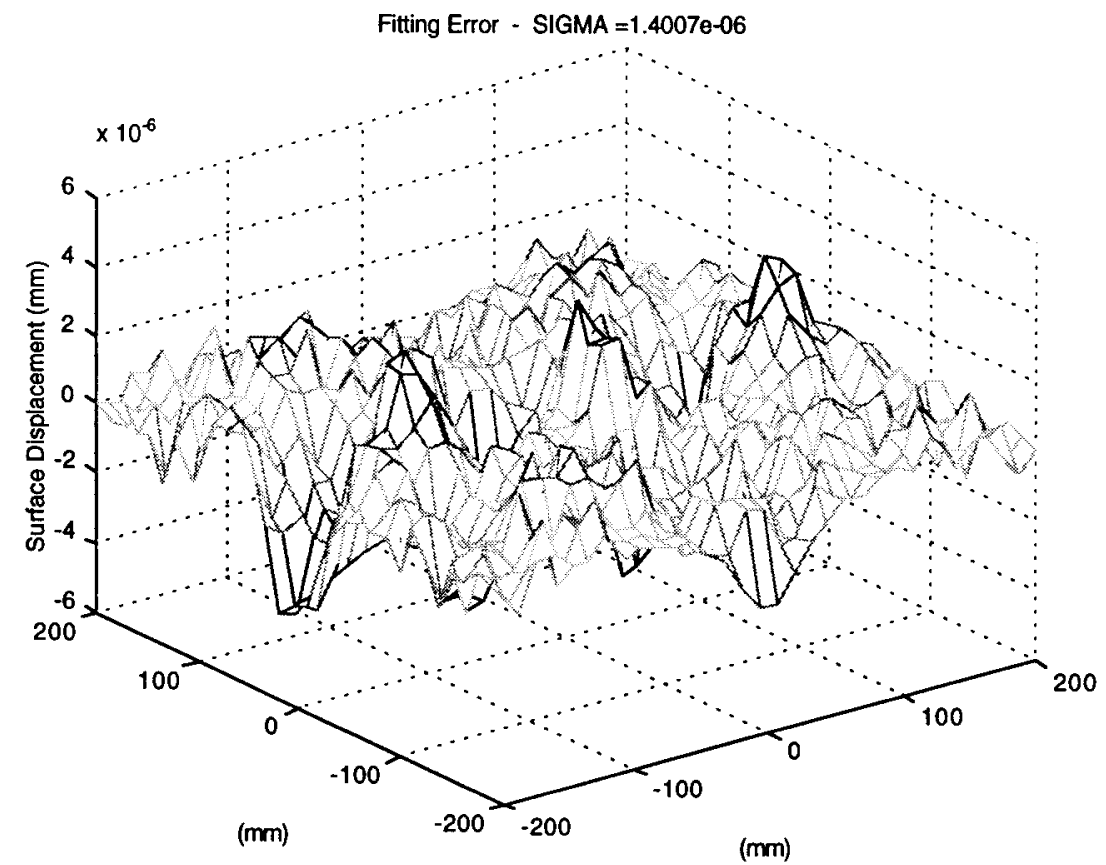

Figure 4.16 Same as Fig. 4.15 with different scale

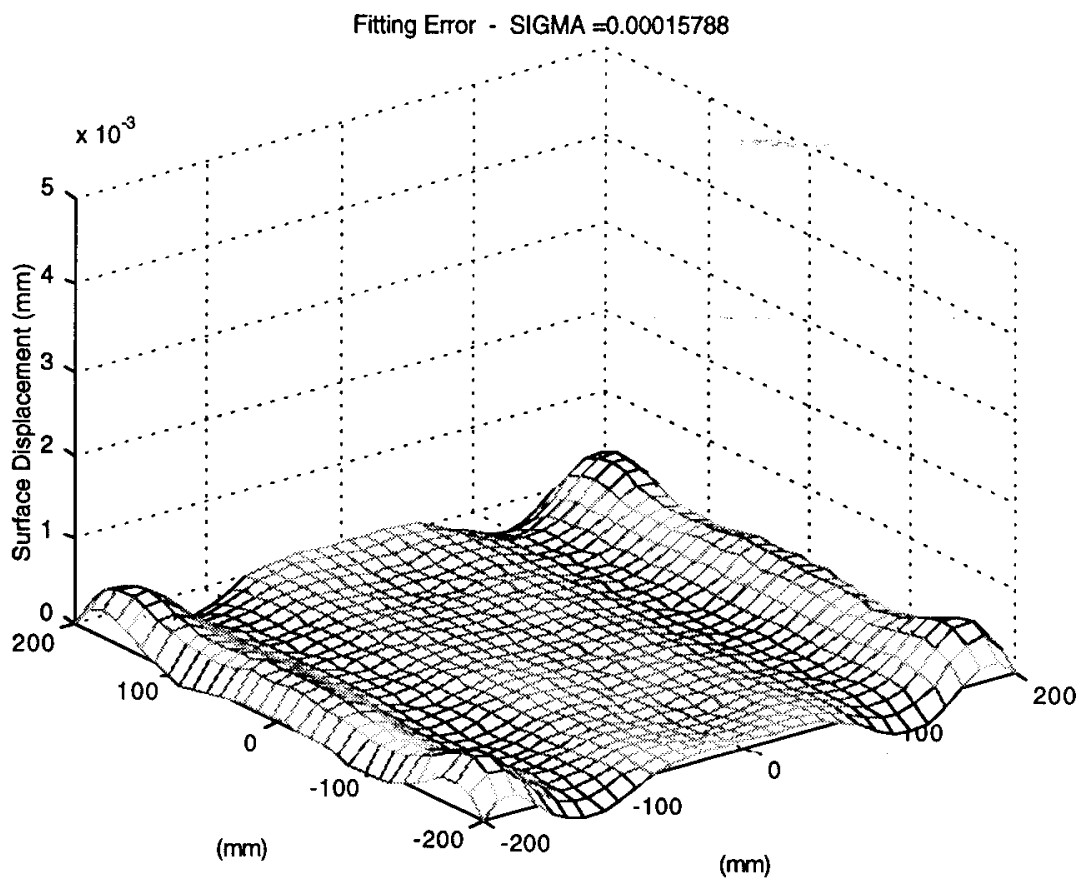

Figure 4.17 Residual after correction of 2nd order using Gaussian model 


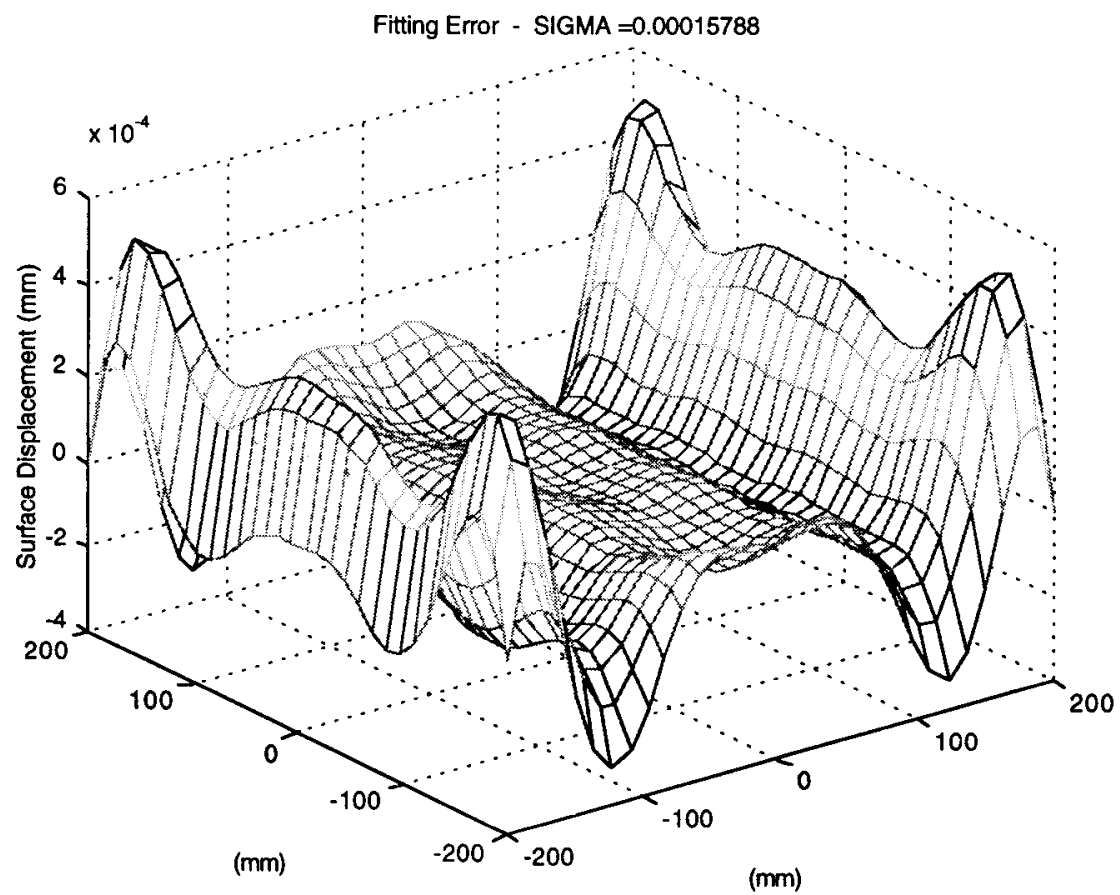

Figure 4.18 Same as Fig. 4.17 with different scale

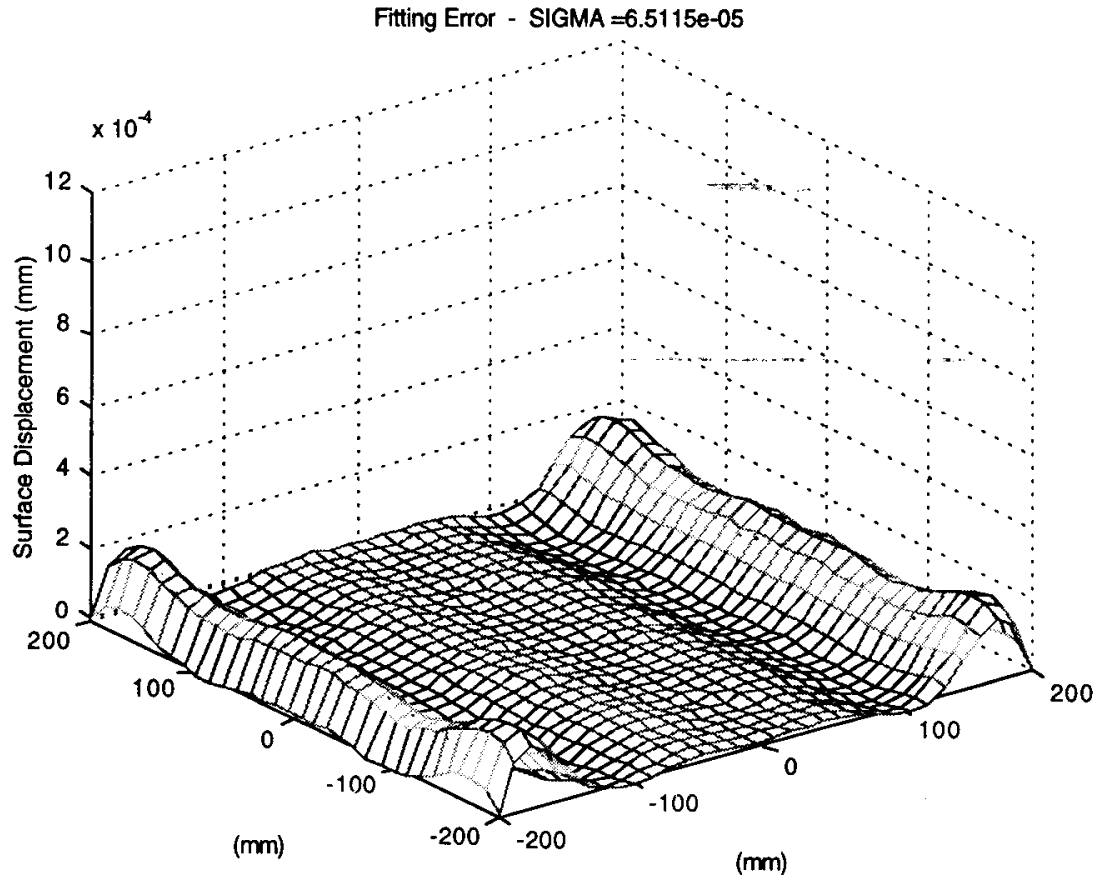

Figure 4.19 Residual after correction of 4th order aberration with Gaussian model 


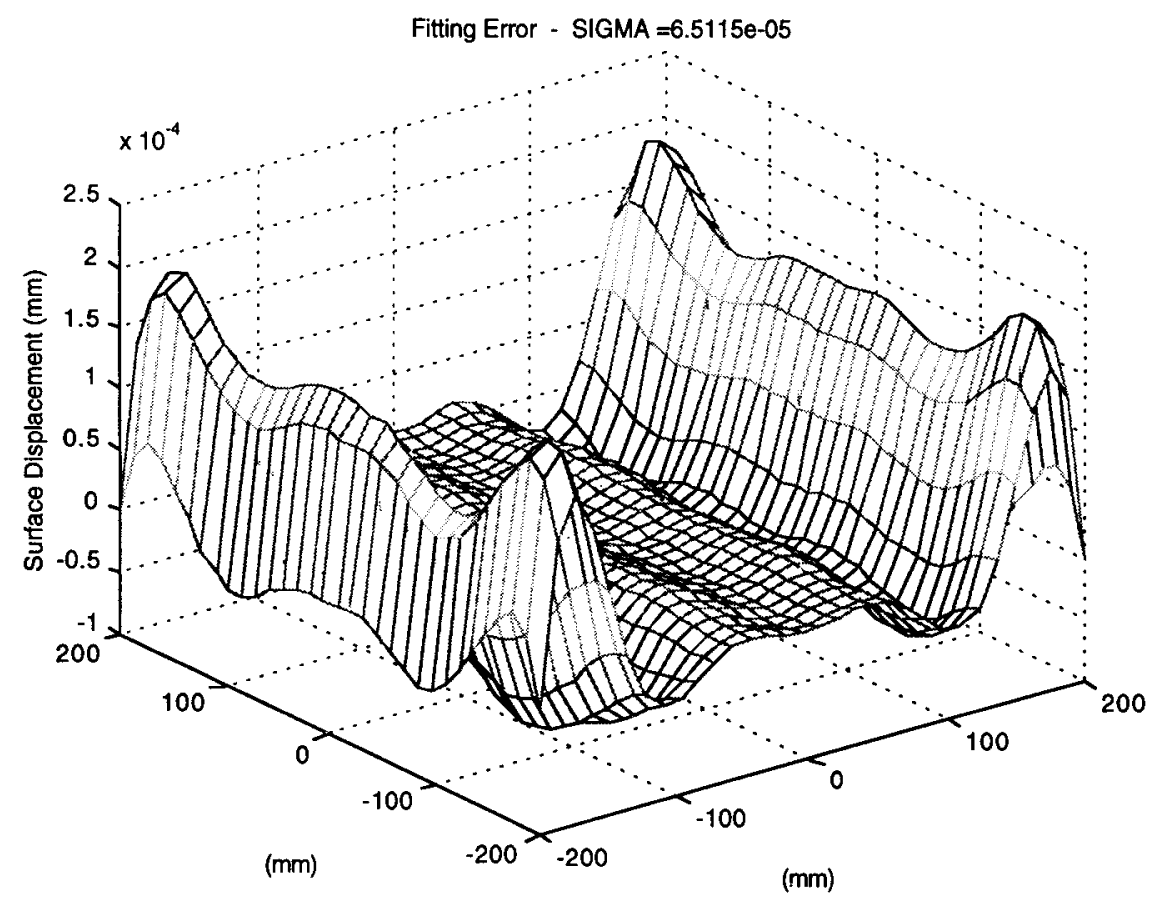

Figure 4.20 Same as Fig. 4.19 with different scale

\section{8 - Conclusion}

In this chapter, static surface shape control was investigated and implemented using the NIF prototype deformable mirror and sensor configuration. The plant matrix utilized the finite element model developed and validated in Chapter 3. The sensor matrix was based on a Shack-Hartmann configuration and the controller algorithm was based on a least squares method. Equations were coded using Matlab and simulations were completed for typical NIF aberrations. This control model shows significant value in simulating static surface shape control problems with the ability to test various deformable mirror, sensor and control algorithm designs. Lastly, the control model was utilized to compare the residual errors of a typical Gaussian function model versus the validated finite element plant model. As expected, since the Gaussian function does not maintain continuity in displacement, slope and higher derivatives between adjacent influence functions and at edge 
boundary conditions, the residual errors from the Gaussian model exceeded the errors of the finite element model. Depending on the aberration corrected, the Gaussian plant model produced residual errors 2 to 29 times greater than the validated finite element plant model. 


\section{CHAPTER 5 - DYNAMIC SURFACE SHAPE MODELING}

\subsection{Introduction}

The purpose of this chapter is to develop a computer model which will facilitate the development of dynamic surface shape control approaches. This model will be used in the next chapter to develop proportional-integral (PI) and $\mathrm{H}_{\infty}$ control algorithms. The first part of this chapter will cover the general model development approach and related research. The second part will address model reduction techniques. The third section will cover derivation of the dynamical equations of motion utilizing bond graph techniques, for both SISO and MIMO models. Lastly, the final section will cover verification of the dynamic equations of motion, utilizing experimental results from the NIF prototype deformable mirror and published literature results.

\subsection{Model Development}

This phase of research has the ultimate goal of developing a dynamic model of a uniform, homogenous, isotropic continuous plate with discrete linear actuators, such as the one shown in Figure 5.1. Such a model can be considered to represent a deformable mirror. The general partial differential equation for this system can be written as (Meirovitch, 1967)

$$
\begin{gathered}
D\left(\frac{\partial^{4} w}{\partial x^{4}}+\frac{2 \partial^{4} w}{\partial x^{2} \partial y^{2}}+\frac{\partial^{4} w}{\partial y^{4}}\right)+\rho \frac{\partial^{2} w}{\partial t^{2}}=f(x, y, t) \\
D=\frac{E h^{3}}{12\left(1-v^{2}\right)}
\end{gathered}
$$

where

$E=$ Young's Modulus,

$h=$ plate thickness,

$v=$ Poisson's ratio, 
$\rho=$ mass/unit area, and

$f(x, y, t)=$ actuator dynamics.
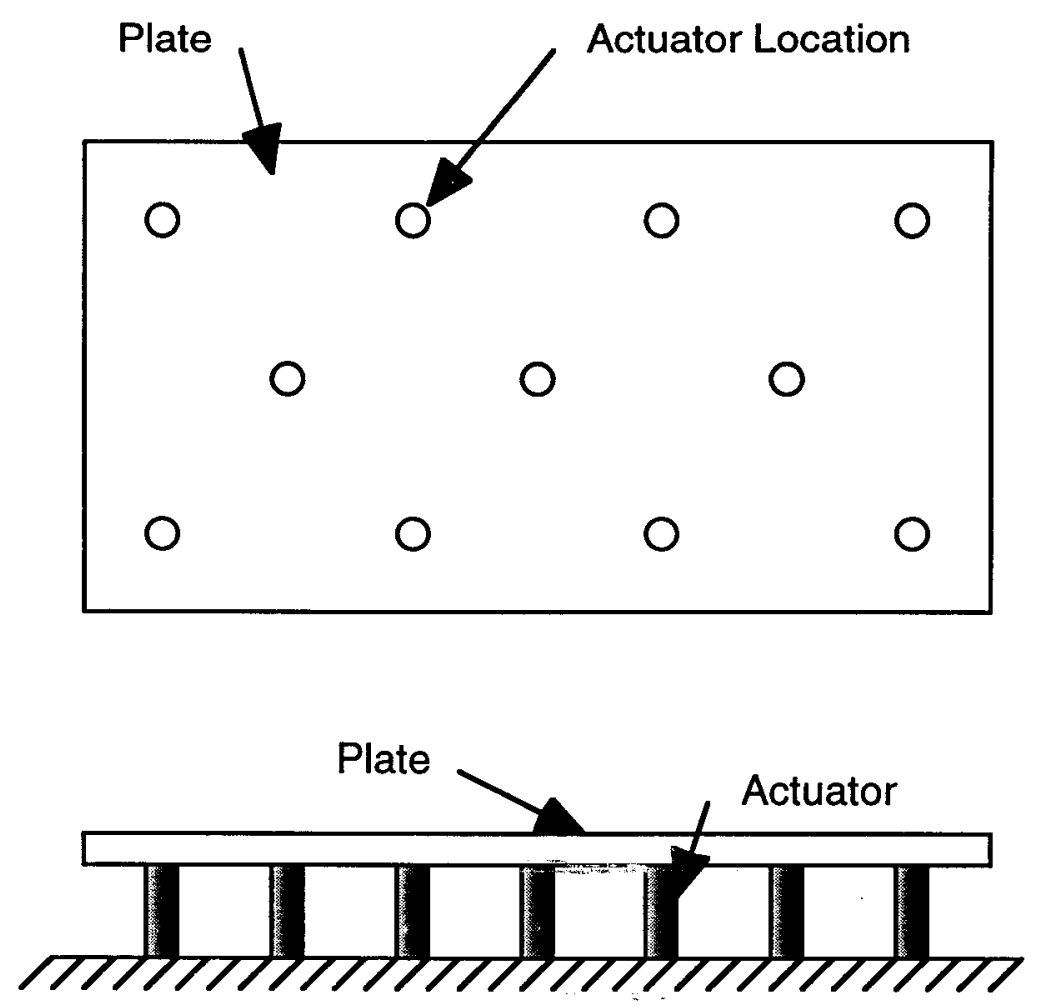

Figure 5.1 Plate and actuator layout

This model has been used many times (Creedon and Lindgren, 1970; Walker and Yaneskek, 1976b; Apollonov, et al, 1990; Blanton and Sadek, 1992) in modeling plate systems. However, most authors use a simplified force input instead of the actual actuator dynamics. The reason for this is that using known simple force inputs allows a set of uncoupled differential equations to be developed, whereas using the actual actuator dynamics leads to a set of equations which are no longer uncoupled and cannot be solved independently. When adding the actuator dynamics, these models are referred to as combined dynamical models since they are a combination of discrete and continuous elements (Nicholson and Bergman, 1986). The approach of this work differs from 
published modeling approaches (Marlow, 1994; Boyer, et al, 1991; Vincent, et al, 1990; Freeman and Garcia, 1982) in that it incorporates dynamic actuator coupling and plate compliances in a combined dynamical system model. The results of an intensive literature review have shown that this approach has not been previously applied to adaptive optics systems.

The difficulty in developing mathematical models for this type of system is that models typically become extremely large and unusable for controller development. The next section will cover various model reduction techniques to address this shortcoming.

\subsection{Model Reduction}

Model reduction is a very important aspect to consider when developing models for control purposes. A significant amount of research has been completed related to model reduction techniques, especially in the large space structure field. Three in-depth literature reviews covering model reduction can be found in Junkins (1990), Meirovitch (1990) and Fortuna, et al (1992).

Using the state space representation, the following relationships can be developed which help describe the model reduction problem. The primary objective is to obtain a low order model such that the output vector $y_{r}$ best approximates, in accordance with some criteria, the output $y$ for all the signal vectors $u$ belonging to the class of admissible input functions. First, the original model is described as a high order time invariant system as

$$
\begin{aligned}
& \dot{x}=A x+B u \\
& y=C x
\end{aligned}
$$

where,

$$
x \in R^{n}, y \in R^{p}, u \in R^{m} .
$$

Next, a low-order model of the original is assumed to have the form

$$
\begin{aligned}
& \dot{x}_{r}=A_{r} x_{r}+B_{r} u \\
& y_{r}=C_{r} x_{r}
\end{aligned}
$$


where,

$$
x_{r} \in R^{r}, y_{r} \in R^{p}, r<n \text {. }
$$

The purpose of model reduction is to reduce the number of states a model possesses in order to be tractable for realistic controller design. In general, during this reduction technique, dynamic characteristics of the model will be lost. Therefore, the primary goal of model reduction will be to minimize the loss of useful information. An important question asked by Safonov (1991) is "How accurate must a reduced model be in order to be reliably used?". As discussed by Fortuna, et al (1992), there exists no universal model reduction scheme which can be applied, and in general, a combination of approaches gives the best results. The first type of classification can be given by referring to the domain where the models are represented; i.e., either frequency or time. Another type of classification has been provided by Skelton (1980), who suggests three categories of model reduction procedures:

1. Methods based on polynomial appitoximations (usually suitable in the frequency domain),

2. Component truncation procedures based on state-space transformations, and

3. Parametric optimization techniques.

The purpose of this next section is to review many of the most used model reduction approaches, which will primarily focus on the state truncation techniques. It should be pointed out that other reduction approaches exist, such as controller reduction. However, these approaches will not be addressed here.

The Aggregation method, introduced by Aoki (1978), is based on the concept of combining a defined set of state variables of the ofiginal system with chosen weighting factors. The perfect aggregation problem consists of finding a matrix that relates the original state space to the reduced order state space. This matrix is called the aggregation matrix. The adequacy of the reduced state vector, and therefore the aggregation matrix, 
depends on the objective desired; open-loop or closed-loop performance, optimization of certain indexes, etc. (Fortuna, et al, 1992). The Singular Perturbation-based Approach proposed by Kokotovic, et al (1986) is applicable to systems that can be heuristically decoupled a priori into two subsystems, one slow and one fast. As a first approximation, the slow part of the system can be considered dominant for a description of the full behavior, and therefore, the reduced order model (Fortuna, et al, 1992). The Error Minimization Approach proposed by Wilson and Mishra (1979) defines a cost function which one tries to minimize. The cost function contains a weighting positive-definite symmetric matrix and a vector which is defined as the error between the outputs of the original system and those of the reduced-order system (Fortuna, et al, 1992).

The Modal Cost Analysis Approach defines a cost for the open loop model. In general, the cost of each state is uniquely defined, and through optimization, the states with the highest cost are retained (Skelton, et al, 1982). A Multivariable Frequency-Weighted Approach is proposed which focuses on "cross-over" or midrange frequencies. Due to the fact that at low- and high-frequencies an extremely-accurate model is not required, model reduction can focus on preserving the accurate midrange frequencies. This reduction approach assumes the state-space model can be decomposed into two components. It is desirable that one component dominates the important characteristics of the system to be modeled, whereas the other is less significant (Bacon, et al, 1989).

The Internally Balancing Theory Approach was proposed by Gregory (1984). This approach produces a balanced approximation to the large model by defining and retaining the most controllable and observable states. Although practically very difficult to implement, approximations have been developed for MIMO lightly damped structures. Mode selection requires evaluation of the relative impoitance of each mode of the control problem: 1) modal fidelity, 2) controllability, 3) observability, 4) disturbance environment, and 5) performance objective (Gregory, 1984). Hankel based methods propose the problem of model reduction is to find a transfer matrix, with a degree less than the original 
system, such that the Hankel norm of the error matrix will be minimized. The error matrix is defined as the original system matrix minus the transfer matrix (Fortuna, et al., 1992).

As discussed above, the purpose of model reduction is to reduce the number of states a model possesses in order to be tractable for realistic controller design. Accordingly, the primary goal of model reduction will be to minimize the loss of useful information. Although the Aggregation and Singular Perturbation approaches are appealing for this system configuration, the reduction approach employed will utilize a priori information about the system to minimize the number of states.

Figure 5.2 depicts a general modal density plot of an actuator/plate system which is representative of a deformable mirror. If system input frequencies are known, by examining the modal density plot of the system, selected states may be eliminated from the model since they will not contribute significantly. This technique is based partially on engineering judgment (Karnopp, et al, 1990) and will be referred to as the modal density selection approach. Region I has no modes and is therefore a compliance or static based system. Many adaptive optic systems are developed utilizing this type of model. Region II is defined as including the compliance components and the first dynamic mode of the system. Generally, this mode is derived from actuator interactions. Region III is defined as the entire system model including compliance components, actuator dynamics and plate modes. Utilizing this modal density selection approach, the equations of motion will be developed in the next section. 


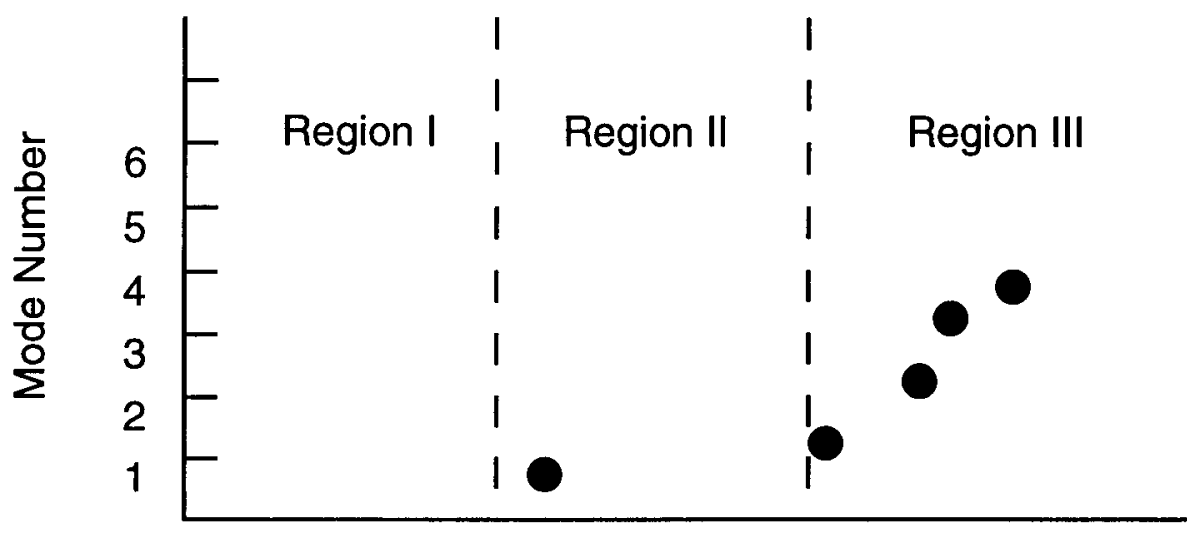

Frequency

Figure 5.2 Modal density plot for actuator/plate system

\subsection{Derivation of Dynamical Equations of Motion}

Bond graphs are used to develop the dynamical equations of motion for a single actuator system and a seven actuator plate system. Bond graphs were chosen for their concise, pictorial representation of energy storage, dissipation and exchange mechanisms of interacting dynamics systems, subsystems and components. Bond graphs utilize a picture in a manner analogous to circuit diagrams, freebody diagrams, block diagrams or signal flow diagrams. However, bond graphs are more concise and facilitate selection of state variables and derivation of first order differential equations which are easily converted to state space form (Margolis, 1985). Next, the derivation of the equation of motion using bond graph techniques will be outlined.

\section{Nomenclature}

The nomenclature listed below, describes the variables used in deriving the equations of motion, and are expressed as

$$
\begin{aligned}
& q_{i}=\text { Displacement variable, } \\
& e_{i}=\text { Effort variable, }
\end{aligned}
$$




$$
\begin{aligned}
& \dot{q}_{i}=f=\text { Flow variable, } \\
& \dot{e}_{i}=p=\text { Momentum variable }, \\
& R_{i}=\text { Resistance value } \\
& C_{i}=\text { Compliance value } \\
& I_{i}=\text { Inertia value. }
\end{aligned}
$$

Following the techniques developed by Karnopp, et al (1990), the independent state variables are identified directly from the bond graph as a generalized momentum variables, $p^{\prime} s$, on all inertia $(I)$ elements and generalized displacement variables, $q$ 's, associated with the compliance $(C)$ elements where

$$
p=\int_{0}^{t} e d t
$$

and

$$
q=\int_{0}^{t} f d t
$$

and $e, f$ are the respective effort and flow variables associated with the respective bonds. In Figure 5.4, these bonds are numbered 1, 2, 4 and 5. In Figure 5.8, these bonds are numbered $3,4,5,7,10,11,12,14,17,18,19,21,24,25,26,28,31,32,33,35,38$, $39,40,42,45,46,47$ and 49.

Using the techniques developed by Karnopp, et al (1990), the 4 state equations for Figure 5.4 and 28 state equations for Figure 5.8 are derived directly from the bond graphs. After formulation, the 4 and 28 state equations were reduced to 3 and 21 , respectively. This was justified since the equations were equal. These state equations, using the notation 
in the Figures, are listed in general form in Appendix C. These equations are then written in general state-space form with " $\mathrm{r}$ " sensors and " $\mathrm{m}$ " actuators as

$$
\begin{aligned}
& \dot{\tilde{x}}=\tilde{A} \tilde{x}+\tilde{B} \tilde{u} \\
& \tilde{y}=\tilde{C} \tilde{x}
\end{aligned}
$$

where,

$\dot{\tilde{x}}=$ time derivative of the state vector (n),

$\tilde{x}=$ state vector $(\mathrm{n})$,

$\tilde{A}=$ system matrix (nxn),

$\tilde{B}=$ input matrix $(\mathrm{nxm})$,

$\tilde{C}=$ output matrix (rxn),

$\tilde{u}=$ input vector $(\mathrm{m})$, and

$\tilde{y}=$ output vector $(r)$.

Using the technique described above, two models have been developed which can be used to study the dynamics of deformable mirrors. The first model, shown in Figure 5.3 , models a single actuator and plate stiffness. The actuator is modeled as a combination of a C-field, mass and dashpot. The C-field models the electro-mechanical effect of the actuator and utilizes an energy function which satisfies Maxwell's reciprocity (Karnopp, 1996). The development of this relationship is described in Appendix B. The actuator mass is modeled as an effective actuator mass due to the fact that the entire actuator mass is not moving at the same velocity. A similar approach to modeling the mass was completed by Marlow (1994). The dashpot allows different types of resistances to be modeled. For example, linear damping could be used or a more complex non-linear friction law which would produce hysteresis is possible. Lastly; the plate compliance and mass is simply modeled as a compliance and a mass. The mass of the actuator and plate is lumped into a single effective mass. 
The model represented in Figure 5.3 is also shown in Bond Graph form in Figure 5.4. As seen in this figure, the C-field connects bond 4 (actuator force and velocity) and bond 5 (actuator voltage and current). Bond 6 connects to the electrical resistance in the driving circuit. The inertia element connected to bond 2 and the resistance element connected to bond 3 are part of the actuator model and bond 2 represents a combined inertia term for both the actuator and plate. The plate's compliance is shown connected to bond 1 . The dynamic equations of motion were developed utilizing the bond graph of Figure 5.4 and can seen in Appendix C.

These linear equations were coded using Matlab to simulate the frequency response shown in Figure 5.5 and the step response shown in Figure 5.6. The parameters used in the model were based on information on the National Ignition Facility prototype thirty-nine actuator deformable mirror and these are listed in Appendix D.

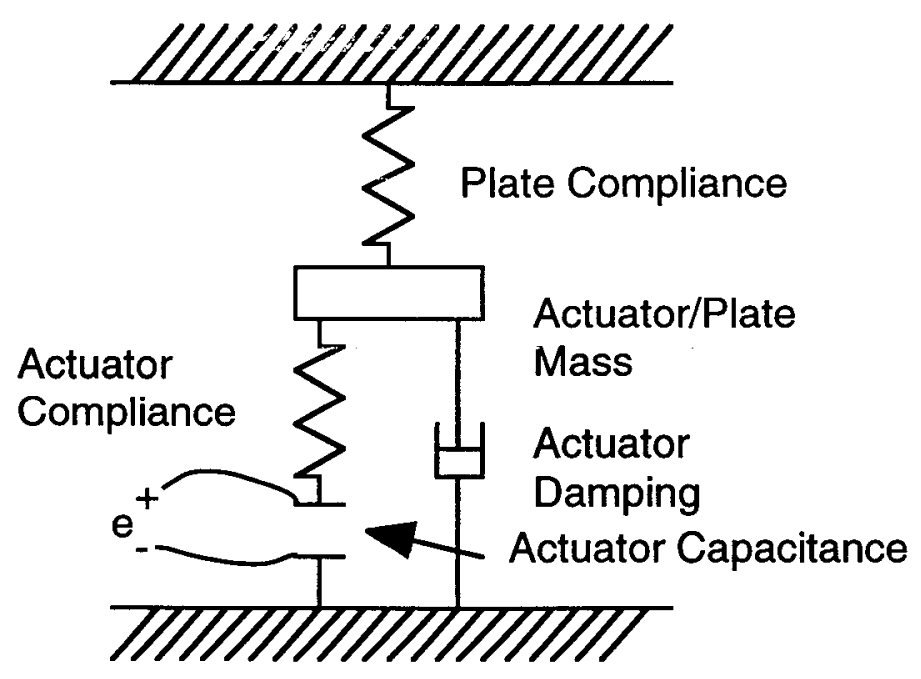

Figure 5.3 Simplified actuator and plate model. 


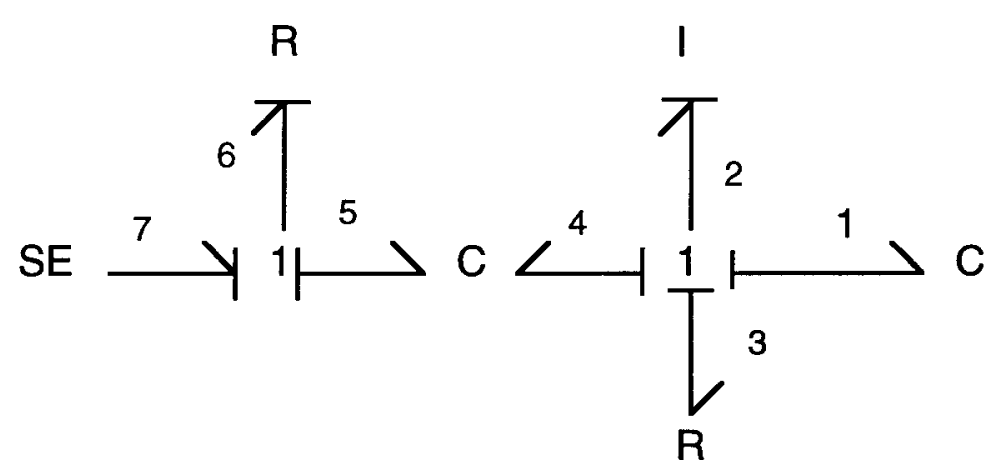

Figure 5.4 Bond graph of Figure 5.3
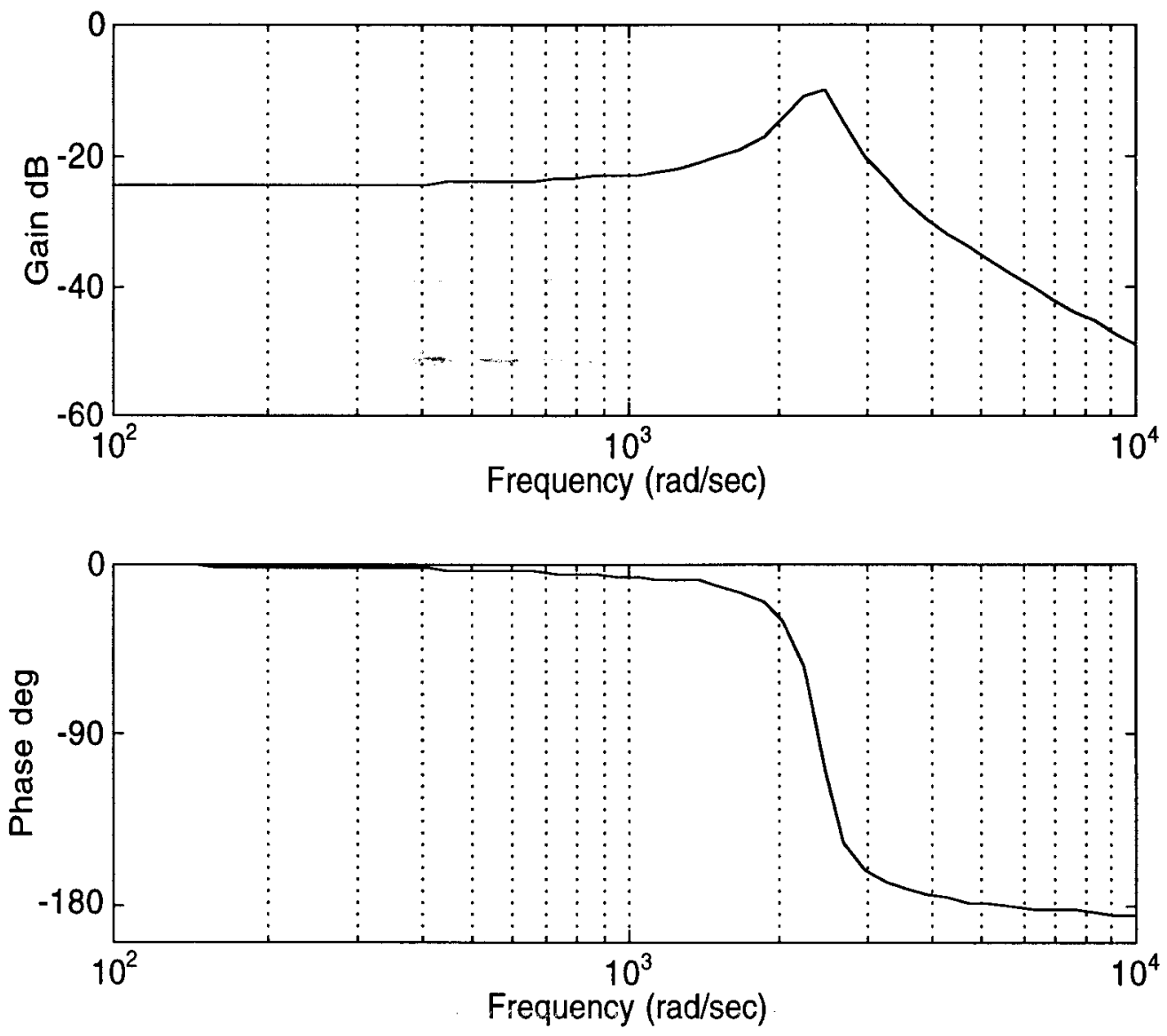

Figure 5.5 Bode plot of single actuator system. 
The second model, shown in Figure 5.7, uses seven actuators and a linear elastic plate. Note that the seven actuator pattern is a segment of the NIF thirty nine actuator deformable mirror, except that the edge boundary conditions are free. The actuators are identical to the one described earlier and a $\mathrm{C}$-field is used to model the plate. The C-field is developed through the use of superposition; therefore, we assume operation in the linear range of strains and deflections.

The finite element and experimental results of Chapter 3 were utilized in developing the C-field. In this model, the C-field matrix is represented in the "stiffness form", which means the constitutive law gives force as a function of displacement. The interested reader is referred to Karnopp, et al (1990) for details on developing this multi-port C-field. The detailed inertia effects of the plate have been neglected, assuming that the mode shape frequencies were significantly higher than the frequencies of interest. This assumption relates to the model reduction approach, which assumes we are in region II of Figure 5.2.

A bond graph representation of this model is shown in Figure 5.8. As evident by comparing Figures 5.4 and 5.8, the actuator components are the same for the single actuator model and the seven actuator model. It should be mentioned that the seven actuator model could easily be expanded to any number of actuators. With the state equations developed from Figure 5.8 and then programmed in Matlab (see Appendix D), open loop simulations were completed. Development of the state equations can be found in Appendix C. Figures 5.9 and 5.10 show the singular value plot, which represents the family of frequency plots for a MIMO system, and step response, respectively, for the seven actuator plate system. The general characteristics of these plots change as system parameters are adjusted; e.g., reducing the ratio of the plate stiffness to the actuator stiffness causes the actuator coupling to be reduced. Although not shown here, the result of this can be seen in the singular value plot as the singular values spread further apart. The actuator coupling relates to the influence functions, which is the defining characteristic of a deformable mirror. 


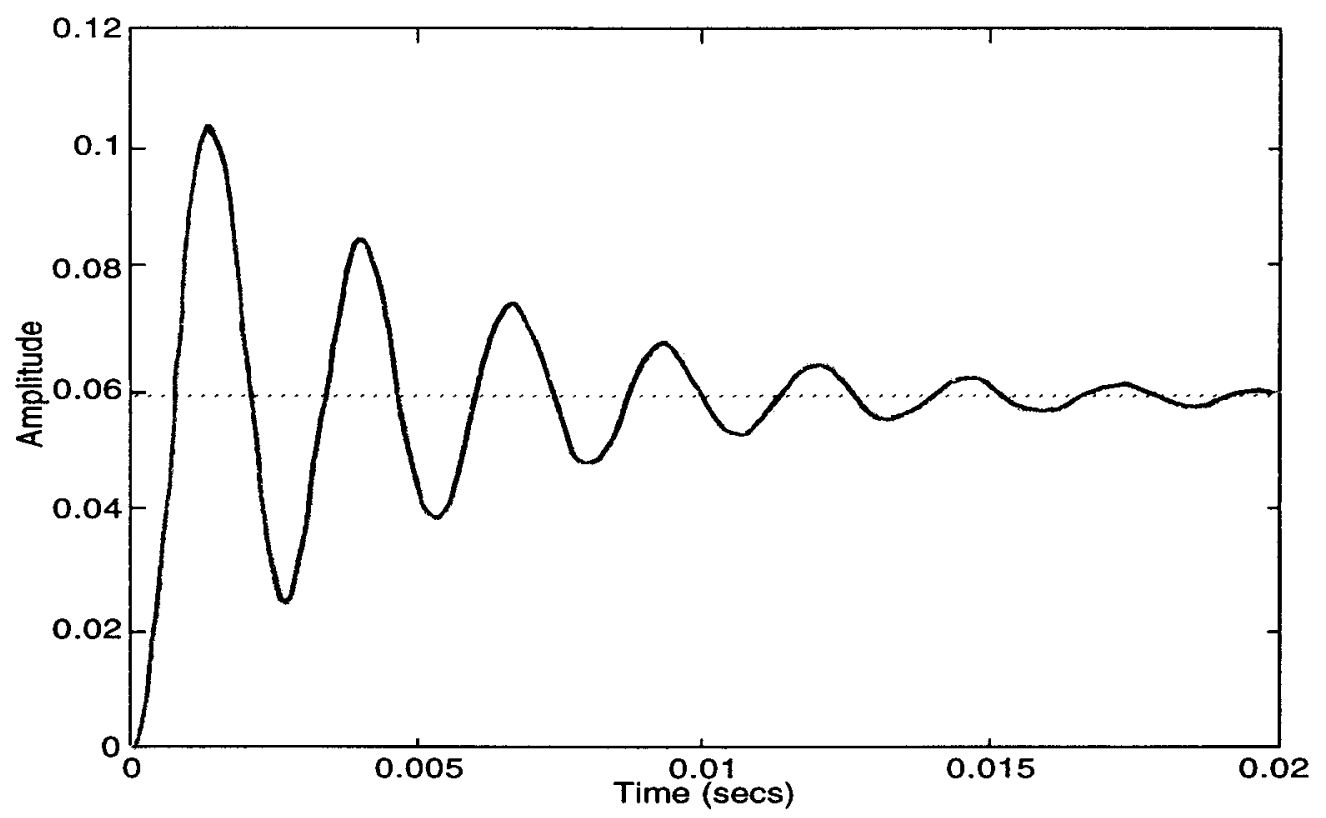

Figure 5.6 Step response for single actuator system

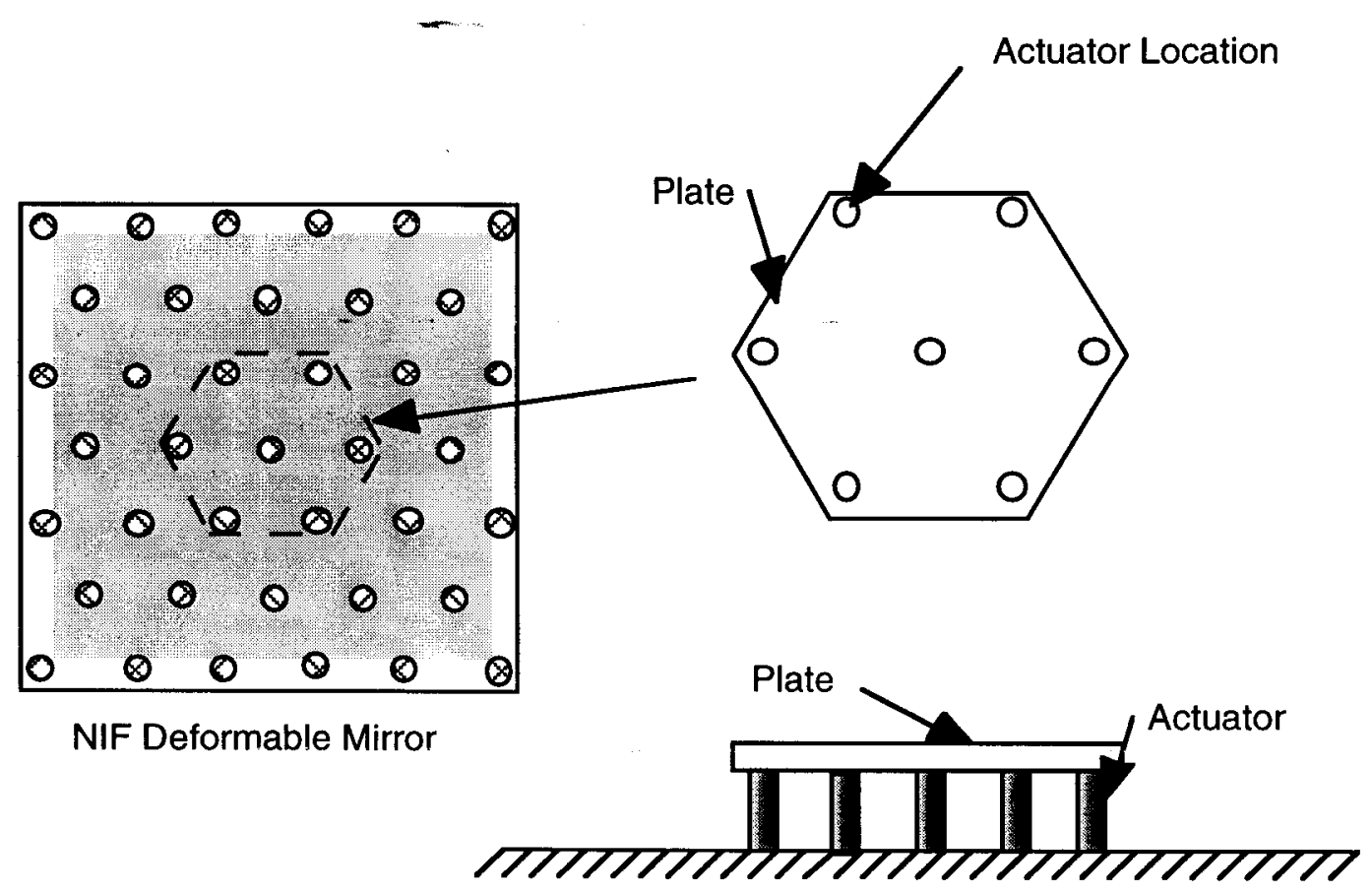

Figure 5.7 Actuator and plate model 


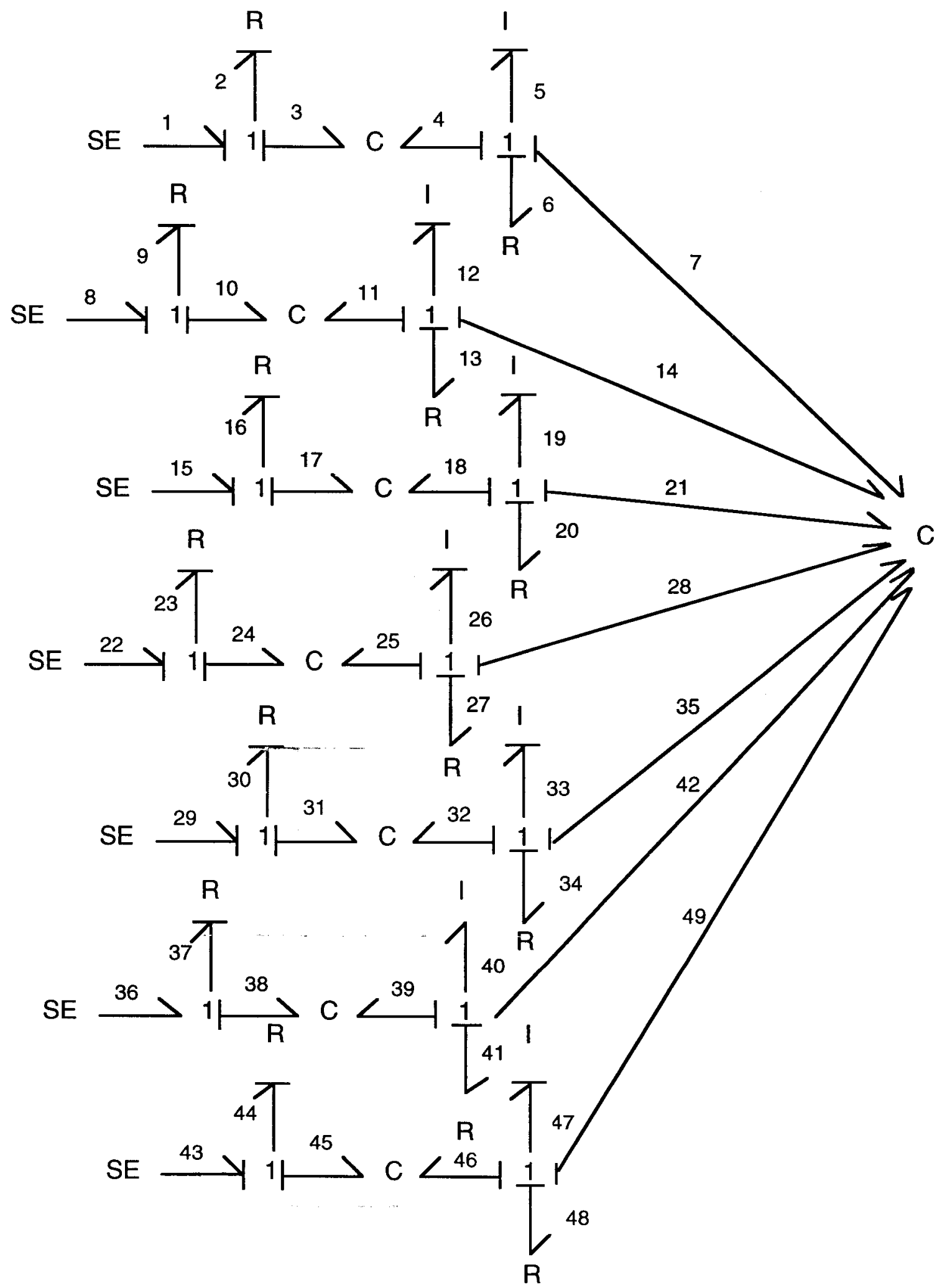

Figure 5.8 Bond graph of Figure 5.7 


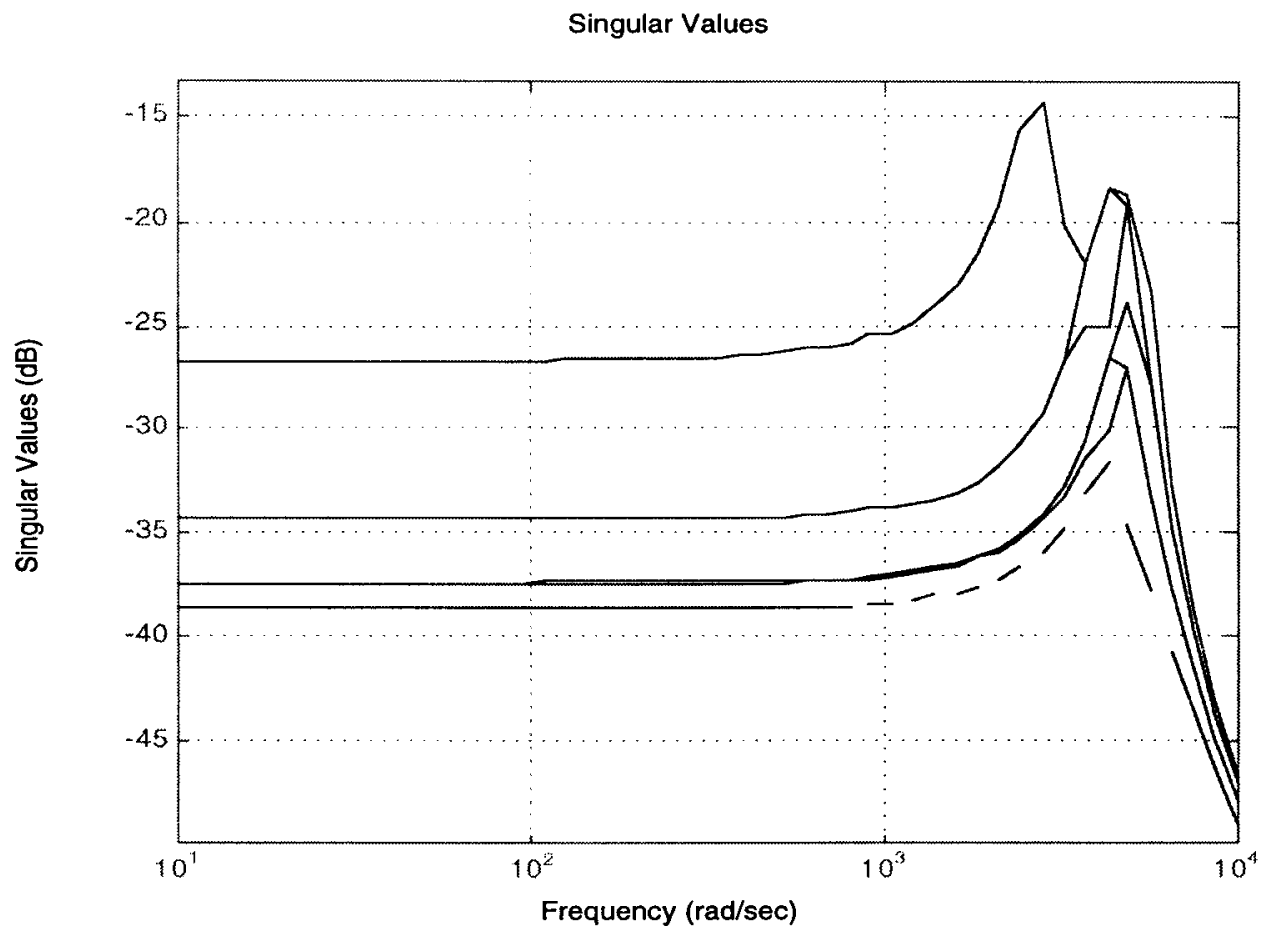

Figure 5.9 Singular value plot for seven actuator plate model

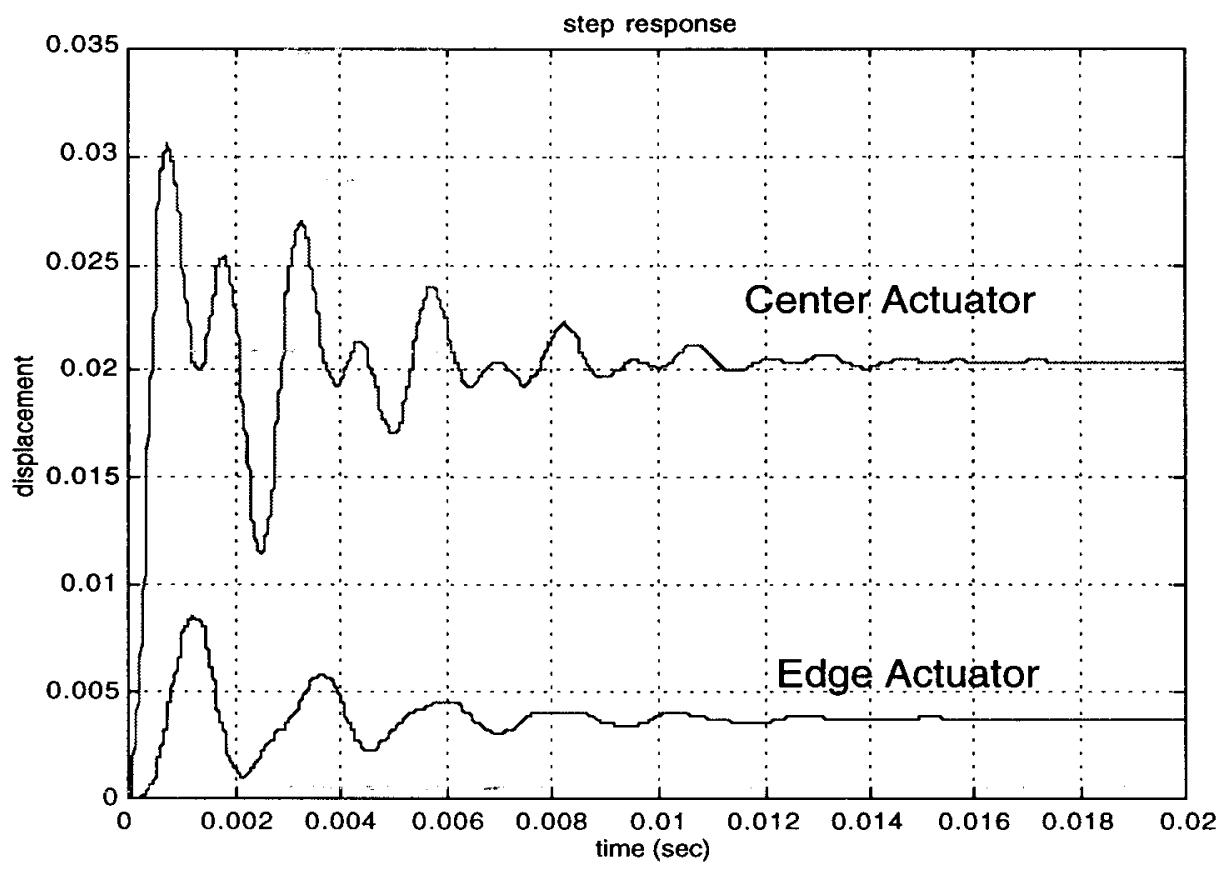

Figure 5.10 Step response for seven actuator plate model, with input to center actuator 


\subsection{Experimental Verification}

The purpose of this section is to utilize experimental data collected on the NIF thirty nine actuator prototype deformable mirror to validate the single actuator and seven actuator plate models. The dynamic behavior of the influence function, which is the deformed shape when one actuator is displaced and all others are held constant, will be studied. Experimentally, the dynamic behavior of the influence function has been studied by Freeman and Garcia (1982) and Marlow (1994). Experiments for this thesis focused on acquiring the frequency response of various actuators, such as actuator number twenty as shown in Figure 3.3. This work was completed at Raytheon Optical Systems, Incorporated and was part of their acceptance test procedures for building a prototype large aperture deformable mirror for the National Ignition Facility (La Fiandra, et al, 1998). This data was corrupted due to poor mounting procedures and is therefore only useful as a qualitative measure. Future tests should be completed to generate data for a quantitative comparison.

Figure 5.11 depicts a block diagram of the experimental setup. As seen in Figure 5.12 and 5.13, a Fotonics displacement sensor and data acquisition system were configured to acquire frequency response data. A low amplitude sinusoidal excitation frequency was applied to the PMN actuators, and the Fotonics sensor recorded the displacement as the frequency was swept from 1 hertz to 1 kilo-hertz and from 1 kilo-hertz to 10 kilo-hertz. The frequency sweep of the Dynamic Signal Analyzer was set up to drive the high voltage amplifier, and the PC was commanded to bias its output to approximately 54 volts. This output was applied to the actuators via the actuator selection box. The feedback output of the high voltage amplifier was connected to channel 1 of the analyzer and became the reference signal. The Fotonic sensor output was connected to channel 2 of the Analyzer as the test signal. After the sensor was positioned at the actuator to be tested, it was calibrated before any frequency sweep was begun. A representative plot can be seen in Figure 5.14. 


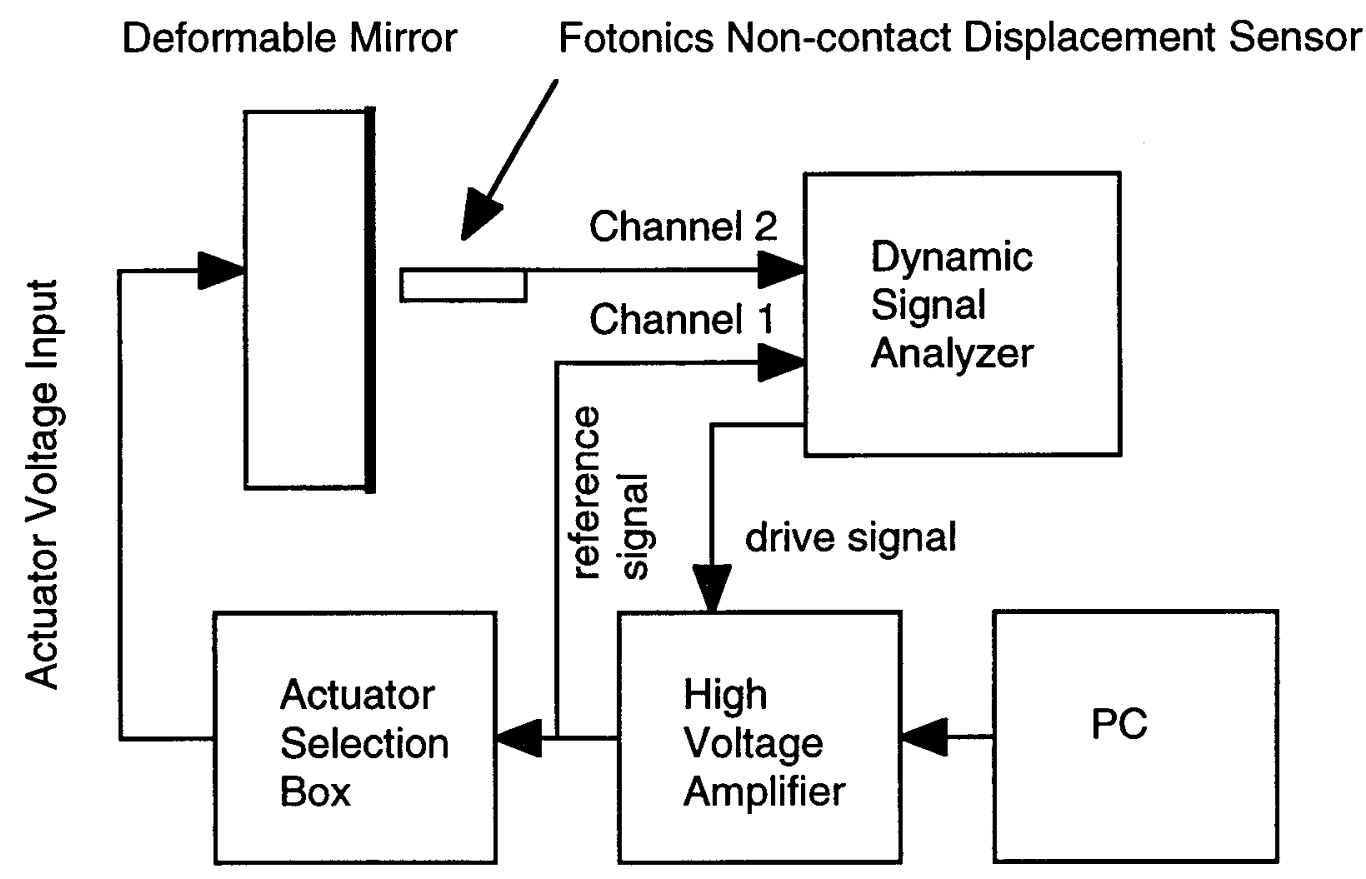

Figure 5.11 Block diagram of experimental setup

These experimental results were qualitatively compared to the models developed previously in this chapter and show very good agreement. When comparing Figures 5.9 and 5.14, the seven actuator plate model response and the experimental response, respectively, the following observations are made. The general shape of the responses are similar in that they are constant until approximately 2 kilo-hertz and then roll off at approximately $-40 \mathrm{~dB}$ per decade. Furthermore, these results compare qualitatively to Marlow's (1994) experimental and modeling frequency response results. Although Marlow's (1994) model and parameter values are different, the general frequency response shape, reasonance peak, and roll off characteristics show reasonable agreement with the models developed in this chapter. Figure 5.15 depicts a frequency response plot from Marlow (1994). 
On the basis of the experimental results, model parameters were adjusted using a trial and error approach to match the system experimentally measured response. These model parameters can be found in Appendix $\mathrm{D}$ and $\mathrm{E}$. The damping value was the primary adjustment parameter and was also the largest modeling uncertainty. The effective stiffness and mass parameters were also slightly adjusted to match the resonant frequency. The same approach was utilized for the single actuator model. This experimental validation gives confidence for future use of these models for control development purposes.

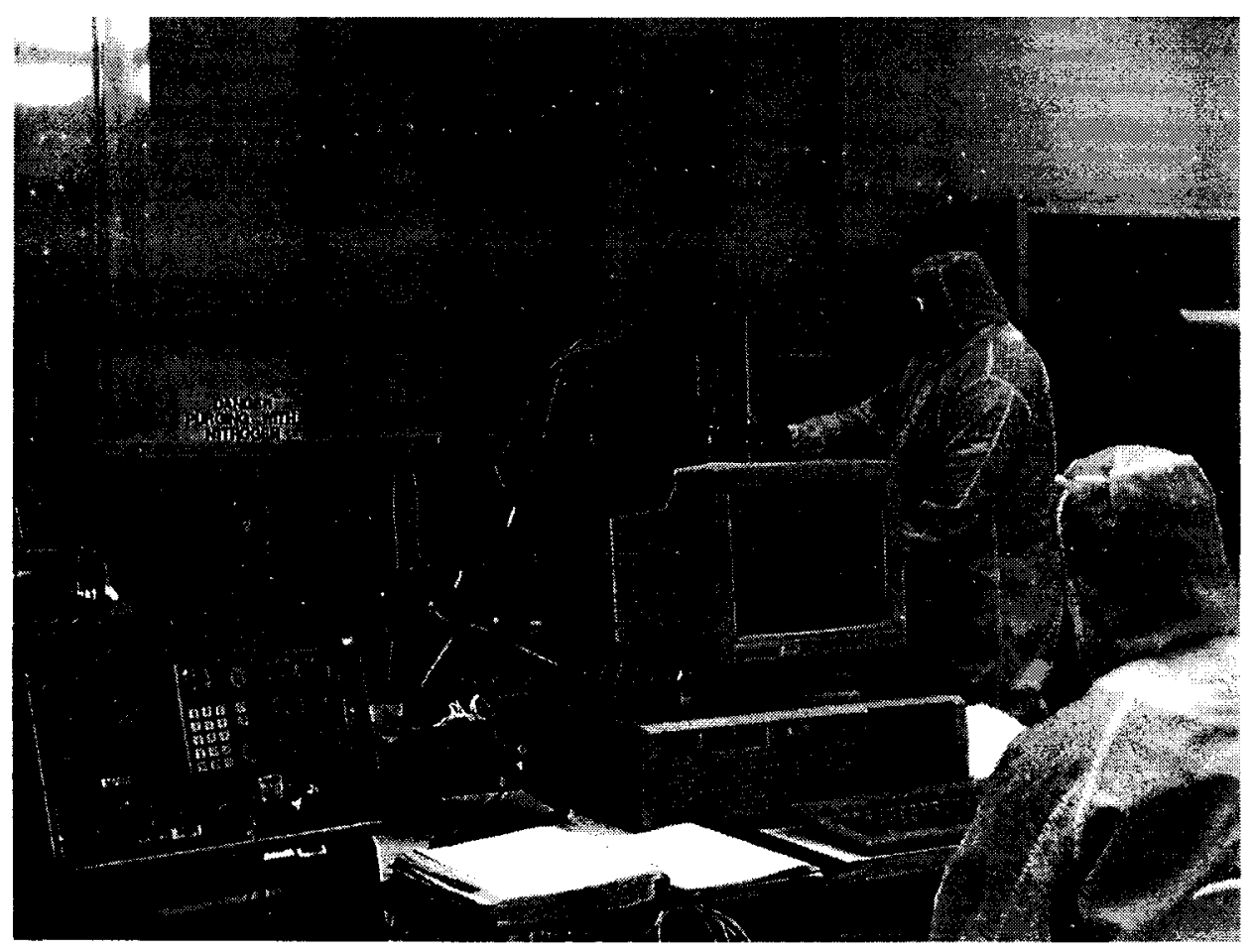

Figure 5.12 Experimental setup for frequency response test 


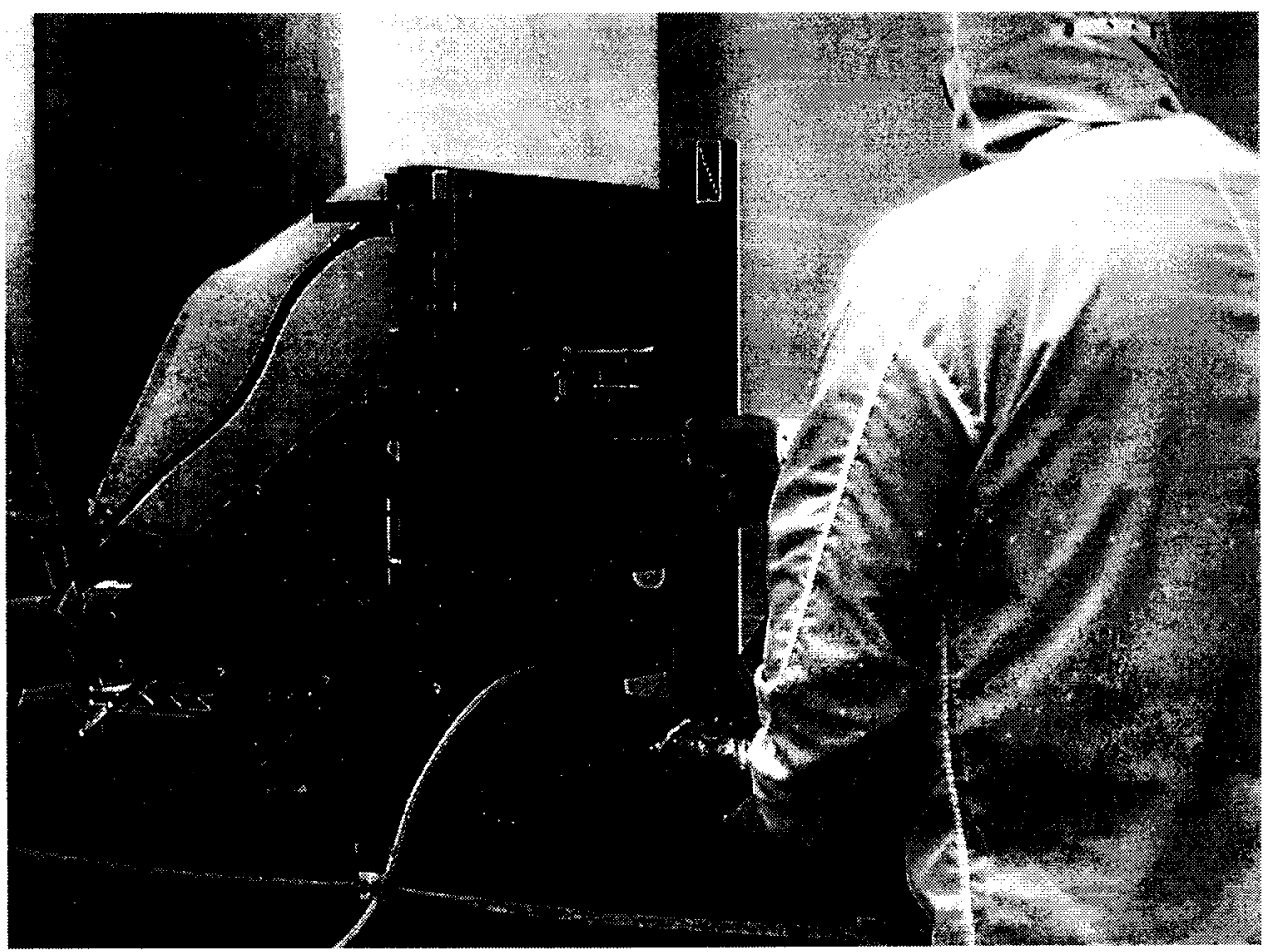

Figure 5.13 Detail of experimental setup.

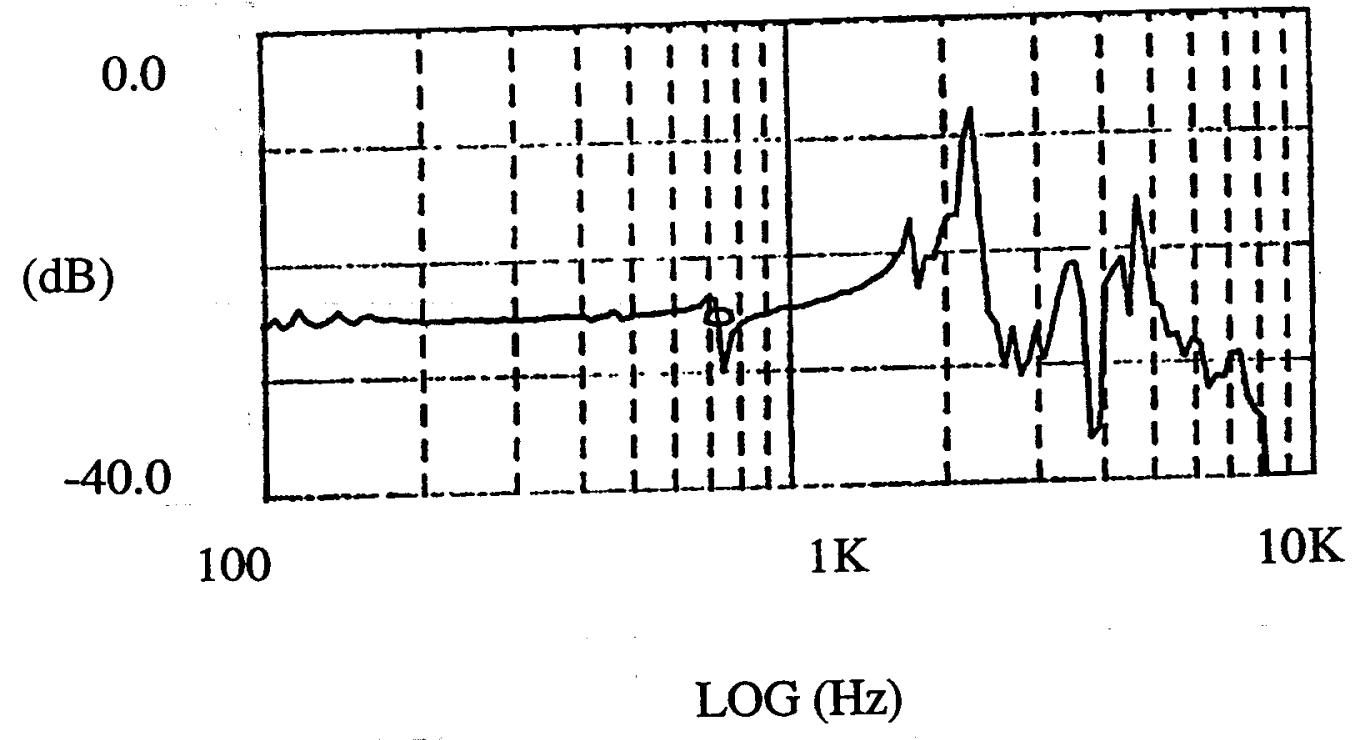

Figure 5.14 Experimental frequency response data 


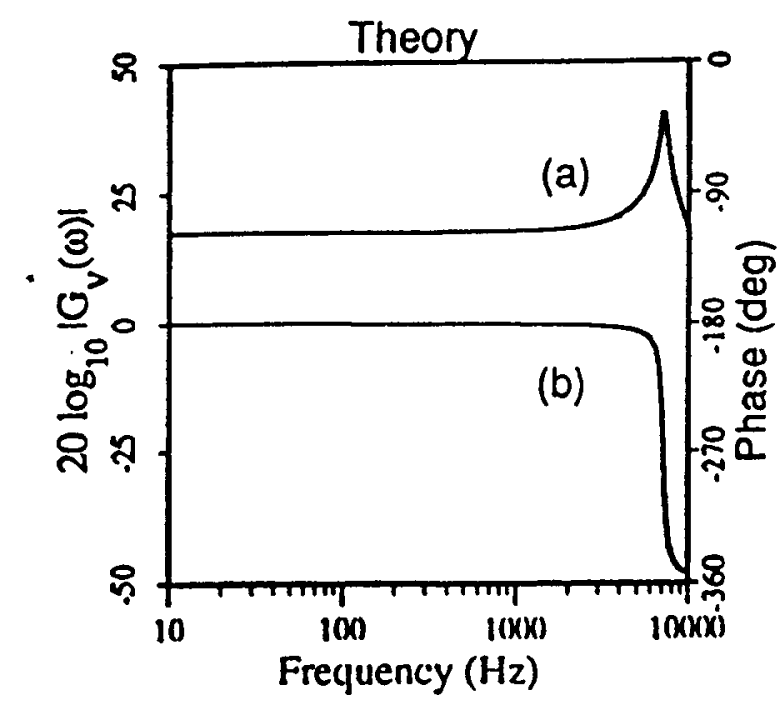

Figure 5.15 Frequency response plot from Marlow (1994)

\subsection{Conclusion}

In this chapter two computer models were developed which will facilitate the development of dynamic surface shape controllers. The first part of this chapter covered the general model development approach and overviewed previous approaches. Next, model reduction techniques were reviewed and a modal density selection approach was employed. The third section covered the derivation of dynamical equations of motion utilizing bond graph techniques for a single actuator and multiple actuator system. These equations were coded in Matlab, and frequency and step responses were completed. Lastly, the dynamic equations of motion was qualitatively verified by showing reasonable agreement with experimental and published literature results. 


\section{CHAPTER 6 - DYNAMIC SURFACE SHAPE CONTROL}

\subsection{Introduction}

The purpose of this chapter is to develop a multi-input multi-output (MIMO) controller for use in surface shape control which will improve closed loop positioning accuracy in the presence of disturbances and uncertainties. Initially, general design objectives are overviewed with emphasis placed on stability, performance and robustness. Next, a SISO controller is developed based on a proportional-integral approach, which will give general insight to the characteristics of the system. Then, MIMO controllers are developed based on proportional-integral (PI) and $\mathrm{H}_{\infty}$ methods utilizing the dynamic model from Chapter 5. Lastly, performance comparisons are made between MIMO controller designs and robustness evaluated.

\subsection{Control Approach and Related Research}

Surface shape control may involve tens to hundreds of degrees of freedom systems and require high precision and accuracy. This poses a difficulty for control system engineers in that developing accurate high order models and then MTMO controllers can be almost impossible. Most development to date by-passes any significant modeling and analysis prior to fine tuning controllers once the system has been built. This work will focus on developing a MIMO modern controller to improve closed loop performance and robustness. Performance and robustness comparisons are made between MIMO designs using proportional-integral (PI) and $\mathrm{H}_{\infty}$ methods.

Research related to the development of control approaches vary significantly. There are a significant number of control approaches for SISO systems, examples include but are not limited to, P.I.D, optimal control, sliding mode control and adaptive control (Astrom, 1983; Banyasz and Keviczky, 1993; Ellerbroek, 1994; Luo and Delasen, 1993; Peter and Iserman, 1993; Rad and Low, 1995; Ratten, 1989). Balas and Doyle (1990), Bushnell 
(1979), Hyde and Seering (1991), Fanson, et al (1990), Lyakhov, et al (1994), Schaechter (1981), and Shishakov and Shmal'gauzen (1992) have investigated various control problems related to flexible structures or plates. Fewer approaches have been developed and utilized for MIMO systems. Examples include, but are not limited to, $\mathrm{H}_{\infty}$ synthesis, $\mathrm{Q}-$ factorization and Youla synthesis (Mayne, 1996; Safonov, et al, 1989; Doyle, et al, 1989; Sueur and Dauphintanguy, 1991; Brewer, 1995). Although the control approaches vary significantly, in general, they attempt to accomplish the same goals (e.g., stability, robustness, disturbance rejection, tracking).

\subsection{Control Model}

As mentioned, the main focus in this final phase of the research is to develop a controller which has robust characteristics for dynamic surface shape control. The general block diagram describing this control system is shown in Figure 6.1, where,

$$
\begin{aligned}
& \tilde{r}=\text { reference input vector }(\mathrm{r}), \\
& \tilde{e}=\operatorname{tracking} \text { error vector }(\mathrm{r}), \\
& \tilde{G}_{c}=\text { controller matrix }(\mathrm{mxr}), \\
& \tilde{u}=\text { input vector }(\mathrm{m}), \\
& d \tilde{u}=\text { input disturbance vector }(\mathrm{m}), \\
& \tilde{G}_{p}=\text { plant matrix }(\mathrm{rxm}), \\
& d \tilde{y}=\text { output disturbance }(\mathrm{r}), \\
& \tilde{y}=\text { plant output vector }(\mathrm{r}), \\
& \tilde{n}=\text { sensor noise vector }(\mathrm{r}), \text { and } \\
& \tilde{G}_{s}=\text { sensor matrix }(\mathrm{rxr}) .
\end{aligned}
$$


The main focus of this chapter is on the development of $\tilde{G}_{c}$, the controller matrix, using a traditional proportional-integral (PI) technique and the more modern approach of $\mathrm{H}_{\infty}$. The plant matrix, $\tilde{G}_{p}$, will use the state space model developed in Chapter 5. Lastly, the sensor matrix $\tilde{G}_{s}$ is assumed to be unity (identity matrix for MIMO case) for this analysis and represents a strain gauge, although it could easily be modified to incorporate sensor dynamics or a Shack-Hartmann sensor as implemented in the static control method of Chapter 4.

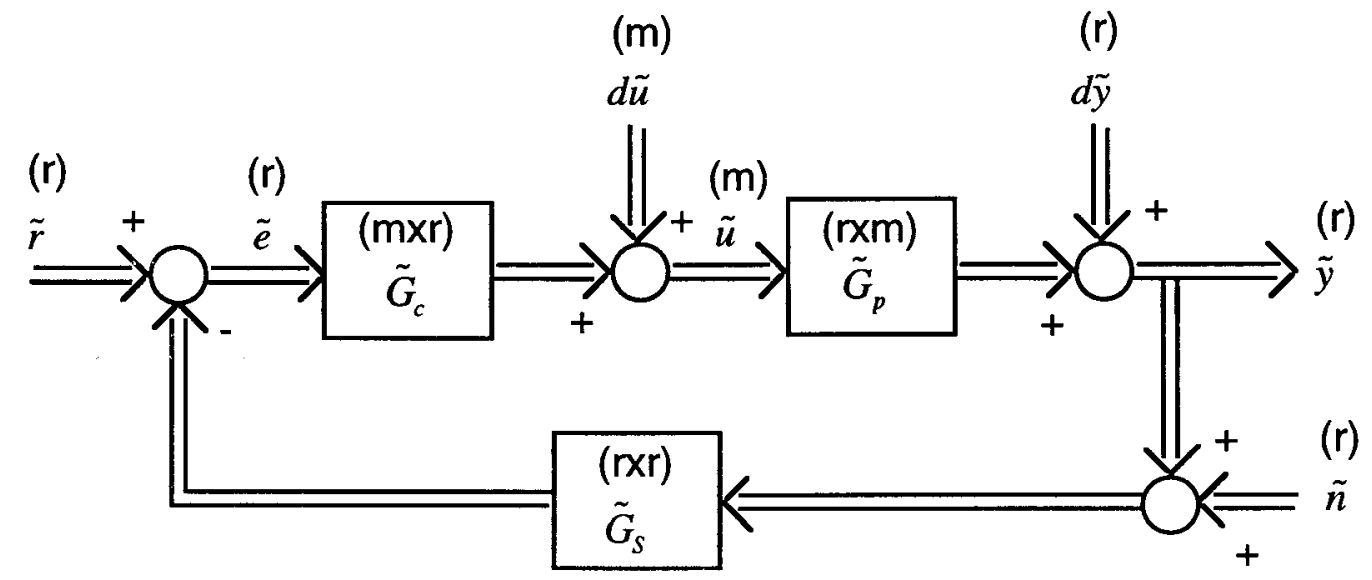

Figure 6.1 General MIMO feedback loop

\subsection{Design Objectives}

The purpose of this section is to overview the general controller design objectives. However, before presenting these objectives, some general transfer function relationships are presented. Using Figure 6.1, transfer functions can be derived (Brewer, 1995) and are defined as 


$$
\begin{aligned}
& L_{y} \equiv \tilde{G}_{p} \tilde{G}_{c}, \\
& T_{y} \equiv\left(I+L_{y}\right)^{-1} L_{y}, \\
& S_{y} \equiv\left(I+L_{y}\right)^{-1}, \\
& L_{u} \equiv \tilde{G}_{c} \tilde{G}_{p}, \\
& T_{u} \equiv\left(I+L_{u}\right)^{-1} L_{u}, \text { and } \\
& S_{u} \equiv\left(I+L_{u}\right)^{-1}
\end{aligned}
$$

where $L_{y}$ and $L_{u}$ are defined as the return ratio at the plant output node and plant input node, respectively. The $T$ 's and $S$ 's are defined as the complimentary sensitivity function and sensitivity function, respectively, with the subscripts defining the node location. Equations 6.1 through 6.6 are used to define general performance design objectives.

The general design objectives are split into four areas: 1) Stability, 2) Performance, 3) Conflicts, and 4) Robustness (Mayne, 1996). Next, the four areas are reviewed:

1) Stability is the first and overriding objective. In general there are two distinct concepts of stability. The first is intrinsic stability, based on the systems differential equations, which examines the poles of the system matrix. The second is referred to as input-output stability and is based on input-output behavior. The second approach defines stability in terms of the poles of transfer function matrices (Mayne, 1996).

2) Performance is split into four additional areas: a) Tracking, b) 'Good' noise and disturbance rejection, c) Disturbances/noise effects on the error signals, and d) Disturbances/noise effects on actuators. Tracking is defined by Eqn. 6.2 and is the transfer function $\tilde{r} \rightarrow \tilde{y}$. Good tracking requires Eqn. 6.2 to be approximately unity in the frequency domain of interest, which is generally at low frequencies. Noise rejection is defined by Eqn. 6.2 and is the transfer function $\tilde{n} \rightarrow \tilde{y}$. Good noise rejection requires Eqn. 6.2 to be small in the frequency of interest, which is generally at high frequencies. 
Disturbance rejection is defined by Eqn. 6.3 and is the transfer function $d \tilde{y} \rightarrow \tilde{y}$. Good disturbance rejection requires Eqn. 6.3 to be small in the frequency range of interest, which is generally low frequencies. Disturbances/noise effects on the error signals are not usually of direct interest. However, it is a useful intermediary for assessing the effects of noise and disturbances on the plant input. Eqn. 6.3 is the transfer function between $\tilde{r} \rightarrow \tilde{e}$, and is a command following measure. Disturbances/noise effects on the actuators are of significant interest. Eqn. 6.6 is the transfer function between $d \tilde{u} \rightarrow \tilde{u}$ and should be small in the frequency range of interest. The transfer function between $\tilde{r} \rightarrow \tilde{u}$ is defined as

$$
\tilde{u}=S_{u} \tilde{G} \tilde{r}
$$

and should be small enough not to saturate the actuators. Lastly, the transfer function between $\tilde{n} \rightarrow \tilde{u}$ is defined as

$$
\tilde{u}=-S_{u} \tilde{G}_{c} \tilde{n}
$$

and should be small over the frequency range of interest.

3) Conflicts exist for both stability and performance objectives. Many of the performance objectives conflict with each other and these conflicts are expressed by

$$
S_{y}+T_{y}=I
$$

and/or

$$
S_{u}+T_{u}=I
$$

Several of the objectives require the sensitivity function, $S$, to be small and the complimentary sensitivity function, $T$, to be unity, and in view of Eqn.'s 6.9 and 6.10, are 
consistent. However, some objectives require both $T$ and $S$ to be small, which is impossible to have at a given frequency. Fortunately, many objectives are achieved by meeting the condition over specified frequencies. For example, tracking usually requires $S$ to be small at low frequencies, and sensor noise rejection usually requires $T$ small at high frequencies. Lastly, stability also conflicts with performance objectives. For example, a high gain controller often causes instability.

4) Robustness is defined as the ability to maintain the properties of stability and performance as the plant ranges over a set of plants, relative to the nominal plant. The model of the plant, on which the design is based and inevitably has errors, does not behave identically to the "real" plant being controlled. It is essential that the design objectives, stability and performance, are robust. Therefore, it can be stated that a controller provides robust stability and performance, if it stabilizes and ensures a given minimum performance for every plant within the set (Mayne, 1996).

\subsection{SISO Control Simulation}

Due to the complexity of developing controllers for large complex systems, a simple representative single-input single-output (SISO) system will be studied to determine general characteristics of the system. The system dynamic equations were developed in Chapter 5 and the state space equations can be found in Appendix D. The primary value in studying this simplified SISO system is to examine the ability to develop a SISO controller and then infer the controller characteristics in a multi-input multi-output (MIMO) design.

As with all control development, both time domain and frequency domain responses will be used to evaluate the performance of the developed controller, as the time domain response gives good transient measures and the frequency response gives good performance measures. A common way to specify desired closed-loop performance is by a step response test. For this single actuator system, the specification is that a step reference input $\tilde{r}$ should produce a plant output $\tilde{y}$ satisfying 
settling time $=\tau$ seconds

and

$$
\text { overshoot }<=\lambda \text { percent. }
$$

This will be accomplished by shaping the complimentary sensitivity $T(s)$, the transfer function from $\tilde{r}$ to $\tilde{y}$, so that it approximates a standard second order system. Accordingly, the ideal $\mathrm{T}(\mathrm{s})$ is represented as

$$
\mathrm{T}(\mathrm{s})=\frac{w_{n}^{2}}{s^{2}+2 \zeta w_{n} s+w_{n}^{2}}
$$

A settling time of $\tau$ seconds requires

$$
\frac{4}{\zeta w_{n}} \approx \tau
$$

and an overshoot of $\lambda$ requires

$$
\exp \left(\frac{-\zeta \pi}{\sqrt{1-\zeta^{2}}}\right)=\lambda
$$




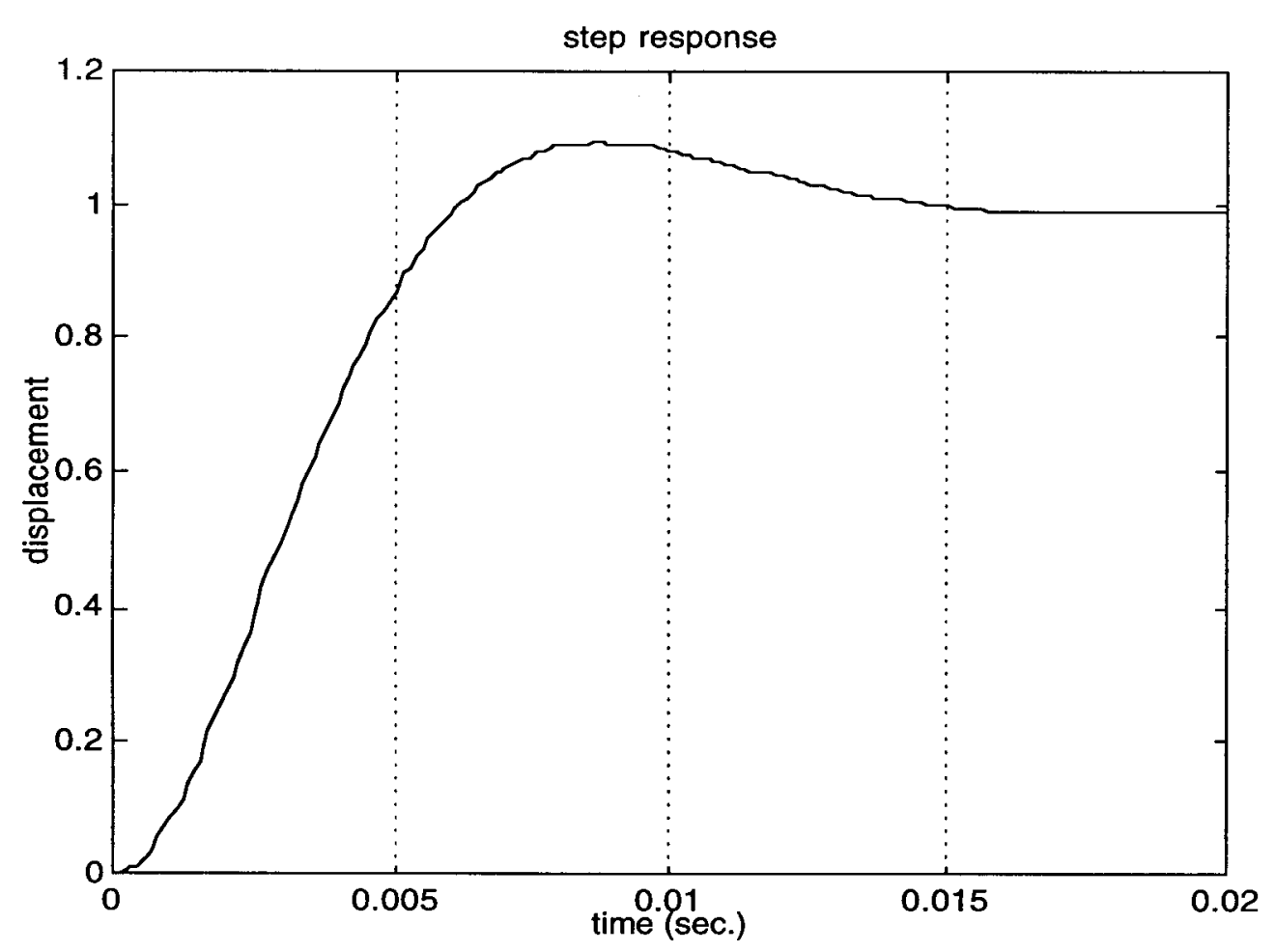

Figure 6.2 Step response $(y / r)$ of ideal system to be matched

Next, the settling time and overshoot was set to $\tau=0.015$ seconds and $\lambda=10 \%$, respectively, and substituted into Equations 6.12 and 6.13. These values were selected based on the open loop response characteristics, such as the rise time and bandwidth, and the desire to keep the overshoot reasonably small. Solving Equations 6.12 and 6.13 gives $\zeta=0.5912$ and $w_{n}=451.05$. These numbers will be rounded to $\zeta=0.6$ and $w_{n}=450$. Therefore, the ideal $\mathrm{T}(\mathrm{s})$ is

$$
\mathrm{T}(\mathrm{s})=\frac{202500}{s^{2}+540 s+202500}
$$

and is shown in the time domain in Figure 6.2 and in the frequency domain in Figure 6.5. Furthermore, the ideal sensitivity function is written as 


$$
\mathrm{S}(\mathrm{s})=1-\mathrm{T}(\mathrm{s})=\frac{s^{2}+540 s}{s^{2}+540 s+202500}
$$

and is shown in the time domain in Figure 6.3 and in the frequency domain in Figure 6.6. Now, using the ideal second order system responses, a matching technique (Brewer, 1991) will be used to determine the gains in a proportional-integral (PI) controller. Often, the open loop transfer function, $\mathrm{L}(\mathrm{s})$, is used to help facilitate the matching process. This transfer function is shown in Figure 6.4.

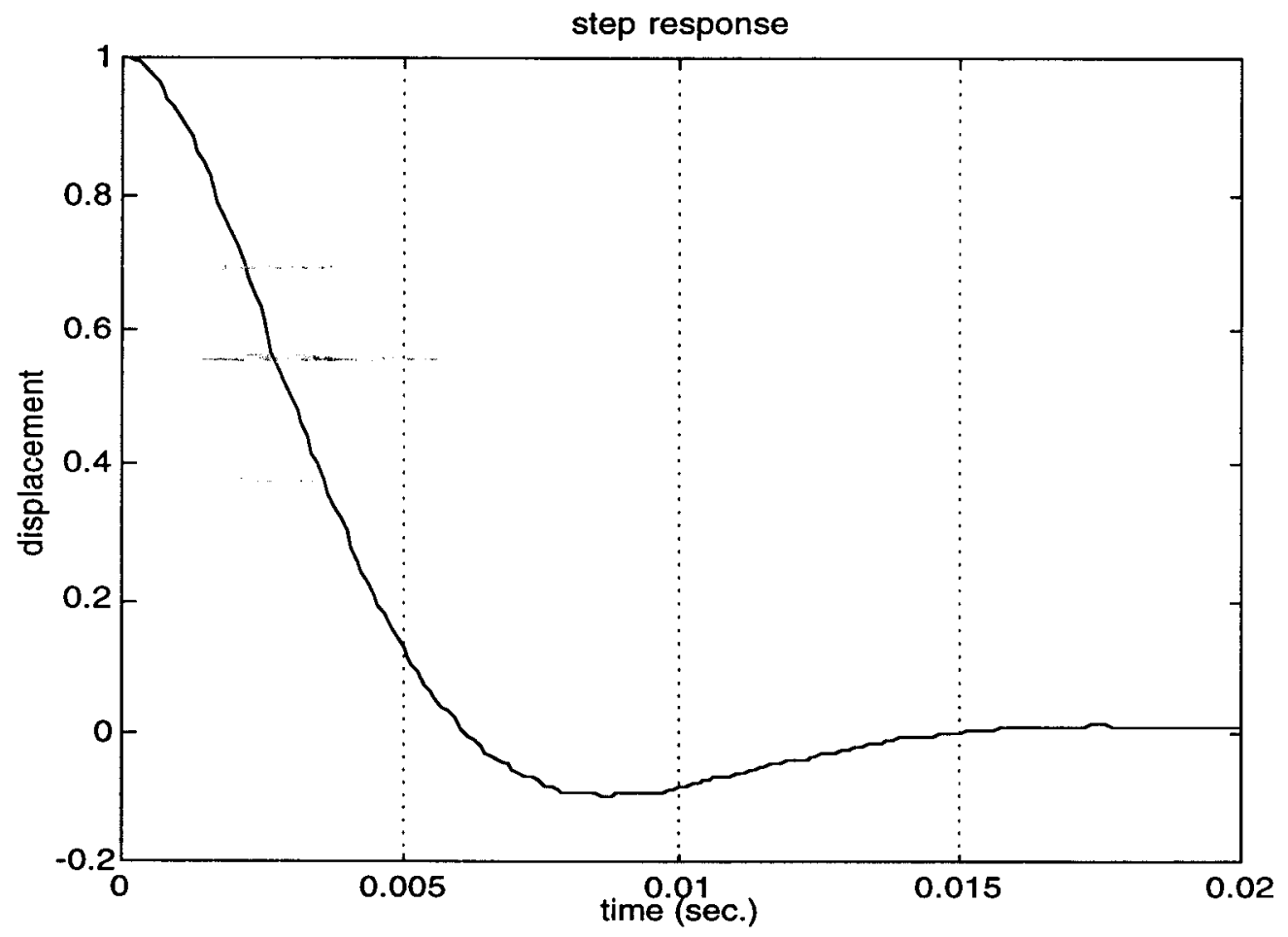

Figure 6.3 Step response $(y / d)$ of system to be matched

Using a standard proportional plus integral (PI) controller model, the controller gains $K_{p}$ and $K_{i}$ were adjusted to achieve a response similar to the matching system 
response. After many iterations, the gains were selected as $K_{p}=0.01$ and $K_{i}=200$, which gave a response which followed the matching system reasonably close. The step response of the closed loop, open loop and matching system is shown in Figure 6.7. The closed loop system reduced the overshoot from approximately $75 \%$ to zero and the settling time was maintained per the specification. Due to the light damping in the open loop system, it was difficult to significantly improve the settling time beyond the specification, without introducing significant oscillations in the response. It should also be noted that the rise time was increased over the open loop system, which is expected and is the trade-off for reducing the overshoot.

Comparing the responses in the frequency domain shows similarities, as would be expected from the time domain responses. Comparing Figures 6.5, the matching system complimentary sensitivity plot, and Figure 6.8, the designed complimentary sensitivity plot, shows a difference in tracking response which corresponds to the rise time difference. Also, notice the sharp peak in Figure 6.8 at approximately 2 kilo-hertz. This represents the difficulty in trying to match perfectly to a second order system.

Comparing Figure 6.6, the sensitivity transfer function for the matching system, and Figure 6.9, the sensitivity transfer function for the designed system, shows both systems have reasonably good disturbance rejection at low frequencies, and they slightly amplify the disturbance in a small frequency band.

Lastly, Figures 6.4 and 6.10, show the system open loop transfer function "L" for the matching system and designed system, respectively. In general, the shape looks good, as the gain is "high" at low frequencies and "low" at high frequencies. The designed system, Figure 6.10, also shows small attenuation around the 2 kilo-hertz range, which would indicate the need for a higher order controller to achieve better attenuation. 

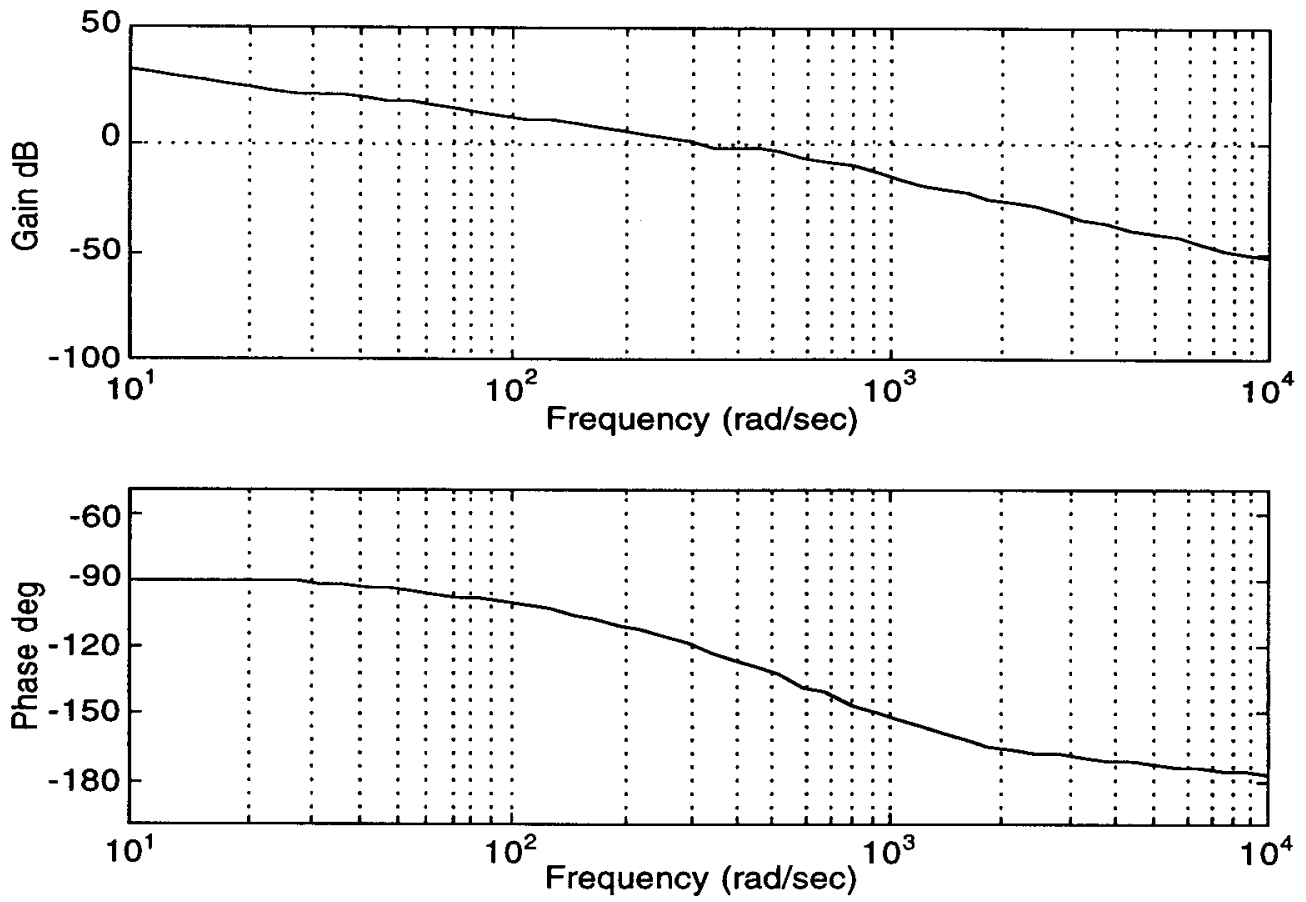

Figure 6.4 Open loop return ratio (L) Bode plot of system to be matched
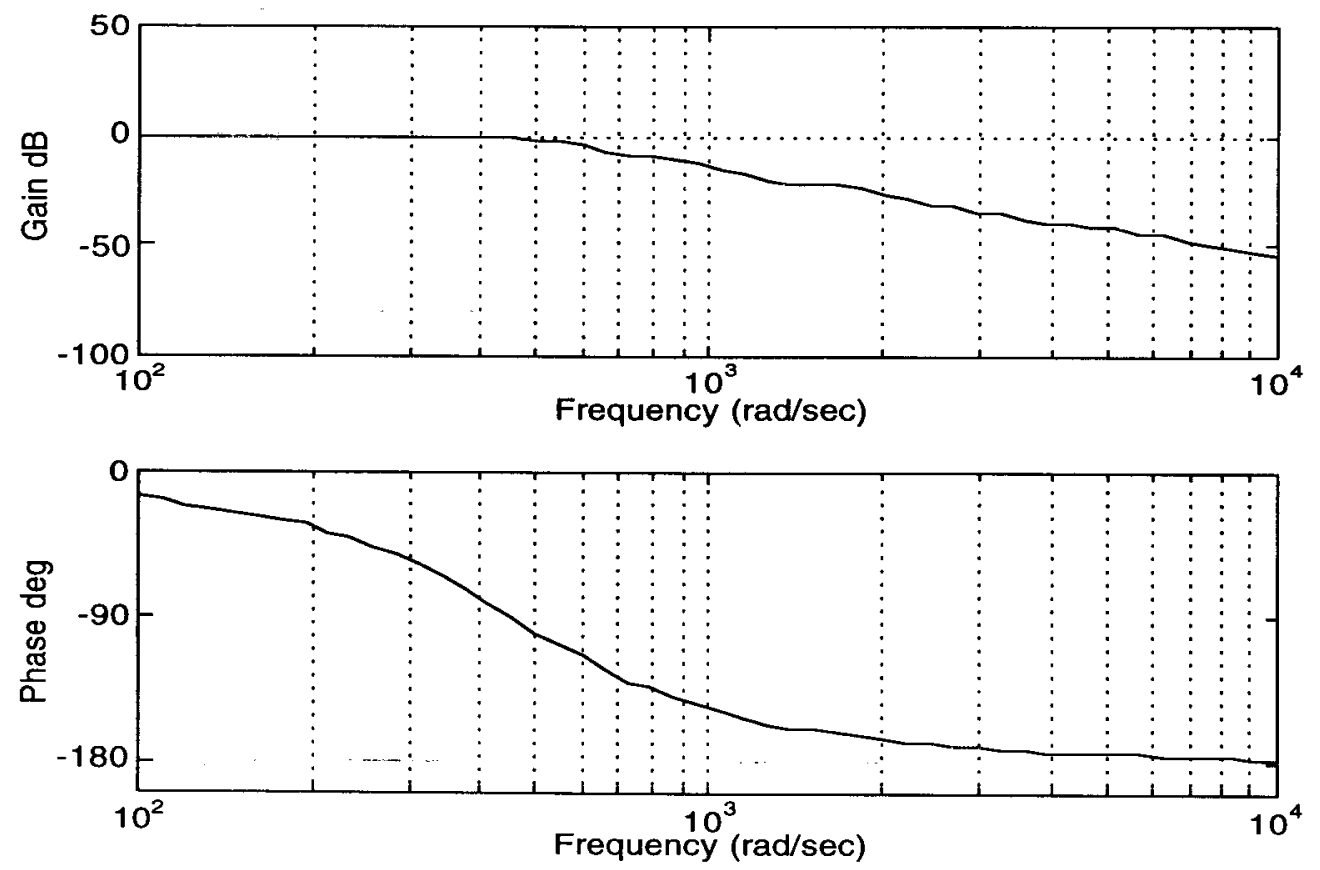

Figure 6.5 Bode plot of matching system complimentary sensitivity $(\mathrm{T})$ function 

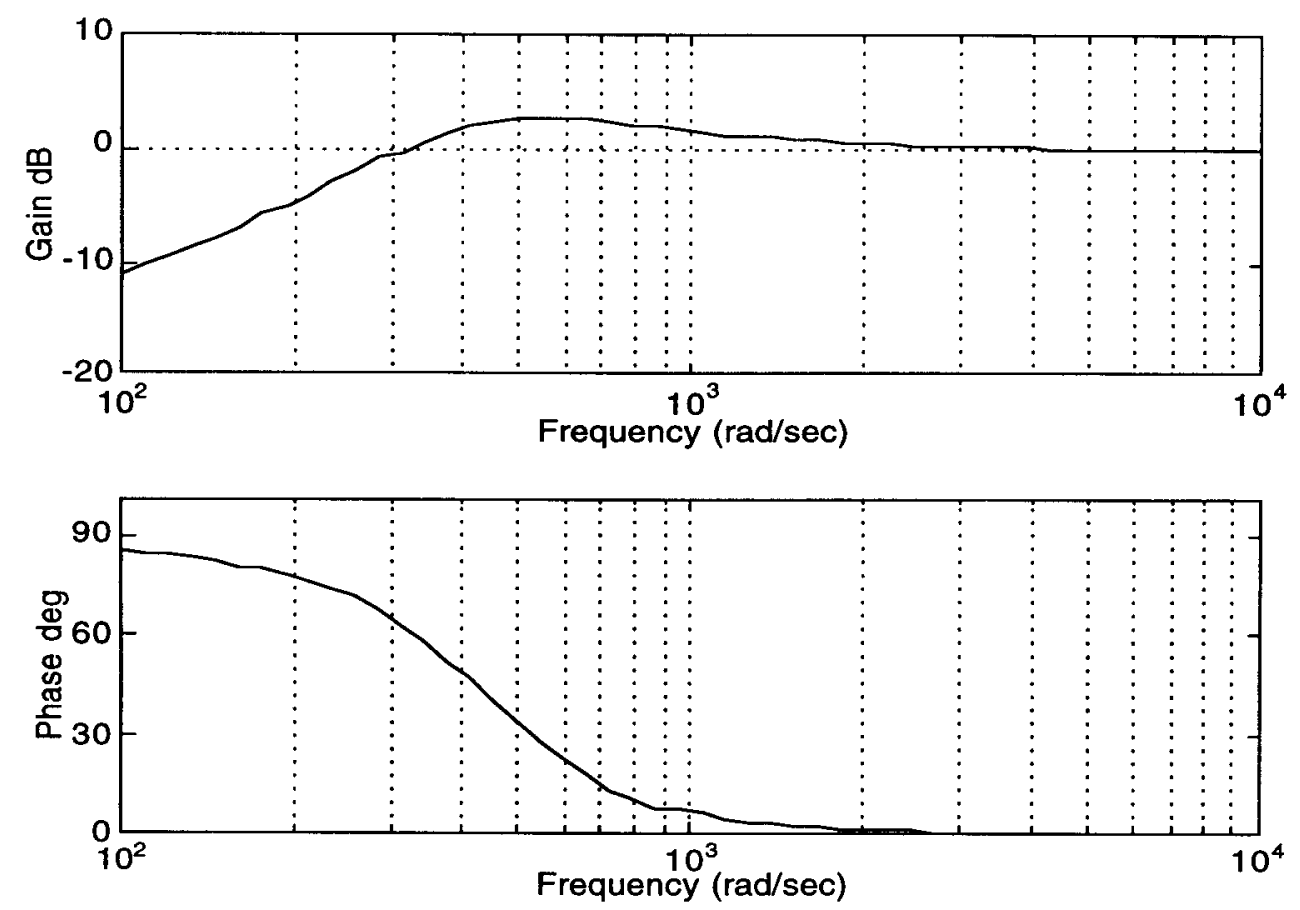

Figure 6.6 Bode plot of matching system sensitivity (S) function

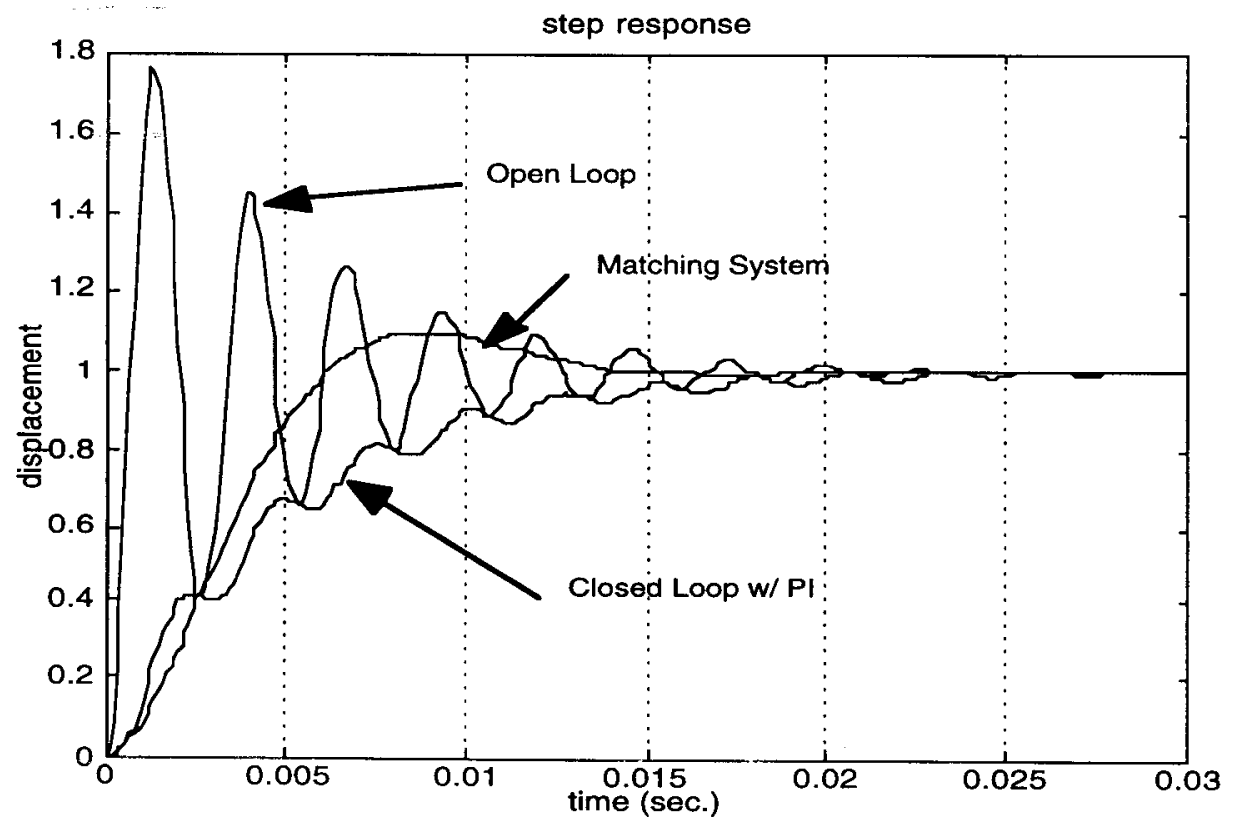

Figure 6.7 Step response for open loop, closed loop and matching system 

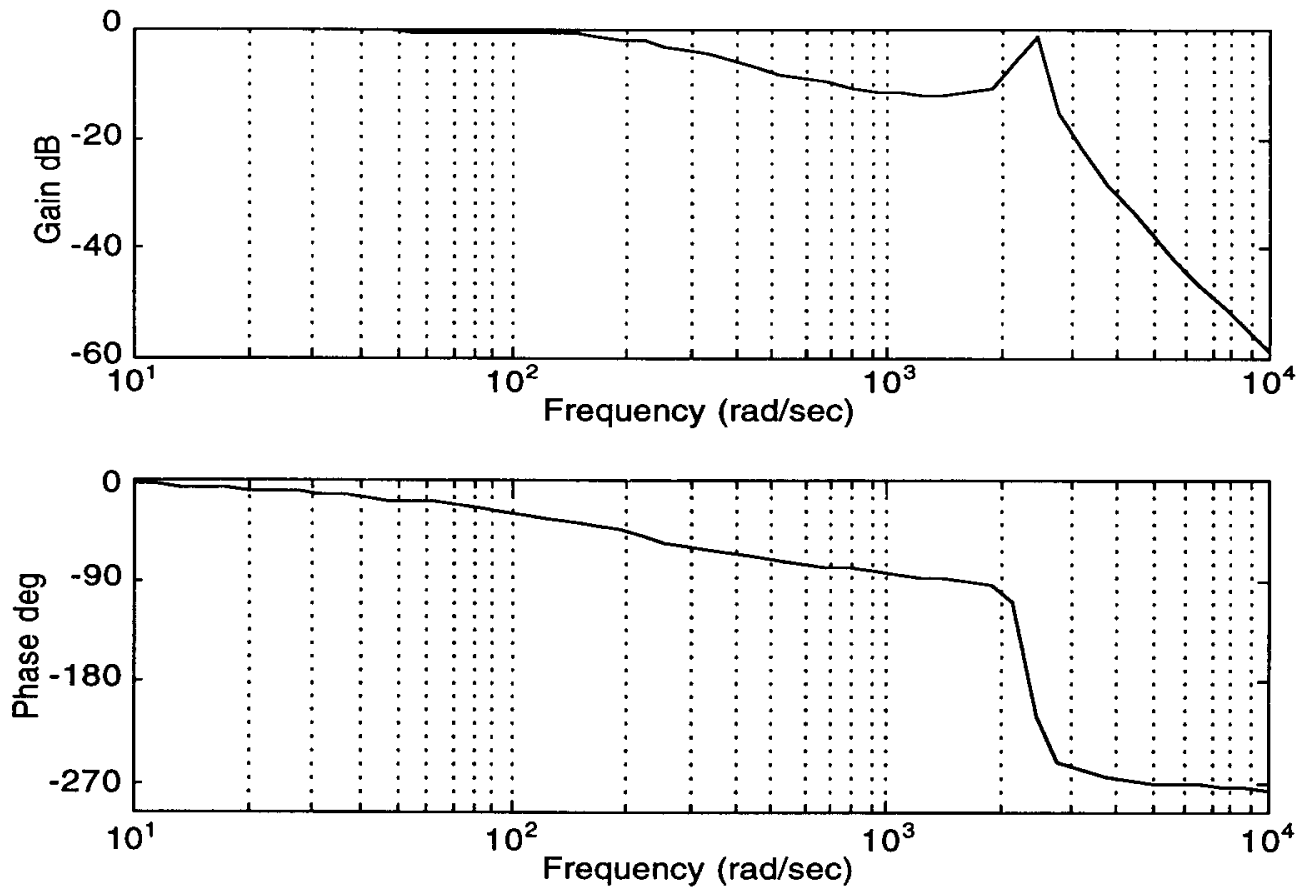

Figure 6.8 Bode plot of complimentary sensitivity (T) of closed loop system
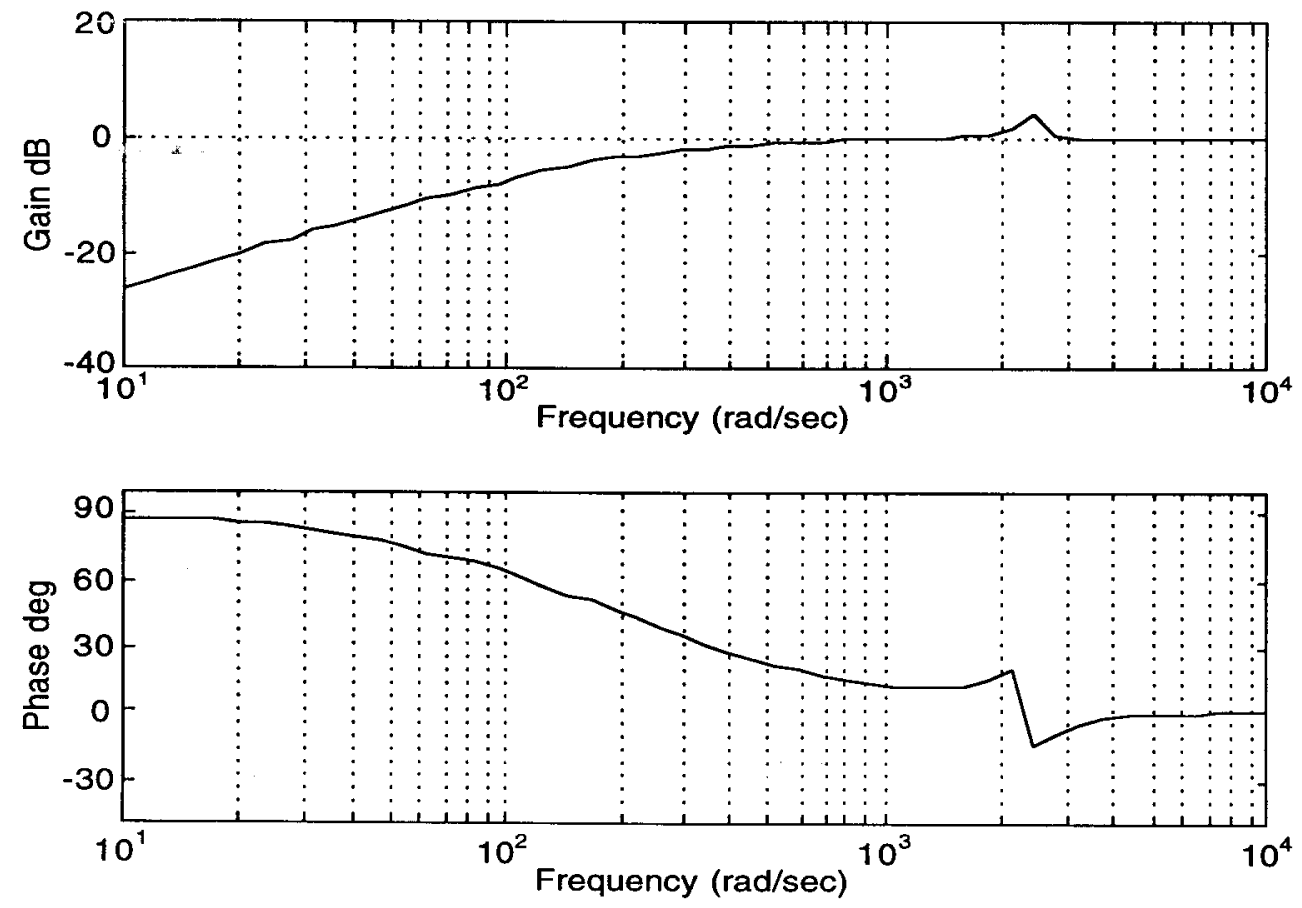

Figure 6.9 Bode plot of sensitivity (S) of closed loop system 

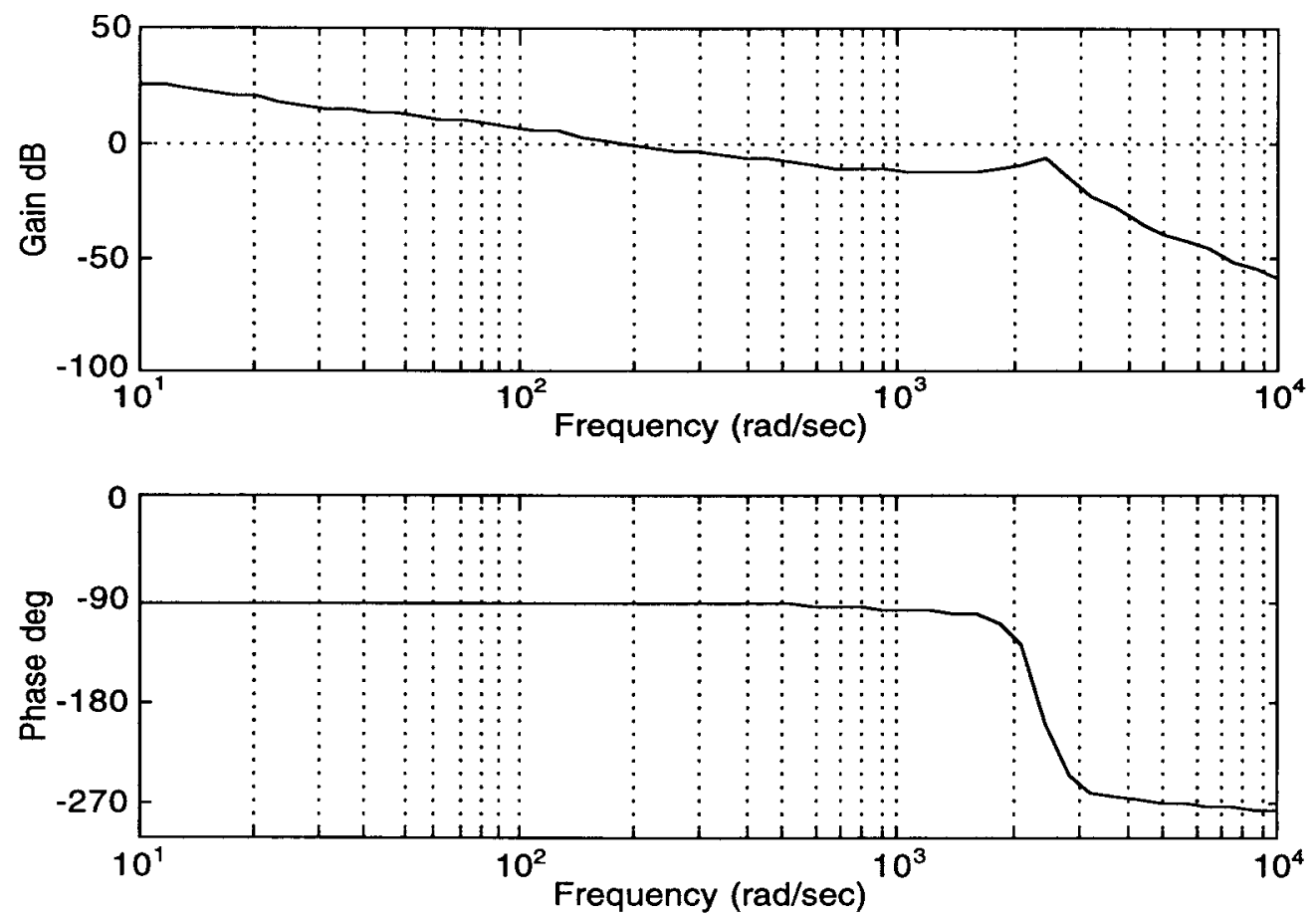

Figure 6.10 Bode plot of return ratio (L) of closed loop system

\subsection{MIMO Proportional-Integral (PI) Control Simulation}

The purpose of this section is to develop a MIMO controller based on a traditional proportional-integral approach. The controller was developed utilizing the dynamic model from the previous chapter. All simulations utilized the Matlab programming language. First, the control development focused on deriving the proportional-integral MIMO controller in state space form. The general PI controller can be written as

$$
G_{c}=K_{p}+\frac{K_{i}}{s},
$$

and in state space form the matrices are written as

$$
K_{i}=C^{T} B
$$


and

$$
K_{p}=D
$$

where,

$$
\begin{aligned}
& A=0(\mathrm{nxn}), \\
& B=I_{r}(\mathrm{nxr}), \\
& C^{T}=k_{i} I_{n}(\mathrm{mxn}), \text { and } \\
& D=k_{p} I_{r}(\mathrm{mxr}) .
\end{aligned}
$$

These equations are derived by manipulating the state space equations (Brewer, 1995).

First, set

$$
A=0
$$

and then substitute into the state space equations giving

$$
\begin{aligned}
& \dot{\bar{x}}=B \bar{u}, \\
& \dot{\bar{x}}=s \bar{x} \text { and } \\
& \bar{y}=C^{T} \bar{x}+D \bar{u} .
\end{aligned}
$$

Now, combining Equations 6.20 through 6.22 , results in

$$
\grave{y}=\left(C^{T} \dot{B}\left(\frac{1}{s}\right)+D\right) \bar{u}
$$

and 


$$
G_{c}=C^{T} B\left(\frac{1}{s}\right)+D
$$

By comparing Equations 6.24 and 6.16 it is proven that Equations 6.17 and 6.18 are the proportional-integral MIMO controller matrices.

With the controller state space equations developed and utilizing the dynamic model developed in Chapter 5, the equations were coded using the Matlab programming language and are shown in Appendix E. With the control model complete, $k_{p}$ and $k_{i}$ were adjusted to achieve the fastest response without significantly sacrificing overshoot and stability. Stability was confirmed by evaluating the open loop and closed loop system eigenvalues. After iterating through various gains, the following values were chosen, $k_{p}=0.5$ and $k_{i}=4500$. Figures 6.11 and 6.12 are open loop frequency and step responses, respectively, and are repeated from Chapter 5 for comparison purposes.

Figure 6.13 shows the frequency response of the return ratio, which has the typical PI controller $-20 \mathrm{~dB} /$ decade slope. In general, this is a reasonable return ratio since it has "high" gain at low frequencies for rejecting disturbances and "low" gain at high frequencies for attenuating noise. These results support the frequency response plots of sensitivity (S) and complimentary sensitivity (T) shown in Figures 6.14 and 6.15, respectively. Lastly, a closed loop step response is shown in Figure 6.16. It is noted that the results are very similar to the results for the closed loop SISO controller, as shown in Figure 6.7.

Next, the robustness of the PI controller design was evaluated. As stated earlier, robustness is defined as the ability to maintain the properties of stability and performance as the plant parameters vary, relative to the nominal design.

To evaluate the controllers robustness, the plant parameters were varied and the system stability and performance was evaluated. Defining the plant model developed in Chapter 5 as nominal, the PI controller was developed. Using this controller, the plant model parameters were modified and simulations completed. The plant model changes 
included reducing the damping by a factor of 2 and increasing the mass by a factor of 2 . The open loop response are depicted in Figure 6.17 and 6.18. Figures 6.19 through 6.21 depict the frequency response plots for the return ratio $(\mathrm{L})$, complimentary sensitivity $(\mathrm{T})$, and sensitivity (S), respectively, for the PI controller. In general the results are slightly degraded as compared to the nominal plant performance. Figure 6.22 depicts the step response for the PI controller, which is unstable. These results indicate the lack of robustness for this typical PI controller design. To overcome this shortfall, the next section will cover the development of a controller utilizing the $\mathrm{H}_{\infty}$ controller design process.

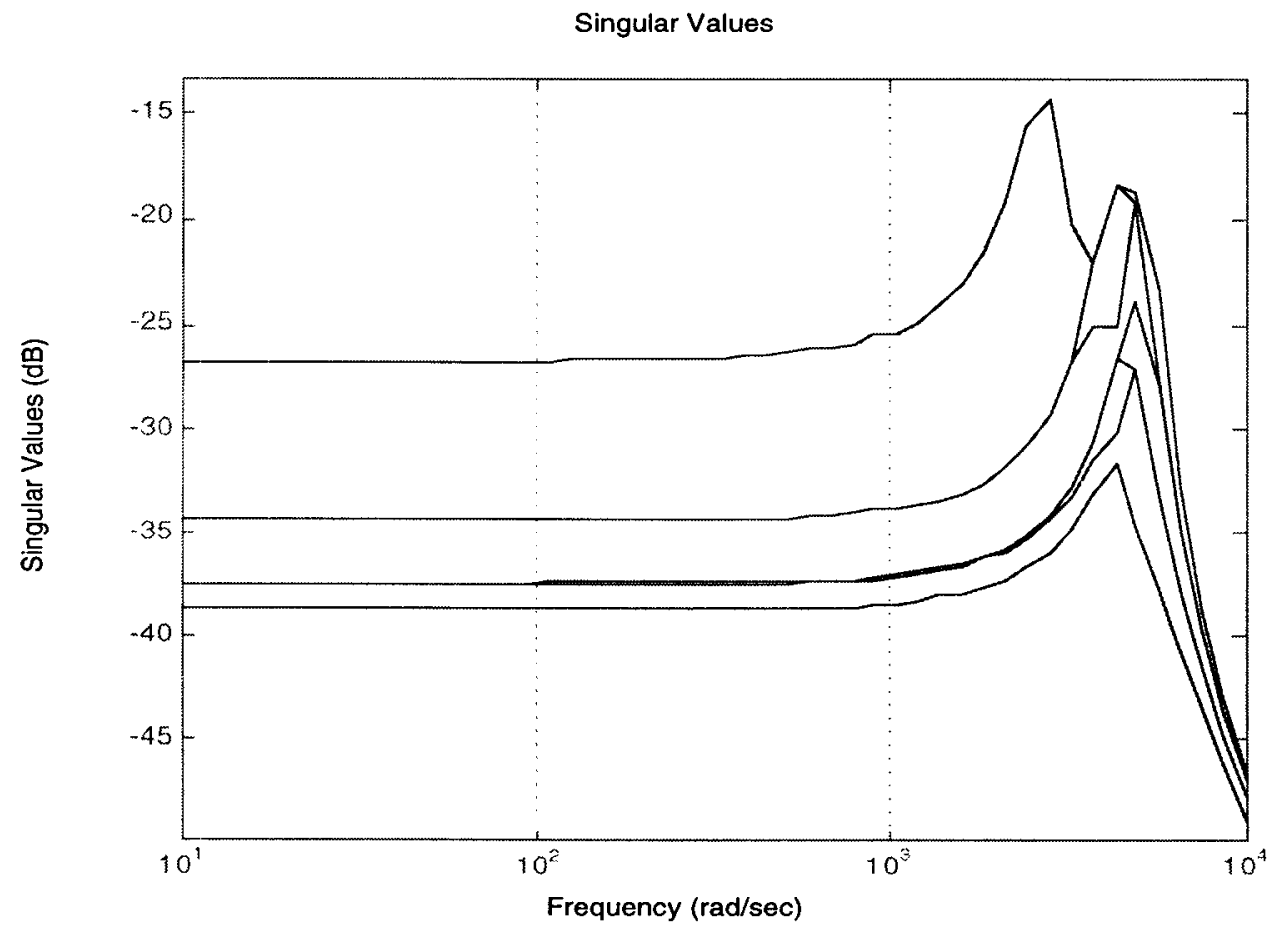

Figure 6.11 Open loop frequency plot 


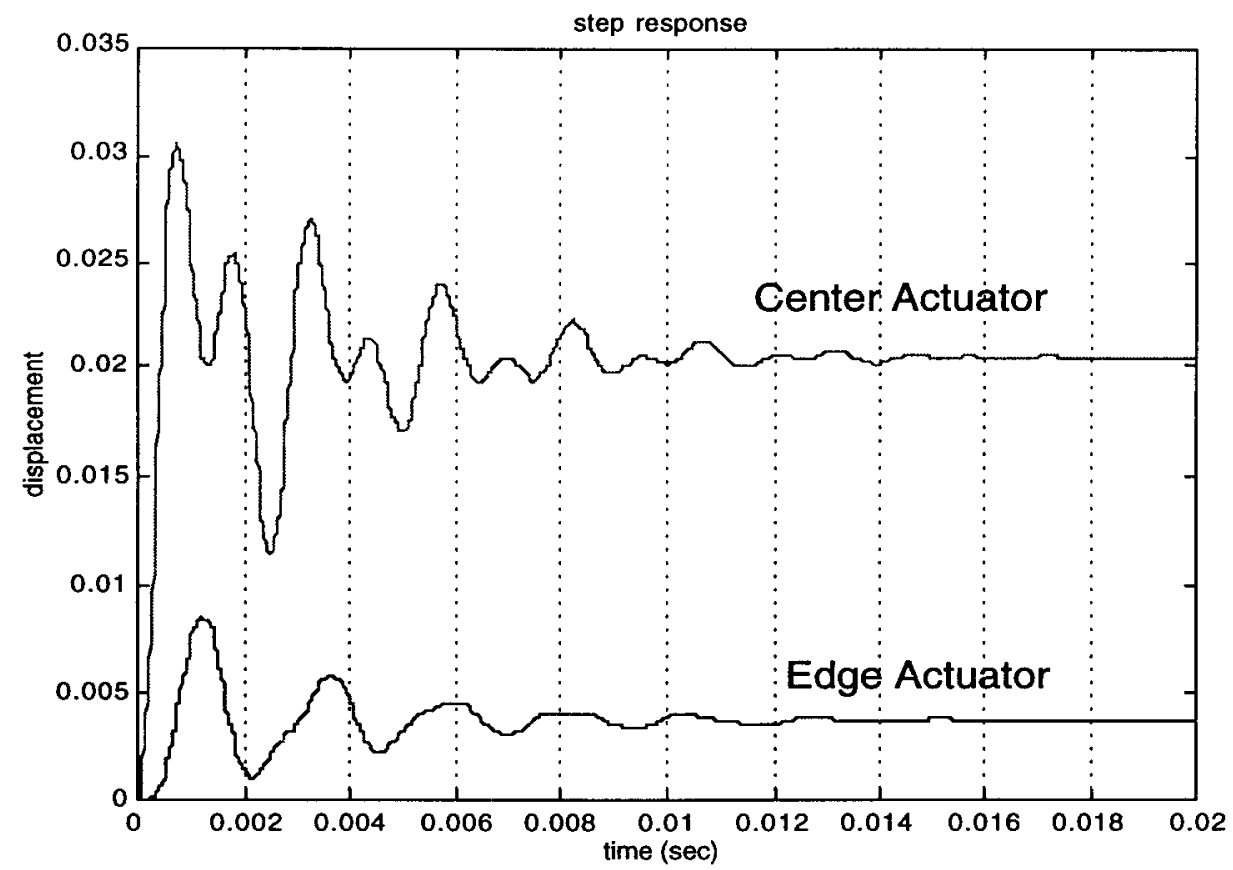

Figure 6.12 Open loop step response

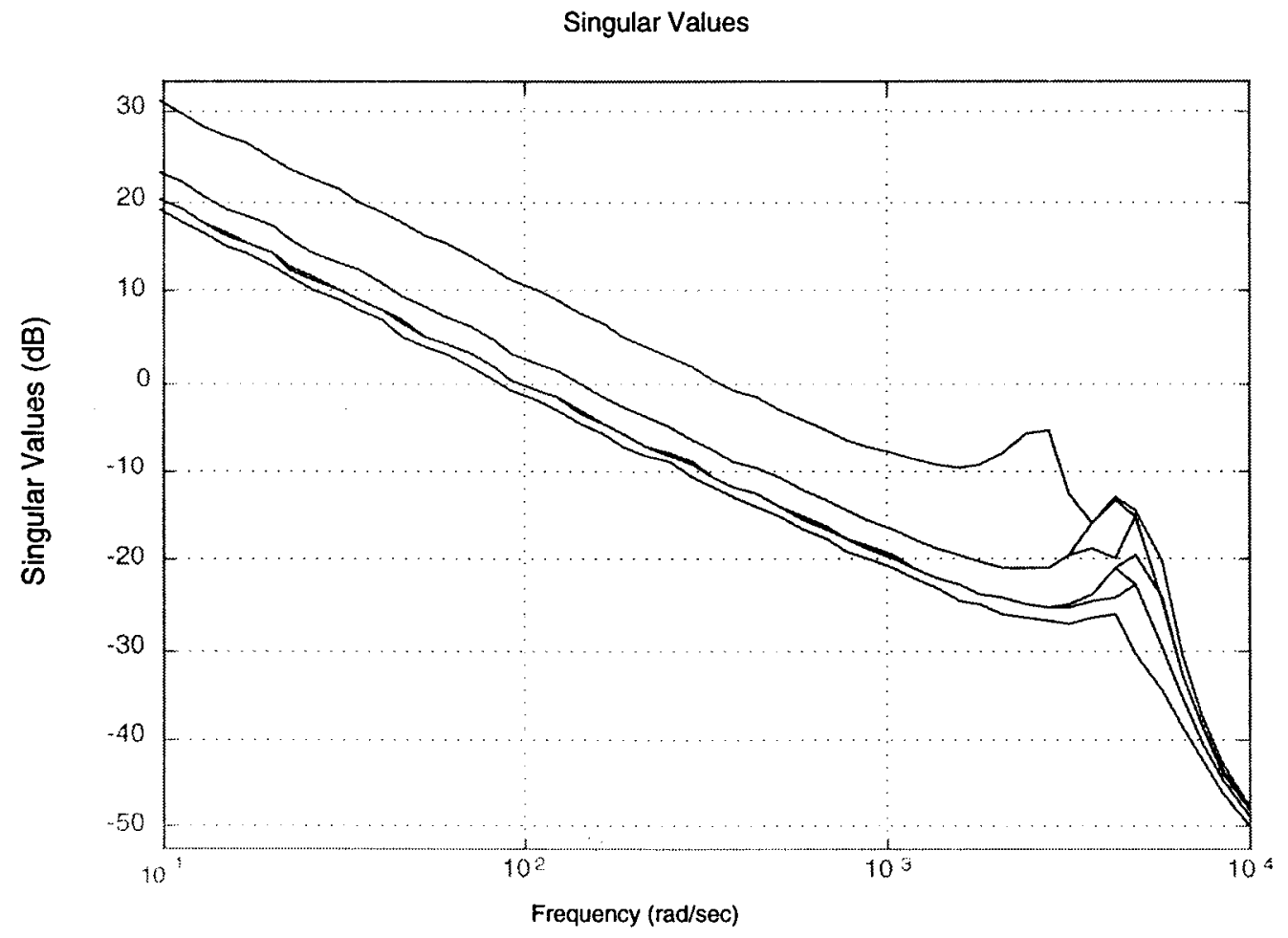

Figure 6.13 Frequency plot of return ratio $\left(\mathrm{L}_{\mathrm{y}}\right)$ using PI control 


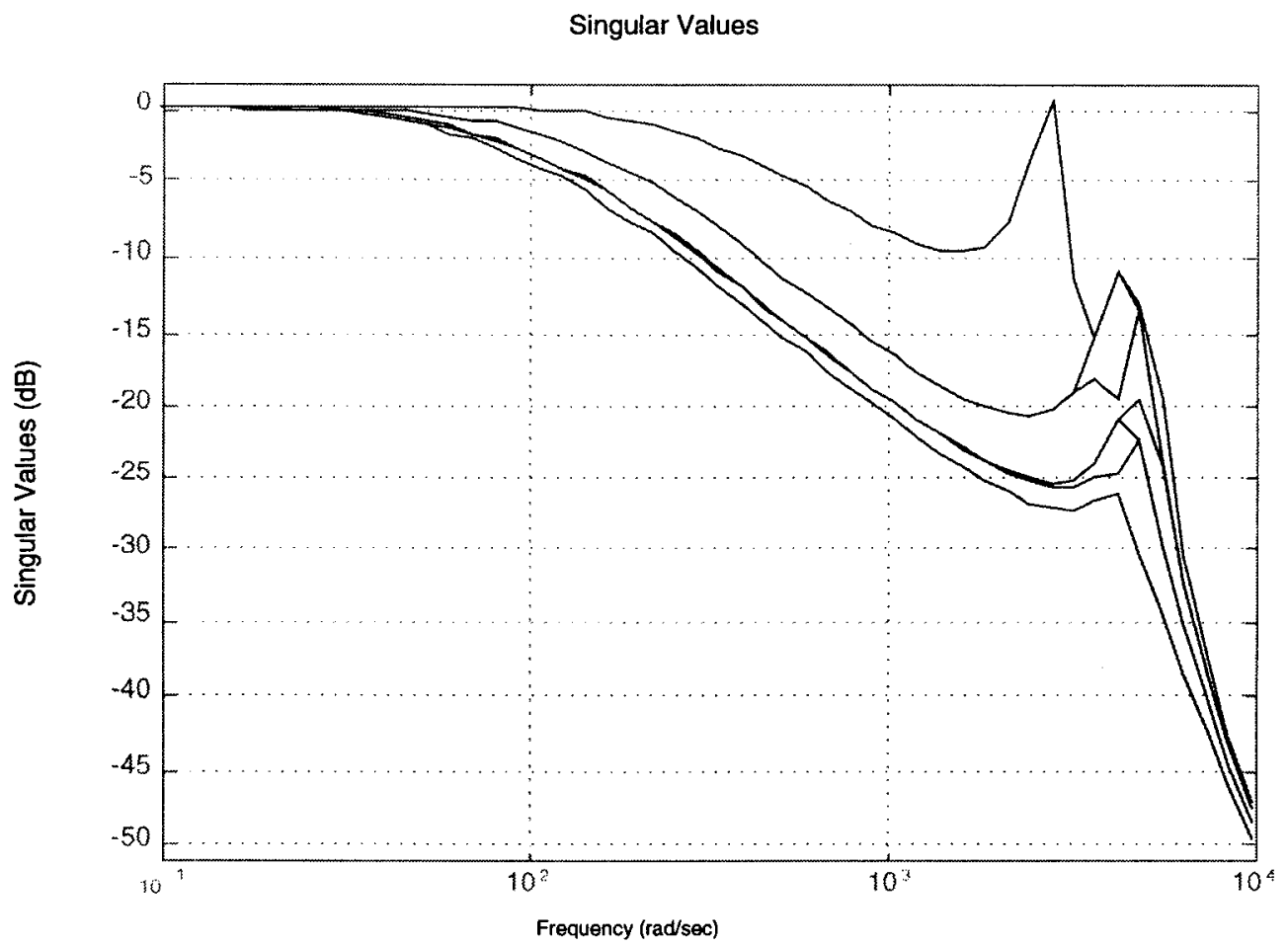

Figure 6.14 Frequency plot of complimentary sensitivity $\left(\mathrm{T}_{\mathrm{y}}\right)$ using PI control

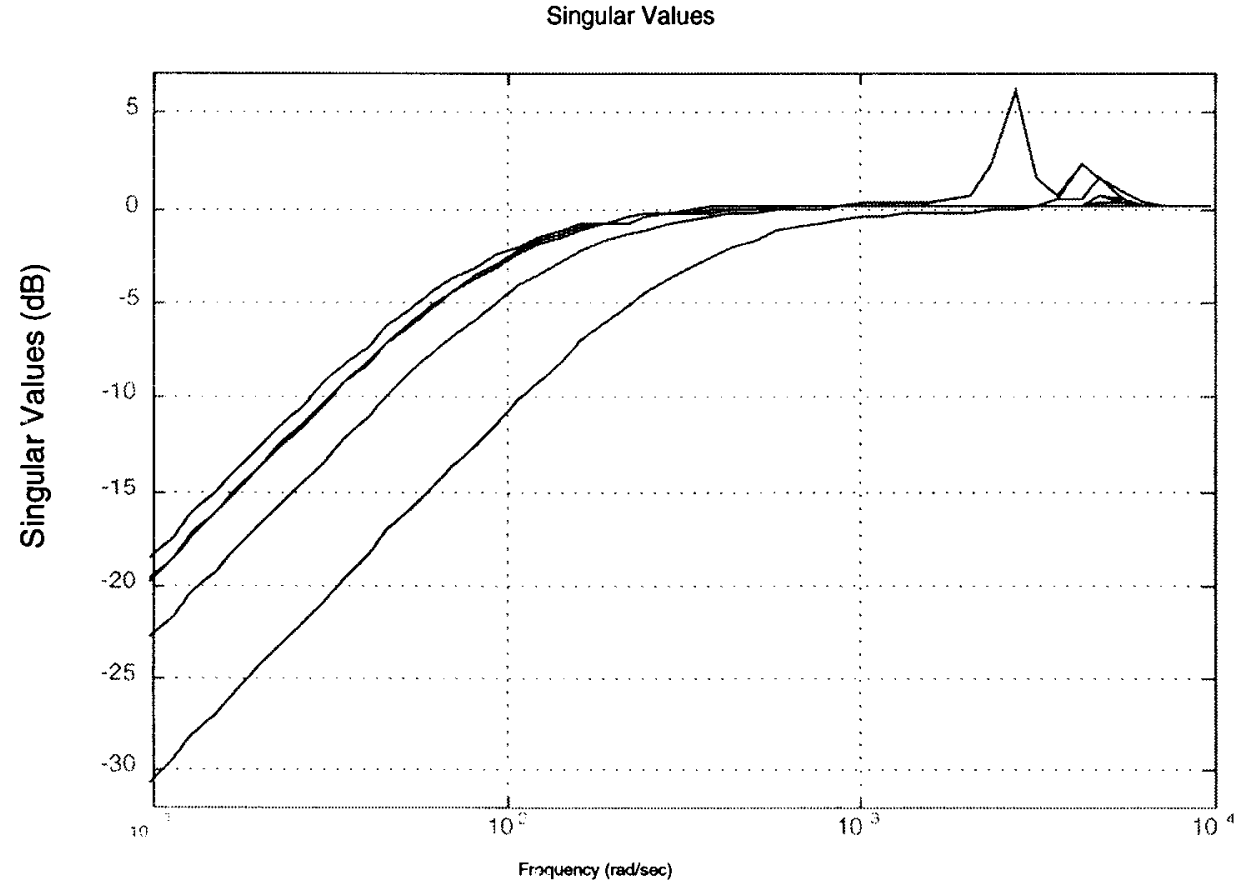

Figure 6.15 Frequency plot of sensitivity $\left(S_{y}\right)$ using PI control 


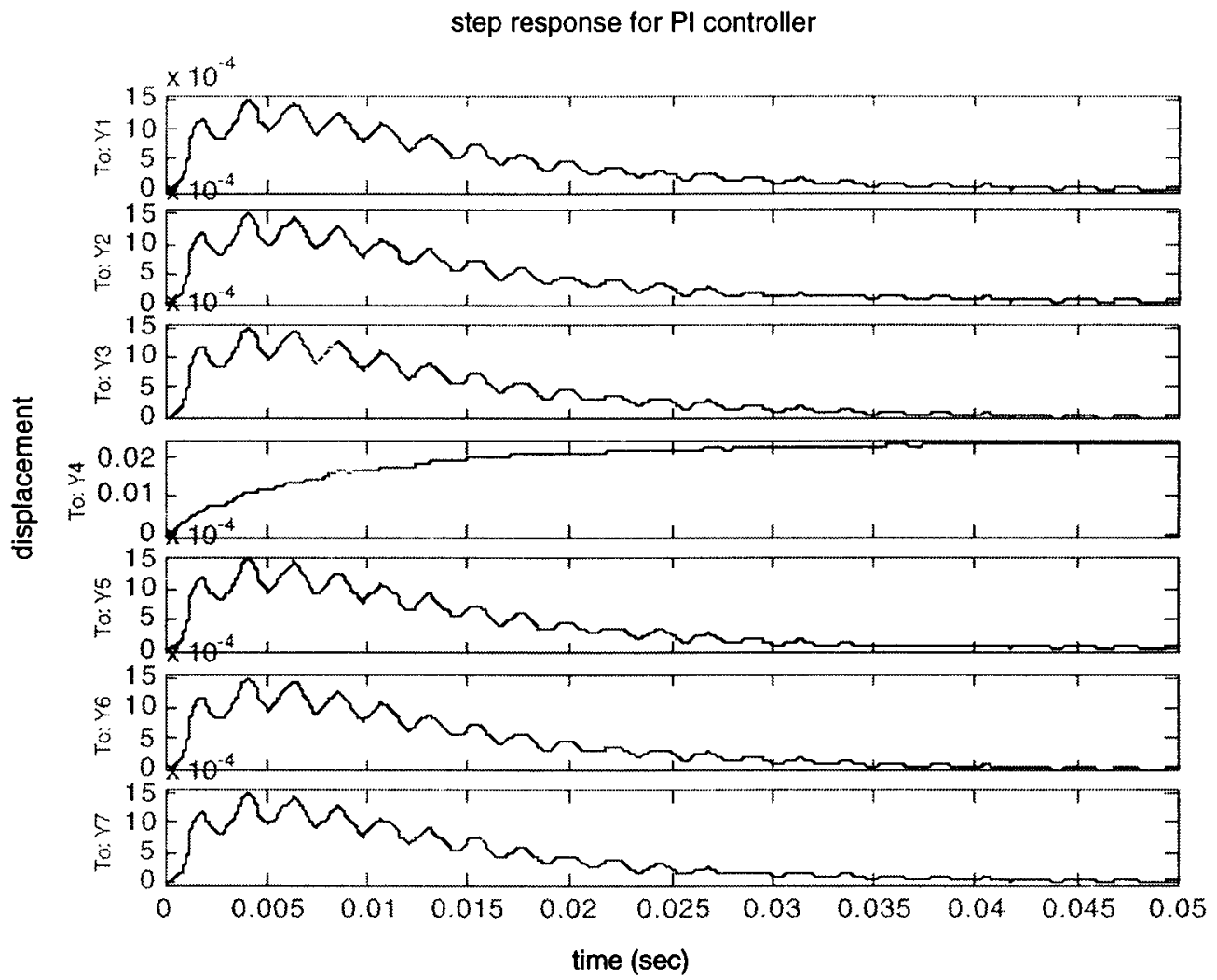

Figure 6.16 Close loop step response for PI controller 


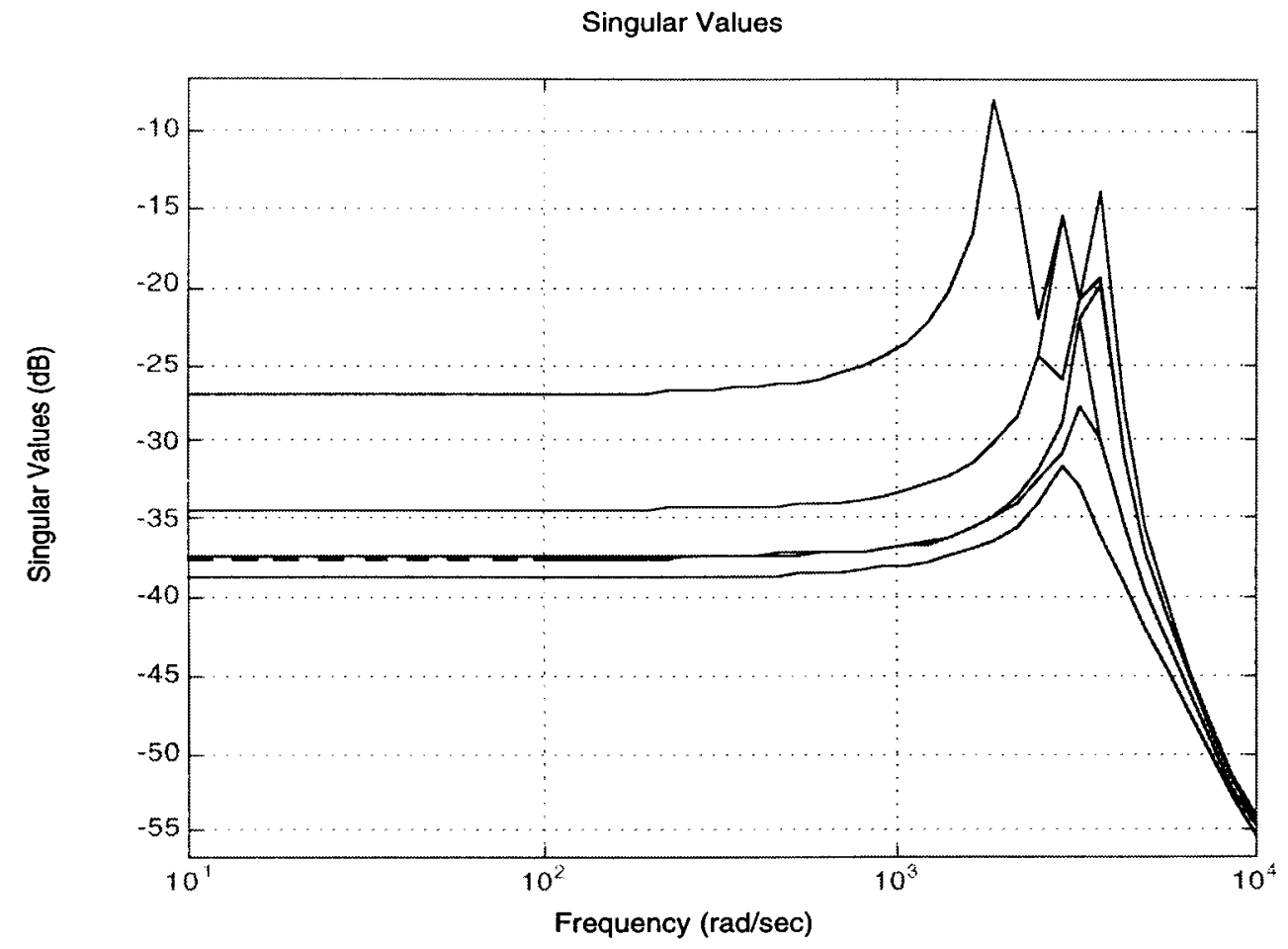

Figure 6.17 Open loop singular value plot of modified plant model

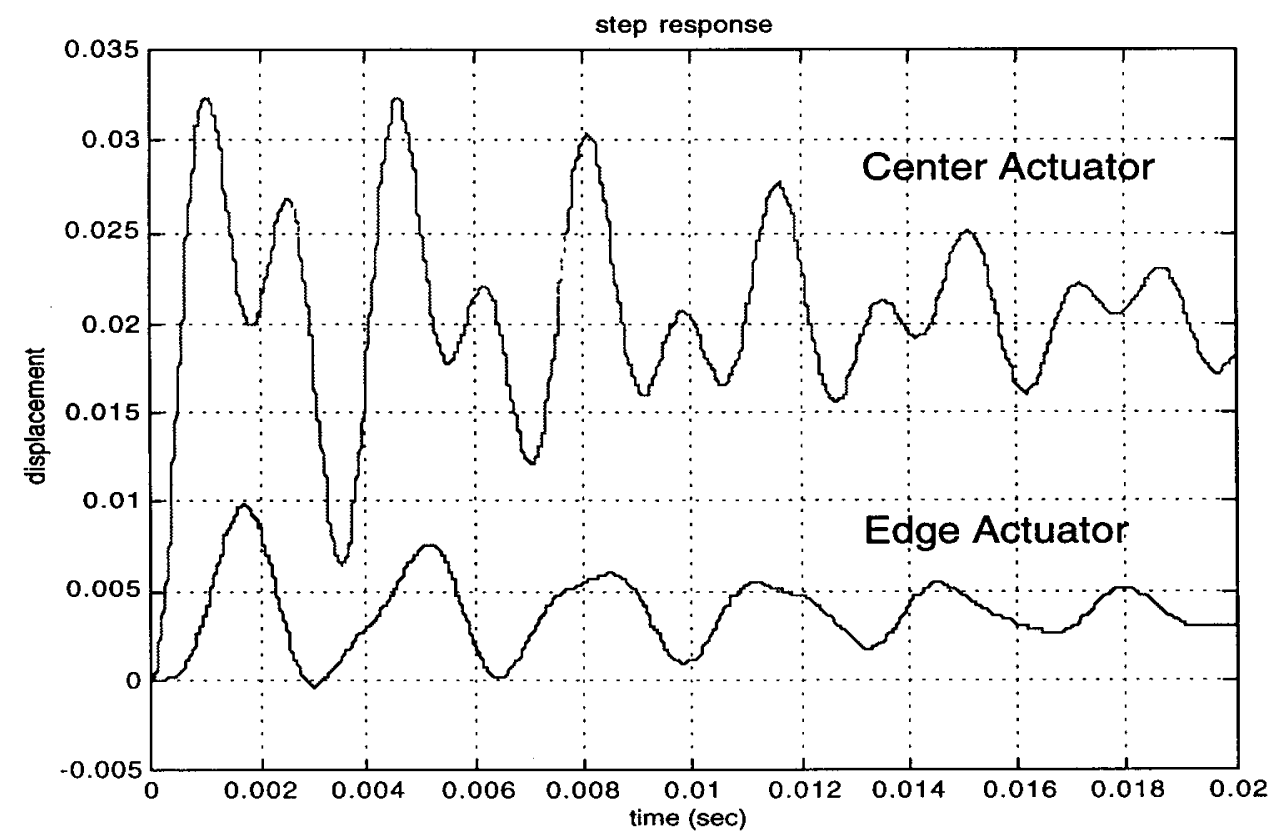

Figure 6.18 Open loop step response of modified plant model 


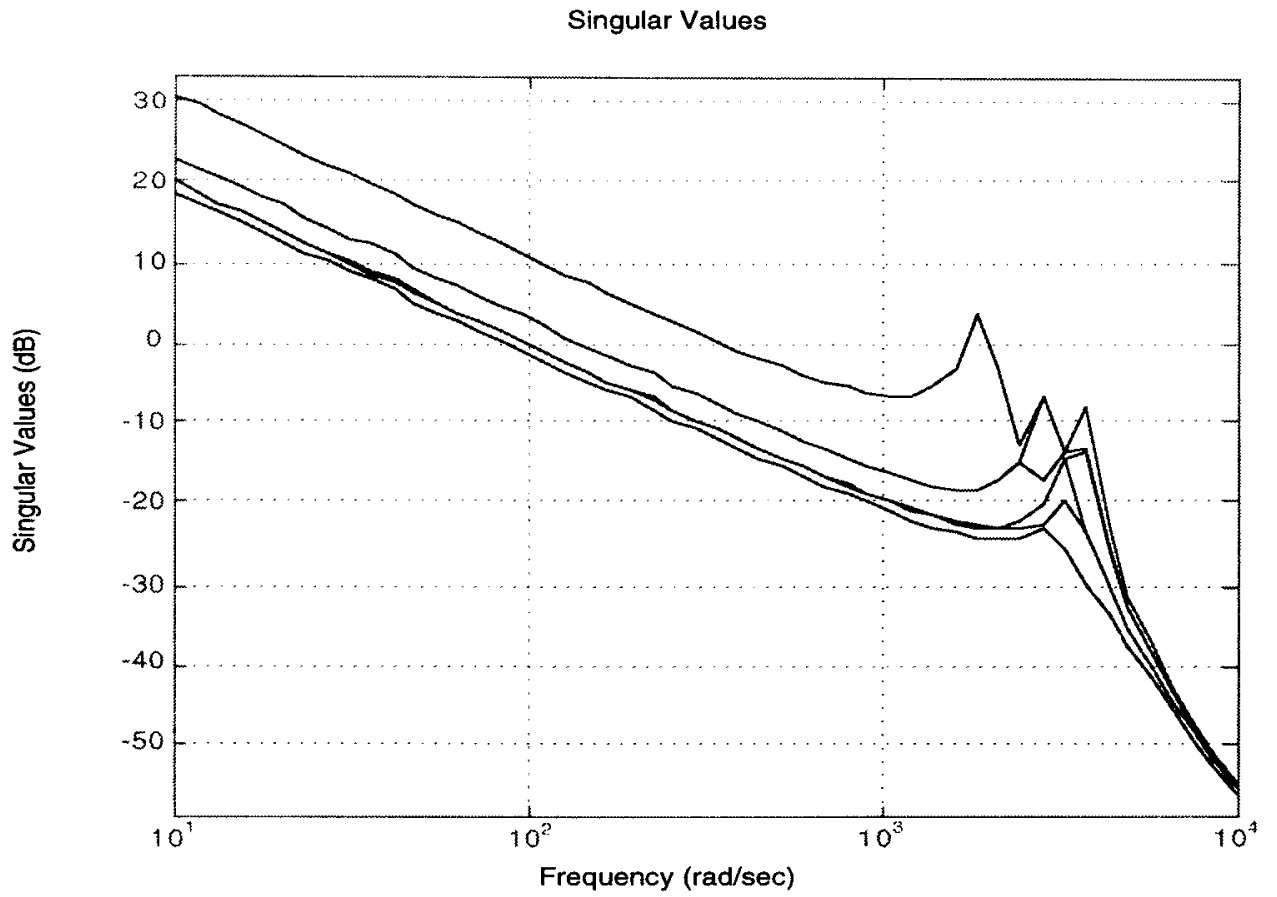

Figure 6.19 Frequency response of $\left(\mathrm{L}_{\mathrm{y}}\right)$ using PI controller

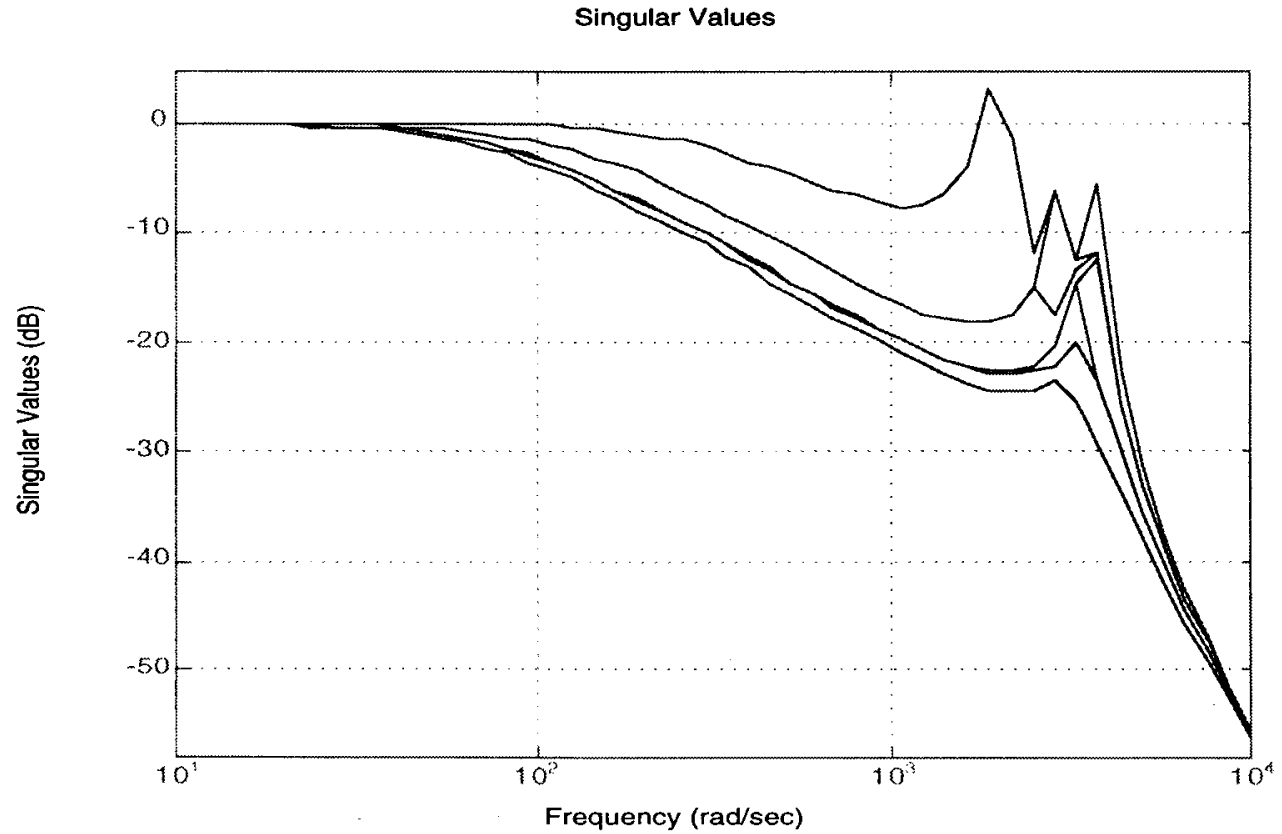

Figure 6.20 Frequency response of complimentary sensitivity $\left(\mathrm{T}_{\mathrm{y}}\right)$ using PI controller 


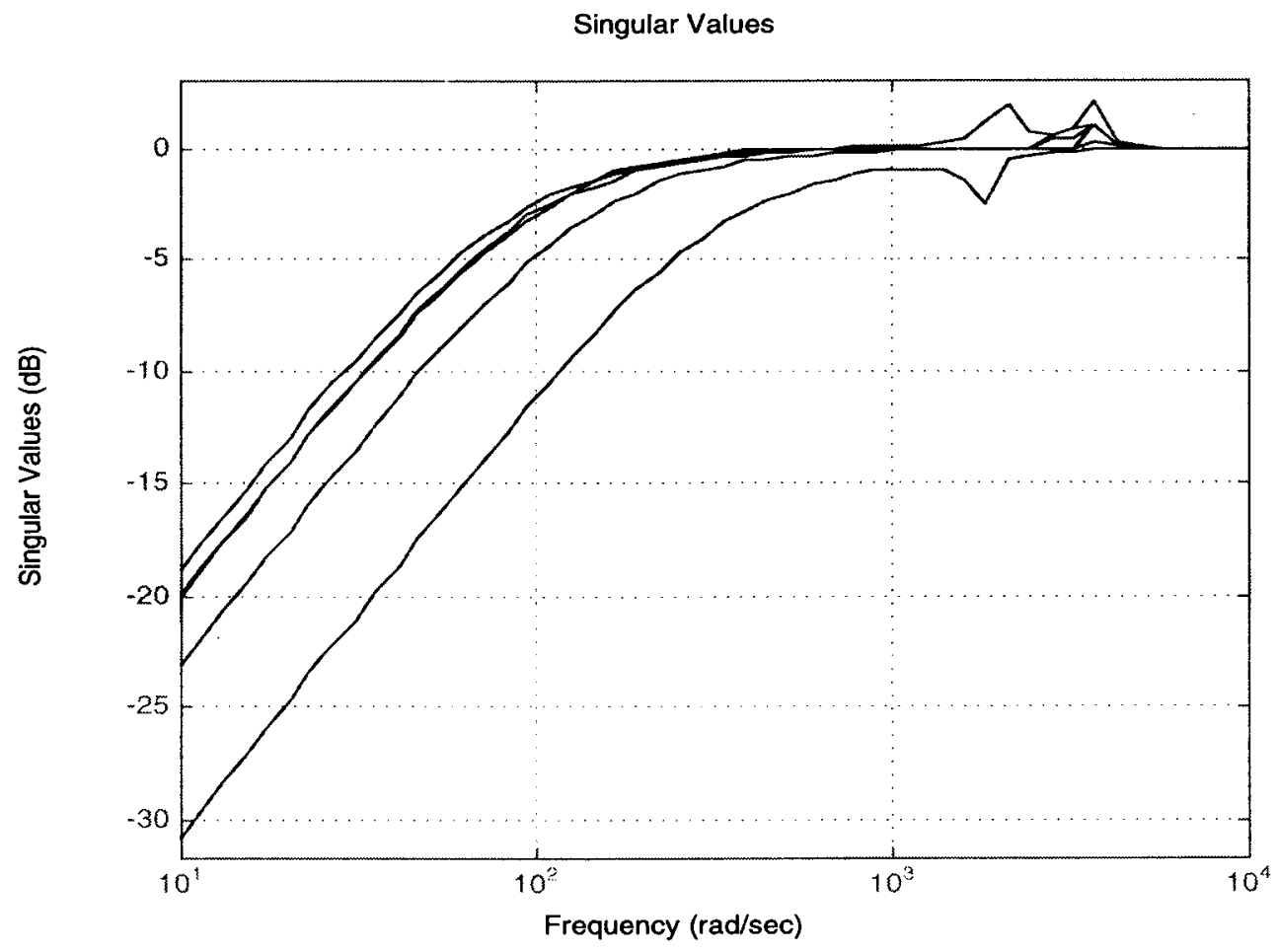

Figure 6.21 Frequency response of sensitivity $\left(S_{y}\right)$ using PI controller 
step response for PI controller

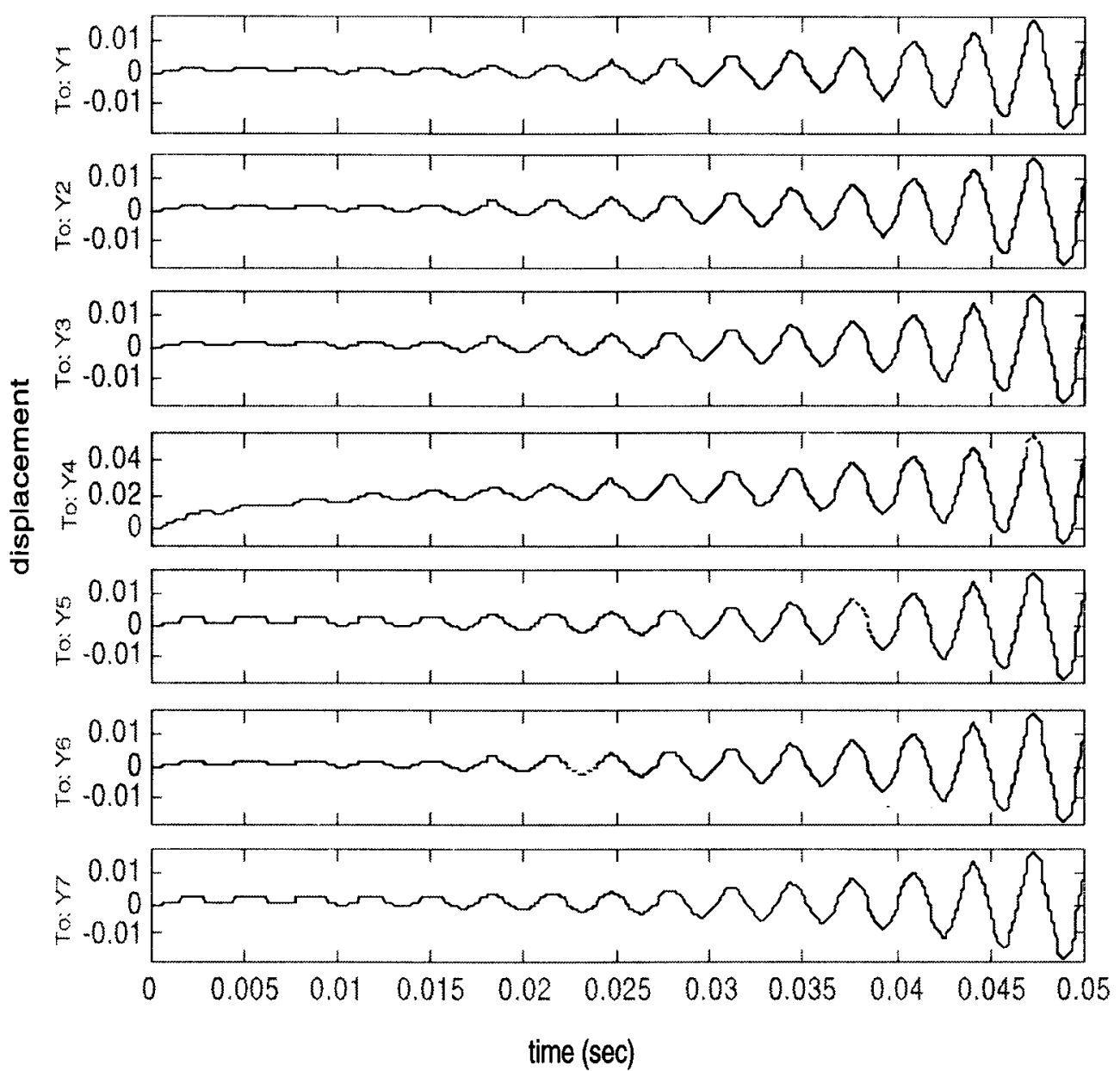

Figure 6.22 Step response using PI controller

\subsection{MIMO $\mathrm{H}_{\infty}$ Control Simulation}

In this section the $\mathrm{H}_{\infty}$ controller design process will be described. The goal in robust multivariable control system design is to synthesize a controller which maintains system response and error signals within tolerances despite the effects of uncertainty on the system. Uncertainty, can take many forms, however, the most significant are 
noise/disturbances and modeling errors. Therefore, uncertainty is by far the most significant issue in controller design. $\mathrm{H}_{\infty}$ theory provides a direct reliable procedure for synthesizing a controller which optimally satisfies singular value specifications. However, before reviewing the design theory, some general controller properties are stated (Safonov, et al, 1989; Safonov, et al, 1992):

1. The $\mathrm{H}_{\infty}$ optimal control cost function $T_{z w}$ is all pass, i.e., $\bar{\sigma}\left[T_{z w}\right]=1$ for all $\omega \in R$,

2. An $\mathrm{H}_{\infty}$ "sub-optimal" controller has order equal to that of the augmented plant ( $\mathrm{n}$ state). An $\mathrm{H}_{\infty}$ optimal controller can be computed having at most (n-1) states,

3. In any weighted mixed sensitivity problem formulation, the $\mathrm{H}_{\infty}$ controller always cancels the stable poles of the plant with its transmission zeros, and

4. In the weighted mixed sensitivity problem formulation, any unstable pole of the plant inside the specified control bandwidth will be shifted approximately to its $\overline{j \omega}-$ axis mirror image once the feedback loop is closed with an $\mathrm{H}_{\infty}$ controller.

The $\mathrm{H}_{\infty}$ augmented plant is shown in Figure 6.23 with $\mathrm{G}_{\mathrm{A}}$ described by

$$
\begin{aligned}
& \dot{x}=A x+B_{1} w+B_{2} u, \\
& z=C_{1} x+D_{11} w+D_{12} u, \text { and } \\
& y=C_{2} x+D_{21} w+D_{22} u .
\end{aligned}
$$




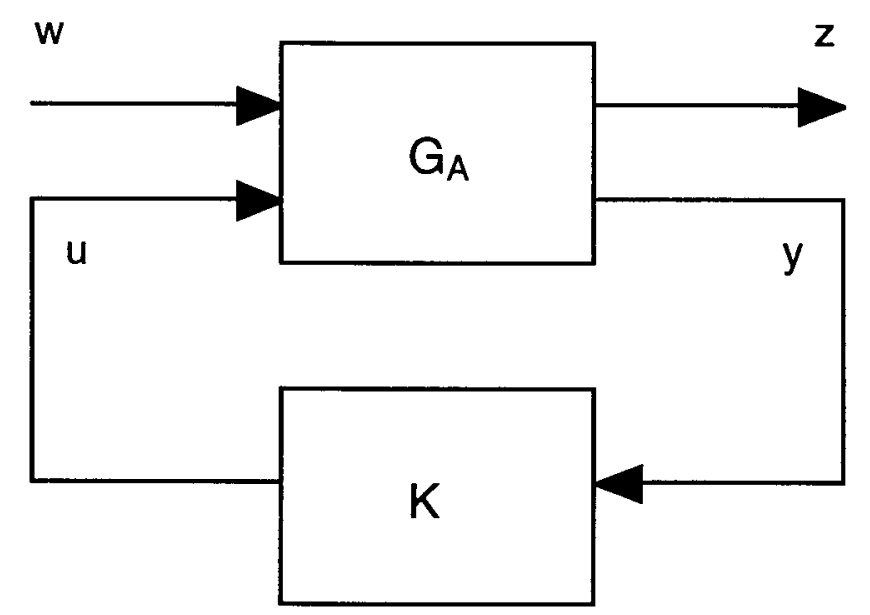

Figure 6.23 Augmented plant and controller for $\mathrm{H}_{\infty}$ design

The variable $w$ represents process and sensor noise, $z$ is the regulated variable and $y$ is the input to the controller. Figure 6.23 is also referred to as a linear fractional transformation (LFT) on $\mathrm{K}$, and $\mathrm{G}_{\mathrm{A}}$ is called the coefficient matrix for the LFT (Doyle, et al, 1989). The $\mathrm{H}_{\infty}$ design process will minimize $\left\|T_{z w}\right\|_{\infty}$ by the appropriate choice of …......

controller $\mathrm{K}$, where

$$
T_{z w}=\left[\begin{array}{l}
W_{1} S \\
W_{2} K S \\
W_{3} T
\end{array}\right]
$$

The weighting functions $\mathrm{W}_{1}, \mathrm{~W}_{2}$ and $\mathrm{W}_{3}$ are chosen to reflect the design objectives. The original plant $G_{p}$ is augmented with $W_{1}, W_{2}$ and $W_{3}$ to give $G_{A}$. The weighting functions are not completely arbitrary, as they are subject to some constraints. The $\mathrm{H}_{\infty}$ theory gives four conditions (constraints) for the existence of a solution to the standard $\mathrm{H}_{\infty}$ control problem and are stated as follows:

1. $D_{11}$ small enough. There must exist a constant feedback control law $F(s)=$ "constant matrix" such that the closed loop D matrix satisfies $\bar{\sigma}(D)<1$, 
2. Control Riccati $P \geq 0$. The $\mathrm{H}_{\infty}$ full-state feedback control Riccati equation must have real, positive semidefinite solution $P$,

3. Observer Riccati $S \geq 0$. The Riccati equation associated with the observer dual of the $\mathrm{H}_{\infty}$ full-state feedback control problem must have real, positive semidefinite solution $S$, and

4. $\lambda_{\max }(P S)<1$. The greatest eigenvalue of the product of the two Riccati equation solutions must be less than one.

These four conditions must hold for there to exist a feedback control law which solves the standard $\mathrm{H}_{\infty}$ control problem (Safonov, et al, 1992).

Utilizing the Matlab Robust Control Toolbox, the above $\mathrm{H}_{\infty}$ design methodology was applied to the same system as that used in the PI controller design. After many iterations and meeting the design constraints, the weighting functions $\mathrm{W}_{1}, \mathrm{~W}_{2}$ and $\mathrm{W}_{3}$ were chosen as

$$
\begin{aligned}
& \mathrm{W}_{1}=\left[\begin{array}{ccccccc}
\frac{s+10000}{10000 s+1} & 0 & 0 & 0 & 0 & 0 & 0 \\
0 & \frac{s+10000}{10000 s+1} & 0 & 0 & 0 & 0 & 0 \\
0 & 0 & . & 0 & 0 & 0 & 0 \\
0 & 0 & 0 & . & 0 & 0 & 0 \\
0 & 0 & 0 & 0 & . & 0 & 0 \\
0 & 0 & 0 & 0 & 0 & . & 0 \\
0 & 0 & 0 & 0 & 0 & 0 & \frac{s+10000}{10000 s+1}
\end{array}\right], \\
& \mathrm{W}_{2}=[] \text {, and }
\end{aligned}
$$




$$
\mathrm{W}_{3}=\left[\begin{array}{ccccccc}
\frac{s^{3}}{100000000000} & 0 & 0 & 0 & 0 & 0 & 0 \\
0 & \frac{s^{3}}{100000000000} & 0 & 0 & 0 & 0 & 0 \\
0 & 0 & . & 0 & 0 & 0 & 0 \\
0 & 0 & 0 & . & 0 & 0 & 0 \\
0 & 0 & 0 & 0 & . & 0 & 0 \\
0 & 0 & 0 & 0 & 0 & . & 0 \\
0 & 0 & 0 & 0 & 0 & 0 & \frac{s^{3}}{100000000000}
\end{array}\right] .
$$

Next, the $\mathrm{H}_{\infty}$ controller performance will be discussed. Figure 6.24, the open loop return ratio singular value plot, has characteristics of a good design. The low frequency gain is "high" which will help reject disturbances and the high frequency gain is "low" which will help attenuate noise influences. Figures 6.25 and 6.26 are the closed loop complimentary sensitivity (T) and sensitivity (S) singular value plots, respectively. Both plots show good characteristics, as is expected from the return ratio plot. Figure 6.26, the sensitivity plot, does show some negative characteristics in the $200-900 \mathrm{~Hz}$ range where the disturbance is amplified a maximum of $5 \mathrm{~dB}$. Figure 6.27 shows the closed loop step response for an input applied to the center actuator. The strong decoupling between the output channels for an input to the center actuator is noted.

As completed for the PI design, the robustness of the $\mathrm{H}_{\infty}$ controller design was evaluated using the same modified model. Figures 6.28 through 6.30 depict the frequency response plots for the return ratio $(\mathrm{L})$, complimentary sensitivity $(\mathrm{T})$, and sensitivity $(\mathrm{S})$, respectively. These results are slightly different relative to the nominal plant performance. Figure 6.31 depicts the step response, which shows more coupling than the nominal design, but is clearly stable. 
Comparing the results from both the proportional-integral (PI) design and $\mathrm{H}_{\infty}$ design shows a clear improvement for the $\mathrm{H}_{\infty}$ design. Table 6.1 shows a comparison for various performance parameters. From the time domain perspective, the $H_{\infty}$ design produced a faster rise time and settling time, with only a slight overshoot penalty. Additionally, the effort required by the actuators for both the PI and $\mathrm{H}_{\infty}$ designs is very similar, even though the $\mathrm{H}_{\infty}$ design produces better results. Comparing the frequency domain characteristics, the performance, such as disturbance rejection and noise attenuation, is better for the $H_{\infty}$ design. The $H_{\infty}$ design has approximately $9 \mathrm{~dB}$ more disturbance rejection and $11 \mathrm{~dB}$ more noise attenuation as compared to the PI design. Lastly, for the selected model parameter space, the $\mathrm{H}_{\infty}$ controller proved stable and achieved nominal performance objectives. The PI controller showed reasonable performance characteristics, but was unstable to a step input. Therefore, it can be stated that the $\mathrm{H}_{\infty}$ controller provides robust stability and performance as compared to a standard proportional-integral controller design. 


\begin{tabular}{|c|c|c|}
\hline Parameter & PI Controller & H-inf. Controller \\
\hline Time Domain & & \\
\hline rise time & $0.035 \mathrm{sec}$. & $0.0075 \mathrm{sec}$. \\
\hline settling time & $0.035 \mathrm{sec}$. & $0.015 \mathrm{sec}$. \\
\hline percent overshoot & $0.0 \%$ & $10.0 \%$ \\
\hline Frequency Domain & & $-20 \mathrm{~dB}$ \\
\hline T @ 1000 Hz. & $-9 \mathrm{~dB}$ & $-27 \mathrm{~dB}$ \\
\hline S @ 10 Hz. & $-18 \mathrm{~dB}$ & \\
\hline
\end{tabular}

Table 6.1 Performance comparison of PI and $\mathrm{H}_{\infty}$ controller designs

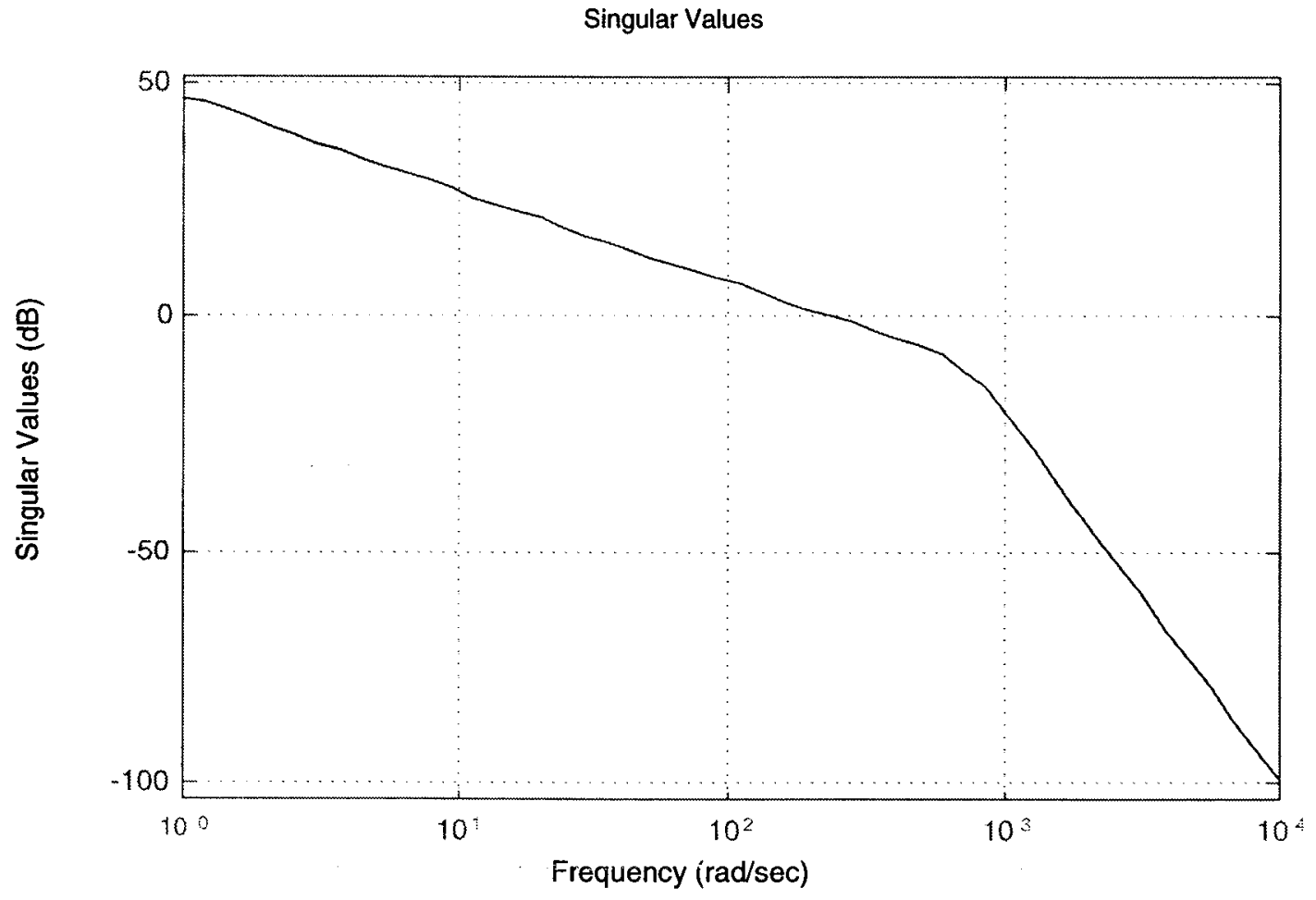

Figure 6.24 Return ratio $\left(\mathrm{L}_{\mathrm{y}}\right)$ frequency plot for $\mathrm{H}_{\infty}$ design 


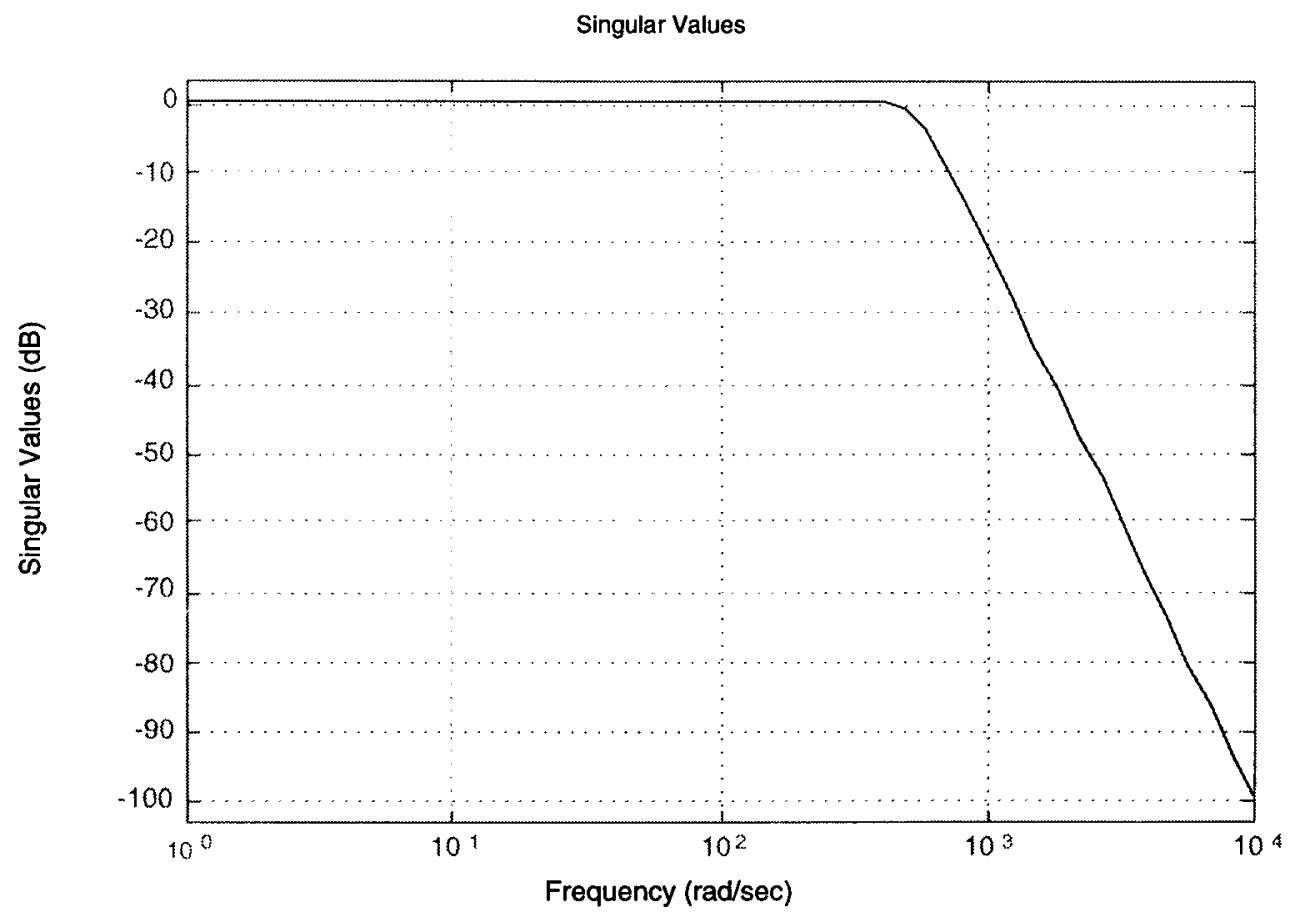

Figure 6.25 Frequency plot of complimentary sensitivity $\left(T_{y}\right)$ for $H_{\infty}$ design

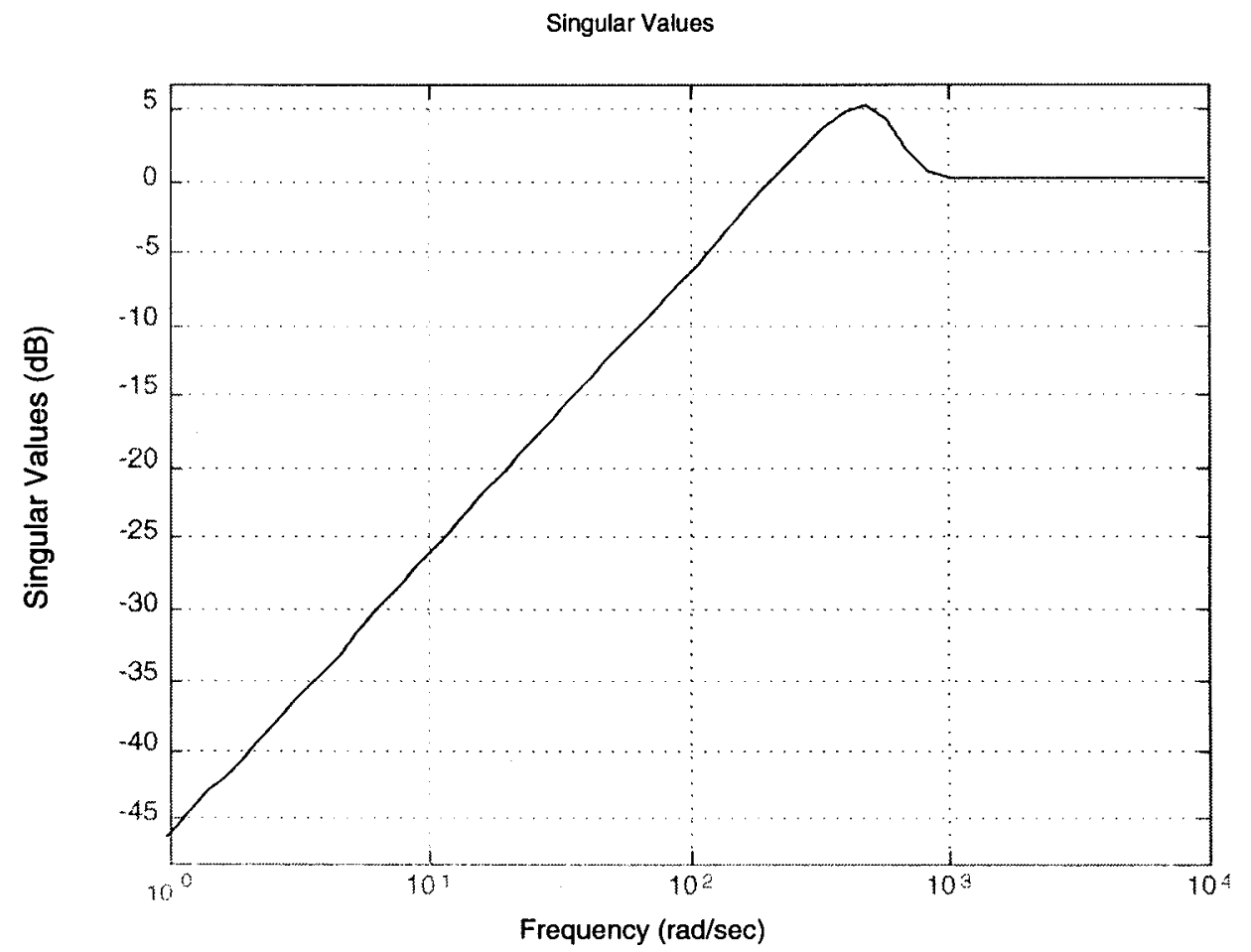

Figure 6.26 Frequency plot of sensitivity $\left(S_{y}\right)$ for $H_{\infty}$ design 


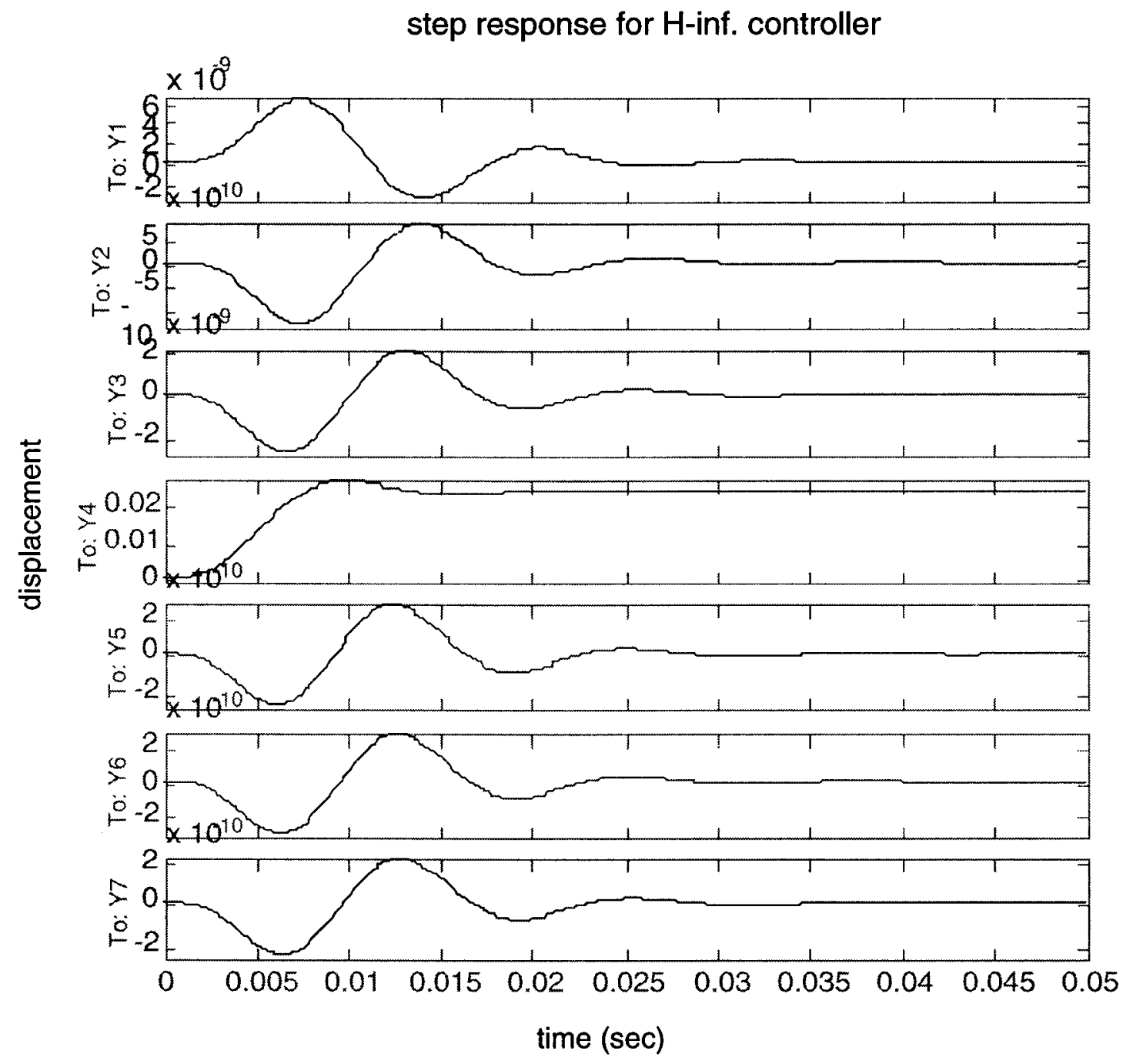

Figure 6.27 Step response applied to the center actuator 


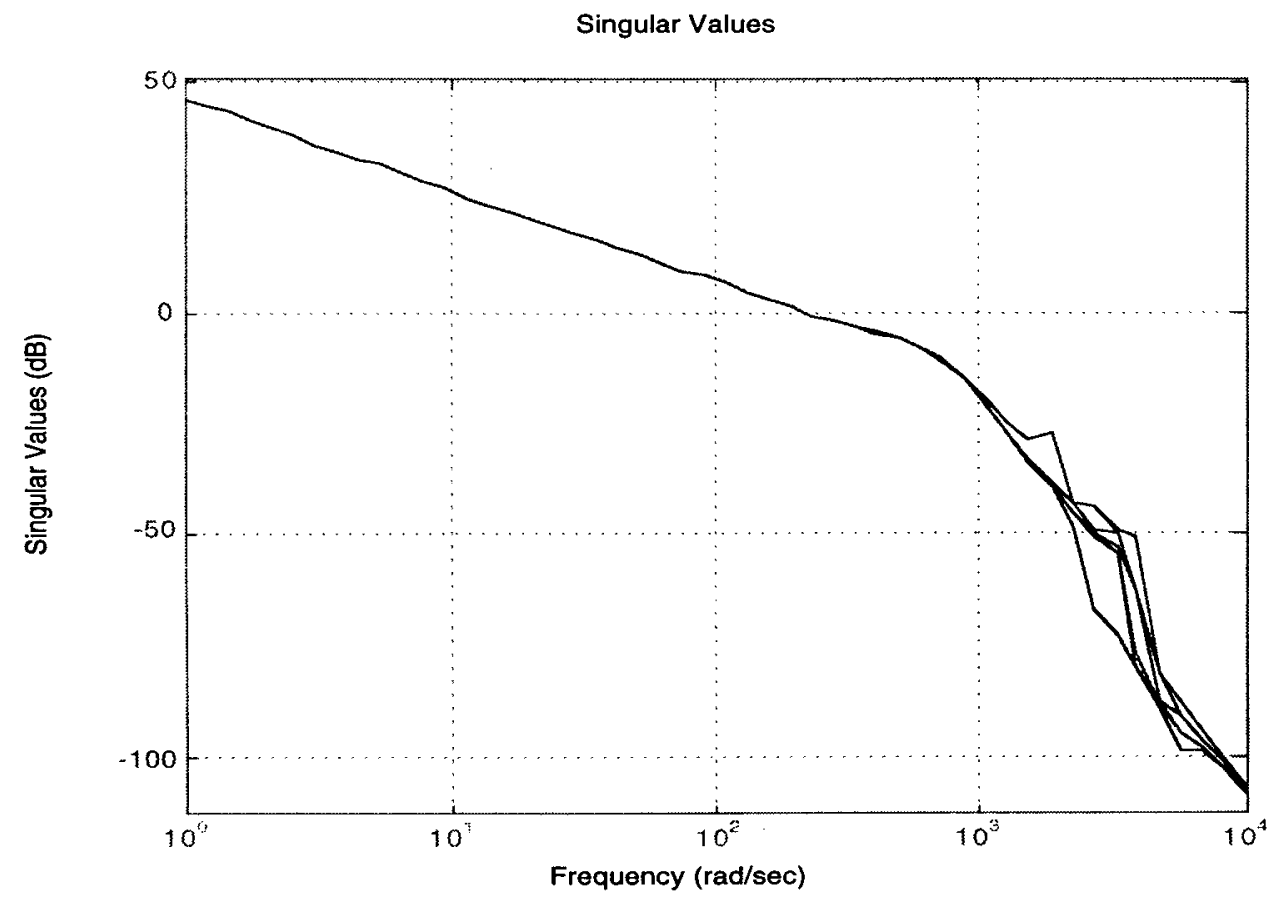

Figure 6.28 Return ration $\left(\mathrm{L}_{\mathrm{y}}\right)$ frequency plot using $\mathrm{H}_{\infty}$ controller

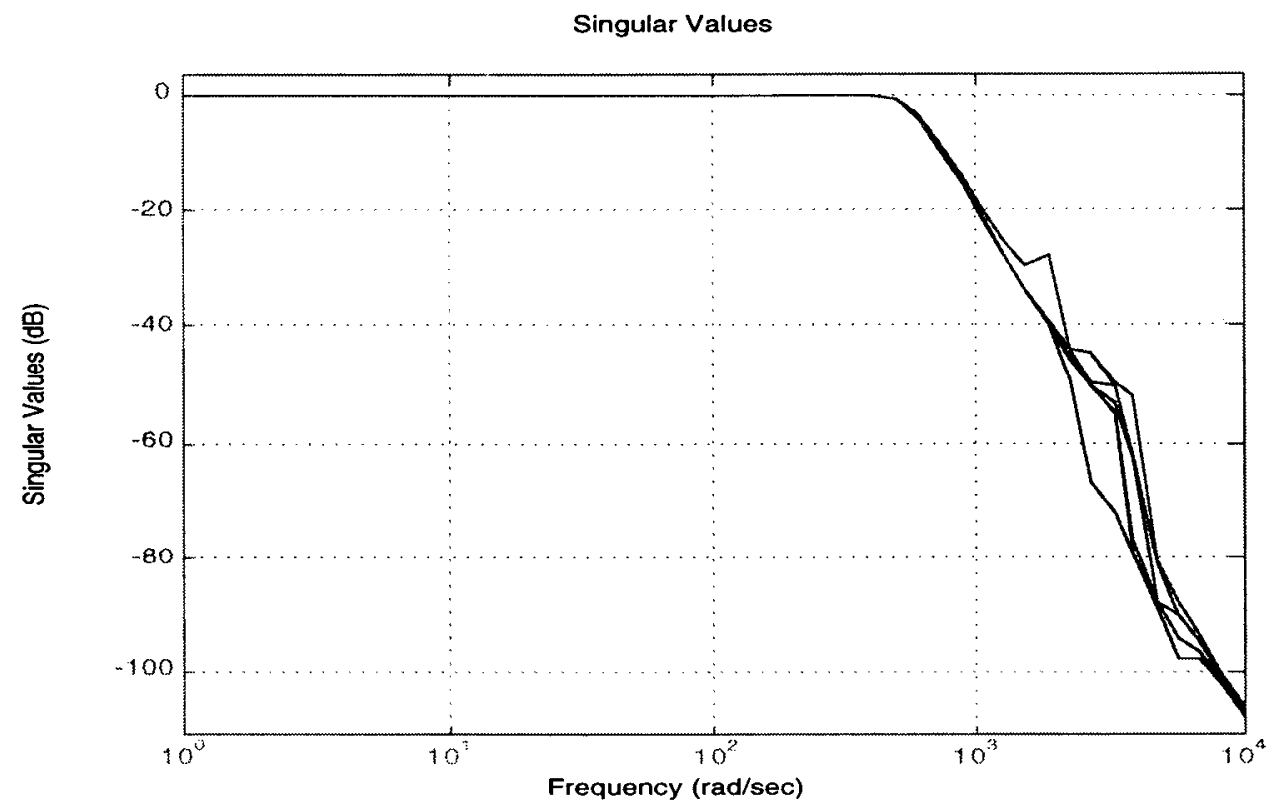

Figure 6.29 Frequency plot of complimentary sensitivity $\left(\mathrm{T}_{\mathrm{y}}\right)$ using $\mathrm{H}_{\infty}$ controller 


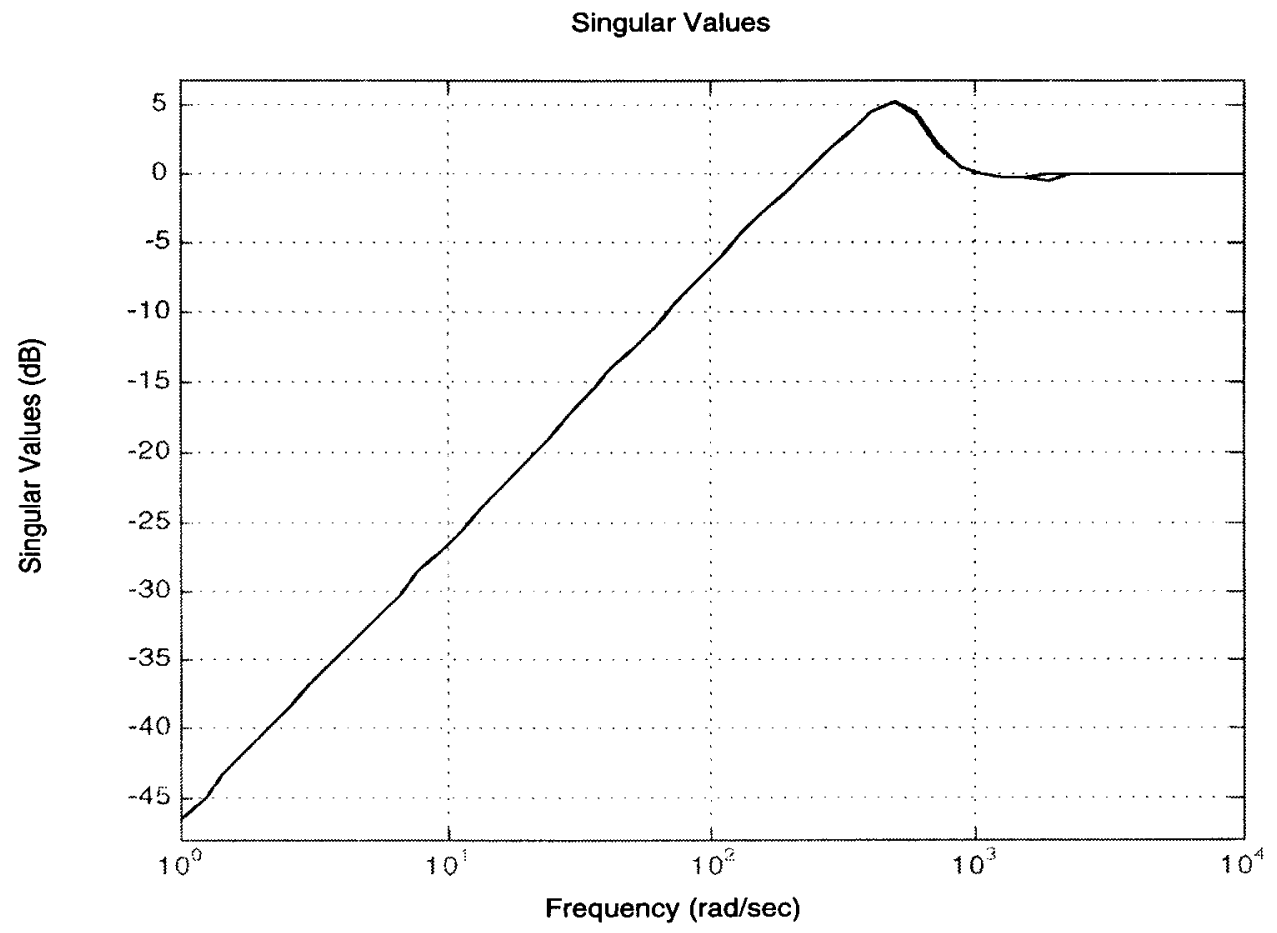

Figure 6.30 Frequency plot of sensitivity $\left(\mathrm{S}_{\mathrm{y}}\right)$ using $\mathrm{H}_{\infty}$ controller 


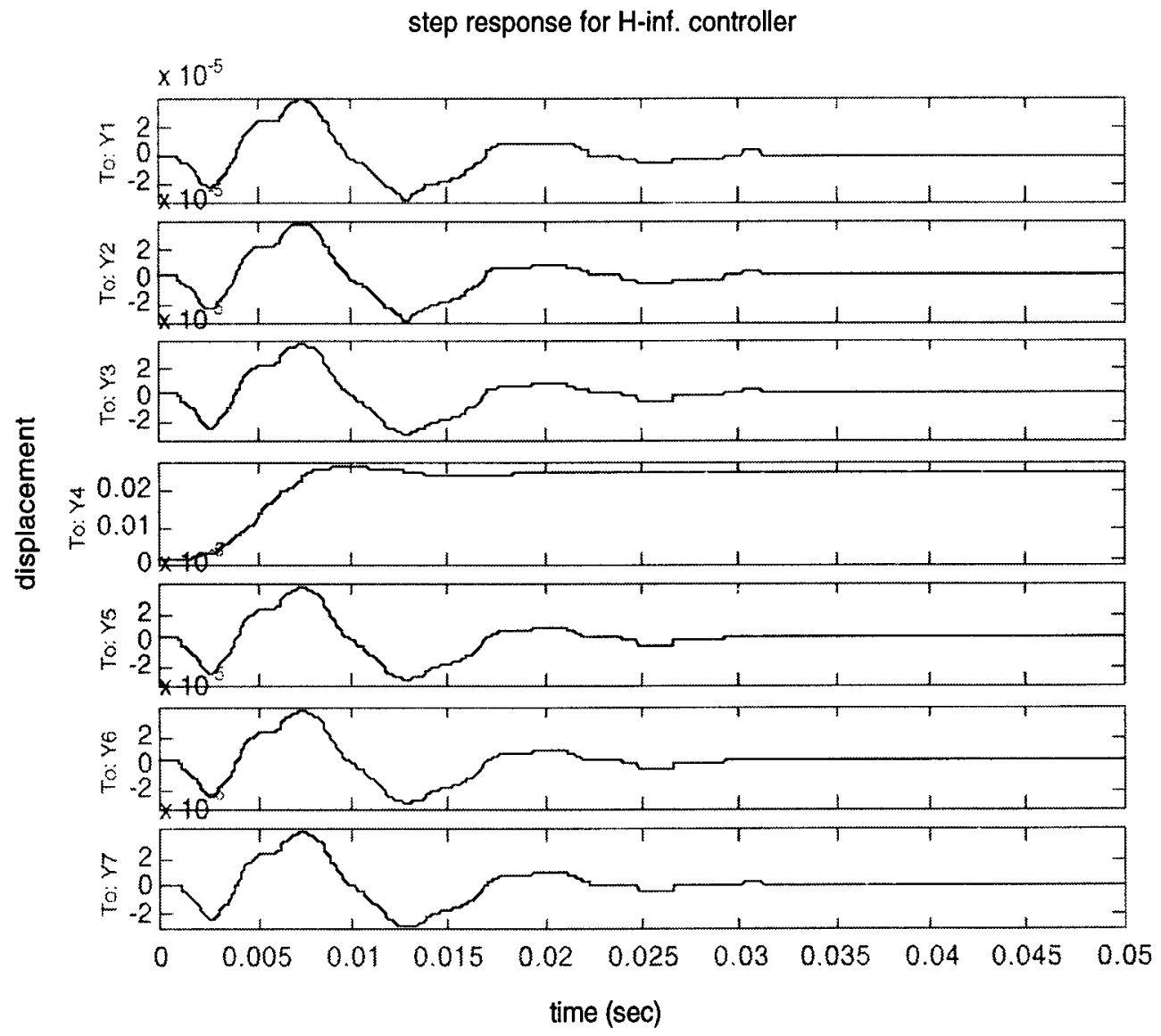

Figure 6.31 Step response using $\mathrm{H}_{\infty}$ controller

\subsection{Conclusion}

In this chapter, a multi-input multi-output (MIMO) $\mathrm{H}_{\infty}$ controller for dynamic surface shape control was developed to improve closed loop performance in the presence of disturbances and uncertainties. Initially, general design objectives were overviewed with emphasis placed on stability, performance, and robustness. Next, a SISO controller was developed based on a proportional-integral approach, which gave some general insight to the characteristics of the dynamic system. Then, MIMO controllers were developed based on a traditional proportional-integral (PI) method and the more modern $\mathrm{H}_{\infty}$ method. Both 
MIMO controllers were developed utilizing the dynamic model developed in Chapter 5. Lastly, comparisons were made between the proportional-integral (PI) and $\mathrm{H}_{\infty}$ controller designs, which clearly showed the $\mathrm{H}_{\infty}$ controller having superior performance and robustness characteristics. 


\section{CHAPTER 7 - CONCLUSIONS AND RECOMMENDATIONS}

\subsection{Conclusions}

The purpose of this thesis was the development of static and dynamic models for control purposes and the development of a multi-input multi-output (MIMO) controller for precision surface shape control applications. Building prototypes to complete design optimizations or controller development can be costly or impractical. This shortfall, puts significant value in developing accurate modeling and control simulation approaches. Such development has the potential for use in a wide variety of applications, such as adaptive optics, aircraft flutter control and noise control for marine vessels. The development involved a detailed literature search of each area, development and validation of a static model, MIMO control application of the static model, development and validation of a MIMO dynamic model, and lastly the development of a MIMO controller using a modern control approach.

To begin the development, in Chapter 1, a general description of the problem was presented, outlining the need for a more focused approach on modeling and control simulation in the field of adaptive optics. Additionally, a literature search was performed to investigate existing techniques and applications applied in various fields, which showed that these modeling approaches had not been previously applied to adaptive optics.

Next, in Chapter 2, a general overview of adaptive optics systems was presented. This included areas such as the deformable mirror, controller and sensor configuration. A specific example was presented, which utilized the National Ignition Facilities (NIF) adaptive optic system. The deformable mirror configurations covered both continuous and segmented mirror approaches which utilized piezo-electric actuators. Both direct and indirect sensing approaches were discussed, with the Shack-Hartmann sensor discussed in detail. 
With the general overview of adaptive optics complete, in Chapter 3, the static model was developed. The model was developed using the Finite Element Method through the commercially available I-DEAS software. With the modeling complete, experimental data was collected on the NIF prototype deformable mirror utilizing a large aperture interferometer system at Lawrence Livermore National Laboratory. Lastly, experimental results were compared to finite element results using a normalization approach and a statement of model fidelity was established. Model and experimental peak-to-valley differences were a maximum of $+/-6$ percent, which is comparable to material property uncertainties.

Utilizing the validated static model, in Chapter 4, a static control approach was implemented using existing control approaches. The control model was developed based on a linear least squares approach and the sensor was based on the Shack-Hartmann wavefront sensor. Using this control model, typical aberrations were corrected to show the general performance. Furthermore, this control model shows significant value in simulating static surface shape control problems with the ability to test various deformable mirror, sensor, and control algorithm designs. Lastly, the control model was utilized to compare the residual errors of a typical Gaussian plant model versus the validated finite element model. As expected, the Gaussian model produced errors 2 to 29 times greater than the finite element model for various aberrations.

In Chapter 5, new dynamic models were developed of the NIF prototype deformable mirror in order to facilitate the design of SISO and MIMO control approaches. Based on an extensive literature review, a modal density model reduction approach was employed. This approach uses a priori information to reduce model state size in the model development stage. The dynamic equations of motion were developed utilizing bond graphs. With the equations formulated, they were then coded using the Matlab programming language. Both time domain and frequency domain open loop responses were simulated. Next, experimental frequency response data was collected on the NIF 
prototype deformable mirror. Lastly, this experimental data, and existing literature data was used to validate the dynamic model.

Chapter 6 focused on the development of SISO and MIMO controllers for surface shape control. First, a SISO proportional-integral controller was developed to determine general characteristics of the dynamic system. Then, a new MIMO controller was developed utilizing a proportional-integral controller to establish a system performance baseline. Next, a new MIMO controller was developed based on the $H_{\infty}$ method. Simulations were completed for both controllers and the $\mathrm{H}_{\infty}$ design showed superior characteristics as expected, in terms of robustness and disturbance rejection. The $\mathrm{H}_{\infty}$ design improved rise time by approximately a factor of two over the PI design and had better decoupling characteristics, which relates directly to improved surface shape control performance. Lastly, when evaluating robustness characteristics, the $\mathrm{H}_{\infty}$ design maintained stability when the PI design became unstable.

Lastly, a few comments will be made concerning how these modeling and controller developments can be used to design the next generation of deformable mirror and/or surface shape control system. Utilizing the finite element modeling approach, a static model can be developed and the output used as the plant model for the control model. Depending on the system, the control model may utilize a sensor or use the surface displacement points for the least squares fitting algorithm. With some knowledge of the expected disturbances, the control model can be used to evaluate various plant models (i.e., using the finite element model), sensor configurations, and control algorithms. With a baseline static model established, a dynamic model can be developed using the modeling approach presented in this thesis, so that controller characteristics can be studied. First, start with the SISO model to provide general insight, then expand to the more complex MIMO model. With no experimental data, model parameters need to be estimated. With 
the dynamic model complete, various control algorithms can be evaluated for stability, performance, and robustness. Utilizing these accurate modeling and control simulation approaches will add significant value during the design stage in the absence of building costly prototypes to complete design optimizations or controller development.

\subsection{Recommendations}

As a final section of this thesis, recommendations concerning future work on surface shape modeling and control will be made. Although this thesis focused on model development and control, many tangent issues arose.

Concerning the static surface shape control approach, additional research could be completed which investigates the model's parameter space of a given design to reduce the root-mean-square fitting error for a given aberration. This parameter space should include, actuator density, actuator spacing, actuator pad size and actuator configurations. Although research has been completed in this field, a more complete study would be invaluable.

Concerning the dynamic surface shape model, additional areas-could benefit from additional research. The first is to model the non-linear characteristics of piezo-electric actuators. Although the non-linearities are small, their uncertainty may cause problems if neglected. Secondly, it is recommended to add the higher frequency characteristics of the system, such as plate modes, and study the observability and controllability of these modes. The challenge will be to implement these additional modes and keep the system size reasonably low. Lastly, additional dynamic tests for quantitative model validation would be valuable.

Another area which would be interesting to research, is to combine the finite element results with the state space model. This would allow a full surface dynamic description of the mirror and still allow for control system development based on state space methods. 
Lastly, the controller related research can be expanded. Controller reduction techniques will need to be implemented and/or developed for the $\mathrm{H}_{\infty}$ controller performance benefits to be realized for large scale systems. However, as hardware and software capabilities improve, this shortfall will be minimized. Secondly, research focusing on actuator saturation issues utilizing the $\mathrm{H}_{\infty}$ methodology could be studied. Lastly, development of control expansion techniques, such as expanding the seven actuator controller to a n-actuator controller without developing a larger model would be invaluable. 


\section{REFERENCES}

Aoki, M., 1978, "Some Approximate Methods for Estimation and Control of Large Scale Systems," IEEE Transactions of Automatic Control, Vol. 23/2, pp. 173-182

Apollonov, V. V., Vdovin, G. V., Ivanova, E. A., Prokhorov, A. M., Shetkin, S. A., 1990, "Analytic Model of an Adaptive Mirror in the Form of a Thin Plate with Discrete Actuators," Soviet Journal of Quantum Electronics, Vol. 20, No. 11, pp. 1414-1418.

Astrom, K. J., 1983, "Theory and Applications of Adaptive Control-A Survey," Automatica, Vol. 19, No. 5, pp. 471-486.

Bacon, B.J., 1989, “Multivariable Frequency-Weighted Order Reduction,” J. Guidance, Vol. 12, No. 1, Jan.-Feb.

Balas, G. J., Doyle, J. C., 1990, "Robustness on Performance Tradeoffs in Control Design for Flexible Structures," Proc. of 29th Conference on Decision and Control, pp. 2999-3013.

Banyasz, C. S., Keviczky, L., 1993, "Design of Adaptive PID Regulators Based on Recursive Estimation of the Process Parameters," Journal of Process Control, Vol. 3, No. 1, pp. 53-59.

Bigelow, B.C., Bingham, R.G., Walker, D.D., 1994, “Optimizing a Deformable Secondary Mirror for Adaptive Optics," Adaptive Optics in Astronomy, SPIE Vol. 2201, pp.800-810.

Blanton, R. L., Sadek, I. S., 1992, "Optimal Active Pointwise Control of Vibrating Thin Plates," Journal of the Franklin Institute, Vol. 329, No. 5, pp. 801-815.

Boyer, C., Griffard, J.P., 1991, "Adaptive Optics, Transfer Loop Modeling," SPIE Vol. 1542 Active and Adaptive Optical Systems, pp. 46-61.

Brewer, J.W., 1991, Linear Feedback Control Theory - EME272A Class Notes, University of California, Davis.

Brewer, J.W., 1995, Linear, Multivariable, Feedback Control Theory - EME272B Class Notes, University of California, Davis.

Bushnell, D., 1979, "Control of Surface Configuration by Application of Concentrated Loads," AIAA Journal, Vol. 7, No. 1, pp. 71-77.

Chiarappa, D. J., Claysmith, C. R., 1981, "Deformable Mirror Surface Control Techniques," Journal of Guidance and Control, Vol. 4, No. 1, pp. 7-34.

Cielinski, A.J., 1991."Wavefront Control Model of A Beam Control Experiment," SPIE Vol. 1542 Active and Adaptive Optical Systems, pp. 434-448.

Creedon, J. F., Lindgren, A. G., 1970, "Control of the Optical Surface of a Thin, Deformable Primary Mirror with Application to an Orbiting Astronomical Observatory," Automatica, Vol. 6, pp. 643-660. 
Dimitriadis, E. K., Fuller, C.R., 1989, "Investigation of Active Control of Sound Transmission Through Elastic Plates Using Piezoelectric Actuators." AIAA 27th Aerospace Sciences Conference, Reno, Nevada, Jan. 8-12, Paper 89-106.

Doyle, J.C., Glover, K., Khargonekar, P.P., Francis, B.A., 1989, "State-Space Solutions to Standard $\mathrm{H}_{2}$ and $\mathrm{H}_{\infty}$ Control Problems," IEEE Transactions on Automatic Control, Vol. 34, No. 8, pp.831-846.

Ealey, M.A., Wellman J.A., 1991, "Deformable Mirrors: Design Fundamentals, Key Performance Specifications and Parametric Trades," Active and Adaptive Optical Components, SPIE Vol. 1543, pp. 36-51.

Ealey, M.A., 1991, "Active and Adaptive Optical Components: The Technology and Future Trends," Active and Adaptive Optical Components, SPIE Vol. 1543, pp. 2-34.

Ealey, M. A., Wellman, J. A., 1994, "Xinetics Low Cost Deformable Mirrors with Actuator Replacement Cartridges," Adaptive Optics in Astronomy, SPIE Vol. 2201, pp. 680-687.

Ellerbroek, B. L., 1994, "Optimizing Closed Loop Adaptive Optics Performance Using Multiple Control Bandwidths," Adaptive Optics in Astronomy, SPIE Vol. 2201, pp. 935948.

Fanson, J., Anderson, E., Rapp, D., 1990, "Active Structures for Control of Large Precision Optical Systems," Optical Engineering, Vol. 29, No. 11, pp. 1320-1327.

Fortuna, L., Nunnari, G., Gallo, A., 1992, Model Order Reduction Techniques with Applications in Electrical Engineering, Springer-Verlag York Berlin Heidelberg.

Freeman, R. H., Garcia, H. R., 1982, "High-speed Deformable Mirror System," Applied Optics, Vol. 21, No. 4, February 15, pp. 589-595.

Furber, M.E., Blaszak, D.D., Pieri, M.R., 1994a, “ Correctability Modeling of a Large Deformable Mirror," Adaptive Optics in Astronomy, SPIE Vol. 2201, pp. 856-866.

Furber, M.E., Cox, C.D., Blaszak, D.D., Houghton C.D., 1994b, "Interferometric Measurement and Analysis of Influence Functions for a Large Deformable Mirror," Adaptive Optics in Astronomy, SPIE Vol. 2201, pp. 788-799.

Gregory, C.Z., 1984, "Reduction of Large Flexible Spacecraft Models using Internal Balancing Theory," J. Guidance, Vol. 7, No. 6, Nov.-Dec.

Giurgiutiu, V., Chaudhry, Z., Rogers, C.A., 1995, "Energy-Based Comparison of SolidState Actuators," Virginia Tech, Center for Intelligent Material Systems and Structures, Report No. CIMSS 95-101.

Gully, S. W., Huang, J., Denis, N., Looze, D. P., Wirth, A., Jankevics, A. J., Castanon, D. A., 1994, "Experiments with Adaptive Nonlinear Control Systems for Atmospheric Correction," Adaptive Optics in Astronomy, SPIE Vol. 2201, pp. 920-934.

Hiddleston, H.R., Lyman, D.D., Schafer, E.L., 1991, "Comparisons of Deformable Mirror Models and Influence Functions," SPIE Vol. 1542 Active and Adaptive Optical Systems, pp. 20-33. 
Hyde, J., Seering, W., 1991, "Using Input Command Preshaping to Suppress Multiple Mode Vibration," IEEE Conference on Robotics \& Automation, Sacramento, pp. 26042609.

Junkins, J.L., 1990, Mechanics and Control of Large Flexible Structures, American Institute of Aeronautics and Astronautics, Inc.

Karnopp, D.C., Margolis, D.L., Rosenberg, R.C.,1990, System Dynamics: A Unified Approach, John Wiley \& Sons, Inc.

Karnopp, D.C., 1996, personal communication on using a C-field to model an electrostrictive actuator.

Kibblewhite, E., Smutko, M. F., and Shi F., 1994, "The Effect of Hysteresis on the Performance of Deformable Mirrors and Methods of its Compensation," Adaptive Optics in Astronomy, Vol. 2201, pp. 754-761.

Kokotovic, P.V., Sannuti, P., 1986,"Singular Perturbation Method for Reducing the Model Order in Optimal Control Design," IEEE Transactions on Automatic Control, Vol. $13 / 4$.

Koch, J., 1998, personal communication on testing results for the large aperture interferometer system.

Kreyszig, E., 1988, Advanced Engineering Mathematics, John Wiley \& Sons, Inc.

La Fiandra, C., Goldstein, G.A., Mehta, P.K., Zacharias, R.A., Winters, S.E., 1998, "National Ignition Facility Deformable Mirror," Solid State Lazers. for-Applications to Inertial Confinement Fusion, Montery CA. June.

Lawry, M.H., 1997, SDRC Master Series Student Guide, SDRC..

Lillard, R. L., Schell, J. D., 1994, "High-Performance Deformable Mirror for Wavefront Compensation," Adaptive Optics in Astronomy, SPIE Vol. 2201, pp. 740-753.

Livne, E., Li, Wei-Lin, 1995, "Aeroservoelastic aspects of Wing/Control Surface Platform Shape Optimization," Vol. 55, No. 2, pp. 302-311.

Luo, N., Delasen, M., 1993, "State Feedback Sliding Mode Control of a Class of Uncertain Time Delay Systems," IEEE Proceedings-D control Theory and Applications, Vol. 140, No. 4, pp. 261-274.

Lyakhov, D. M., 1994, "Optimizing the Control of the Shape of the Optical Surface of Plates with Point Connections," Journal of Optical Technology, Vol. 61, No. 7, pp. 518522.

Lyakhov, D. M., Shanin, O. I., 1994, "Optimizing the Control of the Shape of the Optical Surface of Circular Plates," Journal of Optical Technology, Vol. 61, No. 7, pp. 522-525.

Margolis, D. L., 1976, "Finite Mode Bond Graph Representation of Vehicle-Guideway Interaction Problems," Journal of the Franklin Institute, Vol. 302, No. 2, pp. 1-17. 
Margolis, D. L., 1980, "Dynamical Models for Multidimensional Structures Using Bond Graphs," Journal of Dynamic Systems, Measurements and Controls, Vol. 102, pp. 180187.

Margolis, D. L., 1985, "A Survey of Bond Graph Modeling for Interacting Lumped and Distributed Systems," Journal of the Franklin Institute, Vol. 319, No. 1/2, pp. 125-135.

Marlow, W.C., 1994, "Dynamics of Deformable Mirror Actuators," Optical Engineering, Vol. 33, No. 4, pp. 1016-1026.

Mayne, D.Q., 1996, Multivariable Control System Design - EEC252 Class Notes, University of California, Davis.

Meirovitch, L., 1967, Analytical Methods in Vibrations, The MacMillan Company.

Meirovitch, L., 1990, Dynamics and Control of Structures, John Wiley \& Sons, Inc.

Nicholson, J.W., Bergman, L.A., 1986, "Free Vibration of Combined Dynamical Systems," Journal of Engineering Mechanics, Vol. 112, No. 1, pp. 1-13.

Patterson, S.R., 1994, Treatment of Uncertainty and Errors - Notes, ASPE conference tutorial.

Peter, K., Isermann R., 1993, "Parameter Adaptive PID Control Based on Continuous Time Process Models," Control Theory and Advanced Technology, Vol. 9, No. 1, pp. 2738.

Rad, A. B., Lo, L. W., 1995, "Adaptive PID Control of Systems with Unknown Delay," International Journal of Systems Science, Vol. 26, No. 3, ppr 519-638.

Rattan, K. D., 1989, "Compensating for Computational Delay in Digital Equivalent of Continuous Control Systems," IEEE Transactions on Automatic Control, Vol. 34, No. 8, pp. 895-899.

Russell, R., 1996, "Don't Trust the Pretty Pictures," Machine Design CAM/CAD Industry Report, May.

Sacks, R.A., 1998a, personal communication on converting Phase Shifts' data and normalization method.

Sacks, R.A., 1998b, personal communication on shifting Gaussian influence functions.

Safonov, M.G., Limebeer, D.J.N., Chiang, R.Y., 1989, "Simplifying the Ho Theory via Loop-shifting, Matrix-pencil and Descriptor concepts," International Journal of Control, Vol. 50, No. 6, pp. 2467-2488.

Safonov, M.G., Chiang, R.Y.,1992, Robust Control Toolbox User's Guide, The MathWorks, Inc.

Salmon, J.T., Bergum, J.W., Kartz, M.W., Presta, R.W., Swift, C.D., 1993, "Wavefront Correction System Based on an Equilateral Triangular Arrangement of Actuators," SPIE, Vol. 1920, pp. 20-28. 
Schaechter, D. B., 1981, "Optimal Local Control of Flexible Structures," Journal of Guidance and Control, Vol. 4, No. 1, Jan-Feb, pp. 22-26.

Shishakov, K. V., Shmal'gauzen, V. I., 1992, "Optimization of Surface Shape Control in Feedback Systems," Automation and Remote Control, No. 53, pp. 1866-1871.

Skelton, R.E., 1980, "Cost Decomposition of Linear Systems with Application to Model Reduction," International Journal of Control, Vol. 32/6, pp. 1031-1055.

Skelton, R.Z., Hughes, P.C., Hablani, H.B., 1982, "Order Reduction for Models of Space Structures Using Modal Cost Analysis," J. Guidance and Control, Vol. 5, No. 4, July-Aug.

Slotine, J.-J.E., Li, W., 1991, Applied Nonlinear Control, Prentice Hall.

Sueur, C., Dauphintanguy, G., 1991, "Bond-Graph Approach for Structural Analysis of Mimo Linear Systems," Journal of the Franklin Institute, Vol. 328, No. 1, pp. 55-70.

Tyson, R. K., 1998, Principles of Adaptive Optics, Academic Press, Inc.

Vincent, T. L., Lin, Y. C., Joshi, S. P., 1990, "Controlling a Flexible Plate to Mimic a Rigid One," Control and Dynamic Systems, Vol. 35, pp. 87-135.

Walker, L. A., Yaneskek, P. P., 1976a, "The Damping of the Plate Vibrations by Means of Multiple Active Control Systems," Journal of Sound and Vibration, Vol. 46, pp. 177193.

Walker, L. A., Yaneskek P. P., 1976b, "Characteristics of an Active Feedback System for the Control of Plate Vibrations," Journal of Sound and Vibration, Vol. 46, pp. 157-176.

Wilson, D.A., Mishra, R.N., 1979, "Optimal reduction of Multivariable Systems," International Journal of Control, Vol. 29/2, pp. 267-278

Winters, S.E., Arnold, T.A., Bliss, E.S., Bergum, J.W., Kartz, M.A., Salmon, J.T., Stolz, C.J., Van Wonterghen, B.M., Wegner, P.J., Weinzapfel, C.L, Whistler, W.T, Zacharias, R.A., 1998, "Design Overview of a Large Aperture Deformable Mirror for the National Ignition Facility,” ASPE Annual Meeting, St. Louis, Missouri. October.

Wirth, A., Jankevics, A. J., 1993, "Adaptive Nonlinear Control Systems for Atmospheric Correction," SPIE, Adaptive Optics in Astronomy, Vol. 1920, pp. 245-262.

Zacharias, R., Bliss, E., Feldman, M., Grey, A., Henesian, M., Kock, J., Lawson, J., Sacks, R., Salmon, T., Toeppen, J., Van Atta, L., Winters, S., 1998, "The National Ignition Facility Wavefront Control System," Solid State Lasers for Application to Inertial Confinement Fusion, Montery CA. June. 
APPENDIX A - STATIC MODEL CONTROL CODE 
$\%$ m-file for static control model.

$\%$ this program reads in 39 influence function files (1024 x 1), reads in or calculates an $\%$ aberration file, uses a Hartmann sensor model, and lastly a least squares control $\%$ algorithm.

$\%$ Program written by : Scott E. Winters

$\%$ Date: $7 / 98$

$\%$ reading in 39 influence function files

clear

$\mathrm{n}=.1$;

pause;

nif01REF4

pause(n)

nif02REF4

pause(n)

nif03REF4

pause(n)

nif04REF4

pause(n)

nif05REF4

pause(n)

nif06REF4

pause(n)

nif07REF4

pause(n)

nif08REF4

pause(n)

nif09REF4

pause(n)

nif10REF4

pause(n)

nif11REF4

pause(n)

nif12REF4

pause(n)

nif13REF4

pause(n)

nif14REF4

pause(n)

nif15REF4

pause(n)

nif16REF4

pause(n)

nif17REF4

pause(n)

nif18REF4

pause(n)

nif19REF4

pause(n)

nif20REF4

pause(n)

nif21REF4

pause(n)

nif22REF4 


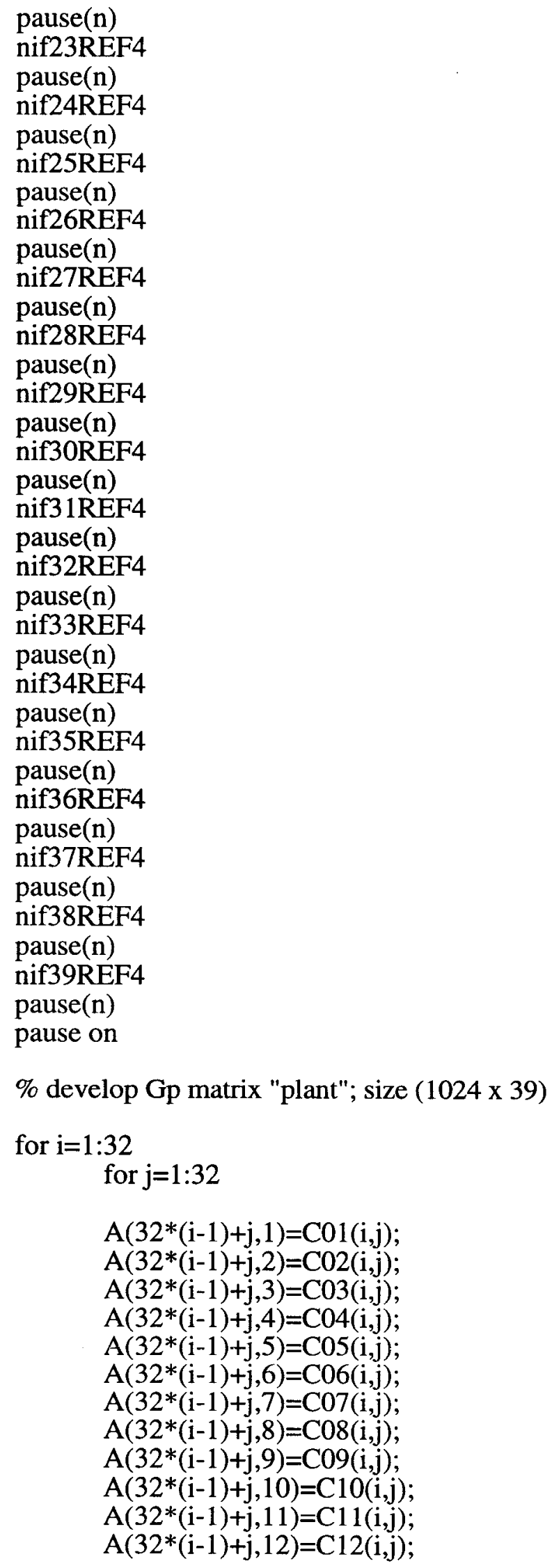




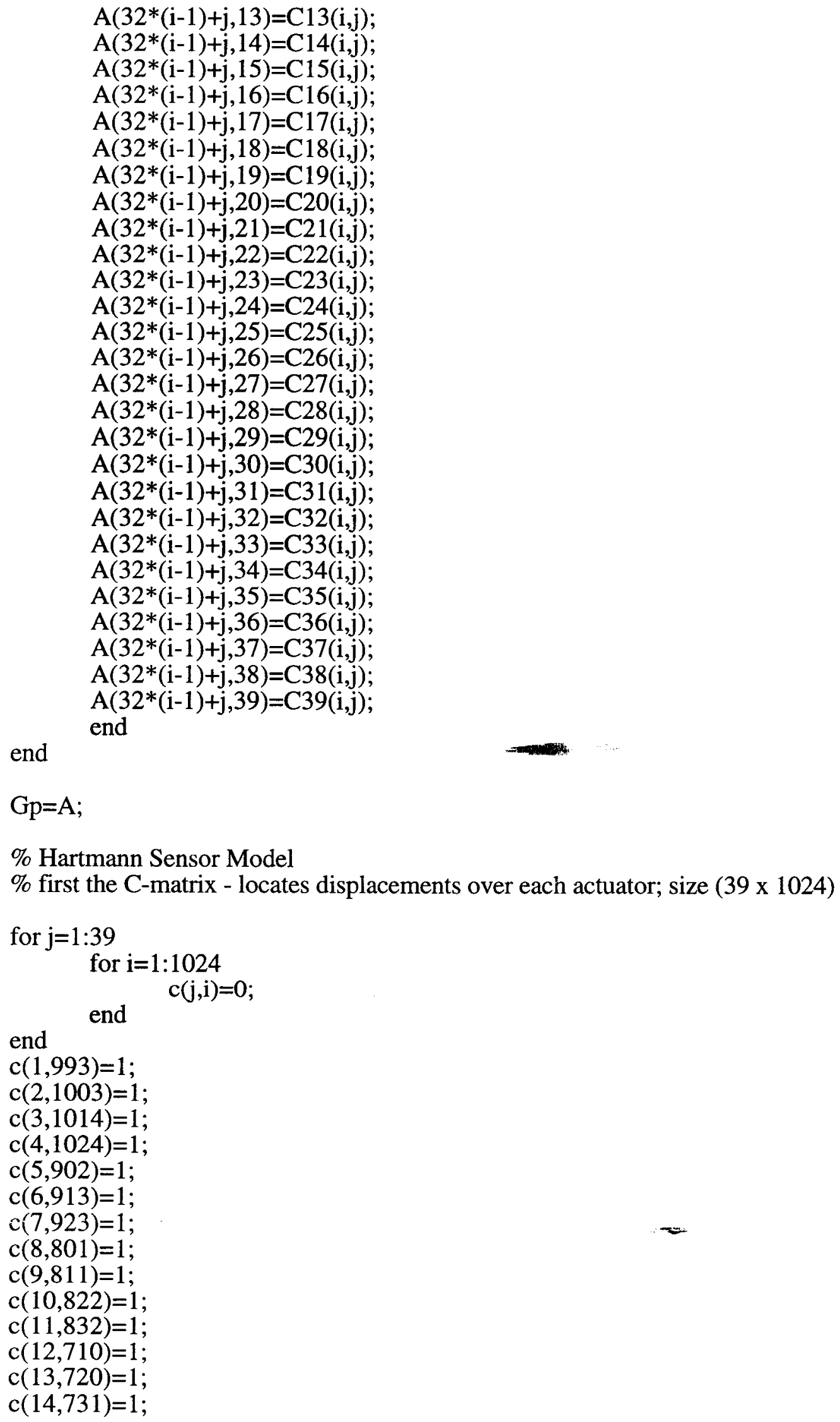




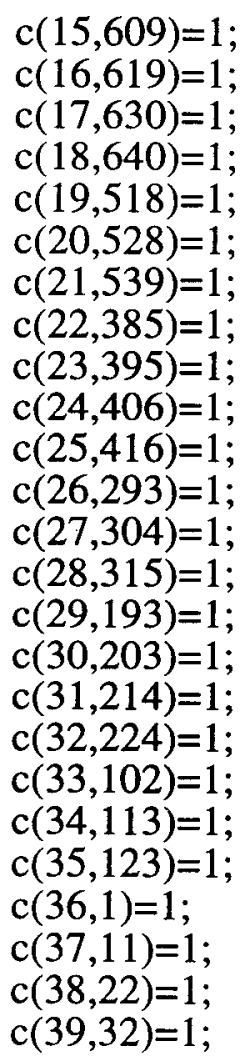

$\%$ B-matrix development - finite difference matrix; size ( 154 x 39)

$$
\begin{aligned}
& \text { for } j=1: 154 \\
& \text { for } i=1: 39 \\
& \text { end } b(j, i)=0
\end{aligned}
$$

end

\%type I sensor

$\mathrm{a}=[1 ; 4 ; 7 ; 11 ; 14 ; 17 ; 18 ; 21 ; 24 ; 28 ; 31 ; 34 ; 35 ; 38 ; 41 ; 45 ; 48 ; 51 ; 52 ; 55 ; 58 ; 62 ; 65 ; 68 ; 69 ; 72 ; 75]$; $\mathrm{e}=[1 ; 2 ; 3 ; 5 ; 6 ; 7 ; 8 ; 9 ; 10 ; 12 ; 13 ; 14 ; 15 ; 16 ; 17 ; 19 ; 20 ; 21 ; 22 ; 23 ; 24 ; 26 ; 27 ; 28 ; 29 ; 30 ; 31]$; $\mathrm{f}=[3 ; 6 ; 9 ; 10 ; 13 ; 16 ; 20 ; 23 ; 26 ; 27 ; 30 ; 33 ; 37 ; 40 ; 43 ; 44 ; 47 ; 50 ; 54 ; 57 ; 60 ; 61 ; 64 ; 67 ; 71 ; 74 ; 77]$; $\mathrm{g}=[2 ; 3 ; 4 ; 5 ; 6 ; 7 ; 9 ; 10 ; 11 ; 12 ; 13 ; 14 ; 16 ; 17 ; 18 ; 19 ; 20 ; 21 ; 23 ; 24 ; 25 ; 26 ; 27 ; 28 ; 30 ; 31 ; 32]$;

for $\mathrm{i}=1: 27$

$\% \mathrm{x}$-direction $\mathrm{b}(\mathrm{a}(\mathrm{i}), \mathrm{e}(\mathrm{i})+4)=0.0144$ $\mathrm{b}(\mathrm{a}(\mathrm{i}), \mathrm{e}(\mathrm{i}))=-0.0072$;

$\mathrm{b}(\mathrm{a}(\mathrm{i}), \mathrm{e}(\mathrm{i})+7)=-0.0072$;

$\mathrm{b}(\mathrm{f}(\mathrm{i}), \mathrm{g}(\mathrm{i})+3)=-0.0144$;

$\mathrm{b}(\mathrm{f}(\mathrm{i}), \mathrm{g}(\mathrm{i}))=0.0072$;

$\%$-direction $b(f(i), g(i)+7)=0.0072$;

$\mathrm{b}(\mathrm{a}(\mathrm{i})+77, \mathrm{e}(\mathrm{i}))=0.0124$;

$\mathrm{b}(\mathrm{a}(\mathrm{i})+77, \mathrm{e}(\mathrm{i})+7)=-0.0124$;

$\mathrm{b}(\mathrm{f}(\mathrm{i})+77, \mathrm{~g}(\mathrm{i}))=0.0124$;

$\mathrm{b}(\mathrm{f}(\mathrm{i})+77, \mathrm{~g}(\mathrm{i})+7)=-0.0124$; 
end

\%type II sensor

$\mathrm{a}=[12 ; 15 ; 19 ; 22 ; 25 ; 29 ; 32 ; 36 ; 39 ; 42 ; 46 ; 49 ; 53 ; 56 ; 59 ; 63 ; 66]$;

$\mathrm{e}=[5 ; 6 ; 8 ; 9 ; 10 ; 12 ; 13 ; 15 ; 16 ; 17 ; 19 ; 20 ; 22 ; 23 ; 24 ; 26 ; 27]$;

for $\mathrm{i}=1: 17$

$\% \mathrm{x}$-direction

$\mathrm{b}(\mathrm{a}(\mathrm{i}), \mathrm{e}(\mathrm{i}))=-0.0144$;

$\mathrm{b}(\mathrm{a}(\mathrm{i}), \mathrm{e}(\mathrm{i})+7)=-0.0144$;

$\mathrm{b}(\mathrm{a}(\mathrm{i}), \mathrm{e}(\mathrm{i})+1)=0.0144$;

$\%$-direction $\mathrm{b}(\mathrm{a}(\mathrm{i}), \mathrm{e}(\mathrm{i})+8)=0.0144$;

$$
\begin{aligned}
& \text { b(a(i)+77,e(i))=0.00207; } \\
& \text { b(a(i)+77,e(i)+7)=-0.00207; } \\
& \text { b(a(i)+77,e(i)+1)=0.00207; } \\
& \text { b(a(i)+77,e(i)+8)=-0.00207; } \\
& \text { b(a(i)+77,e(i)-3)=0.00415; } \\
& \text { b(a(i)+77,e(i)+11)=-0.00415; }
\end{aligned}
$$

end

$\%$ specific cases

$\%$ lenslets \#2

$$
\begin{aligned}
& b(2,1)=-0.0144 \\
& b(2,1+7)=-0.0144 \\
& b(2,1+1)=0.0144 \\
& b(2,1+8)=0.0144 \\
& b(2+77,1)=0.00207 \\
& b(2+77,1+7)=-0.00207 \\
& b(2+77,1+1)=0.00207 \\
& b(2+77,1+8)=-0.00207 \\
& b(2+77,1+11)=-0.00415
\end{aligned}
$$

$\%$ lenslets \#5

$$
\begin{aligned}
& b(5,2)=-0.0144 \\
& b(5,2+7)=-0.0144 \\
& b(5,2+1)=0.0144 \\
& b(5,2+8)=0.0144 \\
& b(5+77,2)=0.00207 \\
& b(5+77,2+7)=-0.00207 \\
& b(5+77,2+1)=0.00207 \\
& b(5+77,2+8)=-0.00207 \\
& b(5+77,2+11)=-0.00415
\end{aligned}
$$

$\%$ lenslets \#8

$$
\begin{aligned}
& b(8,3)=-0.0144 \\
& b(8,3+7)=-0.0144 \\
& b(8,3+1)=0.0144 \\
& b(8,3+8)=0.0144
\end{aligned}
$$




$$
\begin{aligned}
& b(8+77,3)=0.00207 \\
& b(8+77,3+7)=-0.00207 \\
& b(8+77,3+1)=0.00207 \\
& b(8+77,3+8)=-0.00207 \\
& b(8+77,3+11)=-0.00415
\end{aligned}
$$

$\%$ lenslets \#70

$$
\begin{aligned}
& b(70,29)=-0.0144 ; \\
& b(70,29+7)=-0.0144 ; \\
& b(70,29+1)=0.0144 ; \\
& b(70,29+8)=0.0144 ; \\
& b(70+77,29)=0.00207 \\
& b(70+77,29+7)=-0.00207 \\
& b(70+77,29+1)=0.00207 ; \\
& b(70+77,29+8)=-0.00207 \\
& b(70+77,29-3)=0.00415
\end{aligned}
$$

$\%$ lenslets \#73

$$
\begin{aligned}
& \text { b }(73,30)=-0.0144 ; \\
& \text { b(73,30+7)=-0.0144; } \\
& \text { b }(73,30+1)=0.0144 ; \\
& b(73,30+8)=0.0144 ; \\
& \text { b }(73+77,30)=0.00207 \\
& b(73+77,30+7)=-0.00207 \\
& \text { b }(73+77,30+1)=0.00207 \\
& b(73+77,30+8)=-0.00207 \\
& b(73+77,30-3)=0.00415
\end{aligned}
$$

\% lenslets \#76

$$
\begin{aligned}
& \text { b(76,31)=-0.0144; } \\
& \text { b(76,31+7)=-0.0144; } \\
& \text { b(76,31+1)=0.0144; } \\
& \text { b(76,31+8)=0.0144; } \\
& \text { b(76+77,31)=0.00207; } \\
& \text { b }(76+77,31+7)=-0.00207 \\
& \text { b }(76+77,31+1)=0.00207 ; \\
& b(76+77,31+8)=-0.00207 \\
& \text { b }(76+77,31-3)=0.00415
\end{aligned}
$$

\% generate sensor matrix, Gs; size (154 x 1024)

\section{$\mathrm{Gs}=\mathrm{b}^{*} \mathrm{c}$;}

$\%$ set up displacements from function trying to fit "aberration" or read aberration file $\%$ load aberration \% file must be saved as aberration.m ; size (1024 x 1) for $i=1: 32$

$$
\begin{aligned}
& \text { for } \mathrm{j}=1: 32 \\
& \% \mathrm{~F}(32 *(\mathrm{i}-1)+\mathrm{j})=0.001 ; \quad \% \text { flat input } \\
& \% \mathrm{~F}(32 *(\mathrm{i}-1)+\mathrm{j})=0.000000105^{*} \mathrm{x}(\mathrm{j})^{\wedge} 2 ; \quad \% \text { 2nd order } \\
& \% \mathrm{~F}(32 *(\mathrm{i}-1)+\mathrm{j})=0.00000000000065625^{*} \mathrm{x}(\mathrm{j})^{\wedge} 4 ; \quad \% \text { 4th order }
\end{aligned}
$$




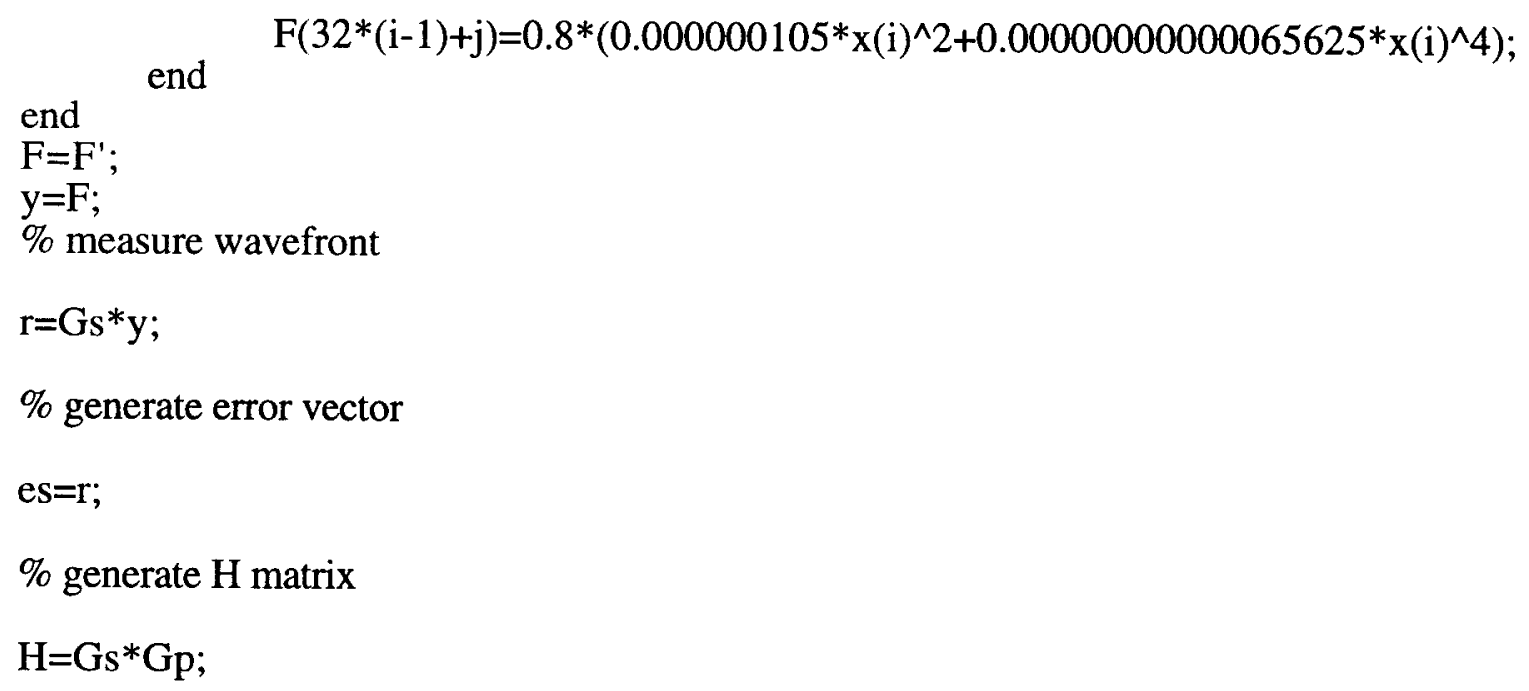

$\%$ Calculate actuator inputs coefficients using pseudo inverse and hartmann sensor $\mathrm{X}=\operatorname{inv}\left(\mathrm{H}^{\prime} * \mathrm{H}\right) * \mathrm{H}^{\prime *} \mathrm{es}$;

$\%$ use when no Hartmann sensor, only data point displacements

$\% \mathrm{X}=\operatorname{inv}\left(\mathrm{A}^{\prime *} \mathrm{~A}\right) * \mathrm{~A}^{\prime *} \mathrm{y}$;

$\%$ generate a matrix of the surface

for $i=1: 32$

for $j=1: 32$

$\mathrm{F} 1(\mathrm{i}, \mathrm{j})=\mathrm{F}(32 *(\mathrm{i}-1)+\mathrm{j})$;

end

end

$x=-200: 12.9032: 200$;

$\mathrm{y}=\mathrm{x}$;

\%display surface

$\operatorname{mesh}(\mathrm{x}, \mathrm{y}, \mathrm{F} 1)$

title('Surface to Fit')

xlabel('(mm)')

ylabel('(mm)')

zlabel('Surface Displacement (mm)')

pause

\%superposition

$\mathrm{H}=\mathrm{X}(1,1) * \mathrm{C} 01+\mathrm{X}(2,1) * \mathrm{C} 02+\mathrm{X}(3,1) * \mathrm{C} 03+\mathrm{X}(4,1) * \mathrm{C} 04+\mathrm{X}(5,1) * \mathrm{C} 05+\mathrm{X}(6,1) * \mathrm{C} 06+\mathrm{X}(7$ ,1)*C $07+\mathrm{X}(8,1) * \mathrm{C} 08+\mathrm{X}(9,1) * \mathrm{C} 09+\mathrm{X}(10,1) * \mathrm{C} 10+\mathrm{X}(11,1)^{*} \mathrm{C} 11+\mathrm{X}(12,1) * \mathrm{C} 12+\mathrm{X}(13,1$ )$^{*} \mathrm{C} 13+\mathrm{X}(14,1) * \mathrm{C} 14+\mathrm{X}(15,1) * \mathrm{C} 15+\mathrm{X}(16,1) * \mathrm{C} 16+\mathrm{X}(17,1) * \mathrm{C} 17+\mathrm{X}(18,1) * \mathrm{C} 18+\mathrm{X}(19,1$ )$^{*} \mathrm{C} 19+\mathrm{X}(20,1)^{*} \mathrm{C} 20+\mathrm{X}(21,1) * \mathrm{C} 21+\mathrm{X}(22,1) * \mathrm{C} 22+\mathrm{X}(23,1) * \mathrm{C} 23+\mathrm{X}(24,1) * \mathrm{C} 24+\mathrm{X}(25,1$ )$^{*} \mathrm{C} 25+\mathrm{X}(26,1) * \mathrm{C} 26+\mathrm{X}(27,1) * \mathrm{C} 27+\mathrm{X}(28,1) * \mathrm{C} 28+\mathrm{X}(29,1) * \mathrm{C} 29+\mathrm{X}(30,1) * \mathrm{C} 30+\mathrm{X}(31,1$ )$* \mathrm{C} 31+\mathrm{X}(32,1) * \mathrm{C} 32+\mathrm{X}(33,1) * \mathrm{C} 33+\mathrm{X}(34,1) * \mathrm{C} 34+\mathrm{X}(35,1) * \mathrm{C} 35+\mathrm{X}(36,1) * \mathrm{C} 36+\mathrm{X}(37,1$ )$* \mathrm{C} 37+\mathrm{X}(38,1) * \mathrm{C} 38+\mathrm{X}(39,1) * \mathrm{C} 39$

$\operatorname{mesh}(\mathrm{x}, \mathrm{y}, \mathrm{H})$

title('Superposition of Influence Functions')

xlabel('(mm)')

ylabel('(mm)') 


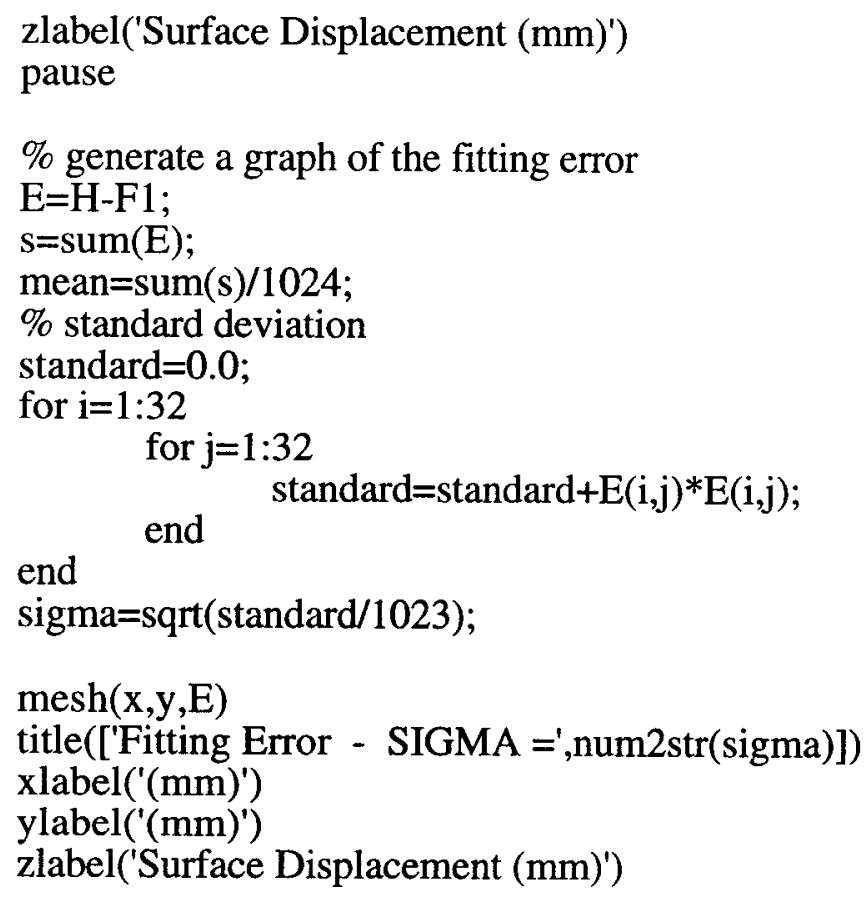




\section{APPENDIX B - C-FIELD FOR A PIEZOELECTRIC ACTUATOR.}


It is first assumed that the unloaded actuator displacement " $\delta$ " is a function of the charge " $q$ " on the capacitor. Based on experimental results, it is reasonable to assume that the actuator displacement is a linear function of the charge (over the center portion of its range)

$$
\delta=a q
$$

where " $a$ " is a scaling factor. Next, an energy function is developed which satisfies Maxwell's reciprocity. A reasonable energy function is

$$
E(x, q)=\frac{q^{2}}{2 C}+\frac{k(x-a q)^{2}}{2}
$$

where " $x "$ is the displacement of the actuator if the charge is zero and " $C$ " is the capacitance. Now derivatives are taken of the energy function with respect to the displacement " $x$ " and charge " $q$ " to develop a relationship for the force and voltage respectively, resulting in the following expressions:

$$
\begin{aligned}
& F=\frac{\partial E}{\partial x}=k(x-a q) \\
& e=\frac{\partial E}{\partial q}=\frac{q}{C}-a k(x-a q)
\end{aligned}
$$

and Maxwell's reciprocity is proven

$$
\frac{\partial F}{\partial q}=a k=\frac{\partial e}{\partial x} .
$$




\section{APPENDIX C - DERIVATION OF DYNAMIC EQUATIONS OF MOTION}


Using the Bond graphs, Figures 5.4 and 5.6 in Chapter 5, the equations of motions were developed for the single actuator and seven actuator system.

The single actuator equations of motion are derived directly from the bond graph of Figure 5.4 using existing techniques. The state variables are first identified as $q_{1}, q_{4}, q_{5}, p_{2}$. The input vector has a single entry of $S E_{7}$. From this, the state equations are written directly as

$$
\begin{gathered}
\dot{q}_{5}=\frac{1}{R}\left(S E_{7}-e_{5}\right), \\
\dot{q}_{4}=\frac{p_{2}}{I_{2}}, \\
\dot{p}_{2}=-k_{1} q_{1}-\frac{R p_{2}}{I_{2}}-e_{4} \text { and } \\
\dot{q}_{1}=\frac{p_{2}}{I_{2}} .
\end{gathered}
$$

After further examination of equations C. 1 through C.4, the four states can be simplified to three since C.2 is equal to C.4. After this simplification and substitution of the C-field representation derived in Appendix B, the equations are written as

$$
\begin{gathered}
\dot{q}_{5}=\frac{1}{R}\left[S E_{7}-\left(\frac{q_{5}}{C_{5}}-a k_{4}\left(q_{4}-a q_{5}\right)\right)\right], \\
\dot{q}_{4}=\frac{p_{2}}{I_{2}} \text { and } \\
\dot{p}_{2}=-k_{1} q_{1}-\frac{R p_{2}}{I_{2}}-k_{4}\left(q_{4}-a q_{5}\right) .
\end{gathered}
$$

Equations C.5 through C.7 have been coded in Matlab for numerical simulation. These results can be found in Appendix D. 
Next the equations of motion for the 7 actuator plate model are written. The procedure is exactly the same as with the single actuator model, the equations are written directly from the Bond graph of Figure 5.6. The same simplifications and C-fields have been used as with the single actuator development. Due to the symmetrical nature of this problem, the equations can be written in a compact form as

$$
\begin{gathered}
\dot{q}_{i}=\frac{p_{j}}{I_{j}} \\
\dot{q}_{k}=\frac{1}{R}\left[S E_{l}-\left(\frac{q_{k}}{C_{k}}-\frac{a}{C_{i}}\left(q_{i}-a q_{k}\right)\right)\right] \text { and } \\
\dot{p}_{j}=-\frac{R p_{j}}{I_{j}}-\frac{1}{C_{i}}\left(q_{i}-a q_{k}\right)-e_{i} f\left(q_{i}\right)
\end{gathered}
$$

where,

$l=(1 ; 8 ; 15 ; 22 ; 29 ; 36 ; 43)$,

$i=(4 ; 11 ; 18 ; 25 ; 32 ; 39 ; 46)$,

$j=(5 ; 12 ; 19 ; 26 ; 33 ; 40 ; 47)$,

$k=(3 ; 10 ; 17 ; 24 ; 31 ; 38 ; 45)$ and

$e_{i} f\left(q_{i}\right)=$ effort or force determined from stiffness matrix $\tilde{e}=K \tilde{q}$.

Equations C.8 through C.10, which total 21 for the seven actuator model, were coded using Matlab in Appendix E. It should be mentioned that these equations are easily expanded up to any dimension. 
APPENDIX D - SINGLE ACTUATOR MODEL CODE 
$\% \mathrm{~m}$-file to look a SISO control for single actuator (single act. for plate)

$\%$ file name: actuator $1 . m$

$\%$ written by: Scott E. Winters

$\%$ date: $3 / 98$

$\%$ parameters

$\mathrm{I} 2=0.50 ; \quad \%$ actuator/plate mass $(\mathrm{Kg})$

$\mathrm{K} 1=2000000 ; \quad \%$ plate stiffness $(\mathrm{N} / \mathrm{m})$

$\mathrm{K} 4=1000000 ; \quad \%$ actuator stiffness $(\mathrm{N} / \mathrm{m})$

$\mathrm{RM}=200.0 ; \quad \%$ mechanical resistance $(\mathrm{N} * \mathrm{~s} / \mathrm{m})$

$\mathrm{b}=1.0 ; \quad \%$ actuator gain (non dimensional)

$\mathrm{RE}=100.0 ; \quad \%$ electrical resistance (ohms)

$\mathrm{C} 5=.0000002 ; \quad \%$ actuator capacitance (Farads)

$\mathrm{KP}=0.01 ; \quad \%$ proportional gain (non dimensional)

$\mathrm{KI}=4500.00$; $\quad \%$ integral gain (non dimensional)

$\%$ A-matirx

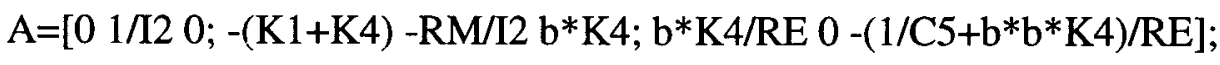

$\%$ B-matrix ( 10000000 constant is only needed to scale step response in Matlab)

$\mathrm{B}=[0 ; 0 ; 1000000 / \mathrm{RE}]$

$\%$ C-matrix

$\mathrm{C}=\left[\begin{array}{lll}1 & 0 & 0\end{array}\right]$

$\%$ D-matirx;

$\mathrm{D}=[0]$;

$\%$ develop closed loop system (proprotional and integral controller) (y/d)

[num,den] $=\operatorname{ss} 2 \mathrm{tf}(\mathrm{A}, \mathrm{B}, \mathrm{C}, \mathrm{D}, 1)$;

num $1=\operatorname{conv}([\mathrm{KP} \mathrm{KI}]$,num);

$\operatorname{den} 1=\operatorname{conv}\left(\left[\begin{array}{ll}1 & 0\end{array}\right], \operatorname{den}\right)$;

$\%$ numc $=$ den 1 ;

$\%$ denc $=$ den $1+$ num 1 ;

$\%[\mathrm{Ac}, \mathrm{Bc}, \mathrm{Cc}, \mathrm{Dc}]=\mathrm{tf} 2 \mathrm{ss}$ (numc,denc);

$\%$ develop closed loop system (PI) (y/r)

numc $=$ num1;

denc $=$ den $1+$ num 1

$[\mathrm{Ac}, \mathrm{Bc}, \mathrm{Cc}, \mathrm{Dc}]=\mathrm{tf} 2 \mathrm{ss}($ numc, denc);

\% develop of L system to match for design purposes (y/d) 


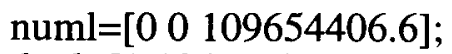

denl=[1 10471.60$]$;

$\%$ numlc=denl;

$\%$ denlc $=$ denl + numl;

$\%[\mathrm{Al}, \mathrm{Bl}, \mathrm{Cl}, \mathrm{Dl}]=\mathrm{tf} 2 \mathrm{ss}($ numlc,denlc);

$\%$ develop of $\mathrm{L}$ closed loop $(\mathrm{y} / \mathrm{r})$

$\%$ numlc=numl;

$\%$ denlc $=$ denl + numl;

$\%[\mathrm{Al}, \mathrm{Bl}, \mathrm{Cl}, \mathrm{Dl}]=\mathrm{tf} 2 \mathrm{ss}($ numlc,denlc);

\% step responses

$\% \mathrm{t}=0: .00001: 0.005$;

$\%[y l, x l]=\operatorname{step}(\mathrm{Al}, \mathrm{Bl}, \mathrm{Cl}, \mathrm{Dl}, 1, \mathrm{t})$;

$\%[\mathrm{yc}, \mathrm{xc}]=\operatorname{step}(\mathrm{Ac}, \mathrm{Bc}, \mathrm{Cc}, \mathrm{Dc}, 1, \mathrm{t})$;

$\%[\mathrm{y}, \mathrm{x}]=\operatorname{step}($ num,den,t); \% open loop response

$\%$ plotting step responses

$\%$ plot(t,y,t,yc,t,yl);

$\%$ title('step response')

\%ylabel('surface displacement (m)')

\%xlabel('time (sec.)')

$\%$ eigenvalues for closed loop systems

\%eig(A) \% open loop poles

\%eig(Ac) \% closed loop poles

\%eig(Al) \% "L" matching poles

$\%$ bode plots

$\%$ w=logspace $(2,4)$;

\%bode(A,B,C,D,1,w);

$\mathrm{T}=0: 0.0001: 0.02$;

step (A,B,C,D,1,T);

\%bode(Ac,Bc,Cc,Dc,1);

\%bode(Al,Bl,Cl,Dl,1);

\%bode(numl,denl); 


\section{APPENDIX E - MULTI-ACTUATOR MODEL CODE}


$\%$ this m-file generates $A, B, C$ and D matrices for a 7 actuator plate model

$\%$ which incorporates actuator dynamics. There are also 7 outputs for actuator

$\%$ position. Lu, Ly , $\mathrm{T}$ and $\mathrm{S}$ are also calculated. Both PI and $\mathrm{H}$-infintiy controllers

$\%$ are developed.

$\%$ written by: Scott E. Winters

$\%$ date: Mar. 18, 1998

$\%$ system parameters

$\begin{array}{ll}\mathrm{m}=.5 ; & \text { \% effective actuator/plate mass }(\mathrm{Kg}) \\ \mathrm{KA}=1000000 ; & \text { \% effective actuator stiffness }(\mathrm{N} / \mathrm{m}) \\ \mathrm{R}=200.0 ; & \% \text { damping }\left(\mathrm{N}^{*} \mathrm{~s} / \mathrm{m}\right) \\ \mathrm{g}=1.0 ; & \% \text { actuator gain (displ./charge) } \\ \mathrm{CA}=0.0000002 ; & \text { \% actuator/wire capacitance (Farads) } \\ \mathrm{RE}=100.0 ; & \text { \% electrical resistance }(\mathrm{Ohms}) \\ \mathrm{K}=2000000 ; & \text { \% plate stiffness constant }(\mathrm{N} / \mathrm{m}) \\ \mathrm{KP}=1 ; & \text { \% proportional gain } \\ \mathrm{KI}=1 ; & \text { \% integral gain } \\ \mathrm{BG}=200000 ; & \text { \% input gain to scale step response }\end{array}$

$\%$ plate stiffness matrix using $\mathrm{KA}$

$\%$ constants for plate

$\mathrm{z} 1=\mathrm{KA} /\left(6^{*} \mathrm{~K}\right)$

$\mathrm{z} 2=\mathrm{KA} /(12 * \mathrm{~K})$

$\mathrm{z} 3=(1-\mathrm{z} 1) / 20$

$\mathrm{K} 11=\mathrm{K}$;

$\mathrm{K} 12=-\mathrm{z} 1 * \mathrm{~K}$;

$\mathrm{K} 13=-\mathrm{z} 1 * \mathrm{~K}$;

$\mathrm{K} 14=-\mathrm{z} 1 * \mathrm{~K}$;

$\mathrm{K} 15=-\mathrm{z} 3^{*} \mathrm{~K}$;

$\mathrm{K} 16=-\mathrm{z} 3 * \mathrm{~K}$;

$\mathrm{K} 17=-\mathrm{z} 3{ }^{*} \mathrm{~K}$;

$\mathrm{K} 21=-\mathrm{z} 1 * \mathrm{~K}$;

$\mathrm{K} 22=\mathrm{K}$;

$\mathrm{K} 23=-\mathrm{z} 3 * \mathrm{~K}$;

$\mathrm{K} 24=-\mathrm{z} 1 * \mathrm{~K}$;

$\mathrm{K} 25=-\mathrm{z} 1 * \mathrm{~K}$;

$\mathrm{K} 26=-\mathrm{z} 3 * \mathrm{~K}$;

$\mathrm{K} 27=-\mathrm{z} 3 * \mathrm{~K}$;

$\mathrm{K} 31=-\mathrm{z} 1 * \mathrm{~K}$;

$\mathrm{K} 32=-\mathrm{z} 3^{*} \mathrm{~K}$;

$\mathrm{K} 33=\mathrm{K}$;

$\mathrm{K} 34=-\mathrm{z} 1 * \mathrm{~K}$;

$\mathrm{K} 35=-\mathrm{z} 3{ }^{*} \mathrm{~K}$;

$\mathrm{K} 36=-\mathrm{z} 1 * \mathrm{~K}$;

$\mathrm{K} 37=-\mathrm{z} 3{ }^{*} \mathrm{~K}$;

$\mathrm{K} 41=-\mathrm{z} 2 * \mathrm{~K}$

$\mathrm{K} 42=-\mathrm{z} 2 * \mathrm{~K}$; 


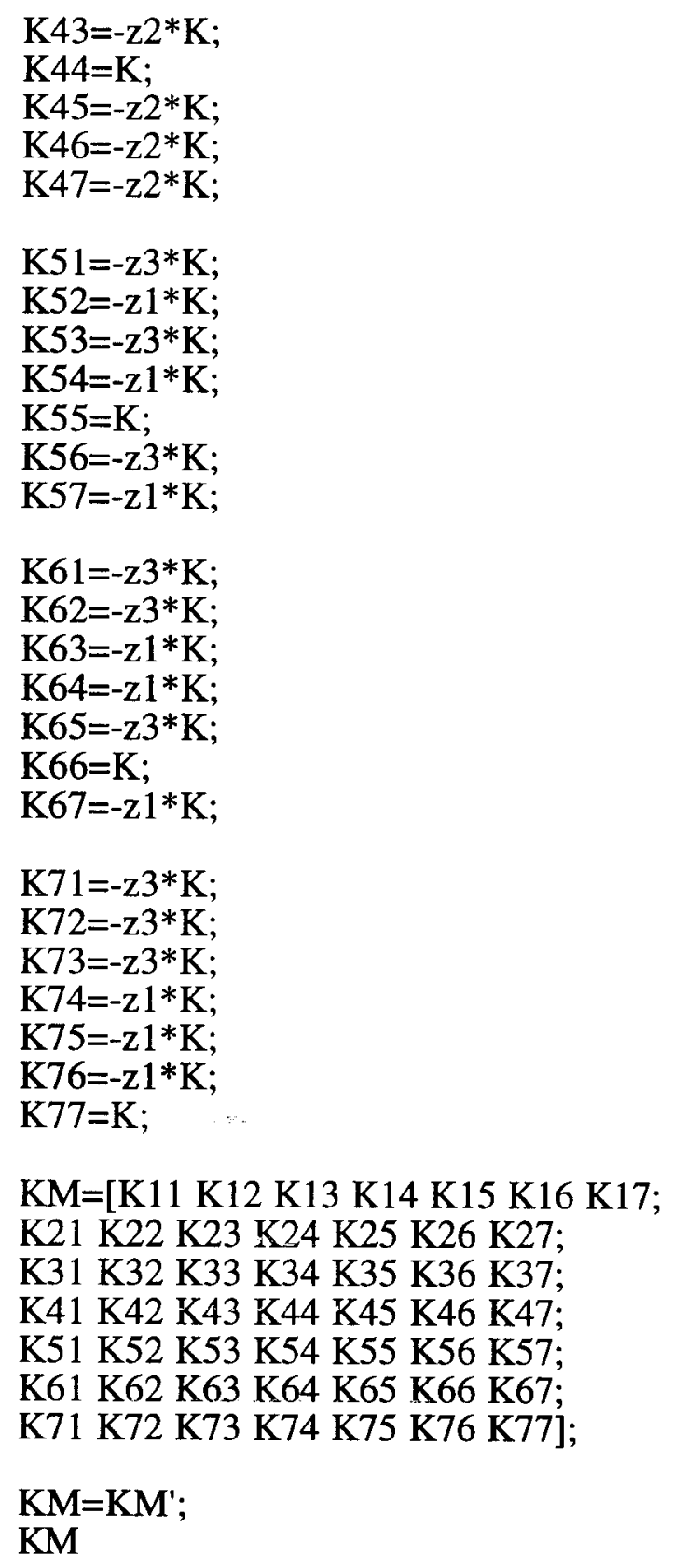

\% A-matrix indices

$$
\begin{aligned}
& \text { a0101 }=0 ; \\
& \text { a0102 }=1 / \mathrm{m} ; \\
& \text { a0103 }=0 ; \\
& \text { a0104 }=0 ; \\
& \text { a0105 }=0 ; \\
& \text { a0106 }=0 ; \\
& \text { a0107 }=0 ; \\
& \text { a0108 }=0 ; \\
& \text { a0109 }=0 ; \\
& \text { a0110 }=0 ;
\end{aligned}
$$




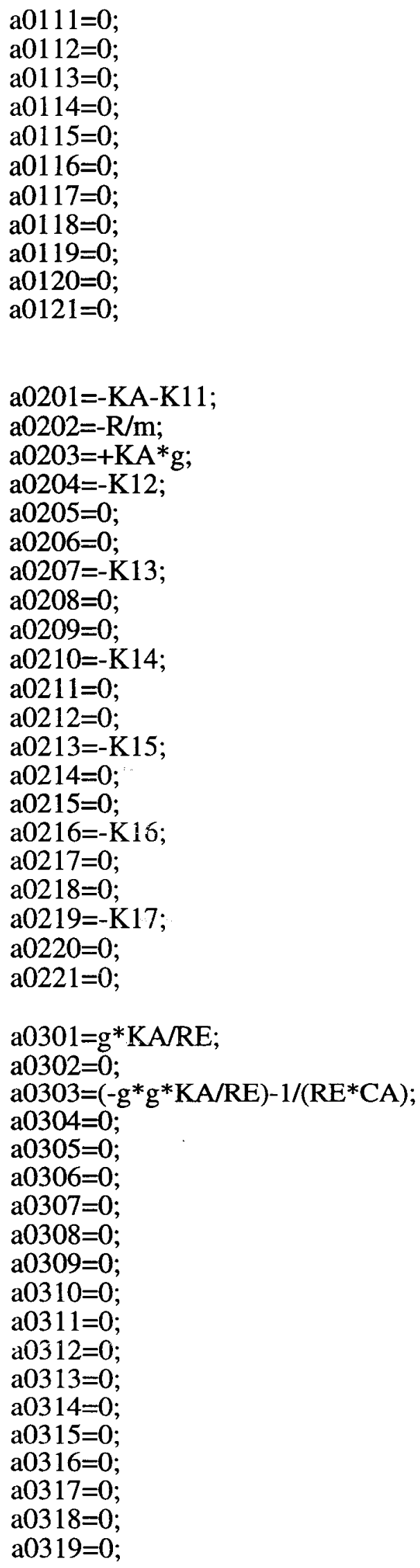


$\mathrm{a} 0320=0$;

a0321 =0;

a0401 $=0$;

a0402=0;

a0403=0;

a0404=0;

$\mathrm{a} 0405=1 / \mathrm{m}$;

a0406 $=0$;

a0407=0;

a0408=0;

a0409=0;

$\mathrm{a} 0410=0$;

a0411=0;

a0412=0;

a0413=0;

a0414=0;

a0415=0;

a0416=0;

a0417=0;

a0418=0;

a0419=0;

a0420 $=0$;

a0421 $=0$;

$\mathrm{a} 0501=-\mathrm{K} 21$;

$\mathrm{a} 0502=0$;

$\mathrm{a} 0503=0$;

a0504=-KA-K22;

$\mathrm{a} 0505=-\mathrm{R} / \mathrm{m}$;

$\mathrm{a} 0506=\mathrm{KA}^{*} \mathrm{~g}$;

a0507=-K23;

$\mathrm{a} 0508=0$;

$\mathrm{a} 0509=0$;

$\mathrm{a} 0510=-\mathrm{K} 24$;

a0511 $=0$;

$\mathrm{a} 0512=0$;

$\mathrm{a} 0513=-\mathrm{K} 25$;

a0514=0;

$\mathrm{a} 0515=0$;

a0516=-K26;

$\mathrm{a} 0517=0$;

$\mathrm{a} 0518=0$;

$\mathrm{a} 0519=-\mathrm{K} 27$;

$\mathrm{a} 0520=0$;

$\mathrm{a} 0521=0$;

$\mathrm{a} 0601=0$;

a0602 =0;

$\mathrm{a} 0603=0$;

a0604 $=\mathrm{g} * \mathrm{KA} / \mathrm{RE}$;

$\mathrm{a} 0605=0$;

a0606 $=\left(-g^{*} g * K A / R E\right)-1 /\left(R^{*}\right.$ CA $)$; 


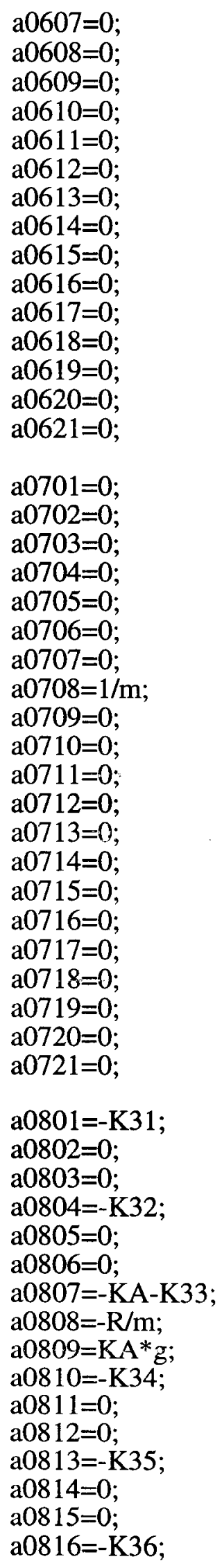




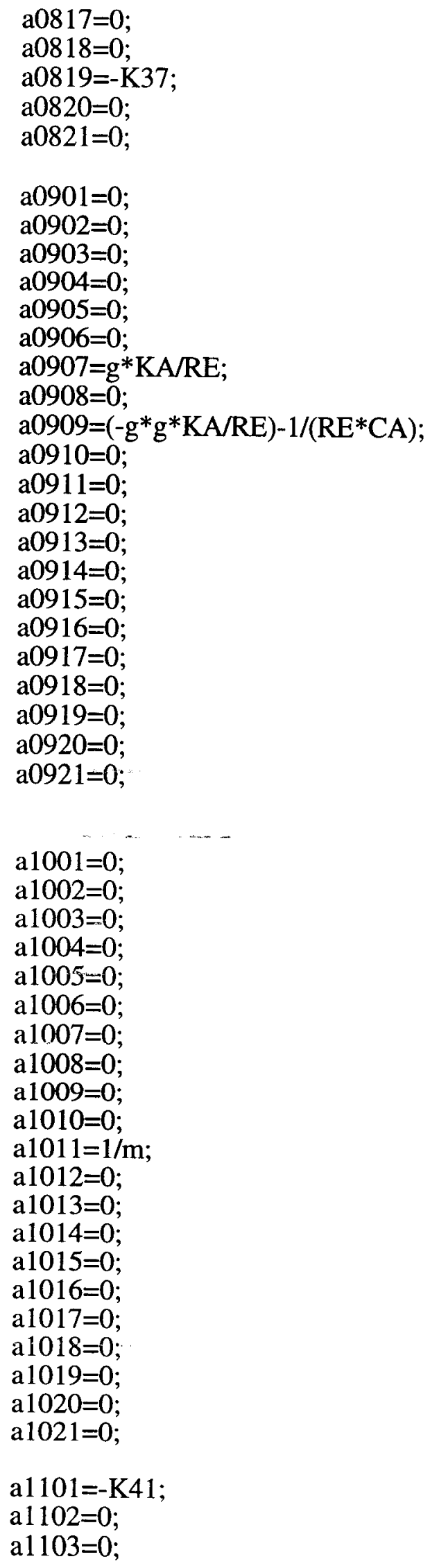




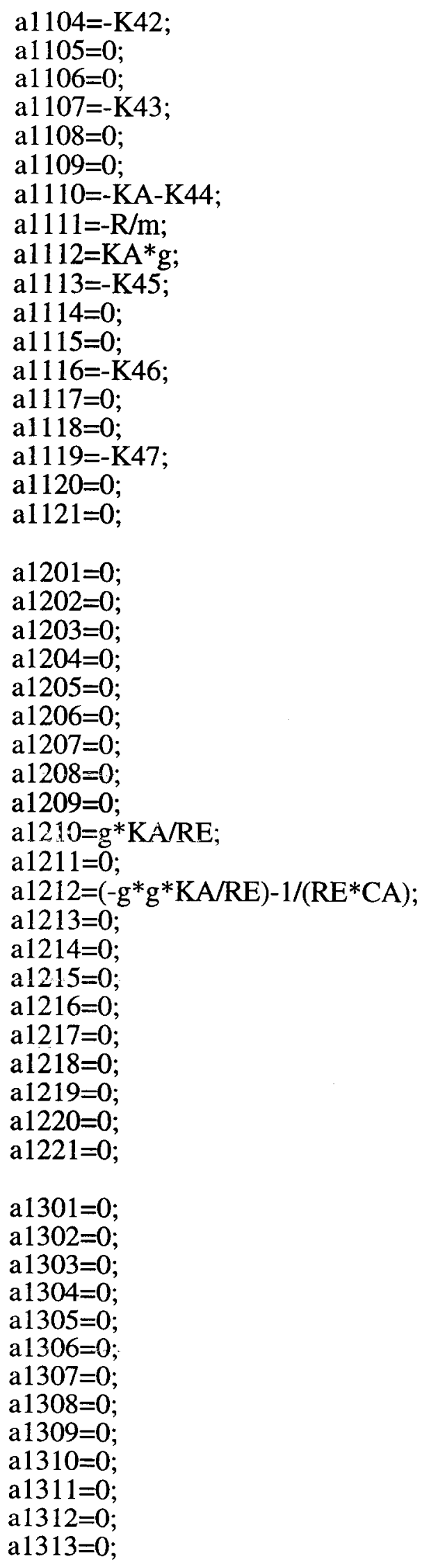




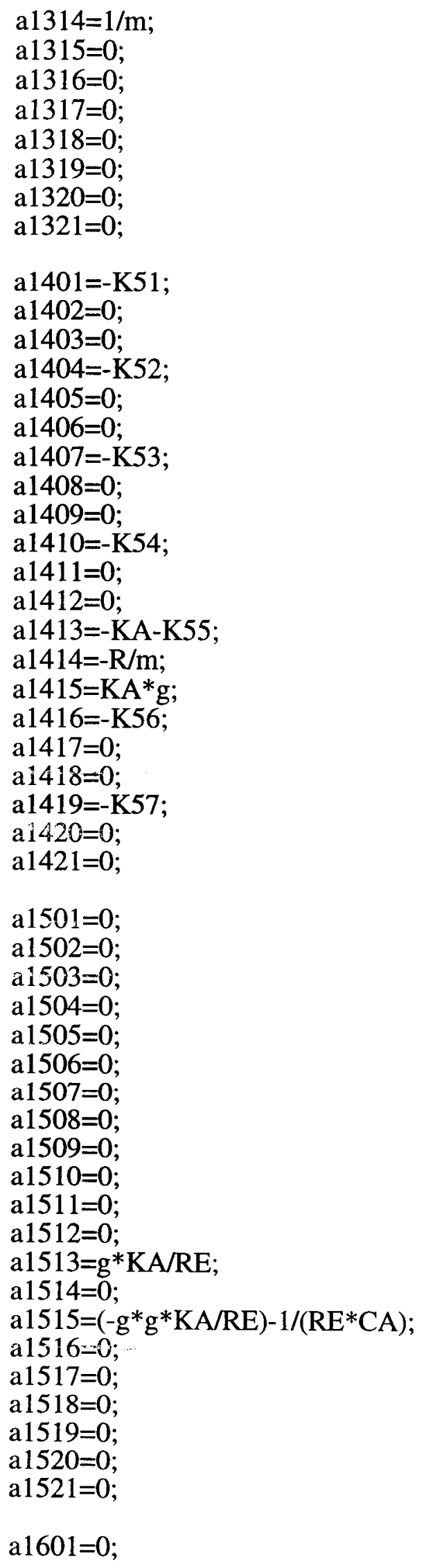




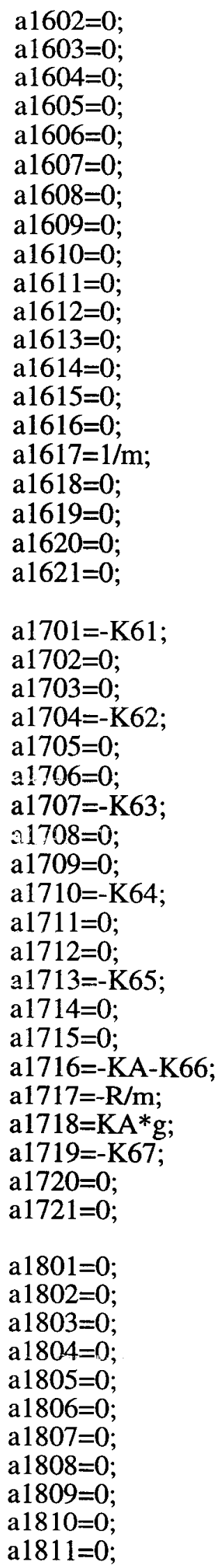




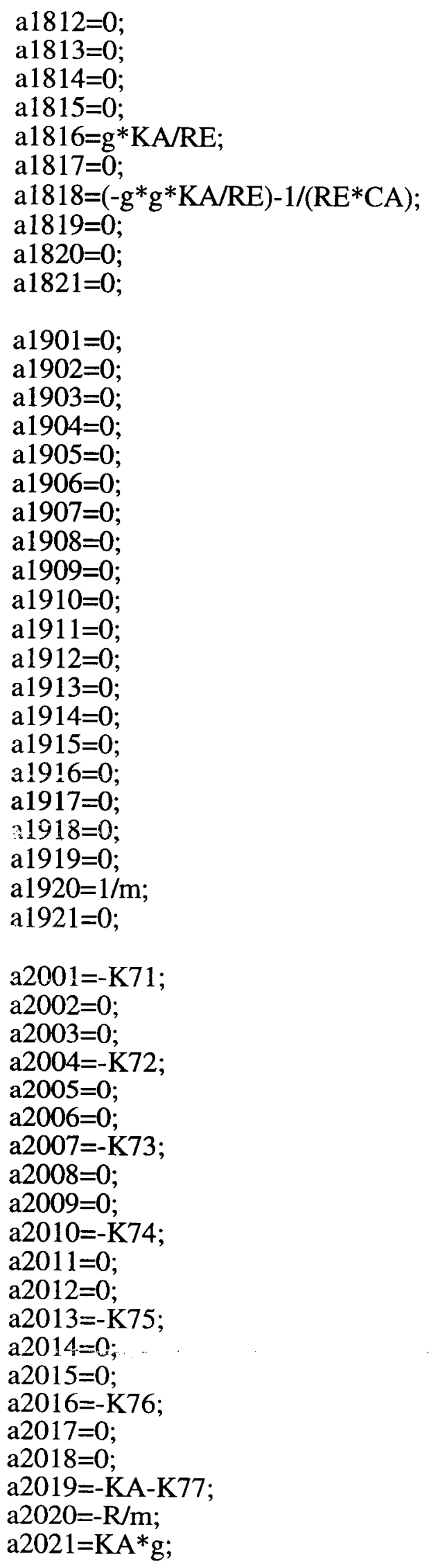




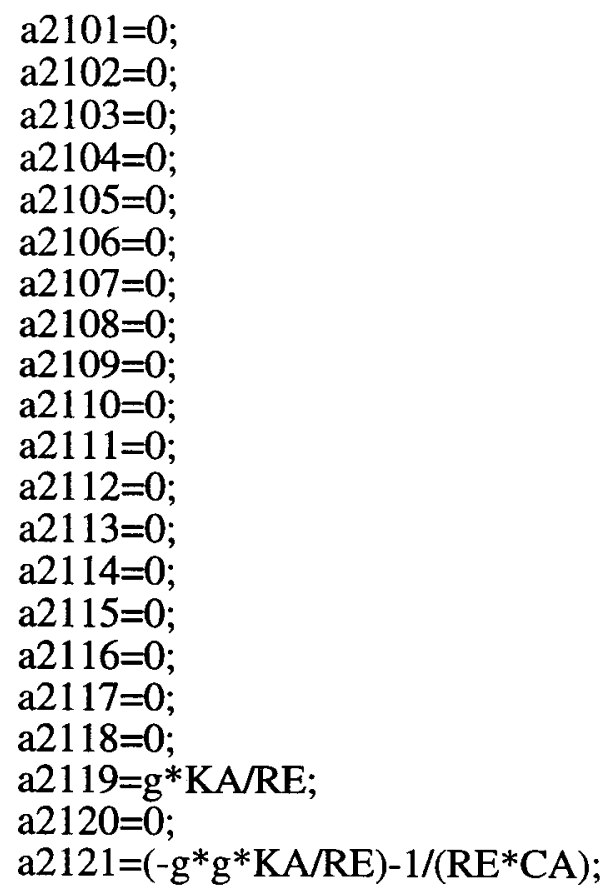

$\mathrm{A}=[\mathrm{a} 0101 \mathrm{a} 0102 \mathrm{a} 0103 \mathrm{a} 0104 \mathrm{a} 0105 \mathrm{a} 0106 \mathrm{a} 0107 \mathrm{a} 0108 \mathrm{a} 0109 \mathrm{a} 0110 \mathrm{a} 0111 \mathrm{a} 0112 \mathrm{a} 0113$ a0114 a0115 a0116 a0117 a0118 a0119 a0120 a0121; a0201 a0202 a0203 a0204 a0205 a0206 a0207 a0208 a0209 a0210 a0211 a0212 a0213 a0214 a0215 a0216 a0217 a0218 a0219 a0220 a0221; a0301 a0302 a0303 a0304 a0305 a0306 a0307 a0308 a0309 a0310 a0311 a0312 a0313 a0314 a0315 a0316 a0317 a0318 a0319 a0320 a0321; a0401 a0402 a0403 a0404 a0405 a0406 a0407 a0408 a0409 a0410 a0411 a0412 a0413 a0414 a0415 a0416 a0417 a0418 a0419 a0420 a0421; a0501 a0502 a0503 a0504 a0505 a0506 a0507 a0508 a0509 a0510 a0511 a0512 a0513 a0514 a0515 a0516 a0517 a0518 a0519 a0520 a0521; a0601 a0602 a0603 a0604 a0605 a0606 a0607 a0608 a0609 a0610 a0611 a0612 a0613 a0614 a0615 a0616 a0617 a0618 a0619 a0620 a0621;

a0701 a0702 a0703 a0704 a0705 a0706 a0707 a0708 a0709 a0710 a0711 a0712 a0713 a0714 a0715 a0716 a0717 a0718 a0719 a0720 a0721; a0801 a0802 a0803 a0804 a0805 a0806 a0807 a0808 a0809 a0810 a0811 a0812 a0813 a0814 a0815 a0816 a0817 a0818 a0819 a0820 a0821; a0901 a0902 a0903 a0904 a0905 a0906 a0907 a0908 a0909 a0910 a0911 a0912 a0913 a0914 a0915 a0916 a0917 a0918 a0919 a0920 a0921; a1001 a1002 a1003 a1004 a1005 a1006 a1007 a1008 a1009 a1010 a1011 a1012 a1013 a1014 a1015 a1016 a1017 a1018 a1019 a1020 a1021; a1 101 a1 102 a1103 a1104 a1 105 a1 106 a1 107 a1 108 a1109 a1110 a1111 a1112 a1113 a1114 a1115 a1116 a1117 a1118 a1119 a1120 a1121; a1201 a1202 a1203 a1204 a1205 a1206 a1207 a1208 a1209 a1210 a1211 a1212 a1213 a1214 a1215 a1216 a1217 a1218 a1219 a1220 a1221; a1301 a1302 a1303 a1304 a1305 a1306 a1307 a1308 a1309 a1310 a1311 a1312 a1313 a1314 a1315 a1316 a1317 a1318 a1319 a1320 a1321; a1401 a1402 a1403 a1404 a1405 a1406 a1407 a1408 a1409 a1410 a1411 a1412 a1413 a1414 a1415 a1416 a1417 a1418 a1419 a1420 a1421; a1501 a1502 a1503 a1504 a1505 a1506 a1507 a1508 a1509 a1510 a1511 a1512 a1513 a1514 a1515 a1516 a1517 a1518 a1519 a1520 a1521; 
a1601 a1602 a1603 a1604 a1605 a1606 a1607 a1608 a1609 a1610 a1611 a1612 a1613 a1614 a1615 a1616 a1617 a1618 a1619 a1620 a1621;

a1701 a1702 a1703 a1704 a1705 a1706 a1707 a1708 a1709 a1710 a1711 a1712 a1713 a1714 a1715 a1716 a1717 a1718 a1719 a1720 a1721;

a1801 a1802 a1803 a1804 a1805 a1806 a1807 a1808 a1809 a1810 a1811 a1812 a1813 a1814 a1815 a1816 a1817 a1818 a1819 a1820 a1821;

a1901 a1902 a1903 a1904 a1905 a1906 a1907 a1908 a1909 a1910 a1911 a1912 a1913 a1914 a1915 a1916 a1917 a1918 a1919 a1920 a1921;

a2001 a2002 a2003 a2004 a2005 a2006 a2007 a2008 a2009 a2010 a2011 a2012 a2013 a2014 a2015 a2016 a2017 a2018 a2019 a2020 a2021;

a2101 a2102 a2103 a2104 a2105 a2106 a2107 a2108 a2109 a2110 a2111 a2112 a2113 a2114 a2115 a2116 a2117 a2118 a2119 a2120 a2121];

\% B-matrix

b0101=0;

b0102=0;

b0103=0;

b0104=0;

b0105=0;

b0106 =0;

b0107=0;

b0201 $=0$;

b0202=0;

b0203=0;

b0204=0;

b0205=0;

b0206=0;

b0207=0;

$\mathrm{b} 0301=\mathrm{BG} / \mathrm{RE}$;

b0302 $=0$;

b0303=0;

b0304=0;

b0305=0;

b0306 $=0$;

b0307=0;

b0401=0;

b0402=0;

b0403=0;

b0404=0;

b0405=0;

b0406=0;

b0407=0;

b0501 $=0$;

b0502=0;

b0503=0;

b0504=0;

b0505=0;

b0506 $=0$;

b0507=0; 


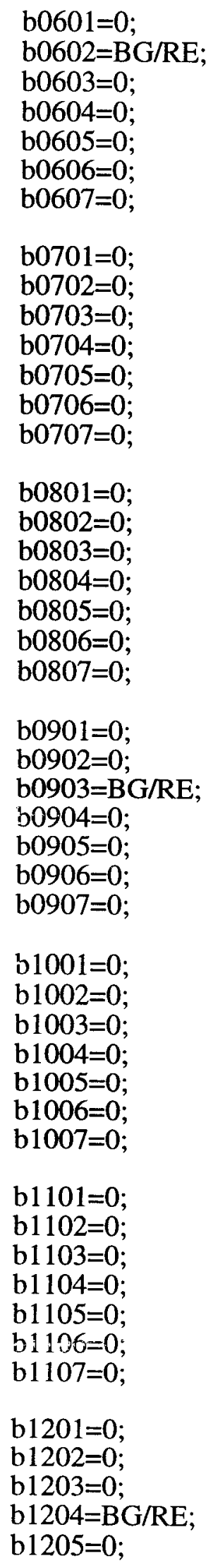

b1 101=0;

b1 102 =0;

b1 103 =0;

b1 104=0;

b1 105=0;

b1106=0;

b1 107=0;

b1201=0;

b1202=0;

b1203 $=0$;

b $1204=\mathrm{BG} / \mathrm{RE}$;

b1205=0; 


$$
\begin{aligned}
& \text { b1206=0; } \\
& \text { b1207=0; } \\
& \text { b1301=0; } \\
& \text { b1302=0; } \\
& \text { b1303=0; } \\
& \text { b1304=0; } \\
& \text { b1305=0; } \\
& \text { b1306 }=0 \text {; } \\
& \text { b1307=0; } \\
& \text { b1401 }=0 \text {; } \\
& \text { b1402=0; } \\
& \text { b1403=0; } \\
& \text { b1404=0; } \\
& \text { b1405=0; } \\
& \text { b1406=0; } \\
& \text { b1407=0; } \\
& \text { b1501=0; } \\
& \text { b1502=0; } \\
& \text { b1503=0; } \\
& \text { b1504=0; } \\
& \text { b1505=BG/RE; } \\
& \text { b1506 }=0 \text {; } \\
& \text { b1507=0; } \\
& \text { b1601=0; } \\
& \text { b1602=0; } \\
& \text { b1603=0; } \\
& \text { b1604=0; } \\
& \text { b1605=0; } \\
& \text { b1606=0; } \\
& \text { b1607=0; } \\
& \text { b1701 }=0 \text {; } \\
& \text { b1702=0; } \\
& \text { b1703=0; } \\
& \text { b1704=0; } \\
& \text { b1705=0; } \\
& \text { b1706=0; } \\
& \text { b } 1707=0 \text {; } \\
& \text { b1801=0; } \\
& \text { b1802=0; } \\
& \text { b1 } 1803=0 \text {; } \\
& \text { b1804=0; } \\
& \text { b } 1805=0 \text {; } \\
& \text { b1806=BG/RE; } \\
& \text { b1807=0; } \\
& \text { b1901=0; } \\
& \text { b1902=0; } \\
& \text { b1903=0; }
\end{aligned}
$$




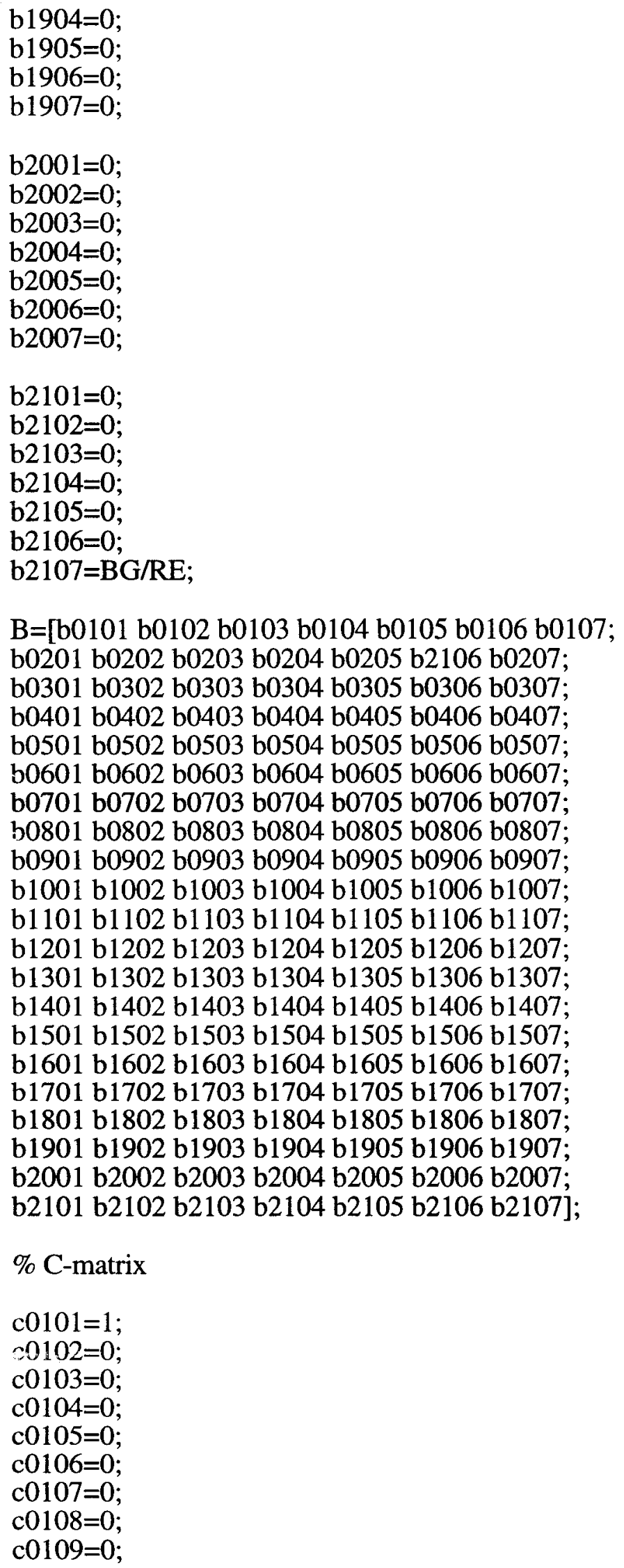




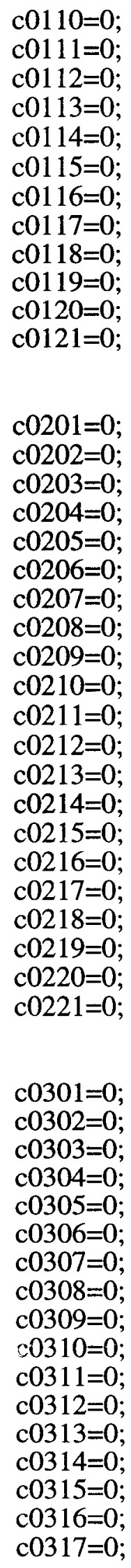

$\mathrm{c} 0301=0$; c0302 $=0$; c0303 $=0$; c0304 $=0$; c0305 $=0$; c0306 $=0$; c0307 $=0$; c0308 $=0$; c0309 $=0$; с0310 $=0$; c0311=0; c0312=0; c0313=0; c0314=0; c0315=0; c0316=0; c0317=0; 


$$
\begin{aligned}
& \text { c0318 }=0 \text {; } \\
& \text { c0319=0; } \\
& \mathrm{c} 0320=0 \text {; } \\
& \text { c0321 }=0 \text {; } \\
& \mathrm{c} 0401=0 \text {; } \\
& \text { c0402=0; } \\
& \mathrm{c} 0403=0 \text {; } \\
& \text { c0404=1; } \\
& \mathrm{c} 0405=0 \text {; } \\
& \text { c0406 }=0 \text {; } \\
& \text { c0407 }=0 \text {; } \\
& \mathrm{c} 0408=0 \text {; } \\
& \text { c0409=0; } \\
& \text { c0410 }=0 \text {; } \\
& \text { c0411 }=0 \text {; } \\
& \text { c0412=0; } \\
& \text { c0413=0; } \\
& \text { c0414=0; } \\
& \text { c0415=0; } \\
& \text { c0416=0; } \\
& \text { c0417 }=0 \text {; } \\
& \mathrm{c} 0418=0 \text {; } \\
& \text { c0419 }=0 \text {; } \\
& \mathrm{c} 0420=0 \text {; } \\
& \text { c0421 =0; } \\
& \mathrm{c} 0501=0 \text {; } \\
& \mathrm{c} 0502=0 \text {; } \\
& \text { c0503 }=0 \text {; } \\
& \text { c0504=0; } \\
& \mathrm{c} 0505=0 \text {; } \\
& \text { c0506 }=0 \text {; } \\
& \mathrm{c} 0507=0 \text {; } \\
& \mathrm{c} 0508=0 \text {; } \\
& \mathrm{c} 0509=0 \text {; } \\
& \mathrm{c} 0510=0 \text {; } \\
& \operatorname{co5} 11=0 \text {; } \\
& \text { c0512=0; } \\
& \mathrm{c} 0513=0 \text {; } \\
& \text { c0514=0; } \\
& \mathrm{c} 0515=0 \text {; } \\
& \text { c0516 }=0 \text {; } \\
& \mathrm{c} 0517=0 \text {; } \\
& \operatorname{co5} 18=0 \text {; } \\
& \text { c0519=0; } \\
& \text { c0520 }=0 \text {; } \\
& \text { c0521 }=0 \text {; } \\
& \mathrm{c} 0601=0 \text {; } \\
& \text { c0602 }=0 \text {; } \\
& \mathrm{c} 0603=0 \text {; } \\
& \text { c0604 =0; } \\
& \text { c0605 }=0 \text {; }
\end{aligned}
$$




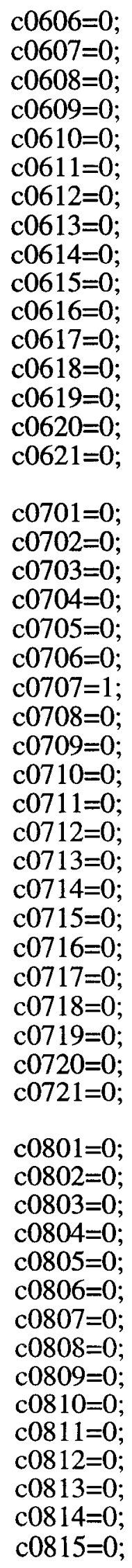




$$
\begin{aligned}
& \text { c0816=0; } \\
& \mathrm{c} 0817=0 \text {; } \\
& \text { c0818=0; } \\
& \mathrm{c} 0819=0 \text {; } \\
& \mathrm{c} 0820=0 \text {; } \\
& \text { c0821 =0; } \\
& \mathrm{c} 0901=0 \text {; } \\
& \text { c0902 }=0 \text {; } \\
& \text { c0903=0; } \\
& \text { c0904=0; } \\
& \text { c0905 =0; } \\
& \text { c0906 }=0 \text {; } \\
& \text { c0907 }=0 \text {; } \\
& \mathrm{c} 0908=0 \text {; } \\
& \mathrm{c} 0909=0 \text {; } \\
& \mathrm{c} 0910=0 \text {; } \\
& \text { c0911=0; } \\
& \text { c0912 }=0 \text {; } \\
& \text { c0913=0; } \\
& \text { c0914=0; } \\
& \text { c0915=0; } \\
& \text { c0916 }=0 \text {; } \\
& \mathrm{c} 0917=0 \text {; } \\
& \text { c0918=0; } \\
& \text { c0919=0; } \\
& \mathrm{c} 0920=0 \text {; } \\
& \mathrm{c} 0921=0 \text {; } \\
& \mathrm{c} 1001=0 \text {; } \\
& \mathrm{c} 1002=0 \text {; } \\
& \mathrm{c} 1003=0 \text {; } \\
& \text { c } 1004=0 \text {; } \\
& \text { c } 1005=0 \text {; } \\
& \text { c } 1006=0 \text {; } \\
& \mathrm{c} 1007=0 \text {; } \\
& \text { c } 1008=0 \text {; } \\
& \text { c } 1009=0 \text {; } \\
& \text { c1010=1; } \\
& \text { c1011 =0; } \\
& \text { c1012=0; } \\
& \text { c 1013=0; } \\
& \text { c1014=0; } \\
& \text { c } 1015=0 \text {; } \\
& \text { c1016 }=0 \text {; } \\
& \text { c } 1017=0 \text {; } \\
& \text { c1018=0; } \\
& \text { c1019=0; } \\
& \text { c1020 }=0 \text {; } \\
& \text { c } 1021=0 \text {; } \\
& \text { c1101 }=0 \text {; } \\
& \text { c1102 }=0 \text {; } \\
& \text { c1103=0; }
\end{aligned}
$$


c1104=0;

c1 105 =0;

c1106 $=0$;

c1107=0;

c1 108 $=0$;

c1 109=0;

c1110 $=0$;

c1111=0;

c1112=0;

c1113=0;

c1114=0;

c1115=0;

c1116=0;

c1117=0;

c1118=0;

c1119=0;

c1120 $=0$;

c1121=0;

$\mathrm{c} 1201=0$;

c1202 $=0$;

c1203 $=0$;

c1204=0;

c1205 =0;

c1206 =0;

c1207=0;

c1208=0;

c1209 $=0$;

c1210 $=0$;

c1211=0;

c1212=0;

c1213=0;

c1214=0;

c1215 $=0$;

c1216 =0;

c1217=0;

c1218=0;

c1219=0;

c1220 $=0$;

c1221 =0;

c1301=0;

c1302 $=0$;

c1303 $=0$;

c1304 =0;

c1305 =0;

c1306 $=0$;

c $1307=0$;

c $1308=0$;

c $1309=0$;

c $1310=0$;

c1311=0;

c1312 =0;

c1313=1; 


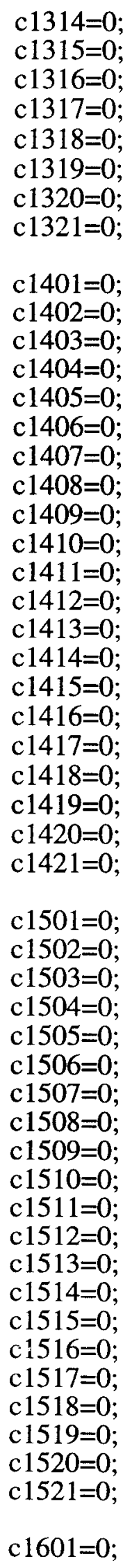



c1602 $=0$;
c1603 $=0$;
c1604=0;
c1605 $=0$;
c1606 $=0$;
$\mathrm{c} 1607=0$;
c1608=0;
c1609 $=0$;
c1610 $=0$;
$\mathrm{c} 1611=0$;
c1612=0;
c1613=0;
c1614=0;
c1615=0;
c1616=1;
c1617=0;
c1618=0;
c1619=0;
$\mathrm{c} 1620=0$;
c1621=0;
$\mathrm{c} 1701=0$;
c1702 $=0$;
c1703 $=0$;
c1704=0;
c $1705=0$;
c $1706=0$;
c $1707=0$;
c $1708=0$;
c $1709=0$;
c1710 $=0$;
c1711=0;
c1712=0;
$\mathrm{c} 1713=0$;
c1714=0;
c $1715=0$;
c1716=0;
c1717=0;
c1718=0;
c1719=0;
c1720 $=0$;
c1721 $=0$;
$\mathrm{c} 1801=0$;
c1802=0;
c $1803=0$;
c 1804=0;
c $1805=0$;
c $1806=0$;
c $1807=0$;
c $1808=0$;
c $1809=0$;
c $1810=0$;
$\mathrm{c} 1811=0$; 


$$
\begin{aligned}
& \text { c1812=0; } \\
& \text { c1813=0; } \\
& \text { c1814=0; } \\
& \text { c1815=0; } \\
& \text { c1816=0; } \\
& \text { c1817=0; } \\
& \text { c1818=0; } \\
& \text { c1819=0; } \\
& \text { c1820=0; } \\
& \mathrm{c} 1821=0 \text {; } \\
& \text { c1901=0; } \\
& \text { c1902=0; } \\
& \mathrm{c} 1903=0 \text {; } \\
& \text { c1904=0; } \\
& \text { c1905=0; } \\
& \text { c1906=0; } \\
& \text { c1907=0; } \\
& \text { c1908=0; } \\
& \text { c1909=0; } \\
& \mathrm{c} 1910=0 \text {; } \\
& \mathrm{c} 1911=0 \text {; } \\
& \text { c1912=0; } \\
& \mathrm{c} 1913=0 \text {; } \\
& \text { c1914=0; } \\
& \text { c1915=0; } \\
& \text { c1916=0; } \\
& \text { c1917=0; } \\
& \text { c1918=0; } \\
& \text { c1919=1; } \\
& \text { c } 1920=0 \text {; } \\
& \text { c1921=0; } \\
& \text { c2001 }=0 \text {; } \\
& \text { c2002 }=0 \text {; } \\
& \text { c2003 }=0 \text {; } \\
& \text { c2004=0; } \\
& \text { c2005 =0; } \\
& \text { c2006 }=0 \text {; } \\
& \text { c2007=0; } \\
& \text { c2008=0; } \\
& \text { c2009 }=0 \text {; } \\
& \text { c2010 }=0 \text {; } \\
& \text { c2011=0; } \\
& \text { c2012=0; } \\
& \text { c2013=0; } \\
& \text { c2014=0; } \\
& \text { c2015=0; } \\
& \text { c2016=0; } \\
& \text { c2017 }=0 \text {; } \\
& \text { c2018=0; } \\
& \text { c2019=0; } \\
& \text { c } 2020=0 \text {; } \\
& \text { c2021 =0; }
\end{aligned}
$$


c2101 $=0 ;$
c2102 $=0 ;$
c2103 $=0 ;$
c2104 $=0 ;$
c2105 $=0 ;$
c2106 $=0 ;$
c2107 $=0 ;$
c2108 $=0 ;$
c2109=0;
c2110 $=0 ;$
c2111 $=0 ;$
c2112 $=0 ;$
c2113 $=0 ;$
c2114 $=0 ;$
c2115 $=0 ;$
c2116 $=0 ;$
c2117=0;
c2118 $=0 ;$
c2119=0;
c2120 $=0 ;$
c2121 $=0 ;$

$\mathrm{C}=[\mathrm{c} 0101 \mathrm{c0102} \mathrm{c0103} \mathrm{c0104} \mathrm{c0105} \mathrm{c0106} \mathrm{c0107} \mathrm{c0108} \mathrm{c0109} \mathrm{c0110} \mathrm{c0111} \mathrm{c0112} \mathrm{c0113}$ $\mathrm{c} 0114 \mathrm{c} 0115 \mathrm{c} 0116 \mathrm{c} 0117 \mathrm{c} 0118 \mathrm{c} 0119 \mathrm{c} 0120 \mathrm{c} 0121$;

c0401 c0402 c0403 c0404 c0405 c0406 c0407 c0408 c0409 c0410 c0411 c0412 c0413 c0414 c0415 c0416 c0417 c0418 c0419 c0420 c0421;

c0701 c0702 c0703 c0704 c0705 c0706 c0707 c0708 c0709 c0710 c0711 c0712 c0713 c0714 c0715 c0716 c0717 c0718 c0719 c0720 c0721;

c1001 c1002 c1003 c1004 c1005 c1006 c1007 c1008 c1009 c1010 c1011 c1012 c1013 c1014 c1015 c1016 c1017 c1018 c1019 c1020 c1021;

c1301 c1302 c1303 c1304 c1305 c1306 c1307 c1308 c1309 c1310 c1311 c1312 c1313 c1314 c1315 c1316 c1317 c1318 c1319 c1320 c1321;

c1601 c1602 c1603 c1604 c1605 c1606 c1607 c1608 c1609 c1610 c1611 c1612 c1613 c1614 c1615 c1616 c1617 c1618 c1619 c1620 c1621;

c1901 c1902 c1903 c1904 c1905 c1906 c1907 c1908 c1909 c1910 c1911 c1912 c1913 c1914 c1915 c1916 c1917 c1918 c1919 c1920 c1921];

\% D-matrix

$\mathrm{D}=\left[\begin{array}{llllllll}0 & 0 & 0 & 0 & 0 & 0 & 0\end{array}\right.$

0000000 ;

0000000 ;

0000000 ;

0000000 ;

0000000 ;

$\left.\begin{array}{llllllll}0 & 0 & 0 & 0 & 0 & 0 & 0\end{array}\right]$

$\%$ eigenvalues for open loop system

$\%$ eig(A)

$\%$ Step response for open loop system 


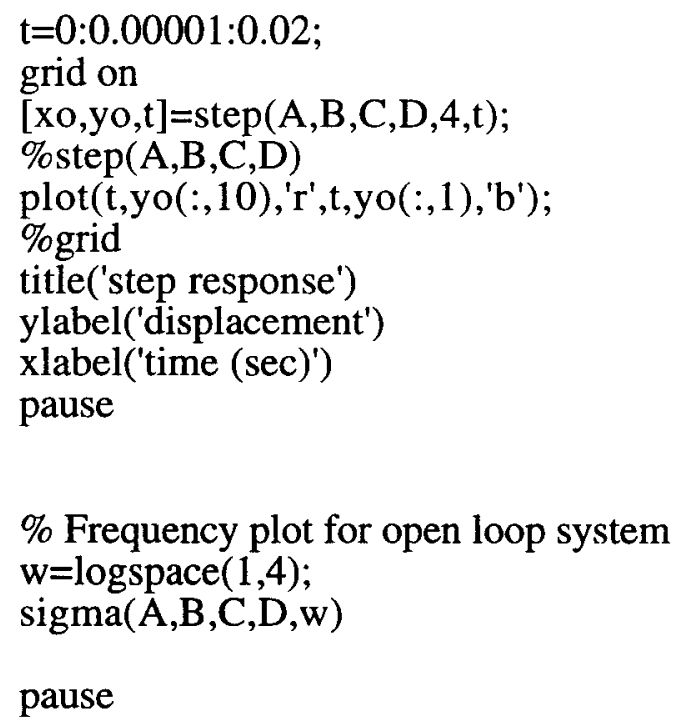




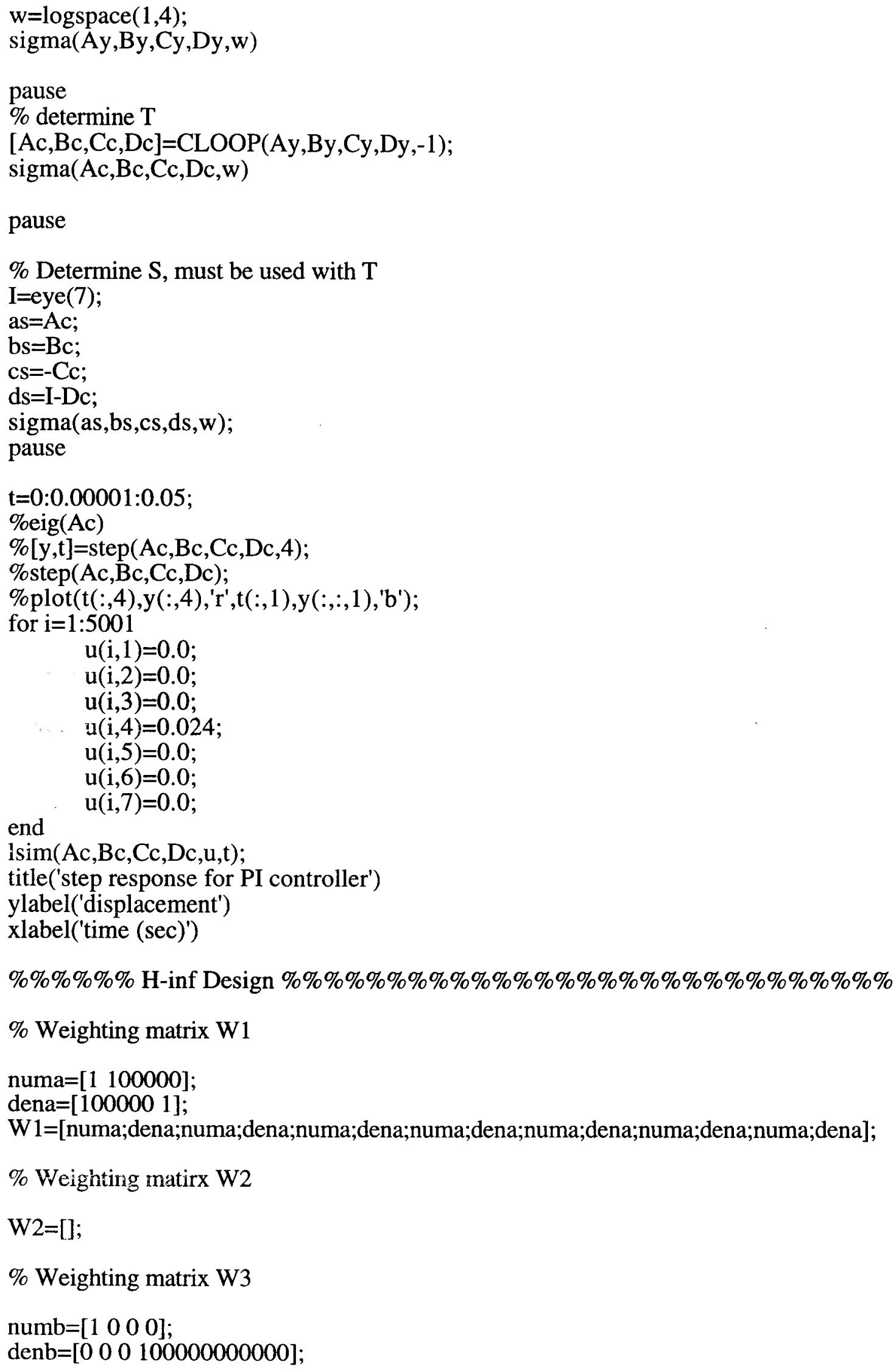


W3=[numb;denb;numb;denb;numb;denb;numb;denb;numb;denb;numb;denb;numb;denb];

\% Create the data structure

$\operatorname{ssg}=\operatorname{mksys}(\mathrm{A}, \mathrm{B}, \mathrm{C}, \mathrm{D})$;

$\%$ Create the data structure for the augmented system

ssga=augtf(ssg,W1,W2,W3);

\% Compute a controller $\mathrm{K}$ to minimize $|\mathrm{T}(\mathrm{zu})|<1$

$[$ ssk,sscl] $=$ hinf $($ ssga);

\% Determine the state realization of controller $\mathrm{K}$

[ak,bk,ck,dk]=branch(ssk);

$\%$ Determine singular value plot of |Tzwl

\%sigma(sscl);

\% Determine $\mathrm{L}=\mathrm{GK}$ from series command

[al,bl,cl,dl] =series(ak,bk,ck,dk,A,B,C,D);

$\mathrm{w}=\operatorname{logspace}(0,4)$;

sigma(al,bl,cl,dl,w);

pause

\% Determine $\mathrm{T}$

$[a t, b t, c t, d t]=C L O O P(a l, b l, c l, d l,-1)$;

sigma(at,bt,ct,dt,w);

pause

\% Determine S, must be used with T I=eye(7);

as $=$ at;

$\mathrm{bs}=\mathrm{bt}$;

$\mathrm{cs}=$-ct;

$\mathrm{ds}=\mathrm{I}-\mathrm{dt}$;

$\operatorname{sigma}($ as,bs,cs,ds,w);

pause

\% Step Response

$\% \mathrm{t}=0: 0.00001: 0.02$;

$\%[\mathrm{x}, \mathrm{y}, \mathrm{t}]=\operatorname{step}(\mathrm{at}, \mathrm{bt}, \mathrm{ct}, \mathrm{dt}, 4, \mathrm{t})$;

\%step(at,bt,ct,dt); 


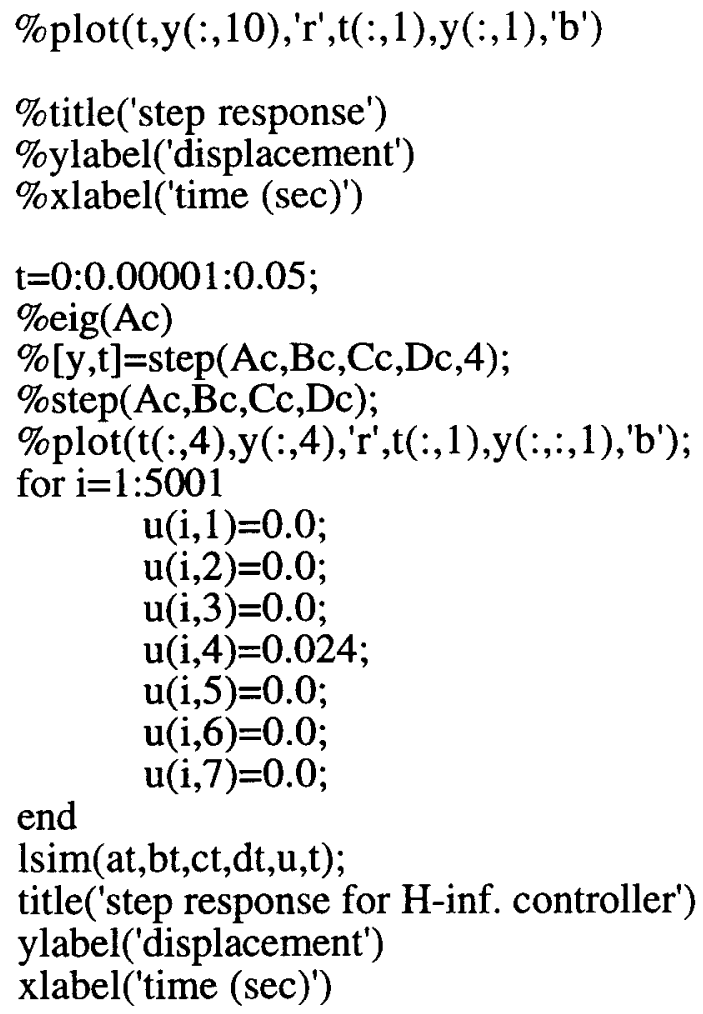

